\title{
Poisson, Bayes, Futebol e DeFinetti
}

\section{Marcelo Leme de Arruda}

\author{
DISSERTAÇÃO APRESENTADA \\ $\mathrm{AO}$ \\ INSTITUTO DE MATEMÁTICA E ESTATÍSTICA \\ DA \\ UNIVERSIDADE DE SÃO PAULO \\ PARA \\ OBTENÇÃO DO TÍTULO \\ DE \\ MESTRE EM ESTATÍSTICA
}

Área de Concentração: Estatística

Orientador: Prof. Dr. Sergio Wechsler

Durante o desenvolvimento deste trabalho o autor teve o apoio financeiro da FAPESP

São Paulo, 31 de março de 2000 


\section{Poisson, Bayes, Futebol e DeFinetti}

Este exemplar corresponde à redação final da tese defendida por Marcelo Leme de Arruda e aprovada pela comissão julgadora

São Paulo, 28 de abril de 2000

Banca examinadora:

- Prof. Dr. Sergio Wechsler (orientador) (IME-USP)

- Prof. Dr. Adilson Simonis (IME-USP)

- Prof. Dr. Gauss Moutinho Cordeiro (UFBA) 
"Está sendo atingido um público leigo muito grande. Além de considerar-se o papel do futebol no lazer da sociedade brasileira, deve ser ressaltada a contribuição educativa que se alcança, uma vez que cada vez mais o cidadão necessita de uma compreensão mínima da linguagem e postura probabilísticas, essencial em todas as realizações científicas que o atingem e o beneficiam. Essa necessidade está presente na leitura de previsões meteorológicas, diagnósticos clínicos, previsões econômicas, previsões eleitorais e tantas outras." 


\title{
RESUMO
}

Nesta dissertação é abordado o problema de previsões probabilísticas para eventos tricotômicos, além da questão de comparação de qualidade das previsões através das curvas de calibração e da Medida de DeFinetti. É feita uma aplicação para previsões de resultados de futebol.

\begin{abstract}
This dissertation deals with the problem of probabilistic previsions for tricotomic events, in addiction with the question of comparison of quality of the previsions, by using calibration curves and the DeFinetti Measure. An application is developed for previsions of soccer games results.
\end{abstract}




\section{ÍNDICE}

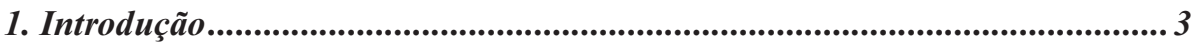

2. As Distribuiçães de Poisson Bivariadas.......................................................... 4

2.1. A Classe Geral de Distribuições de Poisson Bivariadas ....................... 4

2.2. A Classe "de Holgate"........................................................................ 4

2.2.1. Construção da Densidade ....................................................... 5

2.2.2. Formas de Estimação dos Parâmetros com amostras IID......... 5

2.2.3. A Identificabilidade dos Parâmetros........................................... 6

2.2.4. Comentários sobre o Parâmetro Covariância........................... 7

2.3. Outras Classes Poisson Bivariadas ..................................................... 8

2.3.1. A Classe Poisson Bivariada "de Brooks" ................................ 8

2.3.2. Uma Classe Poisson Bivariada com Covariância Nula e

Dependência ........................................................................ 9

2.4. Caracterização da Classe Poisson Bivariada Geral ............................. 9

2.5. As Classes Poisson Bivariadas e a Previsão de Resultados em

Jogos de Futebol. 10

3. Métodos de Estimação de Parâmetros para Previsão Probabilística de

Resultados de Jogos de Futebol ......................................................................... 12

3.1. Esclarecimentos Iniciais.................................................................... 12

3.2. A "Transitividade" nos Métodos ..................................................... 13

3.3. Família de Métodos SD (Soma e Diferença) ........................................ 13

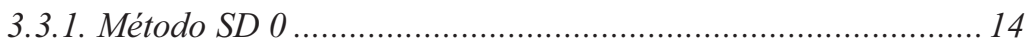

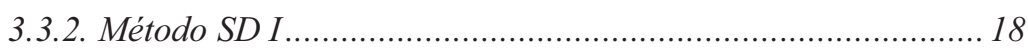

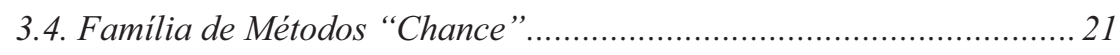

3.4.1. Método "Chance" I............................................................. 21

3.4.2. Método "Chance” II .................................................................. 24

3.5. Família de Métodos Implícitos ou Diretos …………...........................2 27

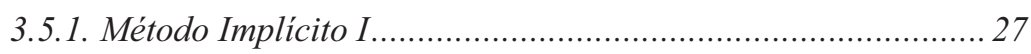

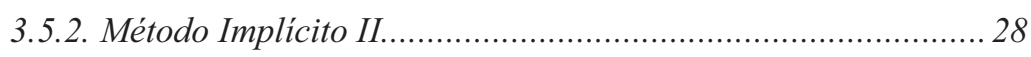

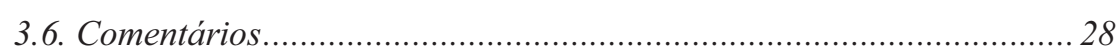

3.6.1. Possíveis problemas na manipulação de matrizes................... 28

3.6.2. Estimativas negativas dos parâmetros da Distribuição

"de Holgate" 
3.6.3. Estimativas não-únicas no método "Chance” I ..................... 30

3.6.4. Outros comentários ................................................ 31

4. Aplicação: Previsões de Resultados de Jogos de Futebol............................... 32

4.1. Dados Utilizados................................................................... 32

4.1.1. Construção do Banco de Dados ......................................... 32

4.1.2. Critérios para inclusão de Jogos ..................................... 32

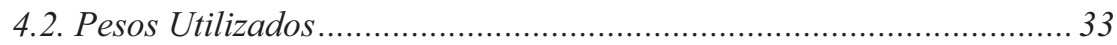

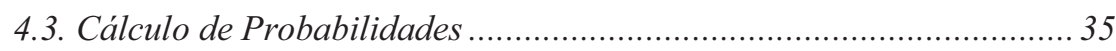

4.4. Site na Internet .................................................................... 35

5. Verificação da Qualidade das Previsões....................................................... 36

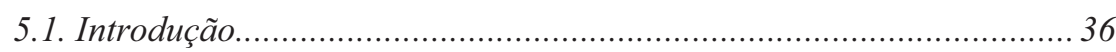

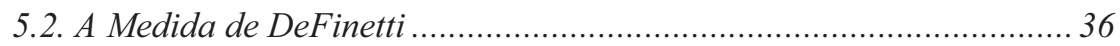

5.3. O Escore de Brier........................................................... 37

5.4. Partições do Escore de Brier............................................................. 38

5.4.1. Abordagem Escalar....................................................... 38

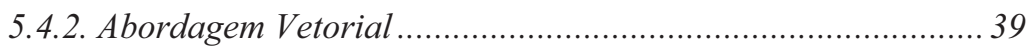

5.5. Gráficos Sugeridos................................................................ 40

5.6. Aplicação das Medidas ao Futebol.............................................. 41

6. Resultados ................................................................................................ 43

6.1. Características particulares de cada método................................. 43

6.2. Resultados das comparações dos métodos ...................................... 44

7. Considerações Finais .................................................................................... 47

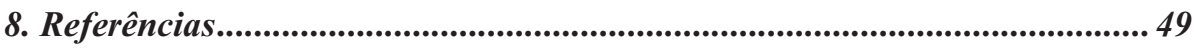

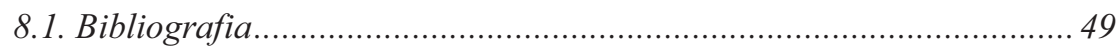

8.2. Sites da Internet .............................................................. 50

A. Previsões Efetuadas pelos Métodos........................................................ 51

A.1. Jogos da Copa do Mundo de 1998 .............................................. 51

A.2. Jogos do Campeonato Brasileiro de 1998.......................................... 64

A.3. Jogos do Torneio Rio-São Paulo de 1999 .................................... 113

B. Curvas de Calibração........................................................................... 119

C. Texto sobre o Site de Previsões para o Campeonato Brasileiro 1999.......... 123 


\section{Capítulo 1 \\ Introdução}

Há diversos artigos, na literatura e na Internet (como por exemplo os sites de RSSSF e WWRR), que, através de procedimentos matemáticos ou estatísticos, produzem valores com o intuito de ordenar equipes de uma determinada modalidade. Tais ordenações podem ser classificadas como rankings (classificações de equipes de acordo com seus resultados históricos, independentemente de sua situação atual) ou como ratings (classificações de equipes de acordo com seu nível técnico atual, independentemente de sua história passada). Todavia, apenas uma parcela diminuta desses artigos se dedica a estimar as probabilidades de cada possível resultado (vitória, empate ou derrota).

O resultado básico de uma partida de futebol (através do qual se estabelece o vencedor e se atribuem pontos na disputa de campeonatos) é, obviamente, seu placar final, o qual deve ser visto como um vetor aleatório bivariado. Pode-se argumentar, teoricamente e apoiando-se na literatura existente, que o número de gols marcados por um time em uma dada partida obedeça a uma distribuição (univariada) de Poisson. Assim, são estudadas as distribuições de Poisson Bivariadas, com destaque para a classe de Holgate. Devido às características particulares do parâmetro covariância, conclui-se que a classe Poísson Bivariada de Holgate é a mais adequada para a modelagem conjunta do placar final de ambos os times.

Conhecida a história (resultados recentes) dos times de cujo confronto se queira modelar o resultado, há 6 métodos propostos para a estimação dos parâmetros da densidade da classe Poisson Bivariada de Holgate. Entre esses métodos há os implícitos (sem utilização explícita de modelagem algébrica e estatística) e os computacionais (baseados em regressão linear ou de Poisson).

De posse de métodos que forneçam as probabilidades de ocorrência de placares, podese aplicar essas estimativas para calcular, por exemplo, a probabilidade de o time A derrotar o time $\mathrm{B}$, a probabilidade de a seleção $\mathrm{C}$ ser campeã, ou a quantidade necessária de pontos que a equipe $\mathrm{D}$ deve conquistar para passar à próxima fase do campeonato.

Previsões de jogos segundo esses métodos foram efetuadas para jogos da Copa do Mundo, do Campeonato Brasileiro de 1998 e do Torneio Rio-São Paulo de 1999, usando como dados históricos, jogos realizados desde 1996, ponderados de acordo com sua antigüidade. Essas previsões foram utilizadas para comparação dos métodos.

Em que pese a ampla literatura existente sobre calibração e verificação de previsões para dados dicotômicos, se encontram poucas referências sobre calibração para dados tricotômicos. Para a verificação da qualidade das previsões efetuadas, utiliza-se a Distância de DeFinetti, além de outros dois métodos sugeridos como expansão das curvas de calibração tradicionais para dados dicotômicos. 


\section{Capítulo 2 As Distribuições de Poisson Bivariadas}

\subsection{A Classe Geral de Distribuições de Poisson Bivariadas}

Nessa dissertação será considerada ponto pacífico a suposição de que o número de gols marcados por um time em uma partida de futebol possua alguma distribuição de Poisson. As conhecidas razões para essa suposição são a unimodalidade da distribuição de Poisson e a própria natureza do processo de Poisson (ver também Seção 2.5). Assim, surge a necessidade da definição de uma distribuição discreta bivariada, através da qual se possa modelar simultaneamente os gols marcados por duas equipes em confronto. Representando-se por $X$ e $Y$ os gols marcados pelas duas equipes, diz-se que o vetor aleatório $(X, Y)$ com suporte $\mathrm{N}^{2}$ segue uma Distribuição de Poisson Bivariada se:

$$
\left\{\begin{array}{l}
\sum_{x=0}^{\infty} \sum_{y=0}^{\infty} P(X=x, Y=y)=1 \\
P(X=x, Y=y) \geq 0, \forall x, y \in\{0,1,2, \ldots\} \\
\sum_{y=0}^{\infty} P(X=x, Y=y)=\frac{e^{-\lambda_{x}}\left(\lambda_{x}\right)^{x}}{x !}, \text { para algum } \lambda_{x}>0 \\
\sum_{x=0}^{\infty} P(X=x, Y=y)=\frac{e^{-\lambda_{y}}\left(\lambda_{y}\right)^{y}}{y !}, \text { para algum } \lambda_{y}>0
\end{array}\right.
$$

A classe de todas as distribuições de Poisson bivariadas é muito ampla (ver Seção 2.4). Para os propósitos desta tese, é suficiente considerar a (sub)classe de distribuições "de Holgate", que em muitos livros é referida como "a" classe de distribuições de Poisson bivariadas. A classe de distribuições bivariadas de Poisson construída por Holgate (1964) é definida como a distribuição conjunta das variáveis $X=P_{1}+P_{12}$ e $Y=P_{2}+P_{12}$, onde $P_{1}, P_{12}$ e $P_{2}$ são três variáveis aleatórias independentes com distribuições de Poisson univariadas.

\subsection{A Classe "de Holgate"}

\subsubsection{Construção da Densidade}

A distribuição de Poisson Bivariada "de Holgate" pode ser construída a partir de três processos de Poisson independentes, sendo $P_{1}, P_{12}$ e $P_{2}$ os números de ocorrências de cada processo durante um período de duração comum (1, por exemplo). As variáveis aleatórias $P_{1}$, $P_{12}$ e $P_{2}$ têm distribuição de Poisson com médias respectivamente iguais a $\lambda_{1}, \lambda_{12}$ e $\lambda_{2}$. Assim, as variáveis $X=P_{1}+P_{12}$ e $Y=P_{2}+P_{12}$ têm distribuição conjunta Poisson bivariada. Claramente, $X$ tem uma distribuição Poisson $\left(\lambda_{1}+\lambda_{12}\right)$, enquanto $Y \sim \operatorname{Poisson}\left(\lambda_{2}+\lambda_{12}\right)$. A densidade conjunta de $X$ e $Y$ pode ser construída assim: 


$$
\begin{aligned}
& P(X=x, Y=y)=P\left(P_{1}+P_{12}=x, P_{2}+P_{12}=y\right)= \\
& =\sum_{i=0}^{\infty} P\left(P_{1}+P_{12}=x, P_{2}+P_{12}=y \mid P_{12}=i\right) P\left(P_{12}=i\right)= \\
& =\sum_{i=0}^{\infty} P\left(P_{1}=x-i, P_{2}=y-i\right) P\left(P_{12}=i\right)= \\
& =\sum_{i=0}^{\min (x, y)} P\left(P_{1}=x-i\right) P\left(P_{2}=y-i\right) P\left(P_{12}=i\right)= \\
& =\sum_{i=0}^{\min (x, y)} \frac{e^{-\lambda_{1}} \lambda_{1}^{x-i}}{(x-i) !} \frac{e^{-\lambda_{2}} \lambda_{2}^{y-i}}{(y-i) !} \frac{e^{-\lambda_{12}} \lambda_{12}^{i}}{i !}= \\
& =e^{-\left(\lambda_{1}+\lambda_{2}+\lambda_{12}\right)} \sum_{i=0}^{\min (x, y)} \frac{\lambda_{1}^{x-i} \lambda_{2}^{y-i} \lambda_{12}^{i}}{(x-i) !(y-i) ! i !} .
\end{aligned}
$$

Hamdan e Al-Bayyati (1969) mostraram que a distribuição de Poisson Bivariada "de Holgate" também pode ser construída através do limite de uma distribuição Binomial Bivariada, de forma semelhante ao que se faz no caso univariado.

Na presença de amostras i.i.d. $\left(X_{1}, Y_{1}\right),\left(X_{2}, Y_{2}\right), \ldots\left(X_{n}, Y_{n}\right)$, os parâmetros $\lambda_{1}, \lambda_{12}$ e $\lambda_{2}$ podem ser estimados através de alguns métodos usuais, mencionados por Kocherlakota \& Kocherlakota (1992) e Johnson, Kotz \& Balakrishnan (1997):

\subsubsection{Formas de Estimação dos Parâmetros com Amostras IID}

Na literatura usual (como Kocherlakota \& Kocherlakota (1992) e Johnson, Kotz \& Balakrishnan (1997)) são mencionados os seguintes métodos de estimação dos parâmetros da Distribuição Poisson Bivariada "de Holgate", dada uma amostra de pares independentes e identicamente distribuídos $\left(X_{1}, Y_{1}\right),\left(X_{2}, Y_{2}\right), \ldots\left(X_{n}, Y_{n}\right)$.

i) Método dos momentos: consiste em usar as relações $E[X]=\lambda_{1}+\lambda_{12}, E[Y]=\lambda_{2}+\lambda_{12} \mathrm{e}$ $\operatorname{Cov}(X, Y)=\lambda_{12}$ para estimar os parâmetros através do sistema de equações

$$
\left\{\begin{array}{l}
\bar{x}=\hat{\lambda}_{1}+\hat{\lambda}_{12} \\
\bar{y}=\hat{\lambda}_{2}+\hat{\lambda}_{12} \\
\operatorname{cov}_{x, y}=\hat{\lambda}_{12}
\end{array}\right.
$$

onde $\operatorname{cov}_{x, y}=\frac{\sum_{i=1}^{n}\left(x_{i}-\bar{x}\right)\left(y_{i}-\bar{y}\right)}{n-1}$ é a covariância amostral entre $X$ e $Y$. 
ii) Método da máxima verossimilhança: consiste em procurar um terno $\left(\hat{\lambda}_{1}, \hat{\lambda}_{2}, \hat{\lambda}_{12}\right)$ que maximize a função $L_{(\mathbf{x}, \mathbf{y})}=\prod_{i=1}^{n} P\left(X=x_{i}, Y=y_{i} \mid \lambda_{1}, \lambda_{2}, \lambda_{12}\right)$. De acordo com Holgate (1964), esse método fornece os estimadores $\hat{\lambda}_{1}+\hat{\lambda}_{12}=\bar{x}$ e $\hat{\lambda}_{2}+\hat{\lambda}_{12}=\bar{y}$, além de uma equação polinomial em $\hat{\lambda}_{12}$. Essa equação não é resolúvel algebricamente e requer solução numérica.

iii) Método do duplo zero: consiste em usar as relações $E[X]=\lambda_{1}+\lambda_{12}, E[Y]=\lambda_{2}+\lambda_{12} \mathrm{e}$ $P(X=0, Y=0)=e^{-\left(\lambda_{1}+\lambda_{2}+\lambda_{12}\right)}$ para estimar os parâmetros através do sistema de equações $\left\{\begin{array}{l}\bar{x}=\hat{\lambda}_{1}+\hat{\lambda}_{12} \\ \bar{y}=\hat{\lambda}_{2}+\hat{\lambda}_{12} \\ -\log p_{0,0}=\hat{\lambda}_{1}+\hat{\lambda}_{2}+\hat{\lambda}_{12}\end{array}\right.$,

onde $p_{0,0}$ é a proporção amostral de pares $(X=0, Y=0)$. (Ver método "Implícito II" na Seção $3.5 .2)$

iv) Outros: na literatura usual também são mencionados o método dos pontos pares (Loukas, Kemp e Papageorgiou, 1986) e o método condicional dos pontos pares (Papageorgiou e Loukas, 1988), cuja filosofia de estimação dos parâmetros foge do escopo desta tese.

\subsubsection{A Identificabilidade dos Parâmetros}

A distribuição de Poisson Bivariada "de Holgate" é totalmente identificável por seus parâmetros, ou seja, é impossível os vetores $(X, Y)$ e $(T, V)$ seguirem distribuições "de Holgate" com parâmetros respectivamente iguais a $\left(\lambda_{1}, \lambda_{2}, \lambda_{12}\right)$ e $\left(v_{1}, v_{2}, v_{12}\right)$, com $\lambda_{i} \neq v_{i}$, para algum $i$ $\underline{\underline{\mathrm{e}}}(X, Y) \sim(T, V)$. Tal fato pode ser demonstrado como segue:

As densidades de $(X, Y)$ e $(T, V)$ são as seguintes:

$$
\begin{gathered}
P(X=r, Y=s)=\frac{e^{-\left(\lambda_{1}+\lambda_{2}+\lambda_{12}\right)} \lambda_{1}^{r} \lambda_{2}^{s}}{r ! s !} \sum_{i=0}^{\min (r, s)}\left(\begin{array}{l}
r \\
i
\end{array}\right)\left(\begin{array}{l}
s \\
i
\end{array}\right) i !\left\{\frac{\lambda_{12}}{\lambda_{1} \lambda_{2}}\right\}^{i} \\
P(T=r, V=s)=\frac{e^{-\left(v_{1}+v_{2}+v_{12}\right)} v_{1}^{r} v_{2}^{s}}{r ! s !} \sum_{i=0}^{\min (r, s)}\left(\begin{array}{l}
r \\
i
\end{array}\right)\left(\begin{array}{l}
s \\
i
\end{array}\right) i !\left\{\frac{v_{12}}{v_{1} v_{2}}\right\}^{i}
\end{gathered}
$$

Então,

a) $(X, Y) \sim(T, V) \Rightarrow P(X=r, Y=s)=P(T=r, V=s) ; \forall r, s \Rightarrow$

$\Rightarrow P(X=0, Y=0)=P(T=0, V=0) \Rightarrow e^{-\left(\lambda_{1}+\lambda_{2}+\lambda_{12}\right)}=e^{-\left(v_{1}+v_{2}+v_{12}\right)} \Rightarrow$

$\Rightarrow \lambda_{1}+\lambda_{2}+\lambda_{12}=v_{1}+v_{2}+v_{12}$; 
b) $(X, Y) \sim(T, V) \Rightarrow P(X=r, Y=s)=P(T=r, V=s) ; \forall r, s \Rightarrow$

$\Rightarrow P(X=1, Y=0)=P(T=1, V=0) \Rightarrow e^{-\left(\lambda_{1}+\lambda_{2}+\lambda_{12}\right)} \lambda_{1}=e^{-\left(v_{1}+v_{2}+v_{12}\right)} v_{1} \Rightarrow$

$\Rightarrow$ (item a) $\lambda_{1}=v_{1}$;

c) $(X, Y) \sim(T, V) \Rightarrow P(X=r, Y=s)=P(T=r, V=s) ; \forall r, s \Rightarrow$

$\Rightarrow P(X=0, Y=1)=P(T=0, V=1) \Rightarrow e^{-\left(\lambda_{1}+\lambda_{2}+\lambda_{12}\right)} \lambda_{2}=e^{-\left(v_{1}+v_{2}+v_{12}\right)} v_{2} \Rightarrow$

$\Rightarrow$ (item a) $\lambda_{2}=v_{2}$.

Por fim, (itens $a, b, c) \Rightarrow\left\{\begin{aligned} \lambda_{1}+\lambda_{2}+\lambda_{12} & =v_{1}+v_{2}+v_{12} \\ \lambda_{1} & =v_{1} \\ \lambda_{2} & =v_{2}\end{aligned} \Leftrightarrow\left\{\begin{array}{c}\lambda_{1}=v_{1} \\ \lambda_{2}=v_{2} \\ \lambda_{12}=v_{12}\end{array}\right.\right.$,

ou seja, dois vetores com distribuição "de Holgate" apresentarão probabilidades iguais (para todo par $(x, y)$ de $\mathrm{N}^{2}$ ) se, e somente se, tiverem distribuições com os mesmos parâmetros.

\subsubsection{Comentários sobre o Parâmetro Covariância}

A covariância entre $X$ e $Y$ pode ser calculada fazendo-se:

$$
\begin{aligned}
\operatorname{Cov}(X, Y) & =\operatorname{Cov}\left(P_{1}+P_{12}, P_{2}+P_{12}\right)= \\
& =\operatorname{Cov}\left(P_{1}, P_{2}\right)+\operatorname{Cov}\left(P_{1}, P_{12}\right)+\operatorname{Cov}\left(P_{2}, P_{12}\right)+\operatorname{Var}\left(P_{12}\right)= \\
& =0+0+0+\lambda_{12}=\lambda_{12} .
\end{aligned}
$$

Como $P(X=0, Y=0)=e^{-\left(\lambda_{1}+\lambda_{2}+\lambda_{12}\right)}$, pode-se perceber que em termos futebolísticos há uma relação direta entre o parâmetro covariância e a probabilidade de ocorrência do resultado $0 \mathrm{x} 0$. Evidentemente, por ser $\lambda_{12}$ o valor esperado da variável $P_{12}$, temse, necessariamente, $\operatorname{Cov}(X, Y) \geq 0$ na classe das distribuições "de Holgate".

A partir da construção por processos de Poisson, pode-se perceber também que, para a classe "Holgate", covariância nula implica independência, pois $\operatorname{Cov}(X, Y)=0$ significa, nessa classe, a inexistência do processo comum $\left(P_{12} \equiv 0\right)$, de onde $X=P_{1}$ e $Y=P_{2}$, independentes.

O Capítulo 3 tratará de métodos de estimação dos parâmetros da Distribuição "de Holgate" para resultados de partidas de futebol, onde não há amostras i.i.d. Esses métodos são apresentados em duas versões: uma em que a covariância é suposta nula (suposição de placares independentes) e outra em que a covariância é estimada juntamente com os demais parâmetros $\left(\lambda_{1}\right.$ e $\left.\lambda_{2}\right)$. As possíveis estimativas negativas para a covariância fornecidas pela segunda versão de algum método de estimação são abordadas na Seção 3.6.2, acompanhadas de sugestões de como contornar tal problema. 


\subsection{Outras Classes Poisson Bivariadas}

\subsubsection{A Classe Poisson Bivariada "de Brooks"}

Uma outra classe de distribuições de Poisson bivariadas existente na literatura é a classe aqui chamada de Poisson Bivariada "de Brooks". Essa classe foi proposta por Johnson e Brooks (1985) em resposta a um problema publicado no American Mathematical Monthly (novembro de 1984), o qual solicitava uma distribuição bivariada de Poisson com covariância negativa. A Distribuição de Poisson Bivariada "de Brooks" pode ser construída da seguinte forma:

Seja $\mathrm{U} \sim U[0,1]$ e definam-se $\quad X_{1}=-\ln \mathrm{U} \quad$ e $\quad X_{2}=-\ln (1-\mathrm{U})$. Então $X_{1} \sim$ Exponencial(1) e $X_{2} \sim$ Exponencial(1). Definam-se agora $\left\{N_{1}(t)\right\}_{t \geq 0}$ e $\left\{N_{2}(t)\right\}_{t \geq 0}$ como processos de contagem com tempos entre chegadas distribuídos de acordo com $X_{1}$ e $X_{2}$, respectivamente. Então, $N_{1}(t) \sim \operatorname{Poisson}(t), N_{2}(t) \sim \operatorname{Poisson}(t)$ e a distribuição conjunta de $N_{1}(t)$ e $N_{2}(t)$ é chamada Distribuição de Poisson Bivariada "de Brooks", e possui $\operatorname{Corr}\left(N_{1}(t), N_{2}(t)\right)<0$.

De acordo com Johnson, Kotz \& Balakrishnan (1997), para intervalos de tamanho $t<\log 2$, são observadas as seguintes particularidades para as probabilidades conjuntas $P\left(N_{1}(t), N_{2}(t)\right)$ :

$$
\begin{aligned}
& P\left(N_{1}(t)>0, N_{2}(t)>0\right)=0 ; \\
& P\left(N_{1}(t)=N_{2}(t)=0\right)=2 e^{-t}-1 ; \\
& P\left(N_{1}(t)=k, N_{2}(t)=0\right)=P\left(N_{1}(t)=0, N_{2}(t)=k\right)=\frac{e^{-t} t^{k}}{k !}(k=1,2,3, \ldots) \\
& \text { e } \operatorname{Corr}\left(N_{1}(t), N_{2}(t)\right)=-t .
\end{aligned}
$$

Por outro lado, para intervalos de tamanho $t>\log 2, P\left(N_{1}(t)=0, N_{2}(t)=0\right)=0$ e, para os demais valores de $\left(N_{1}(t), N_{2}(t)\right)$, a função de probabilidade conjunta pode ser calculada resolvendo-se um conjunto de equações lineares utilizando a simetria da distribuição bivariada; nesses casos o valor da correlação entre $N_{1}(t)$ e $N_{2}(t)$ cresce de - $\log 2$ às proximidades de 0 à medida que o valor de $t$ aumenta.

O gráfico abaixo ilustra essas particularidades. Pode-se perceber, por exemplo, que para valores de $t<\log 2$, certamente ocorrerá $-\log U>t$ (que implica $N_{1}(t)=0$ ) ou $-\log (1-U)>t$ (que implica $\left.N_{2}(t)=0\right)$ e, por conseguinte, $P\left(N_{1}(t)>0, N_{2}(t)>0\right)=0$. Também é fácil perceber que, para valores de $t>\log 2$, certamente ocorrerá $-\log U<t$ (que implica $N_{1}(t)>0$ ) ou $-\log (1-U)<t$ (que implica $N_{2}(t)>0$ ) e, conseqüentemente, $P\left(N_{1}(t)=0, N_{2}(t)=0\right)=0$. As demais particularidades podem ser verificadas de modo análogo. 


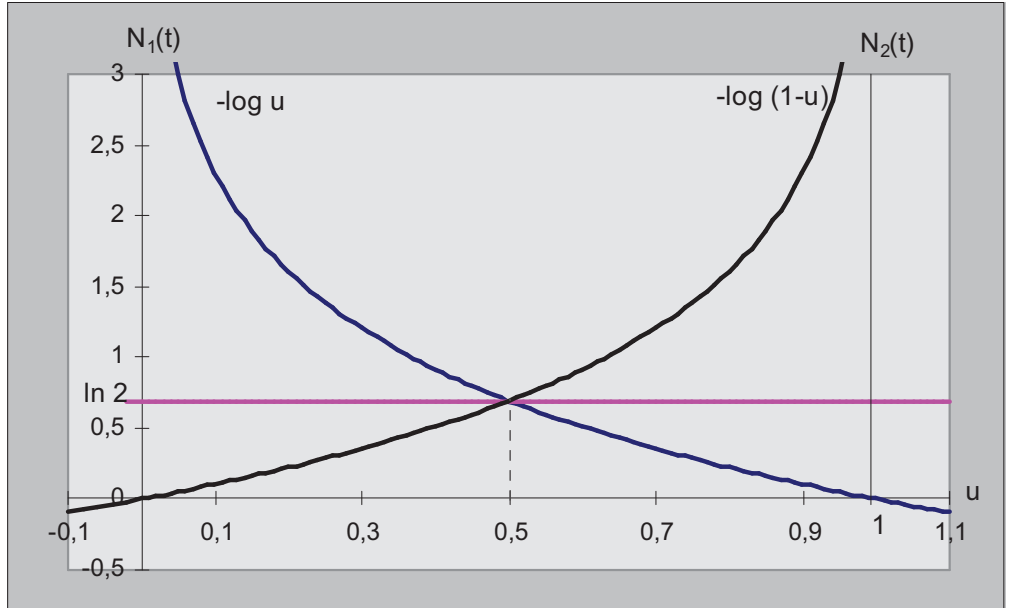

\subsubsection{Uma Classe Poisson Bivariada com Covariância Nula e Dependência}

A existência das distribuições "de Brooks" e "de Holgate" leva à indagação de se é possível construir alguma distribuição bivariada de Poisson com correlação zero e marginais dependentes. A resposta é positiva como pode se ver no exemplo a seguir.

Sejam $X$ e $Y$ com distribuições conjunta e marginais dadas por:

$$
\begin{aligned}
& P(X=0, Y=0)=e^{-\lambda}+e^{-\mu}+\lambda \mu-1, \\
& P(X=1, Y=0)=\lambda e^{-\lambda}-\lambda \mu,
\end{aligned}
$$

Claramente, $X$ e $Y$ têm distribuições marginais $X \sim P(\lambda)$ e $Y \sim P(\mu)$ não independentes.

Aqui, $E[X Y]=\sum_{x=0}^{\infty} \sum_{y=0}^{\infty} x y P(X=x, Y=y)=P(X=1, Y=1)=\lambda \mu$.

Como $E[X] E[Y]=\lambda \mu$, tem-se que $E[X Y]=E[X] E[Y]$, de onde $\operatorname{Cov}(X, Y)=0$.

\subsection{Caracterização da Classe Poisson Bivariada Geral}

Sejam as variáveis independentes $S \sim \operatorname{Poisson}(\lambda)$ e $T \sim \operatorname{Poisson}(\mu)$. Considere-se, também, o vetor $(X, Y)$ com a distribuição construída acima. Nesse caso, as distribuições dos vetores $(S, T)$ e $(X, Y)$ possuem momentos: $E[X]=E[S]=\lambda, \quad E[Y]=E[T]=\mu \quad \mathrm{e}$ $\operatorname{Cov}(X, Y)=\operatorname{Cov}(S, T)=0$. 
Evidentemente, as distribuições dos vetores $(S, T)$ e $(X, Y)$ são extremamente diferentes, o que leva à conclusão de que três parâmetros nem sempre são suficientes para indexar toda a classe de distribuições de Poisson bivariadas. Para pesquisas futuras ficam as perguntas: Quatro parâmetros serão suficientes para caracterizar toda essa classe? Qual seria esse quarto parâmetro? A classe geral seria, então, identificável por seus quatro parâmetros? Existe um número finito de parâmetros para indexar a classe geral?

\subsection{As Classes Poisson Bivariadas e a Previsão de Resultados em Jogos de Futebol}

Dentre as diversas classes de distribuições bivariadas, não são todas as que se adequam à modelagem de resultados de partidas de futebol. Para que uma distribuição bivariada seja considerada adequada, devem ser obedecidos os seguintes critérios:

- As distribuições marginais (gols marcados por cada equipe) devem ser Poisson;

- A distribuição conjunta deve possuir suporte pleno, ao menos perto da origem;

- A distribuição conjunta (e as marginais) devem ser infinitamente divisíveis. De acordo com Dwass e Teicher (1957), um vetor aleatório $\mathbf{X}$ é dito infinitamente divisível se, para qualquer inteiro positivo $n, \mathbf{X}$ tem a mesma distribuição de uma soma de $n$ vetores aleatórios independentes e identicamente distribuídos.

Justifica-se a necessidade da divisibilidade infinita pelo fato de os jogos de futebol, serem disputados em um intervalo contínuo de tempo (diferentemente do vôlei, por exemplo, em que o jogo é dividido em sets que duram o tempo necessário até que um time atinja um número fixado de pontos), que pode ser dividido em intervalos menores, preservando-se suas características probabilísticas. Em termos práticos, isso significa que o resultado de um jogo de futebol pode ser considerado tanto como o resultado de um jogo de 90 minutos, quanto como a soma dos resultados de dois jogos de 45 minutos cada, ou como a soma dos resultados de noventa jogos de 1 minuto cada, etc.

Segundo teorema mencionado por Griffiths et al (1979), toda distribuição bivariada de Poisson infinitamente divisível possui correlação não-negativa. Esse teorema vai de encontro à noção intuitiva de que, quaisquer que sejam duas equipes $\mathrm{X}$ e $\mathrm{Y}$ (que se enfrentem em uma dada partida), estas se enquadram em uma (e apenas uma) das seguintes situações:

$$
\begin{aligned}
& \text { I - X é melhor que Y } \\
& \text { II - X e Y são equivalentes } \\
& \text { III - Y é melhor que X. }
\end{aligned}
$$

Conforme os gráficos de $P(X=x, Y=y)$ abaixo, pode-se perceber que, em qualquer dos casos, a região em que se espera haver maior concentração de massa (probabilidade), indicada pelas linhas pontilhadas, se localiza em torno de retas de regressão com coeficiente angular positivo, o que, probabilisticamente, significa que os números de gols marcados pelas equipes $\mathrm{X}$ e $\mathrm{Y}$ tenham correlação positiva. 


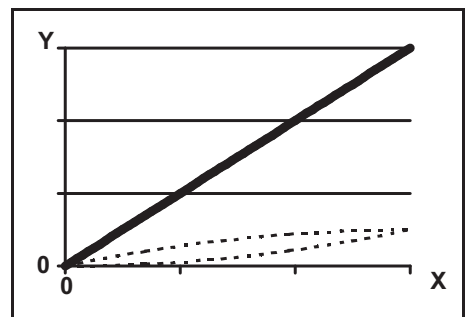

$\mathrm{X}$ muito melhor do que $\mathrm{Y}$

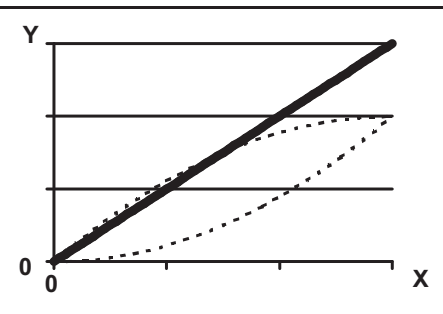

$\mathrm{X}$ moderadamente melhor do que $\mathrm{Y}$ X e $\mathrm{Y}$ equivalentes

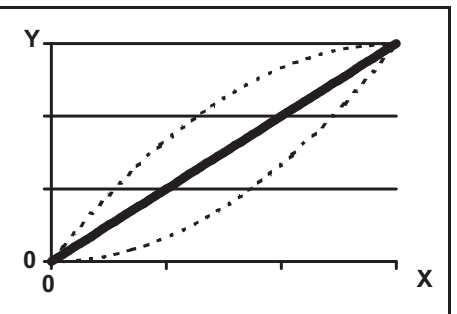

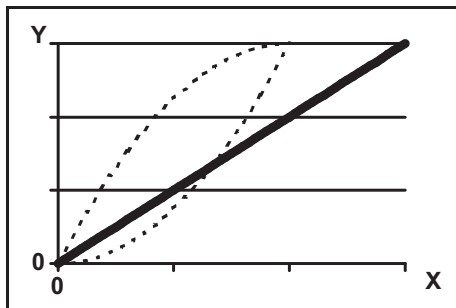

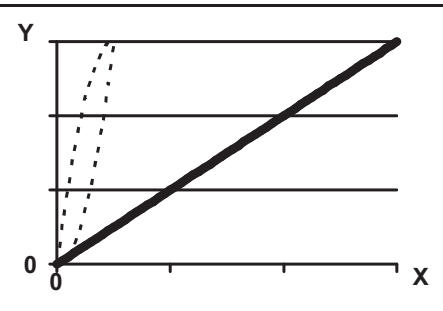

Y muito melhor do que $\mathrm{X}$

A Classe Poisson Bivariada "de Holgate" é bastante adequada à modelagem de resultados de jogos de futebol, pois, além de satisfazer às três condições expostas na página anterior (em particular, possui correlação não-negativa), ela é, na realidade, a única distribuição de Poisson bivariada infinitamente divisível (Dwass e Teicher, 1957). Em outras palavras, a menos que se queira sacrificar as suposições de distribuições marginais Poisson e suporte $\mathrm{N}^{2}$, a classe de Holgate é a única adequada para a modelagem de resultados de jogos de futebol. Assim, no restante desta tese, será adotada a suposição de que os números de gols marcados em qualquer jogo de futebol sigam uma distribuição Poisson Bivariada "de Holgate". 


\section{Capítulo 3 \\ Métodos de Estimação de Parâmetros para Previsão Probabilística de Resultados de Jogos de Futebol}

\subsection{Esclarecimentos Iniciais}

Para uniformização da terminologia utilizada nesta tese, os jogos são escritos sempre na forma Mandante x Visitante, onde "Mandante" é sempre o time que aparecer à esquerda e "Visitante" o que aparecer à direita do sinal x. Em particular, esta convenção será utilizada sempre que um time for de fato "dono da casa" (Mandante) e o adversário estiver "viajando" para disputar a partida (Visitante).

Conforme justificado na Seção 2.5, a distribuição Poisson Bivariada "de Holgate" é bastante apropriada para a modelagem de resultados de partidas de futebol e será utilizada como modelo neste capítulo, onde representa-se o número de gols marcados pelas equipes Mandante e Visitante por $X$ e $Y$, respectivamente. Lembre-se, então, que $E[X]=\lambda_{1}+\lambda_{12}$ e $E[Y]=\lambda_{2}+\lambda_{12}$ são as esperanças marginais dos gols a serem marcados pelas respectivas equipes em um dado jogo.

Outro fato importante utilizado neste capítulo é que, no contexto da distribuição Poisson Bivariada "de Holgate", covariância nula (entre os gols marcados pelas equipes "Mandante" e "Visitante") implica independência. Conforme a construção e a notação utilizadas na Seção 2.2 , é fácil verificar que ${ }^{1}$

$$
\begin{aligned}
& \operatorname{Cov}(X, Y)=0 \Rightarrow \lambda_{12}=0 \Rightarrow E\left[P_{12}\right] \Rightarrow P_{12} \equiv 0 \Rightarrow\left\{\begin{array}{l}
X \equiv P_{1} \\
Y \equiv P_{2}
\end{array} \Rightarrow\right. \\
& \Rightarrow P(X=x, Y=y)=P\left(P_{1}=x, P_{2}=y\right)= \\
& \quad=P\left(P_{1}=x\right) \cdot P\left(P_{2}=y\right)=P(X=x) \cdot P(Y=y) \quad, \quad \forall(x, y) \Rightarrow \\
& \Rightarrow X \mathrm{e} Y \text { independentes. }
\end{aligned}
$$

Por fim, será utilizado, como exemplo de aplicação para todos os métodos apresentados neste capítulo, um torneio hipotético disputado entre Botafogo, Fluminense, Santos e São Paulo, no qual teriam ocorrido os seguintes resultados:

São Paulo 1x0 Santos, em Brasília;

Fluminense 1x2 Botafogo, no Rio de Janeiro;

Botafogo 2x3 São Paulo, no Rio de Janeiro;

Santos 3x2 Fluminense, em Santos;

São Paulo 3x0 Fluminense, em São Paulo;

Santos 2x1 Botafogo, em Santos.

A partida final desse torneio hipotético em turno único, cujo resultado se prevê nos exemplos, será realizada entre os dois melhores colocados, São Paulo e Santos, na cidade de São Paulo.

\footnotetext{
${ }^{1} \mathrm{O}$ símbolo $\equiv$ expressa igualdade com probabilidade 1.
} 


\subsection{A "Transitividade" nos Métodos}

Os métodos apresentados nas próximas duas seções têm em comum a estimação dos parâmetros $\lambda_{1}, \lambda_{2}$ e $\lambda_{12}$ (da distribuição Poisson Bivariada "de Holgate") através de certas funções paramétricas como $E[X+Y], E\left[(X+Y)^{2}\right\rfloor$ ou $P(X=0, Y=0)$. Essa estimação é indireta e isso se deve, antes de mais nada, ao fato de as formas de estimação direta dos parâmetros (como por exemplo as mencionadas na Seção 2.2) dependerem de amostras de variáveis aleatórias independentes, identicamente distribuídas (i.i.d.). No caso específico do futebol, não existem conjuntos (de tamanho razoável) de jogos que, sob algum aspecto, possam ser considerados i.i.d., pois jogos distintos, mesmo que entre os mesmos dois times, envolvem diferenças de comparabilidade devido a diversas características (por exemplo, jogadores, árbitros, condições climáticas e atmosféricas, local e horário de realização da partida, situação das equipes no campeonato etc.). ${ }^{2}$

Além disso, e mais importantemente, métodos baseados em amostras de variáveis aleatórias i.i.d. não possuem a outra propriedade fundamental dos métodos deste capítulo, que pode ser denominada "transitividade entre os jogos". Vários métodos existentes na literatura (como por exemplo, Soares (1982), Lee (1997) e Matthews (1999)) se baseiam em aplicações a competições como o Campeonato Mineiro e o Campeonato Inglês, em que todos os confrontos possíveis entre as equipes participantes acontecem pelo menos uma vez a quase todo ano. No entanto, há muitas competições com jogos de que não se conhecem resultados de confrontos diretos entre as duas equipes envolvidas, mas apenas de confrontos contra outros adversários. Na Copa do Mundo de 1998, por exemplo, houve um jogo entre as seleções de Jamaica e Croácia, que jamais haviam se enfrentado em toda a história. Entretanto, na história recente anterior à Copa do Mundo, houve jogos como Jamaica x Brasil, Brasil x Alemanha e Alemanha x Croácia e Jamaica x México, México x Austrália e Austrália x Croácia.

Assim, os métodos deste capítulo podem prever o resultado de um jogo futuro entre quaisquer duas equipes, mesmo que elas jamais tenham se enfrentado de fato, pois praticamente sempre se pode estimar parâmetros a partir de confrontos "transitivos" como os mencionados no parágrafo acima: se essas equipes tiverem, no passado, enfrentado adversários comuns (ou adversários que tenham enfrentado adversários comuns), e se todos esses confrontos fizerem parte do banco de dados, as estimações podem, então, ser realizadas.

\subsection{Familia de Métodos SD (Soma e Diferença)}

Das propriedades da distribuição Poisson Bivariada "de Holgate", é fácil ver que:

$$
\begin{aligned}
& E[X-Y]=E[X]-E[Y]=\left(\lambda_{1}+\lambda_{12}\right)-\left(\lambda_{2}+\lambda_{12}\right)=\lambda_{1}-\lambda_{2} \quad \mathrm{e} \\
& E[X+Y]=E[X]+E[Y]=\left(\lambda_{1}+\lambda_{12}\right)+\left(\lambda_{2}+\lambda_{12}\right)=\lambda_{1}+\lambda_{2}+2 \lambda_{12} .
\end{aligned}
$$

Esses resultados serão utilizados como base em todos os métodos da Família SD. Esses métodos diferem entre si apenas no que diz respeito à estimação do parâmetro covariância $\left(\lambda_{12}\right)$.

\footnotetext{
${ }^{2}$ A rigor, segundo DeFinetti (1972), não existem sequer dois jogos comparáveis.
} 


\subsubsection{Método SD 0}

Neste método, optou-se pela alternativa mais simples de não estimar a covariância, admitindo-a nula. Tal atitude equivale, como verificado à Seção 3.1, à admissão de independência entre as quantidades de gols marcados pelas equipes Mandante e Visitante de cada jogo. A alternativa de se estimar o valor da covariância será tratada nos métodos SD I e outros.

Assim, pode-se obter a expressão dos valores dos parâmetros de interesse $\lambda_{1} \mathrm{e} \lambda_{2}$ na forma do sistema de equações

$$
\left\{\begin{array}{l}
E[X-Y]=\lambda_{1}-\lambda_{2} \\
E[X+Y]=\lambda_{1}+\lambda_{2}
\end{array}\right.
$$

cuja solução é dada por

$$
\left\{\begin{array}{l}
\lambda_{1}=\frac{E[X-Y]+E[X+Y]}{2} \\
\lambda_{2}=\frac{E[X+Y]-E[X-Y]}{2}
\end{array}\right.
$$

sugerindo os estimadores indiretos

$$
\left\{\begin{array}{l}
\hat{\lambda}_{1}=\frac{\hat{E}[X-Y]+\hat{E}[X+Y]}{2} \\
\hat{\lambda}_{2}=\frac{\hat{E}[X+Y]-\hat{E}[X-Y]}{2}
\end{array}\right.
$$

Por sua vez, $E[X+Y]$ e $E[X-Y]$ são estimados através de modelos lineares dados por

$$
\begin{aligned}
&(X+Y)_{i}=\mathbf{S}_{i} \alpha+\varepsilon_{a i}(4 \mathrm{a}) \\
& \text { e }(X-Y)_{i}=\mathbf{T}_{i} \beta+\varepsilon_{b i}(4 \mathrm{~b}) \\
& \text { (onde: } i=1,2,3, \ldots, n ; n \text { é o número de jogos no banco de dados; } \varepsilon_{a i} \text { e } \varepsilon_{b i} \text { são erros }
\end{aligned}
$$
independentes com médias iguais a 0 .)

Os vetores de parâmetros $\alpha$ e $\beta$ e as covariáveis $\mathbf{S}$ e $\mathbf{T}$ são detalhados a seguir:

No modelo linear (4a), $(X+Y)_{i}$ é o total de gols marcados (por ambas as equipes) no i-ésimo jogo em questão; o vetor $\alpha$ é composto de $\mathrm{N}+1$ parâmetros, sendo um parâmetro associado a cada uma das $\mathrm{N}$ equipes constantes do banco de dados, mais um parâmetro associado ao tipo de local onde o jogo se realiza. Por fim, a matriz-linha $\mathbf{S}_{i}$ possui $\mathrm{N}+1$ elementos, sendo $\mathrm{N}$ associados ao status de cada equipe em relação ao jogo em questão, mais uma componente que indica o tipo de local em que o jogo se realiza. O status de uma equipe é uma variável de incidência que pode assumir os valores 1 se esta participa do i-ésimo jogo ou 0 se não participa. A atribuição comum do valor 1 para as duas equipes envolvidas no jogo se deve ao fato de que o valor de $(X+Y)_{i}$ não depende da identificação de qual equipe seja 
mandante e qual seja visitante: por exemplo, os resultados $3 \times 0,2 \times 1,1 \times 2$ e $0 \times 3$ significam igualmente a ocorrência de três gols $\left((X+Y)_{i}=3\right)$.

A componente relativa ao local de realização do jogo também é uma variável de incidência que pode assumir os valores 1 se o jogo foi no campo do mandante (e estranho ao visitante) ou 0 se foi em campo neutro, seja ele estranho a ambas as equipes (como, por exemplo, o primeiro jogo do torneio hipotético apresentado na Seção 3.1), seja a "casa comum" de ambas as equipes (como o segundo jogo do torneio hipotético).

Os métodos da família SD se baseiam em modelos lineares sem interceptos, o que pode ser justificado pelo fato de as partidas de futebol começarem sempre em 0x0 (e nunca com o placar iniciando em um valor $\beta_{0}$ qualquer).

Para o primeiro jogo do torneio hipotético, por exemplo, $(X+Y)_{1}$ é igual a $1+0=1$

e, sendo o vetor $\alpha$ dado (em ordem alfabética de times, por exemplo) por $\left[\begin{array}{c}\alpha_{B o t} \\ \alpha_{F l u} \\ \alpha_{S a n} \\ \alpha_{S P} \\ \alpha_{\text {Local }}\end{array}\right]$, a matrizlinha $\mathbf{S}_{1}$ torna-se igual a $\left[\begin{array}{lllll}0 & 0 & 1 & 1 & 0\end{array}\right]$.

Ao se considerar conjuntamente todos os jogos do torneio hipotético, $X+Y$ passa a ser o vetor de totais de gols ${ }^{3}$ e $\mathbf{S}$ a matriz de status e local (com uma linha referente a cada jogo, uma coluna referente a cada equipe e a última coluna referente aos locais dos jogos). Para os jogos do torneio hipotético, o modelo (4a) fica dado por

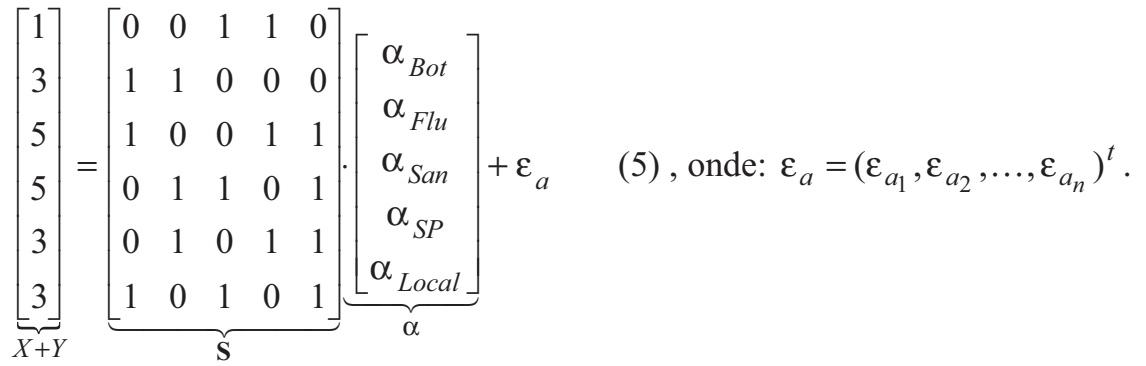

Já no modelo linear $(4 \mathrm{~b}),(X-Y)_{i}$ é a diferença de gols marcados a favor do mandante do jogo $\left(\mathrm{n}^{\mathrm{o}}\right.$ de gols marcados pelo mandante menos $\mathrm{n}^{\mathrm{o}}$ de gols marcados pelo visitante) no i-ésimo jogo do banco de dados; o vetor $\beta$ é composto de $\mathrm{N}+1$ parâmetros, sendo um parâmetro associado a cada uma das $\mathrm{N}$ equipes constantes do banco de dados, mais um parâmetro associado ao tipo de local onde o jogo se realiza. Por fim, a matriz-linha $\mathbf{T}_{i}$ possui $\mathrm{N}+1$ componentes, sendo $\mathrm{N}$ associadas ao status de cada equipe em relação ao jogo em questão, mais uma componente que indica o local em que o jogo se realiza. Nesse modelo, o status de uma equipe é uma variável sinalizada de incidência que pode assumir os valores 1 se esta equipe é a mandante do jogo, -1 se é a visitante ou 0 se esta não participa do i-ésimo

\footnotetext{
${ }^{3}$ Está-se representando, em abuso de notação, o vetor de variáveis dependentes do modelo linear por $X+Y$, ficando claro pelo contexto que não se trata da soma de gols no jogo final ainda a ser jogado. Nessa notação, a soma de gols no jogo final será notada como $(X+Y)_{7}$.
} 
jogo. Aqui, a distinção entre mandante (valor 1) e visitante (valor -1) é necessária pelo fato de o valor de $(X-Y)_{i}$ depender diretamente da identificação de qual equipe seja mandante e qual seja visitante: os resultados $3 \times 0$ e $0 \times 3$ têm significados completamente diferentes (respectivamente, $(X-Y)_{i}=3$ e $\left.(X-Y)_{i}=-3\right)$.

Da mesma forma que no modelo (4a), a componente relativa ao local de realização do jogo também é uma variável de incidência que pode assumir os valores 1 se o jogo foi no campo do mandante (e estranho ao visitante) ou 0 se foi em campo neutro, seja ele estranho a ambas as equipes, seja a "casa comum" de ambas as equipes.

Ainda utilizando-se, como exemplo, o torneio hipotético introduzido na Seção 3.1, tem-se, para o quarto jogo, $(X-Y)_{4}$ igual a 1 e, sendo o vetor $\beta$ dado (em ordem alfabética de times, por exemplo) por

$$
\left[\begin{array}{c}
\beta_{\text {Bot }} \\
\beta_{\text {Flu }} \\
\beta_{\text {San }} \\
\beta_{S P} \\
\beta_{\text {Local }}
\end{array}\right] \text {, a matriz-linha } \mathbf{T}_{4} \text { torna-se igual a }\left[\begin{array}{lllll}
0 & -1 & 1 & 0 & 1
\end{array}\right]
$$

Ao se considerar conjuntamente todos os jogos do torneio, $X-Y$ passa a ser o vetor de diferenças de gols e $\mathbf{T}$ a matriz de status e local (com uma linha referente a cada jogo, uma coluna referente a cada equipe e a última coluna referente aos locais dos jogos). Para os jogos do exemplo, o modelo (4b) fica dado por

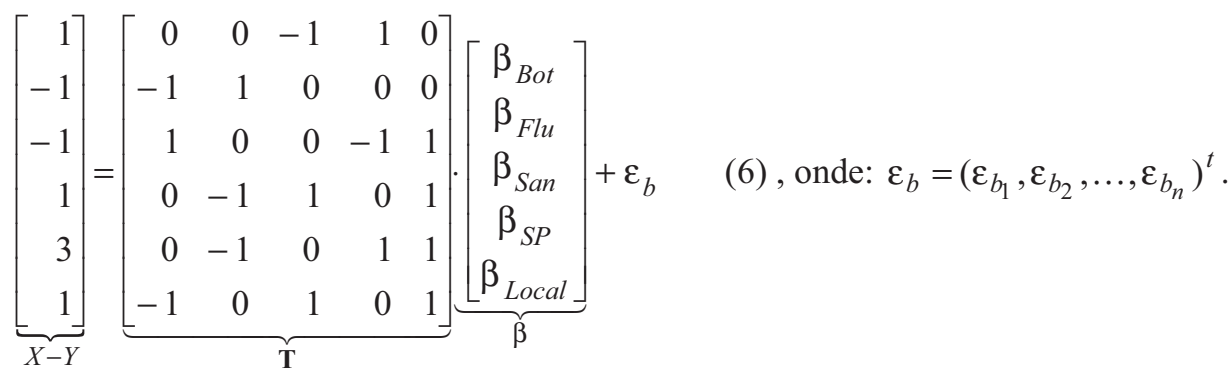

Como se pode perceber, todas as linhas da matriz $\mathbf{T}$ apresentam um valor 1 (associado à equipe mandante), um valor -1 (associado à equipe visitante) e valores 0 associados às demais equipes, fazendo com que sempre exista uma combinação linear das colunas (a soma das colunas relativas às equipes) igual a $(0,0, \ldots, 0)^{\mathrm{t}}$. Logo, a matriz $\mathbf{T}$ sempre possui posto incompleto e, conseqüentemente, T'T é uma matriz singular e não-inversível. Esse aparente problema é abordado e solucionado na Seção 3.6.1, mais adiante.

Os estimadores de $\lambda_{1}$ e $\lambda_{2}$ (para o jogo final São Paulo x Santos) construídos em (3) podem, então, ser calculados a partir de $\hat{E}[X+Y]=\mathbf{S}_{7} \hat{\alpha}$ e $\hat{E}[X-Y]=\mathbf{T}_{7} \hat{\beta}$, onde $\hat{\alpha}$ e $\hat{\beta}$ são obtidos através da minimização (por mínimos quadrados ponderados) da soma dos quadrados das diferenças $(X+Y)_{i}-\mathbf{S}_{i} \alpha$ (modelo $\left.4 \mathrm{a}\right)$ e $(X-Y)_{i}-\mathbf{T}_{i} \beta$ (modelo $4 \mathrm{~b}$ ), respectivamente. A ponderação, cujos pesos serão discutidos posteriormente (na Seção 4.2), é motivada pela necessidade de se dar maior peso aos jogos mais recentes (e, portanto, mais comparáveis ao jogo futuro cujo resultado se quer prever). 
Como exemplo de aplicação, para a final (São Paulo x Santos, em São Paulo) do torneio hipotético, os vetores $\mathbf{S}_{7}$ e $\quad \mathbf{T}_{7}$ são dados por $\mathbf{S}_{7}=\left[\begin{array}{lllll}0 & 0 & 1 & 1 & 1\end{array}\right]$ e $\mathbf{T}_{7}=\left[\begin{array}{lllll}0 & 0 & -1 & 1 & 1\end{array}\right]$, de onde obtêm-se as previsões pontuais

$$
\hat{E}[X+Y]=\mathbf{S}_{7} \hat{\alpha}=\hat{\alpha}_{S a n}+\hat{\alpha}_{S P}+\hat{\alpha}_{\text {Local }}
$$

e $\hat{E}[X-Y]=\mathbf{T}_{7} \hat{\beta}=\hat{\beta}_{S P}-\hat{\beta}_{\text {San }}+\hat{\beta}_{\text {Local }}$, onde $X$ e $Y$ são, respectivamente, o número de gols a serem marcados pelo São Paulo e pelo Santos no jogo final.

Da teoria de mínimos quadrados e de matrizes inversas generalizadas (ver Seção 3.6.1) e admitindo-se com pesos iguais, nesse exemplo - um torneio hipotético de curta duração tem-se as estimativas $\hat{\alpha}$ e $\hat{\beta}$ dadas por:

$$
\hat{\alpha}=\left(\mathbf{S}^{\prime} \mathbf{S}\right)^{-1} \mathbf{S}^{\prime}(X+Y)=\left[\begin{array}{c}
1,5 \\
1,5 \\
0,5 \\
0,5 \\
2
\end{array}\right] \text { e } \hat{\beta}=\left(\mathbf{T}^{\prime} \mathbf{T}\right)^{-} \mathbf{T}^{\prime}(X-Y)=\left[\begin{array}{c}
-1,5 \\
-2,25 \\
-1,25 \\
0 \\
0,5
\end{array}\right]
$$

de onde: $\hat{E}[X+Y]=\mathbf{S}_{7} \hat{\alpha}=\hat{\alpha}_{S a n}+\hat{\alpha}_{S P}+\hat{\alpha}_{\text {Local }}=0,5+0,5+2=3$

$$
\text { e } \hat{E}[X-Y]=\mathbf{T}_{7} \hat{\beta}=\hat{\beta}_{S P}-\hat{\beta}_{\text {San }}+\hat{\beta}_{\text {Local }}=0+1,25+0,5=1,75 \text {. }
$$

Por fim, invocando-se o sistema de equações (3), chega-se às estimativas

$$
\begin{aligned}
& \hat{\lambda}_{S P}=\frac{\hat{E}[X+Y]+\hat{E}[X-Y]}{2}=\frac{3+1,75}{2}=2,375 \mathrm{e} \\
& \hat{\lambda}_{S a n}=\frac{\hat{E}[X+Y]-\hat{E}[X-Y]}{2}=\frac{3-1,75}{2}=0,625 .
\end{aligned}
$$

O resultado $(2,375$ x 0,625) seria o "placar de mínimos quadrados" para o jogo final, mas a informação completa - probabilística - é dada abaixo. A partir dos valores de $\hat{\lambda}_{S P}$ e $\hat{\lambda}_{S a n}$, pode-se construir a matriz de probabilidades "de Holgate" para cada placar, a partir da qual a probabilidade de vitória do São Paulo pode ser calculada como a soma de todos os valores de $P(x, y)$ do triângulo $x>y$. Analogamente, pode-se calcular a probabilidade de empate como a soma de todos os valores de $P(x, y)$ da diagonal $(x=y)$ e a probabilidade de vitória do Santos como a soma de todos os valores de $P(x, y)$ do triângulo $x<y$ :

$$
\begin{aligned}
& P[\text { vitória do São Paulo }]=0,764290 ; \\
& P[\text { empate }]=0,156083 \\
& \text { e } P[\text { vitória do Santos }]=0,079587 .
\end{aligned}
$$




\subsubsection{Método SD I}

Neste método, tem-se a mesma estruturação do método SD 0, incluindo-se agora a estimação da covariância $\lambda_{12}$ entre $X$ e $Y$. Conforme as propriedades das variâncias e da covariância da distribuição "de Holgate", tem-se que

$$
\begin{aligned}
E\left[(X+Y)^{2}\right]-(E[X+Y])^{2} & =\operatorname{Var}[X+Y]= \\
& =\operatorname{Var}[X]+2 \operatorname{Cov}[X, Y]+\operatorname{Var}[Y]= \\
& =\lambda_{1}+\lambda_{12}+2 \lambda_{12}+\lambda_{2}+\lambda_{12} \Rightarrow \\
\Rightarrow E\left[(X+Y)^{2}\right]-(E[X+Y])^{2} & =\lambda_{1}+4 \lambda_{12}+\lambda_{2}
\end{aligned}
$$

Assim, pode-se obter a expressão dos valores dos parâmetros de interesse $\lambda_{1}, \lambda_{2}, \lambda_{12}$ na forma do sistema de equações

$$
\left\{\begin{aligned}
E[X-Y] & =\lambda_{1}-\lambda_{2} \\
E[X+Y] & =\lambda_{1}+\lambda_{2}+2 \lambda_{12} \\
E\left[(X+Y)^{2}\right]-(E[X+Y])^{2} & =\lambda_{1}+4 \lambda_{12}+\lambda_{2}
\end{aligned}\right.
$$

cuja solução é dada por

$$
\left\{\begin{array}{l}
\lambda_{1}=\frac{E[X-Y]+2 E[X+Y]-\left\{E\left[(X+Y)^{2}\right]-(E[X+Y])^{2}\right\}}{2} \\
\lambda_{2}=\frac{2 E[X+Y]-E[X-Y]-\left\{E\left[(X+Y)^{2}\right]-(E[X+Y])^{2}\right\}}{2} \\
\lambda_{12}=\frac{\left\{E\left[(X+Y)^{2}\right]-(E[X+Y])^{2}\right\}-E[X+Y]}{2}
\end{array}\right.
$$

sugerindo os estimadores indiretos

$$
\left\{\begin{array}{l}
\hat{\lambda}_{1}=\frac{\hat{E}[X-Y]+2 \hat{E}[X+Y]-\left\{\hat{E}\left[(X+Y)^{2}\right]-(\hat{E}[X+Y])^{2}\right\}}{2} \\
\hat{\lambda}_{2}=\frac{2 \hat{E}[X+Y]-\hat{E}[X-Y]-\left\{\hat{E}\left[(X+Y)^{2}\right]-(\hat{E}[X+Y])^{2}\right\}}{2} \\
\hat{\lambda}_{12}=\frac{\left\{\hat{E}\left[(X+Y)^{2}\right]-(\hat{E}[X+Y])^{2}\right\}-\hat{E}[X+Y]}{2}
\end{array}\right.
$$


Por sua vez, $E[X+Y], E[X-Y]$ e $E\left[(X+Y)^{2}\right]$ são estimados através dos modelos lineares (4a), (4b) (definidos e explicados na Seção 3.3.1) e (13), dado por

$$
\left[(X+Y)^{2}\right]_{i}=\mathbf{S}_{i} \gamma+\varepsilon_{c i}
$$

(onde: $i=1,2,3, \ldots, n ; n$ é o número de jogos no banco de dados; $\varepsilon_{c i}$ são erros independentes com médias iguais a 0 .)

No modelo linear acima, $\left[(X+Y)^{2}\right]_{i}$ é o quadrado do total de gols marcados (por ambas as equipes) no i-ésimo jogo da amostra; o vetor $\gamma$ é composto por $\mathrm{N}+1$ parâmetros, sendo um parâmetro associado a cada uma das $\mathrm{N}$ equipes constantes do banco de dados, mais um parâmetro associado ao tipo de local onde o jogo se realiza. Por fim, a matriz-linha $\mathbf{S}_{i}$, que possui $\mathrm{N}+1$ elementos, sendo $\mathrm{N}$ associados ao status de cada equipe em relação ao jogo em questão, mais uma componente que indica o tipo de local em que o jogo se realiza, é a mesma definida no modelo (4a). A atribuição indistinta do valor 1 para as duas equipes envolvidas no jogo se deve ao fato de que o valor de $\left[(X+Y)^{2}\right]_{i}$ não depende da identificação de qual equipe seja mandante e qual seja visitante: os resultados $2 \times 0,1 \times 1$ e $0 \times 2$ significam igualmente a ocorrência de dois gols e, conseqüentemente, de $\left[(X+Y)^{2}\right]_{i}=4$.

Para o primeiro jogo do torneio hipotético, por exemplo, $\left[(X+Y)^{2}\right]_{1}$ é igual a $(1+0)^{2}=1 \mathrm{e}$, sendo o vetor $\gamma$ dado, novamente em ordem alfabética de times (por exemplo) por $\left[\begin{array}{c}\gamma_{B o t} \\ \gamma_{F l u} \\ \gamma_{S a n} \\ \gamma_{S P} \\ \gamma_{\text {Local }}\end{array}\right]$, a matriz-linha $\mathbf{S}_{1}$ permanece igual a $\left[\begin{array}{lllll}0 & 0 & 1 & 1 & 0\end{array}\right]$.

Ao se considerar conjuntamente todos os jogos do torneio, $(X+Y)^{2}$ passa a ser o vetor de quadrados de totais de gols ${ }^{4}$ e $\mathbf{S}$ uma matriz de status e local (com uma linha referente a cada jogo, uma coluna referente a cada equipe e a última coluna referente aos locais dos jogos). Para os jogos do exemplo, o modelo (13) fica dado por

\footnotetext{
${ }^{4}$ Está-se representando, em abuso de notação, o vetor de variáveis dependentes do modelo linear por $(X+Y)^{2}$, ficando claro pelo contexto que não se trata do quadrado da soma de gols no jogo final ainda a ser jogado. Nessa notação, o quadrado da soma de gols no jogo final será notado como $\left[(X+Y)^{2}\right]_{7}$
} 


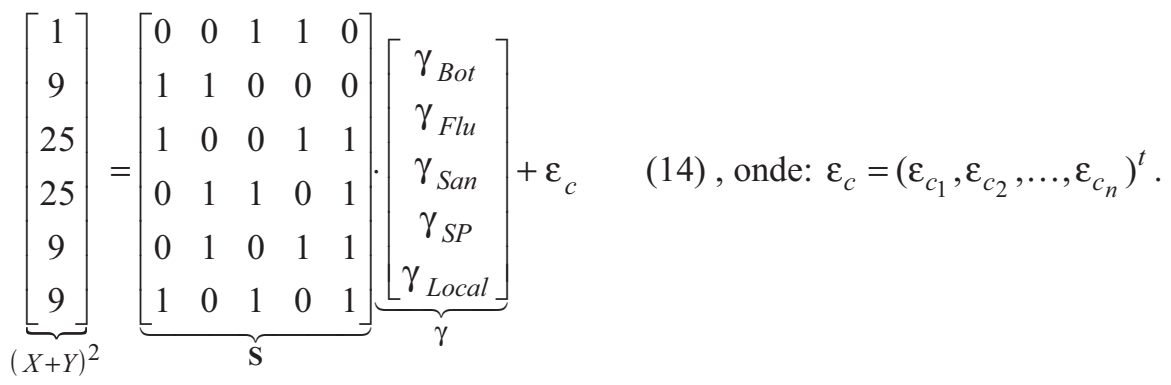

Os estimadores de $\lambda_{1}, \lambda_{2}$ e $\lambda_{12}$ construídos em (12) podem, então, ser obtidos a partir de $\hat{E}[X+Y]=\mathbf{S}_{7} \hat{\alpha}, \hat{E}[X-Y]=\mathbf{T}_{7} \hat{\beta}$ e $\hat{E}\left[(X+Y)^{2}\right]=\mathbf{S}_{7} \hat{\gamma}$, onde $\hat{\alpha}, \hat{\beta}$ e $\hat{\gamma}$ são obtidos através da minimização (por mínimos quadrados ponderados) da soma dos quadrados das diferenças $(X+Y)_{i}-\mathbf{S}_{i} \hat{\alpha}$ (modelo 4a), $(X-Y)_{i}-\mathbf{T}_{i} \hat{\beta}$ (modelo $\left.4 \mathrm{~b}\right)$ e $\left.\mid(X+Y)^{2}\right\rfloor_{i}-\mathbf{S}_{i} \hat{\gamma}$ (modelo 13), respectivamente. A ponderação, cujos pesos serão discutidos posteriormente (na Seção 4.2), é motivada pela necessidade de se dar maior peso aos jogos mais recentes (e, portanto, mais comparáveis ao jogo futuro cujo resultado se quer prever).

Considerando-se o mesmo exemplo de aplicação (a previsão do resultado da final São Paulo x Santos do torneio hipotético), o vetor $\mathbf{S}_{7}$ permanece dado por $\mathbf{S}_{7}=\left[\begin{array}{lllll}0 & 0 & 1 & 1 & 1\end{array}\right]$, de onde obtêm-se o estimador $\hat{E}\left[(X+Y)^{2}\right]=\mathbf{S}_{7} \hat{\gamma}=\hat{\gamma}_{\text {San }}+\hat{\gamma}_{S P}+\hat{\gamma}_{\text {Local }}$ (onde $X$ e $Y$ são o número de gols a serem marcados pelo São Paulo e pelo Santos, na partida final, respectivamente).

Da teoria de mínimos quadrados (ainda com pesos iguais, nesse exemplo), tem-se a estimativa $\hat{\gamma}$ dada por:

$\hat{\gamma}=\left(\mathbf{S}^{\prime} \mathbf{S}\right)^{-1} \mathbf{S}^{\prime}\left[(X+Y)^{2}\right]=\left[\begin{array}{c}4,5 \\ 4,5 \\ 0,5 \\ 0,5 \\ 12\end{array}\right]$, de onde:

$\hat{E}\left[(X+Y)^{2}\right]=\mathbf{S}_{7} \hat{\gamma}=\hat{\gamma}_{S a n}+\hat{\gamma}_{S P}+\hat{\gamma}_{\text {Local }}=0,5+0,5+12=13$

Por fim, invocando-se o sistema de equações (12) e as estimativas $\hat{E}[X+Y]$ e $\hat{E}[X-Y]$ (já obtidas em (8)), chega-se às estimativas: 


$$
\begin{aligned}
\hat{\lambda}_{S P} & =\frac{\hat{E}[X-Y]+2 \hat{E}[X+Y]-\left\{\hat{E}\left[(X+Y)^{2}\right]-(\hat{E}[X+Y])^{2}\right\}}{2}= \\
& =\frac{1,75+2 \cdot 3-\left\{13-3^{2}\right\}}{2}=1,875 \\
\hat{\lambda}_{S a n} & =\frac{2 \hat{E}[X+Y]-\hat{E}[X-Y]-\left\{\hat{E}\left[(X+Y)^{2}\right]-(\hat{E}[X+Y])^{2}\right\}}{2}= \\
& =\frac{2 \cdot 3-1,75-\left\{13-3^{2}\right\}}{2}=0,125 \\
\text { e } \hat{\lambda}_{12}= & \frac{\left\{\hat{E}\left[(X+Y)^{2}\right]-(\hat{E}[X+Y])^{2}\right\}-\hat{E}[X+Y]}{2}=\frac{\left\{13-3^{2}\right\}-3}{2}=0,5,
\end{aligned}
$$

e, conseqüentemente, às probabilidades (obtidas por somas de probabilidades na matriz de "Holgate" de forma igual à descrita na Seção 3.3.1)

$$
\begin{aligned}
& P[\text { vitória do São Paulo }]=0,810830 ; \\
& P[\text { empate }]=0,168962 \\
& \text { e } P[\text { vitória do Santos }]=0,020169 .
\end{aligned}
$$

\subsection{Família de Métodos "Chance"}

Ambos os métodos apresentados nesta seção são inspirados no artigo de Lee, publicado na revista "Chance" em 1997.

\subsubsection{Método "Chance"I}

Neste primeiro método da família, utiliza-se a alternativa mais fiel ao método original de Lee (1997), admitindo-se independência entre os placares $X$ e $Y$ (isto é, o modelo de Holgate com $\left.\lambda_{12}=0\right)$.

Aqui, $E[X]=\lambda_{x}$ e $E[Y]=\lambda_{y}$ são estimados através do modelo log-linear de Poisson (Fahrmeir e Tutz (1994), McCullagh e Nelder (1989) e Paula (1997)) definido como se segue:

Sejam $X_{2 i-1}$ e $X_{2 i}$ os números de gols marcados pelas equipes Mandante e Visitante (respectivamente), no $i$-ésimo jogo $(i=1,2,3, \ldots, n)$. Então, suas distribuições são dadas por

$X_{2 i-1} \sim \operatorname{Poisson}\left(\lambda_{2 i-1}\right)$ e $X_{2 i} \sim \operatorname{Poisson}\left(\lambda_{2 i}\right)$, independentes, e os logaritmos de suas funções de verossimilhança podem, portanto, ser escritos como:

$$
\left\{\begin{array}{c}
\ell\left(\lambda_{2 i-1}, X_{2 i-1}\right)=-\lambda_{2 i-1}+x_{2 i-1} \log \lambda_{2 i-1}-\log \left(x_{2 i-1} !\right) \\
\ell\left(\lambda_{2 i}, X_{2 i}\right)=-\lambda_{2 i}+x_{2 i} \log \lambda_{2 i}-\log \left(x_{2 i} !\right)
\end{array}\right.
$$


O modelo log-linear de Poisson relaciona a distribuição da variável dependente (gols marcados) às variáveis explicativas através da função (canônica) de ligação

$$
\lambda_{j}=e^{\mathbf{U}_{j} \beta}
$$

(onde: $j=1,2,3, \ldots, 2 n ; \mathbf{U}_{1}, \mathbf{U}_{2}, \ldots, \mathbf{U}_{2 n}$ são vetores de covariáveis e $\beta$ é um vetor de parâmetros, explicitados abaixo).

Das expressões (15) e (16), pode-se então escrever as funções de log-verossimilhança como

$$
\left\{\begin{array}{c}
\ell\left(\lambda_{2 i-1}, X_{2 i-1}\right)=-e^{\mathbf{U}_{2 i-1} \beta}+x_{2 i-1} \mathbf{U}_{2 i-1} \beta-\log \left(x_{2 i-1} !\right) \\
\ell\left(\lambda_{2 i}, X_{2 i}\right)=-e^{\mathbf{U}_{2 i} \beta}+x_{2 i} \mathbf{U}_{2 i} \beta-\log \left(x_{2 i} !\right)
\end{array}\right.
$$

Considerando-se a realização de $n$ jogos, o modelo pode ser definido por

$$
\ell\left(\lambda_{1}, \lambda_{2}, \ldots, \lambda_{2 n-1}, \lambda_{2 n}, X_{1}, X_{2}, \ldots, X_{2 k-1}, X_{2 k}\right)=\sum_{j=1}^{2 n}\left(-e^{\mathbf{U}_{j} \beta}+x_{j} \mathbf{U}_{j} \beta-\log \left(x_{j} !\right)\right)
$$

Nesse modelo, $x_{2 i-1}$ e $x_{2 i}$ são os números de gols marcados pelas equipes Mandante e Visitante (respectivamente) no $i$-ésimo jogo; o vetor $\beta$ é composto por $2 \mathrm{~N}+2$ parâmetros, sendo um parâmetro o intercepto, dois parâmetros (um relativo ao ataque e outro à defesa) associados a cada uma das $\mathrm{N}$ equipes constantes do banco de dados, mais um parâmetro associado ao tipo de local onde o jogo se realiza. Por fim, as matrizes-linha $\mathbf{U}_{2 i-1}$ e $\mathbf{U}_{2 i}$ possuem $2 \mathrm{~N}+2$ componentes, sendo a primeira constante e igual a 1 (associada ao intercepto), as $\mathrm{N}$ componentes seguintes associadas ao status de cada equipe em relação ao ataque, as $\mathrm{N}$ componentes subseqüentes associadas ao status de cada equipe em relação à defesa, e a última componente que indica o tipo de local em que o jogo se realiza.

As $\mathrm{N}$ componentes da matriz-linha $\mathbf{U}_{2 i-1}$ relativas ao status das equipes em relação ao ataque, assumirão os valores 1, se a equipe correspondente for a Mandante do $i$-ésimo jogo, ou 0 em caso contrário. Analogamente, as $\mathrm{N}$ componentes relativas ao status das equipes em relação à defesa, assumirão os valores -1 , se a equipe correspondente for a Visitante do $i$-ésimo jogo, ou 0 em caso contrário. Da mesma forma, as $\mathbf{N}$ componentes da matriz-linha $\mathbf{U}_{2 i}$ relativas ao status das equipes em relação ao ataque, assumirão os valores 1 , se a equipe correspondente for a Visitante do $i$-ésimo jogo, ou 0 em caso contrário e as $\mathrm{N}$ componentes relativas ao status das equipes em relação à defesa, assumirão os valores -1 , se a equipe correspondente for a Mandante do $i$-ésimo jogo, ou 0 em caso contrário.

A componente da matriz-linha $\mathbf{U}_{2 i-1}$ relativa ao local de realização do jogo é uma variável indicadora que assume os valores 1 se a equipe Mandante do $i$-ésimo jogo tiver jogado em sua casa, ou 0 em caso contrário. Analogamente, a componente da matriz-linha $\mathbf{U}_{2 i}$ relativa ao local de realização do jogo é uma variável indicadora que assume os valores 1 se a equipe Visitante do $i$-ésimo jogo tiver jogado em sua casa, ou 0 em caso contrário.

Para o primeiro jogo do torneio hipotético, por exemplo, $X_{1}$ e $X_{2}$ são respectivamente iguais a 1 e 0 e, sendo o vetor $\beta$ dado (em ordem alfabética de times e com os parâmetros de ataque colocados antes dos de defesa, por exemplo) por 


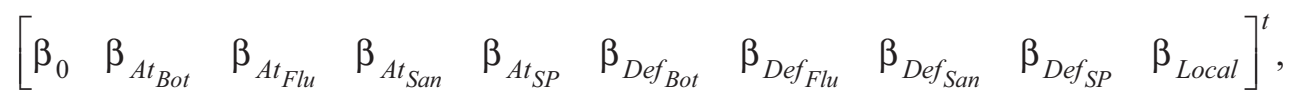

a matriz-linha $\mathbf{U}_{1}$ torna-se igual a $\left[\begin{array}{llllllllll}1 & 0 & 0 & 0 & 1 & 0 & 0 & -1 & 0 & 0\end{array}\right]$ e a matriz-linha $\mathbf{U}_{2}$ torna-se igual a $\left[\begin{array}{llllllllll}1 & 0 & 0 & 1 & 0 & 0 & 0 & 0 & -1 & 0\end{array}\right]$

Ao se considerar, conjuntamente, todos os jogos do torneio hipotético, $\mathbf{U}$ passa a ser uma matriz de constantes (relativas ao intercepto), status e local (com duas linhas referentes a cada jogo, uma coluna referente ao ataque e outra à defesa de cada equipe e a última coluna referente aos locais dos jogos). Para os jogos do torneio hipotético, as matrizes e vetores do modelo (18) ficam dadas por:

$$
\mathbf{U}=\left[\begin{array}{cccccccccc}
1 & 0 & 0 & 0 & 1 & 0 & 0 & -1 & 0 & 0 \\
1 & 0 & 0 & 1 & 0 & 0 & 0 & 0 & -1 & 0 \\
1 & 0 & 1 & 0 & 0 & -1 & 0 & 0 & 0 & 1 \\
1 & 1 & 0 & 0 & 0 & 0 & -1 & 0 & 0 & 1 \\
1 & 1 & 0 & 0 & 0 & 0 & 0 & 0 & -1 & 1 \\
1 & 0 & 0 & 0 & 1 & -1 & 0 & 0 & 0 & 0 \\
1 & 0 & 0 & 1 & 0 & 0 & -1 & 0 & 0 & 1 \\
1 & 0 & 1 & 0 & 0 & 0 & 0 & -1 & 0 & 0 \\
1 & 0 & 0 & 0 & 1 & 0 & -1 & 0 & 0 & 1 \\
1 & 0 & 1 & 0 & 0 & 0 & 0 & 0 & -1 & 0 \\
1 & 0 & 0 & 1 & 0 & -1 & 0 & 0 & 0 & 1 \\
1 & 1 & 0 & 0 & 0 & 0 & 0 & -1 & 0 & 0
\end{array}\right] \text { e } \beta=\left[\begin{array}{c}
\beta_{0} \\
\beta_{A t_{B o t}} \\
\beta_{A t_{F l u}} \\
\beta_{A t_{S a n}} \\
\beta_{A t_{S P}} \\
\beta_{D e f_{B o t}} \\
\beta_{\text {Def Flu }} \\
\beta_{\text {Def }} \\
\beta_{\text {Def }} \\
\beta_{\text {Local }}
\end{array}\right]
$$

Os estimadores de $E\left[X_{2 n+1}\right]=\lambda_{2 n+1}$ e $E\left[X_{2 n+2}\right]=\lambda_{2 n+2}$ podem, então, ser calculados a partir de $\hat{E}\left[X_{2 n+1}\right]=e^{\mathbf{U}_{2 n+1} \hat{\beta}}$ e $\hat{E}\left[X_{2 n+2}\right]=e^{\mathbf{U}_{2 n+2} \hat{\beta}}$, onde $\hat{\beta}$ é obtido através da estimação por regressão de Poisson, com ponderação. Essa ponderação, cujos pesos serão discutidos posteriormente (na Seção 4.2), é motivada pela necessidade de se dar maior peso aos jogos mais recentes (e, portanto, mais comparáveis ao jogo futuro cujo resultado se quer prever).

A regressão de Poisson (Fahrmeir e Tutz (1994), McCullagh e Nelder (1989) e Paula (1997)) é, então, a obtenção do vetor $\hat{\beta}$ que maximize a função de log-verossimilhança (18). Trata-se de uma estimação de máxima verossimilhança onde não existe uma forma analítica fechada para a expressão de $\hat{\beta}$. Essa estimação é, então, realizada através de processos numéricos iterativos ${ }^{5}$.

Considerando-se, o mesmo exemplo de aplicação (a previsão do resultado da final São Paulo x Santos do torneio hipotético), os vetores $\mathbf{U}_{13}$ e $\mathbf{U}_{14}$ ficam dados por

\footnotetext{
${ }^{5}$ Esses processos numéricos iterativos podem levar a estimativas $\hat{\beta}$ diferentes, mas levarão sempre aos mesmos valores das estimativas $\hat{E}\left[X_{j}\right]$. Essa não-unicidade de $\hat{\beta}$ é comentada na Seção 3.6.3.
} 
$\mathbf{U}_{13}=\left[\begin{array}{llllllllll}1 & 0 & 0 & 0 & 1 & 0 & 0 & -1 & 0 & 1\end{array}\right]$ e $\quad \mathbf{U}_{14}=\left[\begin{array}{llllllllll}1 & 0 & 0 & 1 & 0 & 0 & 0 & 0 & -1 & 0\end{array}\right]$, de onde obtêm-se os estimadores $\hat{E}\left[X_{13}\right]=e^{\mathbf{U}_{13} \hat{\beta}}=e^{\hat{\beta}_{0}+\hat{\beta}_{A t_{S P}}-\hat{\beta}_{D e f}+\hat{\beta}_{\text {Local }}}$

$$
\text { e } \hat{E}\left[X_{14}\right]=e^{\mathbf{U}_{14} \hat{\beta}}=e^{\hat{\beta}_{0}+\hat{\beta}_{A t_{S a n}}-\hat{\beta}_{D e f_{S P}}},
$$

onde $X_{13}$ e $X_{14}$ são, respectivamente, o número de gols a serem marcados pelo São Paulo e pelo Santos no jogo final do torneio hipotético.

Através do comando glm ("generalized linear model") do pacote S-Plus, que desenvolve a estimação por meio do algoritmo IWLS ("Iterative Weighted Least Squares") (admitindo-se pesos iguais, nesse exemplo), obtém-se a estimativa $\hat{\beta}$ dada por:

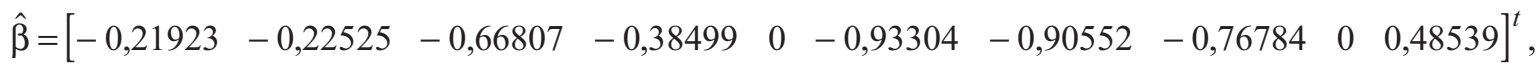

de onde: $\hat{E}\left[X_{13}\right]=e^{\mathbf{U}_{13} \hat{\beta}}=e^{\hat{\beta}_{0}+\hat{\beta}_{A t_{S P}}-\hat{\beta}_{\text {Def San }}+\hat{\beta}_{\text {Local }}}=e^{-0,21923+0-(-0,76784)+0,48539}=2,81229$

$$
\text { e } \hat{E}\left[X_{14}\right]=e^{\mathbf{U}_{14} \hat{\beta}}=e^{\hat{\beta}_{0}+\hat{\beta}_{A t_{S a n}}-\hat{\beta}_{D e f} S P}=e^{-0,21923-0,38499-0}=0,54650
$$

Assim, chega-se às estimativas $\hat{\lambda}_{S P}=2,81229$ e $\hat{\lambda}_{S a n}=0,54650$ e, conseqüentemente, às probabilidades (obtidas por somas de probabilidades na matriz de "Holgate" de forma igual à descrita na Seção 3.3.1):

$P[$ vitória do São Paulo $]=0,839345$;

$P[$ empate $]=0,112630$

e $P[$ vitória do Santos $]=0,047856$.

\subsubsection{Método "Chance" II}

Neste método, tem-se uma estruturação semelhante ao método "Chance I", incluindose agora a estimação da covariância entre $X$ e $Y$. Considerando-se as esperanças marginais dos números de gols marcados pelos dois adversários escritas na forma $E[X]=\lambda_{x}+\lambda_{x y}$ e $E[Y]=\lambda_{y}+\lambda_{x y}$, percebe-se que é possível decompô-las em uma parcela comum $\lambda_{x y}$ e uma parcela $\left(\lambda_{x}\right.$ ou $\left.\lambda_{y}\right)$ que se relaciona somente à distribuição marginal (de $X$ ou de $Y$, respectivamente). Lembrando-se da construção da distribuição "de Holgate" por processos de Poisson (apresentada na Seção 2.2), é possível estabelecer analogias entre a parcela comum e o processo comum e entre as parcelas específicas e os processos específicos das variáveis $X \mathrm{e}$ $Y$.

Considere-se o modelo (22) abaixo, resultado de uma "linearização" do modelo (16), onde $\mathbf{U}^{\prime}$ e $\beta^{\prime}$ são a mesma matriz $\mathbf{U}$ e o mesmo vetor $\beta$ definidos na Seção anterior, alterados apenas com a exclusão da coluna de $\mathbf{U}$ e da componente de $\beta$ relativas ao intercepto: 
$X_{i}=\beta_{0}+\mathbf{U}_{i}^{\prime} \beta^{\prime}+\varepsilon_{b i}$, ou equivalentemente, $E\left[X_{i}\right]=\beta_{0}+\mathbf{U}_{i}^{\prime} \beta^{\prime}$.

(onde: $\varepsilon_{b i}$ são erros independentes com médias iguais a 0 .)

Assim, é possível estabelecer um paralelo entre as notações para $E[X]$ utilizadas na Seção 3.1 e no modelo (22) acima:

$$
E[X]=\underbrace{\lambda_{1}}_{\begin{array}{c}
\text { parcela } \\
\text { especifica }
\end{array}}+\underbrace{\lambda_{12}}_{\begin{array}{c}
\text { parcela } \\
\text { comum }
\end{array}}=\underbrace{\beta_{0}}_{\begin{array}{c}
\text { parcela } \\
\text { comum }
\end{array}}+\underbrace{\mathbf{U}_{i}^{\prime} \beta^{\prime}}_{\begin{array}{c}
\text { parcelas } \\
\text { especificas }
\end{array}}
$$

Então, concluindo-se esse raciocínio baseado em analogias e paralelismos, sugere-se a correspondência $\lambda_{12}=\beta_{0}$.

Assim, pode-se obter a expressão dos valores dos parâmetros de interesse $\lambda_{1}, \lambda_{2}, \lambda_{12}$ na forma do sistema de equações

$$
\left\{\begin{aligned}
E[X] & =\lambda_{1}+\lambda_{12} \\
E[Y] & =\lambda_{2}+\lambda_{12} \\
\beta_{0} & =\lambda_{12}
\end{aligned}\right.
$$

cuja solução é dada por

$$
\left\{\begin{aligned}
\lambda_{1} & =E[X]-\beta_{0} \\
\lambda_{2} & =E[Y]-\beta_{0} \\
\lambda_{12} & =\beta_{0}
\end{aligned}\right.
$$

sugerindo os estimadores indiretos

$$
\left\{\begin{array}{l}
\hat{\lambda}_{1}=\hat{E}[X]-\hat{\beta}_{0} \\
\hat{\lambda}_{2}=\hat{E}[Y]-\hat{\beta}_{0} \\
\hat{\lambda}_{12}=\hat{\beta}_{0}
\end{array}\right.
$$

Por sua vez, $E[X], E[Y]$ (e $\beta_{0}$ ) são estimados através do modelo linear (27), notado como

$$
X_{j}=\mathbf{U}_{j} \beta+\varepsilon_{b j}
$$

(onde: $j=1,2,3, \ldots, 2 n ; \varepsilon_{b j}$ são erros Normais independentes com médias iguais a 0. )

Esse tratamento "linearizado" do modelo utilizado na Seção 3.4.1 se deve ao fato de que a estrutura exponencial utilizada no modelo (16) não permite isolar o intercepto $\beta_{0}$ das demais parcelas, conforme a construção deste método, explicada em (22) e (23). 
No modelo linear (27), $X_{2 i-1}$ e $X_{2 i}$ são os números de gols marcados pelas equipes Mandante e Visitante do $i$-ésimo jogo (da mesma forma como foram definidos na Seção 3.4.1); o vetor $\beta$ e a matriz-linha $\mathbf{U}_{j}$ também são os mesmos definidos na Seção 3.4.1.

Como se pode perceber, todas as linhas da matriz $\mathbf{U}$ apresentam um valor 1 (associado ao ataque de uma equipe), um valor -1 (associado à defesa de outra equipe) e valores 0 associados às demais equipes, fazendo com que sempre exista uma combinação linear das colunas (a soma das colunas relativas aos ataques e às defesas das equipes) igual a $(0,0, \ldots, 0)$. Logo, a matriz $\mathbf{U}$ sempre possui posto incompleto e, conseqüentemente, $\mathbf{U} \mathbf{U}$ é uma matriz singular e não-inversível. Esse aparente problema é abordado e solucionado na Seção 3.6.1, mais adiante.

Ao se considerar, conjuntamente, todos os jogos do torneio hipotético, o vetor $X$ do modelo (27) passa a ser um vetor de gols marcados e fica dado por:

$$
X=\left[\begin{array}{llllllllllll}
1 & 0 & 1 & 2 & 2 & 3 & 3 & 2 & 3 & 0 & 2 & 1
\end{array}\right]^{t}
$$

Sendo o vetor $\beta$ e a matriz $\mathbf{U}$ os mesmos apresentados em (20), os estimadores de $\lambda_{1}$, $\lambda_{2}$ e $\lambda_{12}$ construídos em (26) podem, então, ser calculados a partir de $\hat{E}[X]=\mathbf{U} \hat{\beta}$ e $\lambda_{12}=\hat{\beta}_{0}$, onde $\hat{\beta}$ é obtido através da minimização (por mínimos quadrados ponderados) dos quadrados das diferenças $X_{j}-\mathbf{U}_{j} \hat{\beta}$ (ver modelo 27). A ponderação, cujos pesos serão discutidos posteriormente (na Seção 4.2), é motivada pela necessidade de se dar maior peso aos jogos mais recentes (e, portanto, mais comparáveis ao jogo futuro cujo resultado se quer prever).

Considerando-se, o mesmo exemplo de aplicação (a previsão do resultado da final São Paulo x Santos do torneio hipotético), os vetores $\mathbf{U}_{13}$ e $\mathbf{U}_{14}$ ficam dados por $\mathbf{U}_{13}=\left[\begin{array}{llllllllll}1 & 0 & 0 & 0 & 1 & 0 & 0 & -1 & 0 & 1\end{array}\right]$ e $\mathbf{U}_{14}=\left[\begin{array}{llllllllll}1 & 0 & 0 & 1 & 0 & 0 & 0 & 0 & -1 & 0\end{array}\right]$, de onde obtêm-se os estimadores $\hat{E}\left[X_{13}\right]=\mathbf{U}_{13} \hat{\beta}=\hat{\beta}_{0}+\hat{\beta}_{A t_{S P}}-\hat{\beta}_{D e f_{S a n}}+\hat{\beta}_{\text {Local }}$

$$
\text { e } \hat{E}\left[X_{14}\right]=\mathbf{U}_{14} \hat{\beta}=\hat{\beta}_{0}+\hat{\beta}_{A t_{S a n}}-\hat{\beta}_{D e f_{S P}} \text {, }
$$

onde $X_{13}$ e $X_{14}$ são, respectivamente, o número de gols a serem marcados pelo São Paulo e pelo Santos.

Da teoria de mínimos quadrados (admitindo-se com pesos iguais, nesse exemplo), tem-se que o estimador $\hat{\beta}$ é dado por:

$$
\begin{aligned}
\hat{\beta} & =\left(\mathbf{U}^{\prime} \mathbf{U}\right)^{-} \mathbf{U}^{\prime} X= \\
& =\left[\begin{array}{llllllllll}
0,9444 & 0,2361 & -0,1389 & 0,1111 & 0,7361 & -0,4861 & -0,8611 & -0,1111 & 0,5139 & 0,5
\end{array}\right]^{\prime},
\end{aligned}
$$

de onde:

$$
\hat{E}\left[X_{13}\right]=\mathbf{U}_{13} \hat{\beta}=\hat{\beta}_{0}+\hat{\beta}_{A t_{S P}}-\hat{\beta}_{\text {Def San }}+\hat{\beta}_{\text {Local }}=0,9444+0,7361+0,1111+0,5=2,2916 ;
$$


$\hat{E}\left[X_{14}\right]=\mathbf{U}_{14} \hat{\beta}=\hat{\beta}_{0}+\hat{\beta}_{A t_{\text {San }}}-\hat{\beta}_{\text {Def SP }}=0,9444+0,1111-0,5139=0,5416$

e $\hat{\beta}_{0}=0,9444$

Por fim, invocando o sistema de equações (26), chega-se a:

$$
\begin{aligned}
& \hat{\lambda}_{S P}=\hat{E}\left[X_{13}\right]-\hat{\beta}_{0}=2,2916-0,9444=1,3472, \\
& \hat{\lambda}_{S a n}=\hat{E}\left[X_{14}\right]-\hat{\beta}_{0}=0,5416-0,9444=-0,4028 \\
& \text { e } \hat{\lambda}_{12}=\hat{\beta}_{0}=0,9444 .
\end{aligned}
$$

O aparente problema de $\hat{\lambda}_{\text {San }}$ ser negativo é abordado na Seção 3.6.2. De acordo com essa abordagem, pode-se resolver esse problema com a adoção de $\hat{\lambda}_{\text {San }}=0,25$, chegando-se, finalmente a

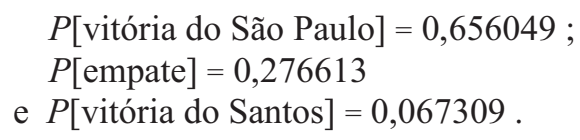

\subsection{Família de Métodos Implícitos ou Diretos}

Esses métodos consistem na expressão direta dos parâmetros sem a utilização explícita de modelagem algébrica e estatística de covariáveis. Esses métodos têm índole bayesiana pouco hierárquica e são motivados pelo conceito de probabilidade subjetiva (Barnett, 1982), segundo o qual, a probabilidade é uma medida de incerteza individual que todo ser humano está capacitado a manifestar na avaliação de situações de incerteza. A escola subjetivista considera ainda que a probabilidade manifestada pela pessoa revela a sua incerteza considerada toda a sua história, isto é, sua experiência, memória e informações. Desse modo, as probabilidades subjetivas declaradas nos métodos implícitos ou diretos incluem de maneira implícita as covariáveis e resultados (e ainda mais informações!) utilizadas nos métodos explícitos anteriores. Isso tudo sugere uma comparação entre métodos implícitos (com prioris declaradas por pessoas que gostem de futebol, em particular, que tenham "boa memória" para resultados e locais de jogos passados) e explícitos, o que é feito no Capítulo 6.

\subsubsection{Método Implícito I}

Nesse método, que parte da suposição (subjetiva) de independência entre os placares, são anunciadas diretamente as estimativas para as esperanças marginais de $X$ e $Y$.

Assim, o vetor de estimativas dos parâmetros $\left(\lambda_{1}, \lambda_{2}, \lambda_{12}\right)$ fica dado por:

$$
\left(\lambda_{1_{\text {anunciado }}}, \lambda_{2_{\text {anunciado }}}, 0\right)
$$

Perceba-se que este método é equivalente a anunciar diretamente uma priori de Holgate para $(X, Y)$. Métodos bayesianos mais hierarquizados anunciariam primeiramente uma priori para $\left(\lambda_{1}, \lambda_{2}\right)$ e, depois, a integração forneceria a priori para $(X, Y)$. A hierarquização pode progredir avançadamente, nem sempre com bons resultados práticos (por 
exemplo, $\lambda_{1} \sim \operatorname{Gama}(a, b)$ independente de $\lambda_{2} \sim \operatorname{Gama}(c, d)$. Por sua vez, $a \sim \operatorname{Expon} .(\theta)$, etc...). De todo modo, a hierarquização aqui é mínima: a rigor, seria mais reduzida somente com o anúncio direto de $P(X=x, Y=y)$, ou mais radicalmente ainda, de $P$ (vitória), $P($ empate) e $P$ (derrota).

\subsubsection{Método Implícito II}

Aqui, além das esperanças marginais, é anunciada a probabilidade $P_{00}$ de ocorrência do resultado $0 \mathrm{x} 0$. $\mathrm{O}$ anúncio da probabilidade $P_{00} \mathrm{em}$ vez de outra função qualquer dos parâmetros se justifica pelo fato que, em geral, as pessoas têm mais facilidade em avaliar e externar a probabilidade de um resultado específico (como o 0x0, por exemplo) do que em avaliar a esperança do produto ou a covariância entre os gols marcados por ambas as equipes. Em outras palavras, a declaração de $\left(E(X), E(Y), P_{00}\right)$ é bem mais adequada, na prática, do que de $(E(X), E(Y), \operatorname{Cov}(X, Y))$ e pode substituir esta última, como é provado abaixo:

Conforme as propriedades da distribuição "de Holgate" e notando-se as esperanças marginais de $X$ e $Y$ por $\mu_{1}$ e $\mu_{2}$, é fácil ver que

$$
P_{00}=P(X=0, Y=0)=e^{-\left(\lambda_{1}+\lambda_{2}+\lambda_{12}\right)}=e^{-\left(\mu_{1}-\lambda_{12}+\mu_{2}-\lambda_{12}+\lambda_{12}\right)}=e^{-\left(\mu_{1}+\mu_{2}-\lambda_{12}\right)},
$$

de onde, $\lambda_{12}=\log P_{00}+\mu_{1}+\mu_{2}$.

Assim, as "estimativas" dos parâmetros $\left(\lambda_{1}, \lambda_{2}, \lambda_{12}\right)$ ficam dadas por:

$$
\left\{\begin{aligned}
\lambda_{1} & =\mu_{1_{\text {anunciado }}}-\lambda_{12} \\
\lambda_{2} & =\mu_{2_{\text {anunciado }}}-\lambda_{12} \\
\lambda_{12} & =\log P_{00_{\text {anunciado }}}+\mu_{1_{\text {anunciado }}}+\mu_{2_{\text {anunciado }}}
\end{aligned}\right.
$$

Surge, contudo, um problema: anunciados os valores $\mu_{1}, \mu_{2}$ e $P_{00}$, o sistema acima pode fornecer um ou mais valores de $\lambda_{1}, \lambda_{2}$ ou $\lambda_{12}$ negativos. Em outras palavras, nem todo trio $\left(\mu_{1}, \mu_{2}, P_{00}\right)$ conduz a valores não-negativos para $\lambda_{1}, \lambda_{2} \mathrm{e} \lambda_{12}$. Subordinado que está o método à adoção da Distribuição de Holgate (ver Seção 2.5), isso significa que há valores $\left(\mu_{1}, \mu_{2}, P_{00}\right)$ que não produzem uma distribuição de probabilidade. Esse fenômeno na teoria Bayesiana é chamado de "incoerência" (na atribuição de valores subjetivos $\mu_{1}, \mu_{2}, P_{00}$ ). Isto é, não há atribuição de uma priori que seja uma distribuição de probabilidades propriamente.

\subsection{Comentários}

\subsubsection{Possíveis problemas na manipulação de matrizes.}

O cálculo envolvido nos métodos SD 0, SD I e "Chance" II (Seções 3.3.1, 3.3.2 e 3.4.2) demanda inversões de matrizes que nem sempre são não-singulares. Pode ocorrer de $\operatorname{det}\left(\mathbf{S}^{\prime} \mathbf{S}\right)$ ser nulo. Além disso, $\operatorname{det}\left(\mathbf{T}^{\prime} \mathbf{T}\right)$ e $\operatorname{det}\left(\mathbf{U}^{\prime} \mathbf{U}\right)$ sempre são nulos, pois as matrizes $\mathbf{T}$ e $\mathbf{U}$, graças à maneira como são construídas, sempre possuem posto incompleto. Para se contornar esse problema, faz-se uso da Matriz Inversa Generalizada de Moore-Penrose: de

\footnotetext{
${ }^{6}$ A rigor, são chamados de "hiperparâmetros" da priori Holgate.
} 
acordo com, por exemplo, Searle (1982) e Seber (1977), diz-se que a matriz G é a (única) inversa generalizada de Moore-Penrose de $\mathbf{X}$ (escreve-se $\mathbf{G}=\mathbf{X}^{-}$) se:
i) $\mathbf{X G X}=\mathbf{X}$;
ii) $\mathbf{G X G}=\mathbf{G}$;
iii) $(\mathbf{X G})^{t}=\mathbf{X G}$;
iv) $(\mathbf{G X})^{t}=\mathbf{G X}$.

Mais geralmente, no que concerne a esta tese, a propriedade mais importante de matrizes inversas generalizadas é a invariância da "previsão" de $\mathbf{X} \hat{\beta}$ a qualquer escolha de $\hat{\beta}$ da forma $\hat{\beta}=\left(\mathbf{X}^{\prime} \mathbf{X}\right)^{-} \mathbf{X}^{\prime} y$ (onde $\left(\mathbf{X}^{\prime} \mathbf{X}\right)^{-}$é alguma inversa generalizada de $\mathbf{X}^{\prime} \mathbf{X}$ ) e, conseqüentemente, a invariância de qualquer contraste $\mathbf{x} \hat{\boldsymbol{\beta}}$, onde $\mathbf{x}$ pertença ao espaço gerado pelas linhas da matriz $\mathbf{X}$.

\subsubsection{Estimativas negativas dos parâmetros da Distribuição "de Holgate".}

É importante perceber que, ao contrário do que ocorre com o método "Chance" I (construído a partir de um modelo de regressão de Poisson), todo o cálculo envolvido nos métodos SD 0, SD I e "Chance" II é baseado em matrizes reais cujos elementos não apresentam qualquer característica que restrinja o valor das soluções $\lambda_{1}, \lambda_{2}$ e $\lambda_{12}$. Assim, em casos particulares é possível que este método forneça estimativas negativas de $\lambda_{1}, \lambda_{2}$ ou $\lambda_{12}$ (da mesma forma que o ocorrido no exemplo da Seção 3.4.2).

Uma solução teórica que se sugere aqui para essas estimativas negativas, é a de uma "caminhada" ou projeção do ponto estimado $\left(\hat{\lambda}_{1}, \hat{\lambda}_{2}, \hat{\lambda}_{12}\right)$ ao ponto mais próximo pertencente ao conjunto de estimativas válidas $\left(R_{+}^{3}\right)$. Algebricamente, isso equivale a igualar a(s) estimativa(s) negativa(s) a zero (conforme explicação a seguir).

No caso do modelo SD 0 (com suposição de independência), o vetor de estimativas é bidimensional $\left(\hat{\lambda}_{1}, \hat{\lambda}_{2}\right)$ e a "caminhada" pode ser graficamente ilustrada, observando-se o gráfico abaixo, onde a região hachurada representa o conjunto das estimativas válidas.

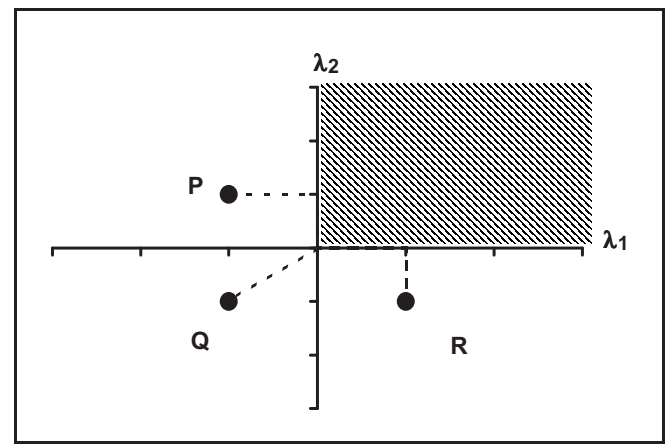

É fácil ver que, para os pontos nos quadrantes pares, como por exemplo $\mathrm{P}\left(\lambda_{1}\right.$ negativo) e $\mathrm{R}$ ( $\lambda_{2}$ negativo), a menor distância que se pode "caminhar" até a região 
hachurada é a representada pela linha pontilhada perpendicular ao semi-eixo positivo adjacente ao ponto em questão, o que equivale a "zerar" a coordenada (estimativa) negativa, mantendo-se inalterada a coordenada (estimativa) positiva.

Para os pontos do terceiro quadrante, como por exemplo $Q$ ( $\lambda_{1} \underline{\mathrm{e}} \lambda_{2}$ negativos), também é fácil perceber que a distância mínima que se pode "caminhar" até a região hachurada é a diagonal pontilhada que liga o ponto em questão até o vértice $(0,0)$, novamente equivalendo ao ato de "zerar" as coordenadas (estimativas) negativas. admissíveis.

Em resumo, trata-se de projetar o ponto externo no conjunto de estimativas

No caso dos modelos SD I e "Chance II", a "caminhada" também pode ser graficamente ilustrada através de um gráfico tridimensional e de observações análogas, sempre coincidindo com a ação de "zerar" as coordenadas (estimativas) negativas.

Para aplicações práticas, entretanto, pode não ser conveniente igualar as estimativas negativas de $\lambda_{1}$ e $\lambda_{2}$ a zero. Invocando notação e construção utilizadas na Seção 2.2, podese ver que, igualar $\lambda_{1}$ (por exemplo) a zero equivale a fazer $P_{1} \equiv 0$ e, conseqüentemente, $X=P_{12}$ e $Y=P_{2}+P_{12}$. Como $P_{12}$ e $P_{2}$ são não-negativos, $Y$ será sempre maior ou igual a $X$, o que significa que $P($ derrota de $Y)=P(X<Y)=0$. No caso do futebol, por exemplo, por maior que seja a diferença entre dois times, nunca se poderá atribuir probabilidade zero à derrota de um deles.

Assim, sugere-se uma "caminhada" alternativa que, em vez de igualar a estimativa negativa a zero, iguale-a a um valor $\varepsilon$ pré-estabelecido (por exemplo, $\varepsilon=0,25$ ).

\subsubsection{Estimativas não-únicas no método "Chance" I}

Uma característica importante que se pode perceber no modelo "Chance" I é que o vetor estimado $\hat{\beta}$ não é único (como também ocorre nas soluções de mínimos quadrados nos métodos SD 0 e SD I). Considere-se, sem perda de generalidade, o jogo São Paulo x Santos, do torneio hipotético. Sendo a modelagem do número de gols de cada time dada por

$$
\hat{E}[X]=e^{\mathbf{U}_{13} \hat{\beta}}=e^{\hat{\beta}_{0}+\hat{\beta}_{A t_{S P}}-\hat{\beta}_{D e f}+\hat{\beta}_{L o c a l}} \text { e } \hat{E}[Y]=e^{\mathbf{U}_{14} \hat{\beta}}=e^{\hat{\beta}_{0}+\hat{\beta}_{A t}{ }_{S a n}-\hat{\beta}_{D e f_{S P}}},
$$

é fácil perceber que as expressões acima podem ser reescritas como se segue (onde $K_{1}$ e $K_{2}$ são constantes quaisquer):

$$
\begin{aligned}
& \hat{E}\left[X_{13}\right]=e^{\mathbf{U}_{13} \hat{\beta}}=e^{\hat{\beta}_{0}+\hat{\beta}_{A t} t_{S P}-\hat{\beta}_{\text {Def }} \text { San }}+\hat{\beta}_{\text {Local }}=e^{\left(\hat{\beta}_{0}+K_{1}+K_{2}\right)+\left(\hat{\beta}_{A t_{S P}}-K_{1}\right)-\left(\hat{\beta}_{\text {Def }}{ }_{\text {San }}+K_{2}\right)+\hat{\beta}_{\text {Local }}}= \\
& =\exp \left\{\hat{\beta}_{0}^{\prime}+\hat{\beta}_{A t_{S P}}^{\prime}-\hat{\beta}_{\text {Def San }}^{\prime}+\hat{\beta}_{\text {Local }}^{\prime}\right\}=\exp \left\{\mathbf{U}_{13} \hat{\beta}^{\prime}\right\} \\
& \text { e } \hat{E}\left[X_{14}\right]=e^{\mathbf{U}_{14} \hat{\beta}}=e^{\hat{\beta}_{0}+\hat{\beta}_{A t_{S a n}}-\hat{\beta}_{D e f_{S P}}}=e^{\left(\hat{\beta}_{0}+K_{1}+K_{2}\right)+\left(\hat{\beta}_{A t_{S a n}}-K_{1}\right)-\left(\hat{\beta}_{D e f} f_{S P}+K_{2}\right)}= \\
& =\exp \left\{\hat{\beta}_{0}^{\prime}+\hat{\beta}_{A t_{\text {San }}}^{\prime}-\hat{\beta}_{D e f_{S P}}^{\prime}\right\}=\exp \left\{\mathbf{U}_{14} \hat{\beta}^{\prime}\right\} \text {. }
\end{aligned}
$$


Entretanto, pode-se notar, também, que embora o vetor $\hat{\beta}$ não seja único, as expressões acima mostram que as estimativas finais $\hat{E}\left[X_{13}\right]$ e $\hat{E}\left[X_{14}\right]$ são sempre únicas, graças às constantes $K_{1}$ e $K_{2}$, que se “cancelam”.

\subsubsection{Outros comentários}

Uma característica bastante útil dos métodos apresentados neste capítulo é o fato de que os estimadores dos parâmetros $\lambda_{1}$ e $\lambda_{2}$ dependem dos valores de $\hat{\alpha}_{\bullet}$ e $\hat{\beta}_{\bullet}$ de ambos os times. Conseqüentemente, a distribuição marginal do número de gols a serem marcados por um dado time varia em função do adversário a ser enfrentado, respeitando a noção futebolística intuitiva de que a performance de um time varie de acordo com o adversário. Essa interdependência entre as distribuições marginais acaba por mostrar a presença (ainda que indiretamente) de uma interação entre as variáveis $X$ e $Y$ (números de gols marcados por cada uma das equipes), o que acaba compensando a eventual suposição de nulidade da covariância. Mais precisamente, mesmo quando $X$ e $Y$ são não-correlacionados, a distribuição de $X$ é específica para o jogo contra o adversário $Y$.

Os métodos explícitos (SD 0, SD I, "Chance" I e "Chance" II) aqui apresentados possuem uma certa parcimônia na escolha das covariáveis incluídas, tornando-os de fácil execução. As (poucas) covariáveis possuem claro significado futebolístico. Por outro lado, um enriquecimento dos modelos lineares deverá considerar, por exemplo, interações entre clube e local, ou mais precisamente, distintos efeitos de mando de campo para diferentes clubes. Na apreciação deste tipo de aumento de modelos, poderá ser considerada a alternativa de testes de significância para interações, por exemplo. Já no estágio dos modelos utilizados neste capítulo, não há testes de significância nem apreciação da qualidade de ajustamentos, justamente por tratarem-se de métodos de estimação pontual de (hiper)parâmetros de prioris Holgate. A apreciação da qualidade das previsões será feita por intermédio de construção de curvas de calibração de probabilidades e outros índices de qualidade (acertos) de previsões probabilísticas, uma vez que todos os métodos deste capítulo são, de alguma forma, de natureza bayesiana, conforme argumentação a seguir.

Os quatro métodos explícitos são, em última análise, bayesianos, uma vez que resultam em uma distribuição de probabilidades para o placar do jogo. O que se questionaria é o fato dos parâmetros destas distribuições não serem (nos métodos explícitos) expressos segundo a mecânica bayesiana, que demanda a combinação de distribuições a priori (para os parâmetros) com verossimilhanças geradas pelas observações amostrais. Como é utilizado exclusivamente o ingrediente amostral nos métodos explícitos, estes são, tecnicamente, métodos bayesianos empíricos (Robbins, 1955). Já os métodos implícitos são integralmente bayesianos, discutindo-se apenas o seu nível de hierarquização. Note-se que a dificuldade de construção de famílias de prioris para $\left(\lambda_{1}, \lambda_{2}\right.$ e $\left.\lambda_{12}\right)$ de manejo matemático simples (de algum modo conjugadas, diga-se) está em paralelo com a dificuldade de obtenção de estimadores baseados em amostras independentes e identicamente distribuídas (ver Capítulo 2). 


\section{Capítulo 4 Aplicação: Previsões de Resultados de Jogos de Futebol}

\subsection{Dados Utilizados}

\subsubsection{Construção do Banco de Dados}

Para a aplicação e posterior avaliação dos métodos apresentados no Capítulo 3, foram efetuadas previsões de resultados (atribuições de probabilidades de placares, de vitória, empate e derrota) para todos os jogos disputados na Copa do Mundo da França, no Campeonato Brasileiro de 1998 e no Torneio Rio-São Paulo de 1999.

Para prever os resultados dos jogos da Copa do Mundo foi utilizado um banco de dados composto por todos os jogos oficiais (campeonatos continentais, eliminatórias de campeonatos continentais, eliminatórias da Copa do Mundo e a Copa das Confederações) realizados entre janeiro de 1996 e julho de 1998, além de amistosos de preparação para a Copa do Mundo realizados no primeiro semestre de 1998. Para a estimação dos parâmetros e previsão dos resultados dos jogos das oitavas-de-finais em diante, os jogos da primeira fase da Copa foram incluídos no banco de dados. ${ }^{7}$

Para a previsão dos resultados dos jogos do Campeonato Brasileiro da Primeira Divisão de 1998, o banco de dados utilizado foi composto por jogos válidos pelo Campeonato Brasileiro de 1997, pelos campeonatos estaduais de 1997 e 1998, pelo Torneio Rio-São Paulo de 1998, pela Copa Nordeste e pela Copa do Brasil de 1998. Para a previsão dos resultados dos jogos do Torneio Rio-São Paulo de 1999, foi utilizado o mesmo banco de dados, sendo apenas removidos os jogos dos campeonatos estaduais de 1997 e acrescentados os jogos do Campeonato Brasileiro da Primeira Divisão de 1998.

Durante a realização do Campeonato Brasileiro de 1998 e do Torneio Rio-São Paulo de 1999, os resultados dos jogos de cada rodada completada foram incluídos no banco de dados para a estimação dos parâmetros e previsão dos resultados dos jogos da rodada seguinte (ver rodapé abaixo).

\subsubsection{Critérios para Inclusão de Jogos}

A grande quantidade de jogos disputados nas competições consideradas na construção do banco de dados para a previsão de resultados nos jogos da Copa do Mundo demandaria um esforço de digitação e análise incompatível com o tempo disponível. Por esse motivo, foi adotado como critério de inclusão de jogos nesse banco de dados a participação de pelo menos uma das 32 seleções classificadas para a Copa do Mundo.

Por motivo análogo, foi adotado como critério de inclusão de jogos no banco de dados para a previsão de resultados do Campeonato Brasileiro, a participação de duas ${ }^{8}$ das 24 equipes presentes na Primeira Divisão de 1998. Como os jogos disputados pelos campeonatos estaduais de 1997 estão consideravelmente distantes do Campeonato Brasileiro de 1998, estes foram incluídos no banco de dados apenas quando envolviam as equipes América (MG) ou Ponte Preta que, por terem disputado o Campeonato Brasileiro de 1997 na Segunda Divisão, participariam de pouquíssimos jogos para o banco de dados.

\footnotetext{
${ }^{7}$ Idealmente, a alimentação do banco de dados deve sempre ser feita após cada jogo, mas razões de ordem prática evidentemente provocam realimentação após grupos de jogos.

${ }^{8}$ Pode-se prescindir de jogos disputados por apenas uma das 24 equipes por razões de abundância de confrontos diretos entre duas dessas equipes.
} 
Também pelo mesmo motivo, para a inclusão de jogos no banco de dados para o Torneio Rio-São Paulo de 1999, foi adotado como critério a participação de duas das 8 equipes presentes na competição.

\subsection{Pesos Utilizados}

Foram encontrados, em sites da Internet sobre estatística e futebol (como os de FIFA, Alex Zheglov e Ron Kessler ® ver Seção 8.2), sistemas de pesos, utilizados na construção de ratings de seleções, os quais têm em comum o fato de a razão entre os pesos de um jogo atual e outro realizado há exatamente três anos e meio (sete semestres) ser igual a 2 . A tabela abaixo mostra os pesos utilizados em cada um dos sites mencionados, além dos pesos $w_{i}$ utilizados nesta tese. Para uniformizar as ordens de grandeza de cada um desses sistemas de pesos, convencionou-se atribuir o valor 1,4 ao peso de um jogo atual.

\begin{tabular}{|c|c|c|c|c|}
\hline Idade do jogo & FIFA & Ron Kessler $^{9}$ & Alex Zheglov ${ }^{10}$ & $\boldsymbol{w}_{i}$ \\
\hline mais de 7 anos & 0 & $(0)$ & 0 & 0 \\
\hline 7 anos $\wp \otimes \otimes 6,5$ anos & 0 & $(0,1)$ & $?$ & $0 \wp \otimes \otimes 0,1$ \\
\hline 6,5 anos $\wp \otimes \otimes 6$ anos & 0 & $(0,2)$ & $?$ & $0,1 \wp \otimes \otimes 0,2$ \\
\hline 6 anos $\wp \otimes \otimes 5,5$ anos & 0,233 & $(0,3)$ & $?$ & $0,2 \wp \otimes \otimes 0,3$ \\
\hline 5,5 anos $\wp \otimes \otimes 5$ anos & 0,233 & $(0,4)$ & $?$ & $0,3 \wp \otimes \otimes 0,4$ \\
\hline 5 anos $\wp \otimes \otimes 4,5$ anos & 0,467 & $(0,5)$ & $?$ & $0,4 \wp \otimes \otimes 0,5$ \\
\hline 4,5 anos $\wp \otimes \otimes 4$ anos & 0,467 & $(0,6)$ & $?$ & $0,5 \wp \otimes \otimes 0,6$ \\
\hline 4 anos $\wp \otimes \otimes 3,5$ anos & 0,7 & 0,7 & $?$ & $0,6 \wp \otimes \otimes 0,7$ \\
\hline 3,5 anos $\wp \otimes \otimes 3$ anos & 0,7 & 0,8 & $?$ & $0,7 \wp \otimes \otimes 0,8$ \\
\hline 3 anos $\wp \otimes \otimes 2,5$ anos & 0,933 & 0,9 & $?$ & $0,8 \wp \otimes \otimes 0,9$ \\
\hline 2,5 anos $\wp \otimes \otimes 2$ anos & 0,933 & 1,0 & $?$ & $0,9 \wp \otimes \otimes 1,0$ \\
\hline 2 anos $\wp \otimes \otimes 1,5$ anos & 1,167 & 1,1 & $?$ & $1,0 \wp \otimes \otimes 1,1$ \\
\hline 1,5 anos $\wp \otimes \otimes 1$ ano & 1,167 & 1,2 & $?$ & $1,1 \wp \otimes \otimes 1,2$ \\
\hline 1 ano $\wp \otimes \otimes 6$ meses & 1,4 & 1,3 & $?$ & $1,2 \wp \otimes \otimes 1,3$ \\
\hline 6 meses ou menos & 1,4 & 1,4 & 1,4 & $1,3 \wp \otimes \otimes 1,4$ \\
\hline
\end{tabular}

Foi utilizado, então, na análise do banco de dados para a Copa do Mundo um sistema de pesos ("combinando" os demais sistemas acima citados e refinando a escala discreta para "dia" como unidade de tempo) dado por:

$$
\begin{aligned}
& w_{i}=1,4-0,4 \frac{M a x-D i a_{i}}{731} \text {, onde: } \\
& w_{i} \text { é o peso do i-ésimo jogo em questão; } \\
& D i a_{i} \text { é a data em que o i-ésimo jogo em questão foi realizado; }
\end{aligned}
$$

\footnotetext{
${ }^{9}$ O rating de Ron Kessler não utiliza jogos com idade superior a 4 anos. Os pesos que aparecem nessa tabelas para jogos anteriores a essa idade foram construídos simplesmente por extensão dos pesos de jogos mais recentes.

${ }^{10}$ No site de Alex Zheglov consta apenas que o peso decresce à medida que o jogo se torna mais antigo, atingindo o valor zero com a idade de 8 anos. Não há informações sobre a regra matemática desse decrescimento.
} 
Max é uma data futura de referência (que pode ser, por exemplo, a data da Final da Copa do Mundo), em relação à qual se calculam as idades dos jogos. Evidentemente, Max deve ser menor ou igual a 7 anos após o jogo mais antigo do banco de dados, pois em caso contrário, surgirão pesos negativos.

Para a análise dos bancos de dados para o Campeonato Brasileiro de 1998 e para o Torneio Rio-São Paulo de 1999, esse sistema de pesos foi ligeiramente alterado, pois os elencos dos times mudam muito mais rapidamente do que os elencos das seleções (Por exemplo, enquanto a Seleção Italiana que disputou a Copa do Mundo de 1998 pode ser considerada basicamente a mesma que iniciou a temporada de 1997, é óbvio que o time do Santos que disputou o Torneio Rio-São Paulo de 1999, é substancialmente diferente daquele que disputou o Campeonato Brasileiro de 1997). Para esses bancos de dados utilizou-se pesos dados por

$$
w_{i}=1,4-0,4\left(\frac{M a x-D i a_{i}}{731}+\frac{s}{2}\right) \text {, onde: }
$$

$w_{i}, D_{i a}$ e Max são os mesmos anteriormente definidos;

$s$ é a idade, em temporadas semestrais completas, do i-ésimo jogo em questão. Para o banco de dados para o Campeonato Brasileiro, por exemplo, os jogos realizados na segunda temporada semestral de 1998 (o próprio Campeonato Brasileiro) têm $s$ igual a zero; os jogos da primeira temporada semestral de 1998 (campeonatos estaduais, Torneio Rio-São Paulo, Copa Nordeste e Copa do Brasil) têm $s$ igual a 1, e assim sucessivamente.

Evidentemente, Max deve ser menor ou igual a 2,5 anos após o jogo mais antigo do banco de dados e $s$ deve ser menor ou igual a 4, pois em caso contrário, surgirão pesos negativos.

A tabela abaixo ilustra a diferença entre os pesos utilizados nos bancos de dados para a Copa do Mundo e para os campeonatos de clubes do Brasil.

\begin{tabular}{|l|c|c|c|}
\cline { 3 - 4 } \multicolumn{2}{c|}{} & \multicolumn{2}{c|}{ Banco de Dados } \\
\hline Idade do jogo & Valor de $\boldsymbol{C}$ & Copa do Mundo & $\begin{array}{c}\text { Campeonato Brasileiro/ } \\
\text { Torneio Rio-São Paulo }\end{array}$ \\
\hline Mais de 7 anos & 14 ou mais & 0 & 0 \\
\hline 7 anos $\wp \otimes \otimes 6,5$ anos & 13 & $0 \wp \otimes \otimes 0,1$ & 0 \\
\hline 6,5 anos $\wp \otimes \otimes 6$ anos & 12 & $0,1 \wp \otimes \otimes 0,2$ & 0 \\
\hline 6 anos $\wp \otimes \otimes 5,5$ anos & 11 & $0,2 \wp \otimes \otimes 0,3$ & 0 \\
\hline 5,5 anos $\wp \otimes \otimes 5$ anos & 10 & $0,3 \wp \otimes \otimes 0,4$ & 0 \\
\hline 5 anos $\wp \otimes \otimes 4,5$ anos & 9 & $0,4 \wp \otimes \otimes 0,5$ & 0 \\
\hline 4,5 anos $\wp \otimes \otimes 4$ anos & 8 & $0,5 \wp \otimes \otimes 0,6$ & 0 \\
\hline 4 anos $\wp \otimes \otimes 3,5$ anos & 7 & $0,6 \wp \otimes \otimes 0,7$ & 0 \\
\hline 3,5 anos $\wp \otimes \otimes 3$ anos & 6 & $0,7 \wp \otimes \otimes 0,8$ & 0 \\
\hline 3 anos $\wp \otimes \otimes 2,5$ anos & 5 & $0,8 \wp \otimes \otimes 0,9$ & $0,1 \wp \otimes \otimes 0,2$ \\
\hline 2,5 anos $\wp \otimes \otimes 2$ anos & 4 & $0,9 \wp \otimes \otimes 1,0$ & $0,4 \wp \otimes \otimes 0,5$ \\
\hline 2 anos $\wp \otimes \otimes 1,5$ anos & 3 & $1,0 \wp \otimes \otimes 1,1$ & $0,7 \wp \otimes \otimes 0,8$ \\
\hline 1,5 anos $\wp \otimes \otimes 1$ ano & 2 & $1,1 \wp \otimes \otimes 1,2$ & $1,0 \wp \otimes \otimes 1,1$ \\
\hline 1 ano $\wp \otimes \otimes 6$ meses & 1 & $1,2 \wp \otimes \otimes 1,3$ & $1,3 \wp \otimes \otimes 1,4$ \\
\hline 6 meses ou menos & 0 & $1,3 \wp \otimes \otimes 1,4$ & \\
\hline
\end{tabular}




\subsection{Cálculo de Probabilidades}

Estimados os parâmetros da distribuição "de Holgate", pode-se calcular a probabilidade de qualquer resultado especifico (por exemplo, $P(2 \mathrm{x} 1)=P(X=2, Y=1)$ ). Conseqüentemente, pode-se calcular a probabilidade dos eventos relativos ao jogo:

$$
\begin{aligned}
& P(\text { vitória })=\sum_{i>j} P(X=i, Y=j) ; P(\text { empate })=\sum_{i} P(X=i, Y=i) \\
& \text { e } P(\text { derrota })=\sum_{i<j} P(X=i, Y=j) .
\end{aligned}
$$

Posteriormente, pode-se calcular a probabilidade de eventos relativos a campeonatos, como por exemplo a probabilidade de o Brasil vencer a Copa do Mundo, a probabilidade de a Portuguesa passar à segunda fase do Campeonato Brasileiro ou a probabilidade de o Goiás não ser rebaixado dado com quantos pontos terminará o campeonato. Como não há fórmulas fechadas para essas probabilidades (Keller (1994) mostrou que os cálculos das probabilidades de empate e vitória envolvem funções de Bessel), as seguintes alternativas podem ser utilizadas:

- Truncar a matriz infinita de resultados possíveis. É suficientemente preciso considerar a matriz $11 \times 11$ (que abrange os resultados de $0 x 0$ a $10 x 10$ ), reduzindo o cálculo das probabilidades de vitória, empate e derrota à soma dos valores correspondentes de $P(X=x, Y=y)$ nessa matriz.

- Simular centenas (ou milhares) de realizações do restante do campeonato em questão, estimando a probabilidade do evento de interesse por sua freqüência relativa de ocorrências.

\subsection{Site na Internet}

Uma aplicação dos modelos de previsão desta tese foi divulgada em um site na Internet (http://www.ime.usp.br/ mlarruda/chgol.html) durante o Campeonato Brasileiro de 1999, onde foram divulgadas probabilidades, periodicamente atualizadas, de classificação para as próximas fases e de rebaixamento para cada time, as probabilidades de vitória, empate e derrota para cada jogo das rodadas seguintes e o número de pontos necessários para que cada time alcançasse a classificação ou evitasse o rebaixamento.

Esse site tem, entre seus objetivos, a divulgação da probabilidade e da estatística ao público leigo, através de um assunto popular como o futebol (Ver, no Apêndice $\mathrm{C}$, texto sobre o site e seus aspectos educativos). 


\section{Capítulo 5 \\ Verificação da Qualidade das Previsões}

\subsection{Introdução}

A bibliografia apresenta ampla teoria sobre calibração e verificação de previsões para eventos dicotômicos (por exemplo, Dawid, 1982 e Kadane \& Lichtenstein, 1982). Entretanto, são encontradas poucas referências sobre verificação de previsões para eventos multinomiais, em que pese a existência de referências para modelos contínuos (como em Loschi, 1992), que não se particularizam imediatamente para modelos finitos, com mais de duas categorias.

Neste capítulo serão utilizados como exemplo de dados tricotômicos, os possíveis resultados de partidas de futebol (vitória, empate ou derrota), cujas probabilidades atribuídas serão representadas por $P V, P E$ e $P D$, respectivamente.

De acordo com Murphy e Winkler (1977), a verificação de previsões ou atribuições de probabilidades abrange três atributos: exatidão (accuracy), confiabilidade (reliability) e qualidade (skill). Em termos informais, a exatidão mede a habilidade de um previsor de realizar uma boa predição pontual, isto é, de aproximar-se do resultado a realizar-se efetivamente (ou do "vértice" a se realizar ® veja a próxima Seção). A exatidão é uma característica pontual do sentido de referir-se a um único jogo (de cada vez). A confiabilidade, diferentemente, mede a habilidade em obter uma média de acertos a longo prazo não muito distante das previsões. Trata-se de uma característica freqüentista, no que se refere aos jogos em conjunto. E o skill mede a superioridade (ou não) do previsor ou método em comparação com algum padrão de referência.

\subsection{A Medida de DeFinetti}

Um método de verificação da qualidade de previsões para eventos tricotômicos bastante difundido na literatura e que será utilizado nesta tese é o de DeFinetti (DeFinetti, 1972), que consiste na consideração de um simplex contido em $\mathrm{R}^{\beta}$ como representação geométrica do conjunto das possíveis previsões probabilísticas. Assim, os vértices desse simplex correspondem às ocorrências dos resultados e os demais pontos a todas as outras possíveis previsões. Formalmente,

$$
S=\left\{(P V, P E, P D) \in \mathrm{R}^{3}: P V+P E+P D=1, P V \geq 0, P E \geq 0, P D \geq 0\right\} .
$$

A medida de distância de DeFinetti corresponde à distância euclideana quadrática entre o ponto correspondente à (distribuição de) probabilidade prevista e o vértice correspondente ao resultado efetivamente observado. Pode-se construir, para mais de uma previsão, um índice dado pela média aritmética das distâncias de DeFinetti, chamado "Medida de DeFinetti".

Para o exemplo de futebol, associam-se os vértices $(1,0,0),(0,1,0)$ e $(0,0,1)$ à vitória da equipe mandante, ao empate e à derrota da equipe mandante, respectivamente. Ao vetor de probabilidades atribuídas para uma determinada partida associa-se o ponto $(P V, P E, P D) \in S$.

Assim, conforme ilustração na próxima página, a distância de DeFinetti será igual a:

$$
\begin{aligned}
& (P V-1)^{2}+(P E-0)^{2}+(P D-0)^{2} \text { se a equipe mandante vencer a partida; } \\
& (P V-0)^{2}+(P E-1)^{2}+(P D-0)^{2} \text { se a partida terminar empatada; }
\end{aligned}
$$

[JPCDA2] Comentário: Form atar em Ekletic 
ou $(P V-0)^{2}+(P E-0)^{2}+(P D-1)^{2}$ se a equipe mandante perder a partida.

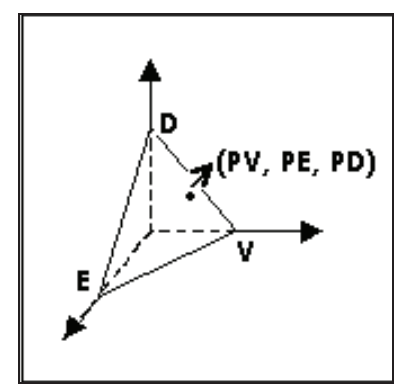

\subsection{O Escore de Brier}

Uma das medidas de exatidão mais utilizadas em verificação de previsões ou atribuições de probabilidades para eventos multinomiais é o Escore de Brier (Brier, 1950), também conhecido como Probability Score (PS) e definido como

$$
P S=\frac{1}{r} \sum_{j=1}^{n} \sum_{i=1}^{r}\left(f_{i j}-E_{i j}\right)^{2}
$$

onde $r$ é o número de observações, $f_{i j}$ é a probabilidade de ocorrência atribuída ao $j$-ésimo resultado na $i$-ésima realização e $E_{i j}$ é uma variável indicadora que assume o valor 1 se $0 j$-ésimo resultado foi observado na $i$-ésima realização e 0 em caso contrário.

No exemplo tricotômico (futebol), sendo realizadas $r$ partidas e $n=3$, tem-se:

$f_{i 1}$ é a probabilidade de vitória da equipe mandante na $i$-ésima partida;

$f_{i 2}$ é a probabilidade de empate na $i$-ésima partida;

$f_{i 3}$ é a probabilidade de derrota da equipe mandante na $i$-ésima partida;

$E_{i 1}=1$ se a equipe mandante venceu na $i$-ésima partida e 0 em caso contrário;

$E_{i 2}=1$ se a equipe mandante empatou na $i$-ésima partida e 0 em caso contrário;

$E_{i 3}=1$ se a equipe mandante perdeu na $i$-ésima partida e 0 em caso contrário.

Nesse caso, em cada partida (i) em que alguma equipe mandante vencer, a contribuição ao Escore de Brier será dada por

$$
\sum_{j=1}^{n}\left(f_{i j}-E_{i j}\right)^{2}=\left(P V_{i}-1\right)^{2}+\left(P E_{i}-0\right)^{2}+\left(P D_{i}-0\right)^{2}
$$

Analogamente, a contribuição ao Escore de Brier de cada partida que terminar empatada será dada por 


$$
\left(P V_{i}-0\right)^{2}+\left(P E_{i}-1\right)^{2}+\left(P D_{i}-0\right)^{2}
$$

e a contribuição ao Escore de Brier de cada partida em que o dono da casa perder será dada por

$$
\left(P V_{i}-0\right)^{2}+\left(P E_{i}-0\right)^{2}+\left(P D_{i}-1\right)^{2} .
$$

Assim, essas expressões mostram que o Escore de Brier é exatamente igual à média das distâncias de DeFinetti.

O Escore de Brier é mais antigo, sendo bastante utilizado para a verificação de previsões meteorológicas desde os anos 50. O Escore de Brier chamou a atenção de DeFinetti que, em diversos artigos (alguns reunidos em DeFinetti, 1972) advogou o seu uso com fins de "correção pedagógica", pois ele julgava que a educação básica deve incluir a avaliação aproximada de incerteza, como é feito com tempo, peso, etc. (DeFinetti, 1963).

O Escore de Brier (e também a medida de DeFinetti) é uma medida de exatidão das previsões, com uma filosofia que premia, a posteriori, as previsões que atribuíram maior probabilidade aos resultados ocorridos. Num exemplo dicotômico, se para $n$ arremessos de moeda forem feitas $n$ previsões iguais a $P($ cara $)=0,8$ e $P($ coroa $)=0,2$, o Escore de Brier ótimo, dadas as previsões, será atingido se forem observadas $100 \%$ de caras e nenhuma coroa.

É fácil perceber que o Escore de Brier será igual a zero se, e somente se, o previsor atribuiu sempre probabilidades iguais a 1 para um resultado e 0 para os demais, e o resultado observado for aquele a que se atribuiu probabilidade 1. Por outro lado, se sempre forem observados algum dos outros dois resultados, a Medida de DeFinetti será igual a 2, seu máximo valor possível.

\subsection{Partições do Escore de Brier}

Uma medida de confiabilidade utilizada por Murphy e Winkler (1977) na verificação de qualidade de previsões ou atribuições de probabilidades pode ser obtida particionando-se o Escore de Brier, conforme efetuado por Murphy (1972a, 1972b). Há duas partições possíveis, cada qual baseada em uma possível abordagem das probabilidades atribuídas para eventos multinomiais. Para ambas as abordagens, trabalhar-se-á com o caso tricotômico e sob a suposição de que o conjunto de valores que as probabilidades atribuídas possam assumir seja finito (discretizado).

\subsubsection{Abordagem Escalar}

A primeira abordagem (abordagem escalar) consiste em tratar a atribuição de probabilidades a $r$ partidas como $3 r$ previsões escalares. Com base nessa abordagem, a partição escalar do Escore de Brier pode ser realizada como segue:

Definam-se $P=\left\{p_{1}, p_{2}, \ldots, p_{m}\right\}$ o conjunto de todos os valores que as probabilidades atribuídas (a vitórias, empates ou derrotas) possam assumir e $r_{i}(i=1,2, \ldots, m)$ o número de atribuições de probabilidades iguais a $p_{i}$. 
Seja $P S_{i}=\frac{1}{r_{i}} \sum_{j_{i}=1}^{r_{i}}\left(p_{i}-E_{j_{i}}\right)^{2} \quad(i=1,2, \ldots, m)$, onde $E_{j_{i}}$ é igual a 1 se o $j$-ésimo evento (dentre aqueles com probabilidade atribuída igual a $p_{i}$ ) ocorreu e 0 em caso contrário.

Então, $P S=\frac{1}{r} \sum_{i=1}^{m} r_{i} P S_{i}$

Como $E_{j_{i}}$ sempre vale 0 ou 1 , tem-se $E_{j_{i}}=E_{j_{i}}^{2}$, de onde:

$P S_{i}=p_{i}^{2}-2 p_{i} \frac{1}{r_{i}} \sum_{j_{i}=1}^{r_{i}} E_{j_{i}}+\frac{1}{r_{i}} \sum_{j_{i}=1}^{r_{i}} E_{j_{i}}=p_{i}^{2}-2 p_{i} F_{i}+F_{i}$

onde $F_{i}$ é a freqüência relativa observada de ocorrência de eventos dentre aqueles cuja probabilidade atribuída foi $p_{i}$.

Completando-se o quadrado na expressão acima, tem-se:

$$
P S_{i}=p_{i}^{2}-2 p_{i} F_{i}+F_{i}^{2}+F_{i}-F_{i}^{2}=\left(p_{i}-F_{i}\right)^{2}+F_{i}\left(1-F_{i}\right)
$$

Por fim, substituindo-se o resultado (3) na expressão (1), tem-se a partição

$$
P S=\frac{1}{r} \sum_{i=1}^{m} r_{i}\left(p_{i}-F_{i}\right)^{2}+\frac{1}{r} \sum_{i=1}^{m} r_{i} F_{i}\left(1-F_{i}\right) \text {. }
$$

Nessa partição, a medida de confiabilidade é a parcela $\frac{1}{r} \sum_{i=1}^{m} r_{i}\left(p_{i}-F_{i}\right)^{2}$, que doravante será referida como "medida escalar de confiabilidade". A outra parcela da partição está associada à resolução (resolution) do previsor.

\subsubsection{Abordagem Vetorial}

A segunda abordagem (abordagem vetorial) consiste em tratar a atribuição de probabilidades a $r$ partidas como $r$ previsões vetoriais. Com base nessa abordagem, a partição vetorial do Escore de Brier pode ser realizada como segue (em termos probabilísticos, essa abordagem é mais correta, pois, para cada jogo, a previsão é uma distribuição de probabilidades):

Definam-se $P=\left\{\mathbf{p}_{1}, \mathbf{p}_{2}, \ldots, \mathbf{p}_{m}\right\} \quad$ o conjunto de todos os distintos vetores de probabilidades que foram atribuídos (a vitórias, empates e derrotas) possam assumir e $r_{i}$ $(i=1,2, \ldots, m)$ o número de atribuições de probabilidade $\mathbf{p}_{i}$.

Então, procedendo de forma análoga ao cálculo da partição escalar, obtêm-se a partição 


$$
P S=\frac{1}{r} \sum_{i=1}^{m} r_{i}\left(\mathbf{p}_{i}-\mathbf{F}_{i}\right)\left(\mathbf{p}_{i}-\mathbf{F}_{i}\right)^{\prime}+\frac{1}{r} \sum_{i=1}^{m} r_{i} \mathbf{F}_{i}\left(\mathbf{u}-\mathbf{F}_{i}\right)^{\prime},
$$

onde $\mathbf{F}_{i}$ é o vetor (linha) de freqüências relativas observadas nos eventos com probabilidade atribuída igual a $\mathbf{p}_{i}$ e u é um vetor (linha) unitário de tamanho $n$ (no exemplo de futebol, $\mathbf{u}=(1,1,1))$.

Nessa partição, a medida de confiabilidade é a parcela $\frac{1}{r} \sum_{i=1}^{m} r_{i}\left(\mathbf{p}_{i}-\mathbf{F}_{i}\right)\left(\mathbf{p}_{i}-\mathbf{F}_{i}\right)^{\prime}$, que doravante será referida como "medida vetorial de confiabilidade". A outra parcela da partição está associada à resolução (resolution) do previsor.

Ambas as medidas de confiabilidade construídas nesta seção são medidas do "senso probabilístico freqüentista" do previsor, tendo uma filosofia que premia as previsões que tenham atribuído probabilidades mais próximas das freqüências relativas ocorridas. No exemplo dicotômico, se para $n$ arremessos de moeda forem feitas $n$ previsões iguais a $P($ cara $)=0,8$ e $P($ coroa $)=0,2$, as medidas de confiabilidade ótimas serão atingidas se forem observadas $80 \%$ de caras e $20 \%$ de coroas. Compare-se com as medidas ótimas de exatidão (Seção 5.3).

As parcelas associadas à resolução (em ambas as partições) têm significado similar a uma medida de variabilidade das freqüências observadas. Essas parcelas não dependem diretamente das previsões, mas indiretamente sim, pois é o previsor quem define $m$ e $\left(r_{1}, r_{2}, \ldots, r_{m}\right)$. Assim, as parcelas associadas à resolução não serão utilizadas explicitamente como ferramentas na verificação e avaliação dos métodos definidos no Capítulo 3.

Dadas as previsões, as parcelas associadas à resolução não são fixas (pois dependem de $F_{i}$ ou $\mathbf{F}_{i}$, os quais dependem dos placares) e só resta ao previsor "torcer" para que os resultados dos jogos venham a fornecer calibração e exatidão boas (i.e. primeira parcela da partição e Escore de Brier pequenos).

\subsection{Gráficos Sugeridos}

Assim como a Medida de DeFinetti está associada à representação geométrica das previsões como pontos no simplex, as medidas de confiabilidade construídas na Seção anterior também sugerem representações gráficas através das quais se possa visualizar as características de confiabilidade (ou "calibração" $\otimes$ ver Dawid, 1982) do método de previsão.

A medida escalar de confiabilidade (Seção 5.4.1) pode ser encarada como a média ponderada (com pesos $r_{i}$ ) das diferenças $\left(p_{i}-F_{i}\right)^{2}$. Isso sugere a utilização de um gráfico de $f\left(p_{i}\right)=F_{i}$, onde a cada valor de probabilidade atribuída se associa a respectiva freqüência relativa observada de eventos. Evidentemente, nesse gráfico, a confiabilidade perfeita (ou a concordância perfeita entre probabilidades atribuídas e freqüências observadas) é dada por $f\left(p_{i}\right)=p_{i}, \forall p_{i}$ e representada pela reta identidade e a medida de confiabilidade pode ser 
interpretada como a média ponderada (com pesos $r_{i}$ ) das Distâncias Euclideanas Quadráticas entre a curva do previsor e a reta identidade.

Analogamente, a medida vetorial de confiabilidade (Seção 5.4.2) pode ser encarada como a média ponderada (com pesos $r_{i}$ ) das diferenças $\left(\mathbf{p}_{i}-\mathbf{F}_{i}\right)\left(\mathbf{p}_{i}-\mathbf{F}_{i}\right)^{\prime}$, sugerindo a utilização de um gráfico de $f\left(\mathbf{p}_{i}\right)=\mathbf{F}_{i}$, onde a cada vetor de probabilidades atribuídas se associam os respectivos vetores de freqüências observadas de eventos. Nesse gráfico, a confiabilidade perfeita (ou a concordância perfeita entre probabilidades atribuídas e freqüências observadas) é dada por $f\left(\mathbf{p}_{i}\right)=\mathbf{p}_{i}, \forall \mathbf{p}_{i}$. No exemplo de futebol, essa concordância perfeita pode representada pela figura plana $(p, q, 1-p-q, p, q, 1-p-q)$ imerso em $[0,1]^{6}$ e a medida de confiabilidade pode ser interpretada como a média ponderada (com pesos $r_{i}$ ) das Distâncias Euclideanas Quadráticas entre a figura do previsor (do tipo ( $p, q, 1-p-q, x, y, 1-x-y$ ), quadridimensional) e o citada figura plana.

O gráfico sugerido pela medida vetorial de confiabilidade exige o desenho de figuras quadridimensionais em espaços hexadimensionais, o que é computacionalmente inviável e impede seu uso nesta tese. Além disso, qualquer que seja a discretização utilizada, a utilização da medida vetorial implica, para a construção de seu gráfico, a utilização de classes mais numerosas e mais rarefeitas do que no gráfico da medida escalar: a uma discretização que utilize $c$ classes no gráfico da medida escalar, Murphy (1972a, 1972b) calcula corresponder, no gráfico da medida vetorial, uma quantidade de classes dada por

$$
T=\sum_{i=1}^{c}\left(\begin{array}{c}
n+i-4 \\
i-1
\end{array}\right)(c-i+1), \quad \text { para } n \geq 3 .
$$

Particularmente nesta tese, foram atribuídas probabilidades a $r=390$ jogos e utilizou-se a discretização com intervalos centrados em $0,05,0,15,0,25, \ldots, 0,85,0,95$. Essa discretização equivale à utilização de 10 classes no gráfico da medida escalar, com média de $(390 * 3) / 10=87$ dados por classe. Por outro lado, para o gráfico da medida vetorial, essa discretização corresponde à utilização de $T=55$ classes, com média de $390 / 15=7,091$ dados por classe.

Por todos esses motivos, serão utilizados nesta tese a medida escalar de confiabilidade e seu respectivo gráfico. Ao contrário do que intuitivamente pode parecer, o uso da medida escalar não incorre em redundância, pois pode-se provar que esse gráfico não é equivalente a qualquer outro que possa ser construído considerando apenas dois resultados (vitórias e empates, por exemplo). No Apêndice $\mathrm{B}$, encontram-se os gráficos (também referidos como "curvas de calibração" $\otimes$ ver Dawid, 1982) construídos para cada um dos métodos descritos no Capítulo 3, com base nos jogos especificados na Seção 4.1.

É importante observar, na construção deste gráfico, o critério utilizado para discretização dos dados, principalmente quando a quantidade de eventos previstos e observados for relativamente pequena. Assim como na construção de histogramas, a discretização em intervalos muito pequenos pode levar a intervalos com poucas observações, enquanto a utilização de intervalos grandes pode ocultar alguma característica importante dos dados ao aglomerar muitas observações em um único intervalo. 


\subsection{Aplicação das Medidas ao Futebol}

Conforme mencionado na Seção 5.1, a verificação de previsões ou atribuições de probabilidades se faz mediante três atributos (Murphy e Winkler, 1977): exatidão (accuracy), confiabilidade (reliability) e qualidade (skill).

Como medidas numéricas dos dois primeiros atributos, serão utilizadas nesta tese para comparações (feitas no Capítulo 6) dos métodos apresentados no Capítulo 3:

- Exatidão: Média aritmética das distâncias de DeFinetti, equivalente ao Escore de Brier (definida nas Seções 5.2 e 5.3). A expressão da distância de DeFinetti em termos futebolísticos já foi apresentada na Seção 5.2.

- Confiabilidade: Medida escalar de confiabilidade (definida nas Seções 5.4.1 e 5.5). Pode ser expressa, em termos futebolísticos, como:

$$
\begin{aligned}
M e C & =\frac{1}{r} \sum_{p} r_{p}(f(p)-p)^{2}=\frac{\sum_{p} r_{p}(f(p)-p)^{2}}{\sum_{p} r_{p}}= \\
& =\frac{\sum_{p}\left(\# V P_{p}+\# E P_{p}+\# D P_{p}\right)\left(\frac{\# V O_{p}+\# E O_{p}+\# D O_{p}}{\# V P_{p}+\# E P_{p}+\# D P_{p}}-p\right)^{2}}{\sum_{p}\left(\# V P_{p}+\# E P_{p}+\# D P_{p}\right)}, \text { onde: }
\end{aligned}
$$

$\# V O_{P}=$ número de vitórias (com probabilidade $p$ atribuída) ocorridas; $\# E O_{P}=$ número de empates (com probabilidade $p$ atribuída) ocorridos; $\# D O_{P}=$ número de derrotas (com probabilidade $p$ atribuída) ocorridas; $\# V P_{P}=$ número de vitórias (com probabilidade $p$ atribuída) previstas; $\# E P_{P}=$ número de empates (com probabilidade $p$ atribuída) previstos; $\# D P_{P}=$ número de derrotas (com probabilidade $p$ atribuída) previstas.

O terceiro atributo (qualidade), pode ser avaliado simplesmente com a comparação entre o valor obtido da medida de exatidão (Medida de DeFinetti/Escore de Brier) e o valor da medida calculado para algum padrão de interesse. Para dados tricotômicos em geral e para previsões futebolísticas em particular, um padrão comumente utilizado em comparações desse tipo é a atribuição equiprovável de probabilidades $(P V=P E=P D=1 / 3)$, correspondente a um previsor que preguiçosamente atribui chances iguais a cada resultado em cada jogo. Para essa atribuição, a medida de DeFinetti é igual a

$$
(1 / 3-1)^{2}+2 \cdot(1 / 3-0)^{2}=0,6667 .
$$

Assim, podem ser considerados previsores (ou métodos de previsão) de qualidade minimamente aceitável aqueles que apresentarem medidas de DeFinetti menores que 0,6667 e podem ser considerados previsores (ou métodos de previsão) de má qualidade os que apresentarem medidas de DeFinetti superiores a 0,6667. 


\section{Capítulo 6 \\ Resultados}

\subsection{Características particulares de cada método}

Nos 390 jogos (64 da Copa do Mundo, 297 do Campeonato Brasileiro de 1998 e 29 do Torneio Rio-São Paulo de 1999) utilizados para comparação de métodos de previsão nesta tese e de suas respectivas atribuições de probabilidade (ver Apêndice A), pôde-se perceber algumas tendências e características qualitativas dos diversos métodos. O método "Chance" I, por exemplo, mostrou uma tendência à exacerbação do favoritismo, apresentando em muitos jogos, valores muito altos para a probabilidade de vitória do favorito. O "Chance" I ainda apresentou, por outro lado, tendência à "indecisão" em diversos jogos equilibrados, nos quais inexiste um favorito destacado à vitória. Para esses jogos, o método "Chance" I apresentou valores de probabilidades de vitória, empate e derrota bastante próximos de 1/3.

Outra característica observada em diversos jogos foi o antagonismo entre os métodos Implícito I e Implícito II e os explícitos (SD 0, SD I, "Chance" I e "Chance" II), no sentido de, em um mesmo jogo, atribuírem favoritismo a equipes distintas. Na Copa do Mundo de 1998, no jogo França x Brasil, por exemplo (ver Apêndice A), enquanto os métodos implícitos acompanharam a tendência popular dando favoritismo ao Brasil, os métodos explícitos apresentaram a vitória francesa como o resultado mais provável.

Os métodos "Chance" I e Implícito I foram os únicos a fornecerem previsões para todos os 390 jogos. Os demais métodos não garantem estimativas positivas para as esperanças marginais e não-negativas para a covariância. Por isso, diversas vezes esses métodos forneceram estimativas negativas para uma esperança marginal ou para a covariância, não sendo possível atribuir probabilidades aos resultados (ver Seção 3.6.2). A tabela abaixo mostra a quantidade de jogos em que cada método forneceu previsões. Nessa tabela, a Taxa de "funcionamento" é a freqüência amostral de estimativas válidas (em que tanto as esperanças marginais quanto a covariância foram não-negativas).

Nos casos em que os métodos SD 0, SD I ou "Chance II" forneceram estimativas negativas, não foram efetuadas "caminhadas" Quando há incoerência nos anúncios dos momentos (método Implícito II), a correção seria feita com outro tipo de "caminhada", por isso é importante lembrar a distinção entre esse problema e o problema das estimativas negativas de mínimos quadrados (SD 0, SD I, “Chance II").

\begin{tabular}{|l|c|c|}
\hline Método & $\mathbf{N}^{\mathbf{0}}$ de jogos & Taxa de "funcionamento" \\
\hline "Chance" I & 390 & $100,00 \%$ \\
\hline Implícito I & 390 & $100,00 \%$ \\
\hline SD 0 & 383 & $98,21 \%$ \\
\hline Implícito II & 361 & $92,56 \%$ \\
\hline "Chance" II & 297 & $76,15 \%$ \\
\hline SD I & 203 & $52,05 \%$ \\
\hline Implícito III ${ }^{\text {"I }}$ & 64 & $100,00 \%$ \\
\hline
\end{tabular}

O método Implícito III, aqui mencionado pela primeira vez, é exatamente igual ao Implícito I, diferindo apenas quanto à pessoa que anunciou as probabilidades. Esse método foi introduzido com o objetivo de investigar a sensibilidade do método Implícito I aos conhecimentos particulares de quem está efetuando as previsões. O método Implícito I se

${ }^{11}$ O Método Implícito III foi aplicado apenas nos 64 jogos da Copa do Mundo. 
baseou nos anúncios de uma pessoa que acompanha atentamente o futebol (o próprio aluno autor da tese), enquanto o Implícito III foi baseado em anúncios de uma pessoa que apresenta poucas condições de avaliar as equipes em confronto.

Na próxima Seção os métodos serão comparados em termos das medidas definidas no Capítulo 5 e da Taxa "de funcionamento". Tal atitude se justifica pelo fato de possuir serventia questionável um método com ótimas confiabilidade e exatidão, mas que freqüentemente exija o recurso da "caminhada" (Seção 3.6.2).

\subsection{Resultados das comparações dos métodos}

Os métodos de previsão de resultados de jogos de futebol (apresentados no Capítulo 3) foram comparados entre si (com relação aos 390 jogos mencionados na Seção 6.1) através das várias formas de verificação de previsões introduzidas no Capítulo 5. Também foi utilizada, como referência para a avaliação da qualidade ( $S k i l l)$ desses métodos, a medida de exatidão (Medida de DeFinetti) do método "Equiprovável".

O método Equiprovável", utilizado como parâmetro para a avaliação do atributo qualidade (vide Seção 5.6) dos métodos, consiste na atribuição preguiçosa de probabilidades iguais a $1 / 3$ aos três resultados possíveis (vitória, empate e derrota) em cada jogo.

Os valores das diversas medidas de verificação de probabilidades (definidas no Capítulo 5), calculados para cada um dos métodos, estão listados na tabela abaixo.

\begin{tabular}{|l||c|c|}
\hline Método & $\begin{array}{l}\text { Medida de } \\
\text { DeFinetti }\end{array}$ & $\begin{array}{l}\text { Medida de } \\
\text { Confiabilidade (MeC) }\end{array}$ \\
\hline "Chance" II & 0,6203 & 0,0052 \\
\hline "Chance" I & 0,6226 & 0,0061 \\
\hline SD 0 & 0,6247 & 0,0119 \\
\hline Implícito I & 0,6252 & 0,0024 \\
\hline Implícito II & 0,6322 & 0,0074 \\
\hline "Equiprovável" & 0,6667 & - \\
\hline SD I & 0,6850 & 0,0479 \\
\hline Implícito III & 0,8118 & 0,2014 \\
\hline
\end{tabular}

É importante perceber que a Medida de Confiabilidade do método "Equiprovável" é igual a zero (pois de todos os resultados que tiveram probabilidade anunciada $1 / 3$, exatamente 1/3 foi efetivamente observado), o que mostra que calibração (confiabilidade), por si só, não garante qualidade ao previsor.

Então, pode-se ordenar os métodos com relação a cada uma das medidas, fornecendo as classificações (em ordem decrescente de exatidão/confiabilidade) listadas na tabela abaixo. Nessa tabela, métodos com valores muito próximos entre si (como as Medidas de DeFinetti dos métodos Implícito I e Implícito II, por exemplo) estariam considerados "tecnicamente empatados", o que justifica as classificações como $1 / 2 / 3$, por exemplo (ver discussão no Capítulo 7).

\footnotetext{
${ }^{12}$ Soma de Distâncias quadráticas de DeFinetti dividida pelo número de jogos em que o método forneceu previsões.
} 


\begin{tabular}{|l|c|c|c|}
\hline \multirow{2}{*}{ Método } & \multicolumn{3}{|c|}{ Classificação segundo } \\
\cline { 2 - 4 } & $\begin{array}{l}\text { Medida de } \\
\text { DeFinetti }\end{array}$ & $\begin{array}{l}\text { Medida de } \\
\text { Confiabilidade }\end{array}$ & $\begin{array}{l}\text { Taxa de } \\
\text { "Funcionamento" }\end{array}$ \\
\hline Implícito I & $1 / 2 / 3 / 4$ & 1 & $1 / 2 / 3$ \\
\hline "Chance" I & $1 / 2 / 3 / 4$ & 3 & $1 / 2 / 3$ \\
\hline "Chance" II & $1 / 2 / 3 / 4$ & 2 & 6 \\
\hline SD 0 & $1 / 2 / 3 / 4$ & 5 & 4 \\
\hline Implícito II & 5 & 4 & 7 \\
\hline SD I & 6 & 6 & $1 / 2 / 3$ \\
\hline Implícito III & 7 & 7 & \\
\hline
\end{tabular}

Observando-se as tabelas acima, pode-se perceber que os métodos Implícito I, SD 0, "Chance" I, "Chance II" foram os que apresentaram os melhores desempenhos na verificação de atribuição de probabilidades. Embora o método SD 0 tenha apresentado uma fraca Medida de Confiabilidade, as Medidas de DeFinetti desses quatro métodos foram muito parecidas entre si.

Na tabela acima, os métodos foram ordenados de acordo com o que se poderia chamar de "comparação tríplice". Essa comparação consiste na simples verificação, para cada par de métodos, de qual supera o outro no maior número de critérios (dentre os três considerados). Assim, pode-se perceber que, nessa tabela, cada método é melhor ou igual do que todos os seguintes em pelo menos dois critérios.

Embora a natureza teórica desta tese permita destacar positivamente mais de um método, pode ser necessário, para aplicações práticas, identificar um único método como “o melhor de todos". Nesse caso, tal título seria concedido ao método Implícito I, que apresenta uma taxa de "funcionamento" de 100\% (conforme visto na Seção 6.1), é muito bem classificado (está entre os três primeiros lugares) segundo a Medida de Confiabilidade e, embora tenha ficado apenas em quarto lugar segundo a Medida de DeFinetti, foi o primeiro colocado no critério da "comparação tríplice".

É interessante perceber, na tabela acima, que o método Implícito II teve confiabilidade e exatidão inferiores ao método Implícito I. Da mesma forma, o método SD 0 superou, em ambos os critérios, o método SD I. Isso contraria a possível expectativa de que a estimação do parâmetro covariância (métodos SD I e Implícito II) enriquecesse as previsões em relação às produzidas sob a suposição de covariância nula (métodos SD 0 e Implícito I). Talvez, a consideração de um modelo mais rico conduza a uma maior dificuldade prática de obter coerência na expressão de parâmetros (Seção 3.5.2).

Por fim, cabe mencionar a ineficiência do método SD I, mal classificado em todas as formas de calibração, chegando até a apresentar uma Medida de DeFinetti de valor inferior (ainda que "tecnicamente empatada") à do método "Equiprovável".

Também se mostrou extremamente ineficiente o método Implícito III que foi o pior classificado tanto em confiabilidade quanto em exatidão, tendo apresentado uma Medida de DeFinetti bastante inferior à do método "Equiprovável". Essa derrota do método Implícito III (para o método "Equiprovável”) permitiria classificá-lo como um método de má qualidade, conforme definido na Seção 5.6. Entretanto, o método Implícito III não é um método diferente do Implícito I e a responsável pelos desempenhos díspares desses métodos é a diferença de qualidade das opiniões entre as pessoas que anunciaram as probabilidades. Assim, esse mau desempenho do método Implícito III, comparado ao do método Implícito I, ilustra de forma satisfatória a dependência sofrida por esse método à pessoa que anuncia as probabilidades. 
É importante perceber que os dois métodos mais bem classificados na "comparação tríplice" (e que tiveram Taxa de "funcionamento" igual a 100\%), "Chance" I e Implícito I têm índoles essencialmente antagônicas. O método "Chance" I está entre os explícitos e dentre estes é o único que, em seu processo de estimação de parâmetros, leva em consideração o fato de os gols seguirem uma distribuição de Poisson. Já sobre o método Implícito I, além do que o próprio nome diz (e dos comentários tecidos na Seção 3.6.4), pode-se reiterar a observação de que seu bom desempenho depende diretamente de quem seja o previsor. Concluindo, o excelente desempenho desses métodos mostra que as abordagens explícita e implícita possuem, ambas, muito boa eficácia.

Também é importante enfatizar que calibração (confiabilidade) e exatidão são importantes (como diz Murphy, 1967, 1972a, 1972b e 1977), caso contrário, o método "Equiprovável" que apresenta calibração perfeita poderia ser eleito "o melhor de todos". 


\section{Capítulo 7 \\ Considerações Finais}

Várias considerações metodológicas e até filosóficas foram tecidas ao longo desta tese sobre a oposição entre métodos explícitos (com espírito bem mais freqüentista), que exigem a construção de um banco de dados para efetuar suas previsões, e os métodos implícitos (com índole bayesiana), que permitem que se façam previsões até mesmo sem o conhecimento de qualquer resultado prévio.

Como foi visto no Capítulo 6, ambos apresentaram muito bom desempenho no que se refere à verificação de qualidade de suas previsões probabilísticas. Talvez a principal conclusão deste trabalho seja esta constatação de que os métodos Implícito I e "Chance" I (respectivamente, os métodos implícito e explícito de melhor comportamento) apresentaram desempenhos muito bons e praticamente iguais. Isso pode sugerir que uma abordagem bayesiana, quando adotada por uma pessoa com conhecimento do assunto a que se referem as previsões, possa até dispensar o uso de banco de dados, imprescindível em qualquer abordagem de índole mais freqüentista.

Também as medidas de verificação de qualidade de previsões abordadas no Capítulo 5 têm inspirações contrárias: a Medida de DeFinetti/Escore de Brier, de influência bayesiana, pode ser calculada individualmente até para um único jogo, enquanto a Medida de Confiabilidade (ou de calibração), de caráter freqüentista, só pode ser calculada após muitos jogos terem sido realizados e as freqüências relativas serem conhecidas.

Assim, nesse contexto de contrastes filosóficos, é importante (e curioso) perceber que o método ("freqüentista") "Chance" I foi o que apresentou o melhor desempenho em exatidão (Medida de DeFinetti, "bayesiana"), enquanto o método (bayesiano) Implícito I foi o mais bem calibrado (Medida de Confiabilidade, freqüentista).

Entretanto, é sempre importante lembrar que os bons resultados (medidas de exatidão e de confiabilidade) obtidos pelo método Implícito I dependem diretamente da pessoa que anunciou as probabilidades. Embora sua característica bayesiana permita que até um eremita que nunca em sua vida assistiu a uma partida de fatebol faça suas previsões, esse método tende a refletir, em suas medidas de exatidão e confiabilidade, a qualidade, habilidade e conhecimentos do previsor. Isso é ilustrado de forma convincente com o fraco desempenho do método Implícito III.

É importante considerar, contudo, que todos os resultados abordados e alcançados nesta tese (das probabilidades previstas às medidas de calibração) dependem fortemente dos critérios utilizados para a formação dos banco de dados (descritos no Capítulo 4) e dependem também de vários outros fatores, muitos deles essencialmente subjetivos:

- Escolha de jogos: quais competições devem e quais não devem ser consideradas na composição do banco de dados;

- Inclusão de times: restrição ou não aos jogos que envolvam um ou mais dos times participantes do campeonato, cujos jogos se quer prever;

- Escolha do sistema de pesos;

- Determinação da "idade máxima" dos jogos;

- A pessoa que anunciará as probabilidades (métodos Implícitos);

- Critérios de "empate técnico" para a "comparação tríplice", além do próprio critério de "comparação tríplice";

- Discretização escolhida para os valores de $p$ nas curvas de calibração;

- etc. 
Embora sejam apresentadas nos Capítulo 4 e 5 justificativas para critérios adotados, não há nada que os torne "os melhores possíveis" sob algum aspecto. Critérios diferentes dos utilizados nesta tese (porém igualmente válidos) eventualmente levarão a resultados diferentes dos aqui obtidos, o que não os invalida nem compromete o valor dos critérios desta tese. $\mathrm{O}$ sistema de pesos utilizado nesta tese, por exemplo, é bastante subjetivo, pois incorporou características (como a linearidade e o prazo de 8 anos para o "jubilamento" dos jogos) arbitrárias, sem qualquer justificativa teórica em que se possa apoiar. Um possível aprimoramento futuro deste trabalho consistiria na construção de um sistema de pesos com embasamento teórico (em vez da simples adaptação de sistemas alheios) buscando otimizá-lo dentro de uma determinada classe (de funções lineares, por exemplo).

Outra linha de pesquisa futura em que se pode investir é a expansão dos métodos tratados no Capítulo 3 para competições onde há mais de 2 participantes se enfrentando simultaneamente, como na Fórmula 1, corridas de cavalo, etc. e, mais geralmente, em aplicações a situações não-esportivas, onde, por exemplo, "jogo" é uma disputa entre vendedor e comprador de algum título no mercado financeiro, "gol" é unidade de lucro ou, em outro contexto, unidade de tempo obtida em sobrevida de pacientes, etc..

Também são possíveis linhas de pesquisa futura, a obtenção bayesiana de probabilidades através de atribuição de prioris próprias para o vetor $\left(\lambda_{1}, \lambda_{2}, \lambda_{12}\right)$; e o estudo de alternativas à solução apresentada na Seção 3.6.2 para o problema das "caminhadas" (como por exemplo a imposição de restrições no espaço de previsões nos modelos de mínimos quadrados). Também no campo das "caminhadas", pode-se estudar, exploratoriamente, a sensibilidade das probabilidades estimadas a perturbações no valor arbitrariamente escolhido para $\varepsilon$.

Pretende-se, também, construir testes formais de hipótese de boa calibração, removendo desse modo a arbitrariedade nas declarações de "empate técnico" entre métodos.

Sugere-se ainda como uma linha alternativa (e mais sofisticada) de pesquisa futura para exploração da "transitividade" e da quantidade de jogos dos arquivos de dados, o tratamento do problema de estimação dos parâmetros da distribuição "de Holgate" através da análise de redes neurais. 


\section{Capítulo 8 \\ Referências}

\subsection{Bibliografia}

ARRUDA, M. L. e WECHSLER, S. (2000), artigo-resposta a ser enviado para a revista Chance.

BARNETT, V. (1982), Comparative Statistical Inference, New York: Wiley.

BRIER, G. W. (1950), Verification of Forecasts Expressed in Terms of Probability, Monthly Weather Review 78, 1-3.

DAWID, A. P. (1982), The Well-Calibrated Bayesian, Journal of American Statistical Association 77 (379), 605-610.

DeFINETTI, B. (1963), La décision et les probabilités, Revue Roumaine des Mathématiques Pures et Apliquées VII, 3.

DeFINETTI, B. (1972), Probability, Induction and Statistics, London: John Wiley.

DWASS, M. e TEICHER, H. (1957), On Infinitely Divisible Random Vectors, Annals of Mathematical Statististics 28, 461-470.

FAHMEIR, L. e TUTZ, G. (1994), Multivariate Statistical Modelling Based on Generalized Linear Models, New York: Springer-Verlag.

GRIFFITHS, R.C., MILNE, R.K \& WOOD, R. (1979), Aspects of Correlation in Bivariate Poisson Distributions and Processes, Australian Journal of Statistics 21 (3), 238-255.

HAMDAN, M.A. e AL-BAYYATI, H.A. (1969), A Note on the Bivariate Poisson Distribution, The American Statistician 23 (4), 32-33.

HOLGATE, P. (1964), Estimation for the Bivariate Poisson Distribution, Biometrika 51, 241-245.

JOHNSON, M.E. e BROOKS, D.G. (1985), Can we Generate a Bivariate Poisson Distribution with a Negative Correlation? Statistics Group G-1 Report, Los Alamos National Laboratory.

JOHNSON, N.L, KOTZ, S. \& BALAKRISHNAN, N. (1997), Discrete Multivariate Distributions, New York: John Wiley \& Sons.

KADANE, J. B. \& LICHTENSTEIN, S. (1982), A Subjectivist View of Calibration, Technical Report \#233, Department of Statistics. Carnegie-Mellon University.

KELLER, J. B. (1994), A Characterization of the Poisson Distribution and the Probability of Winning a Game, The American Statistician 48 (4), 294-298.

KOCHERLAKOTA, S. \& KOCHERLAKOTA, K. (1992), Bivariate Discrete Distributions, New York: Marcel Dekker.

LEE, A. J. (1997), Modeling Scores in the Premier League: Is Manchester United Really the Best?, Chance 10 (1), 15-19.

LOSCHI, R. H. (1992), Coerência, Probabilidade e Calibração, Tese de Mestrado, IMEUSP.

LOUKAS, S., KEMP, C.D. e PAPAGEORGIOU, H. (1986), Even-point Estimation for the Bivariate Poisson Distribution, Biometrika 73, 222-223. 
MATTHEWS, R. (1999), Modelo Matemático Prevê Gols no Futebol, Folha de São Paulo, 22/03/1999, 1-12.

McCUlLAGH, P. e NELDER, J. A. (1989), Generalized Linear Models, New York: Chapman and Hall.

MURPHY, A. H. (1972a), Scalar and Vector Partitions of the Probability Score: Part I: Two-State Situation, Journal of Applied Meteorology 11, 273-282.

MURPHY, A. H. (1972b), Scalar and Vector Partitions of the Probability Score: Part II: N-State Situation, Journal of Applied Meteorology 11, 1183-1192.

MURPHY, A. H. e EPSTEIN, E. S. (1967), Verification of Probabilistic Predictions: A Brief Review, Journal of Applied Meteorology 6, 748-753.

MURPHY, A. H. e WINKLER, R. L. (1977), Reliability of Subjective Probability Forecasts of Precipitation and Temperature, Applied Statistics 26, 41-47.

PAPAGEORGIOU, H. e LOUKAS, S. (1988), Conditional Even Point Estimation for Bivariate Discrete Distributions, Communications in Statistics - Theory and Methods 17, 3403-3412.

PAULA, G. A. (1997), Modelos de Regressão, Apostila, IME-USP

ROBBINS, H. (1955), An Empirical Bayes Approach to Statistics, Proceedings of Third Berkeley Symposium on Mathematical Statistics and Probability 1, 151-161, Berkeley: University of California Press.

SEARLE, S. R. (1982), Matrix Algebra Useful for Statistics, New York: Wiley.

SEBER, G. A. F. (1977), Linear Regression Analysis, New York: Wiley.

SOARES, J. F. (1982), Chances de Vitória em uma Partida de Futebol, Atas do Sinape, 195-198

VENABLES, W. N. e RIPLEY, B. D. (1997), Modern Applied Statistics Using S-Plus, New York: Spriger-Verlag, 223-245.

\subsection{Sites da Internet}

Alex Zheglov's Independent International Soccer Team Ratings:

http://www.cs.rpi.edu/ zhegla/wsr/

Ch@nce de Gol

http://www.ime.usp.br/ mlarruda/chgol.html

FIFA: Federation Internationale de Football Association:

http://www.fifa.com/index.html

Ron Kessler's International Soccer Ratings:

http://www.sprynet.com/sprynet/ronkessler/rankings.htm

RSSSF: Rec.Sport.Soccer Statistics Foundation - Ranking Information:

http://www.risc.uni-linz.ac.at/non-official/rsssf/misc.htm\#rankinfo

WWRR: World Wide Ratings and Rankings:

http://www.com/wwrr/wwrr.htm 


\section{Apêndice A \\ Previsões Efetuadas pelos Métodos}

A.1. Jogos da Copa do Mundo de 1998
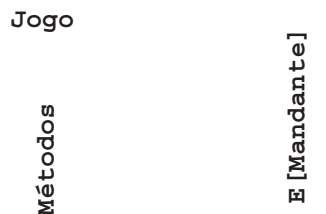

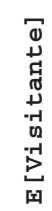

Brasil 2x1 Escócia

SD 0
SD I
"Chance" I
"Chance" II
Implícito I
Implícito II
Implícito III
Marrocos $2 \times 2$ Noruega

$$
\begin{array}{rrr}
1,997 & 1,997 & -0,163 \\
1,997 & 0,769 & -0,163 \\
1,304 & 1,304 & 0,149 \\
0,201 & 2,019 & -2,227 \\
1,5 & 1,5 & 0,5 \\
1,5 & 1,333 & 0,5 \\
3 & 3 & 0,2
\end{array}
$$

\begin{tabular}{|c|c|c|c|c|c|c|c|c|}
\hline SD 0 & 1,297 & 1,297 & 0,756 & 0,756 & 0 & $49,39 \%$ & $28,86 \%$ & $21,75 \%$ \\
\hline $\mathrm{SD} I$ & 1,297 & 1,901 & 0,756 & 1,36 & $-0,604$ & & & \\
\hline "Chance" I & 1,214 & 1,214 & 0,56 & 0,56 & 0 & $52,25 \%$ & $30,62 \%$ & $17,13 \%$ \\
\hline "Chance" II & $-0,946$ & 0,872 & $-1,524$ & 0,295 & $-1,819$ & & & \\
\hline Implícito I & 1,3 & 1,3 & 1,3 & 1,3 & 0 & $36,80 \%$ & $26,39 \%$ & $36,80 \%$ \\
\hline Implícito II & 1,3 & 0,597 & 1,3 & 0,597 & 0,703 & $28,95 \%$ & $42,10 \%$ & $28,95 \%$ \\
\hline Implícito III & 0,2 & 0,2 & 2 & 2 & 0 & $2,96 \%$ & $15,98 \%$ & $81,06 \%$ \\
\hline
\end{tabular}

$-0,163$

$-1,391$

0,149

$-0,408-1,819$

0,5

0,333

0,2
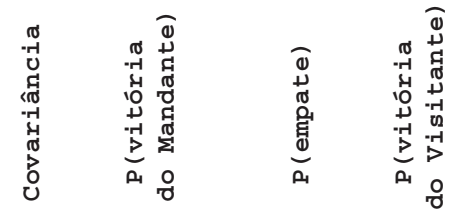

$\begin{array}{lrrrrrrrr}\text { SD 0 } & 1,314 & 1,314 & 3,587 & 3,587 & 0 & 9,58 \% & 11,32 \% & 78,98 \% \\ \text { SD I } & 1,314 & 3,546 & 3,587 & 5,819 & -2,232 & & & \\ \text { "Chance" I } & 0,794 & 0,794 & 2,785 & 2,785 & 0 & 7,97 \% & 13,34 \% & 78,68 \% \\ \text { "Chance" II } & -1,02 & 0,799 & 1,15 & 2,969 & -1,819 & & & \\ \text { Implícito I } & 1 & 1 & 1,5 & 1,5 & 0 & 25,22 \% & 25,98 \% & 48,79 \% \\ \text { Implícito II } & 1 & 0,54 & 1,5 & 1,04 & 0,46 & 18,87 \% & 33,89 \% & 47,24 \% \\ \text { Implícito III } & 1 & 1 & 3 & 3 & 0 & 9,39 \% & 13,11 \% & 77,47 \%\end{array}$

Itália 2x2 Chile

$\begin{array}{lrrrrrrrr}\text { SD 0 } & 1,228 & 1,228 & 0,981 & 0,981 & 0 & 41,80 \% & 28,77 \% & 29,43 \% \\ \text { SD I } & 1,228 & 0,816 & 0,981 & 0,569 & 0,412 & 38,39 \% & 38,09 \% & 23,52 \% \\ \text { "Chance" I } & 0,775 & 0,775 & 0,463 & 0,463 & 0 & 39,55 \% & 40,39 \% & 20,07 \% \\ \text { "Chance" II } & -1,176 & 0,643 & -1,321 & 0,498 & -1,819 & & & \\ \text { Implícito I } & 1,5 & 1,5 & 1,4 & 1,4 & 0 & 39,86 \% & 24,74 \% & 35,40 \% \\ \text { Implícito II } & 1,5 & 0,566 & 1,4 & 0,466 & 0,934 & 30,50 \% & 45,66 \% & 23,84 \% \\ \text { Implícito III } & 2 & 2 & 0,2 & 0,2 & 0 & 81,06 \% & 15,98 \% & 2,96 \%\end{array}$

Camarões 1x1 Áustria 
África do Sul 0x1 Dinamarca

$\begin{array}{lrrrrrrrr}\text { SD 0 } & 1,133 & 1,133 & 1,457 & 1,457 & 0 & 29,41 \% & 26,04 \% & 44,55 \% \\ \text { SD I } & 1,133 & 1,851 & 1,457 & 2,175 & -0,718 & & & \\ \text { "Chance" I } & 0,919 & 0,919 & 0,931 & 0,931 & 0 & 33,52 \% & 32,34 \% & 34,14 \% \\ \text { "Chance" II } & -1,048 & 0,771 & -0,842 & 0,977 & -1,819 & & & \\ \text { Implícito I } & 1 & 1 & 1,5 & 1,5 & 0 & 25,22 \% & 25,98 \% & 48,79 \% \\ \text { Implícito II } & 1 & 0,908 & 1,5 & 1,408 & 0,092 & 24,26 \% & 27,13 \% & 48,61 \% \\ \text { Implícito III } & 2 & 2 & 0,2 & 0,2 & 0 & 81,06 \% & 15,98 \% & 2,96 \%\end{array}$

Paraguai 0x0 Bulgária

$\begin{array}{lrrrrrrrr}\text { SD 0 } & 1,202 & 1,202 & 1,115 & 1,115 & 0 & 38,04 \% & 28,21 \% & 33,75 \% \\ \text { SD I } & 1,202 & 1,512 & 1,115 & 1,425 & -0,31 & & & \\ \text { "Chance" I } & 0,913 & 0,913 & 0,568 & 0,568 & 0 & 42,03 \% & 36,15 \% & 21,81 \% \\ \text { "Chance" II } & -0,86 & 0,959 & -1,411 & 0,408 & -1,819 & & & \\ \text { Implícito I } & 1,7 & 1,7 & 1,5 & 1,5 & 0 & 42,61 \% & 23,31 \% & 34,08 \% \\ \text { Implícito II } & 1,7 & 0,707 & 1,5 & 0,507 & 0,993 & 35,64 \% & 41,33 \% & 23,03 \% \\ \text { Implícito III } & 4 & 4 & 1 & 1 & 0 & 87,38 \% & 7,62 \% & 4,72 \%\end{array}$

França 3xo África do Sul

$\begin{array}{lrrrrrrrr}\text { SD 0 } & 2,07 & 2,07 & -0,283 & -0,283 & 0 & & & \\ \text { SD I } & 2,07 & 1,495 & -0,283 & -0,858 & 0,575 & & & \\ \text { "Chance" I } & 3,056 & 3,056 & 0,343 & 0,343 & 0 & 89,92 \% & 7,88 \% & 2,17 \% \\ \text { "Chance" II } & 0,516 & 2,335 & -1,914 & -0,095 & -1,819 & & & \\ \text { Implícito I } & 2 & 2 & 1,6 & 1,6 & 0 & 47,29 \% & 21,53 \% & 31,18 \% \\ \text { Implícito II } & 2 & 1,619 & 1,6 & 1,219 & 0,381 & 46,69 \% & 24,53 \% & 28,78 \% \\ \text { Implícito III } & 3 & 3 & 0,2 & 0,2 & 0 & 91,87 \% & 6,91 \% & 1,19 \%\end{array}$

Implícito III

Espanha 2×3 Nigéria

$\begin{array}{lrrrrrrrr}\text { SD 0 } & 2,34 & 2,34 & 0,599 & 0,599 & 0 & 76,48 \% & 15,75 \% & 7,76 \% \\ \text { SD I } & 2,34 & 3,256 & 0,599 & 1,515 & -0,916 & & & \\ \text { "Chance" I } & 1,628 & 1,628 & 0,333 & 0,333 & 0 & 70,09 \% & 22,80 \% & 7,11 \% \\ \text { "Chance" II } & -0,106 & 1,713 & -1,726 & 0,093 & -1,819 & & & \\ \text { Implícito I } & 1,7 & 1,7 & 1,4 & 1,4 & 0 & 44,69 \% & 23,57 \% & 31,74 \% \\ \text { Implícito II } & 1,7 & 1,596 & 1,4 & 1,296 & 0,104 & 44,43 \% & 24,51 \% & 31,06 \% \\ \text { Implícito III } & 1 & 1 & 3 & 3 & 0 & 9,39 \% & 13,11 \% & 77,47 \%\end{array}$

Implícito III

Holanda 0x0 Bélgica

$\begin{array}{lrrrrrrrr}\text { SD 0 } & 2,196 & 2,196 & 1,266 & 1,266 & 0 & 58,51 \% & 20,15 \% & 21,33 \% \\ \text { SD I } & 2,196 & 1,614 & 1,266 & 0,684 & 0,582 & 59,79 \% & 24,59 \% & 15,61 \% \\ \text { "Chance" I } & 1,54 & 1,54 & 0,781 & 0,781 & 0 & 55,37 \% & 25,68 \% & 18,95 \% \\ \text { "Chance" II } & -0,004 & 1,815 & -1,023 & 0,796 & -1,819 & & & \\ \text { Implícito I } & 1,5 & 1,5 & 1,1 & 1,1 & 0 & 46,42 \% & 25,77 \% & 27,81 \% \\ \text { Implícito II } & 1,5 & 1,203 & 1,1 & 0,803 & 0,297 & 45,41 \% & 29,95 \% & 24,64 \% \\ \text { Implícito III } & 1 & 1 & 0,2 & 0,2 & 0 & 56,21 \% & 36,45 \% & 7,33 \%\end{array}$


Coréia do Sul 1x3 México

$\begin{array}{lrrrrrrrr}\text { SD 0 } & 0,96 & 0,96 & 2,07 & 2,07 & 0 & 16,62 \% & 20,40 \% & 62,98 \% \\ \text { SD I } & 0,96 & 0,675 & 2,07 & 1,785 & 0,285 & 13,56 \% & 22,39 \% & 64,05 \% \\ \text { "Chance" I } & 0,655 & 0,655 & 1,714 & 1,714 & 0 & 13,84 \% & 23,21 \% & 62,95 \% \\ \text { "Chance" II } & -1,193 & 0,626 & -0,082 & 1,737 & -1,819 & & & \\ \text { Implícito I } & 1,4 & 1,4 & 1,5 & 1,5 & 0 & 35,40 \% & 24,74 \% & 39,86 \% \\ \text { Implícito II } & 1,4 & 0,54 & 1,5 & 0,64 & 0,86 & 25,66 \% & 42,29 \% & 32,04 \% \\ \text { Implícito III } & 1 & 1 & 2 & 2 & 0 & 18,26 \% & 21,17 \% & 60,57 \%\end{array}$

Iugoslávia 1x0 Irã

\begin{tabular}{|c|c|c|c|c|c|c|c|c|}
\hline SD 0 & 2,713 & 2,713 & 0,79 & 0,79 & 0 & $77,79 \%$ & $13,86 \%$ & $8,34 \%$ \\
\hline SD I & 2,713 & 1,294 & 0,79 & $-0,629$ & 1,419 & & & \\
\hline "Chance" I & 2,14 & 2,14 & 0,495 & 0,495 & 0 & $75,72 \%$ & $17,04 \%$ & $7,24 \%$ \\
\hline "Chance" II & 0,342 & 2,161 & $-1,456$ & 0,363 & $-1,819$ & & & \\
\hline Implícito I & 2 & 2 & 1 & 1 & 0 & $60,57 \%$ & $21,17 \%$ & $18,26 \%$ \\
\hline Implícito II & 2 & 1,408 & 1 & 0,408 & 0,592 & $62,35 \%$ & $27,04 \%$ & $10,60 \%$ \\
\hline Implícito III & 3 & 3 & 2 & 2 & 0 & $58,50 \%$ & $16,77 \%$ & $24,70 \%$ \\
\hline \multicolumn{9}{|c|}{ Inglaterra $2 \times 0$ Tunísia } \\
\hline SD 0 & 1,872 & 1,872 & 0,538 & 0,538 & 0 & $69,58 \%$ & $20,58 \%$ & $9,85 \%$ \\
\hline SD I & 1,872 & 1,427 & 0,538 & 0,093 & 0,445 & $72,85 \%$ & $24,87 \%$ & $2,27 \%$ \\
\hline "Chance" I & 1,566 & 1,566 & 0,179 & 0,179 & 0 & $73,39 \%$ & $22,71 \%$ & $3,90 \%$ \\
\hline "Chance" II & $-0,365$ & 1,454 & $-1,768$ & 0,051 & $-1,819$ & & & \\
\hline Implícito I & 2 & 2 & 1 & 1 & 0 & $60,57 \%$ & $21,17 \%$ & $18,26 \%$ \\
\hline Implícito II & 2 & 1,408 & 1 & 0,408 & 0,592 & $62,35 \%$ & $27,04 \%$ & $10,60 \%$ \\
\hline lícito III & 3 & 3 & 1 & 1 & 0 & $77,47 \%$ & $13,11 \%$ & $9,39 \%$ \\
\hline
\end{tabular}

Argentina 1x0 Japão

$\begin{array}{lrrrrrrrr}\text { SD 0 } & 1,901 & 1,901 & 0,324 & 0,324 & 0 & 75,97 \% & 18,56 \% & 5,47 \% \\ \text { SD I } & 1,901 & 1,798 & 0,324 & 0,221 & 0,103 & 76,94 \% & 19,10 \% & 3,95 \% \\ \text { "Chance" I } & 1,24 & 1,24 & 0,251 & 0,251 & 0 & 62,50 \% & 30,08 \% & 7,42 \% \\ \text { "Chance" II } & -0,415 & 1,404 & -1,978 & -0,159 & -1,819 & & & \\ \text { Implícito I } & 2,4 & 2,4 & 0,8 & 0,8 & 0 & 72,82 \% & 16,56 \% & 10,61 \% \\ \text { Implícito II } & 2,4 & -0,107 & 0,8 & -1,707 & 2,507 & & & \\ \text { Implícito III } & 0,2 & 0,2 & 2 & 2 & 0 & 2,96 \% & 15,98 \% & 81,06 \%\end{array}$

Jamaica 1x3 Croácia

$\begin{array}{lrrrrrrrr}\text { SD O } & 0,804 & 0,804 & 1,688 & 1,688 & 0 & 17,64 \% & 23,94 \% & 58,42 \% \\ \text { SD I } & 0,804 & -0,761 & 1,688 & 0,123 & 1,565 & & & \\ \text { "Chance" I } & 0,906 & 0,906 & 0,867 & 0,867 & 0 & 34,48 \% & 33,19 \% & 32,33 \% \\ \text { "Chance" II } & -1,354 & 0,465 & -0,694 & 1,125 & -1,819 & & & \\ \text { Implícito I } & 1,8 & 1,8 & 1,8 & 1,8 & 0 & 39,03 \% & 21,93 \% & 39,03 \% \\ \text { Implícito II } & 1,8 & 0,608 & 1,8 & 0,608 & 1,192 & 29,17 \% & 41,66 \% & 29,17 \% \\ \text { Implícito III } & 2 & 2 & 0,2 & 0,2 & 0 & 81,06 \% & 15,98 \% & 2,96 \%\end{array}$


Alemanha 2x0 EUA

$\begin{array}{lrrrrrrrr}\text { SD 0 } & 1,704 & 1,704 & 0,337 & 0,337 & 0 & 71,65 \% & 21,59 \% & 6,75 \% \\ \text { SD I } & 1,704 & 0,583 & 0,337 & -0,784 & 1,122 & & & \\ \text { "Chance" I } & 0,707 & 0,707 & 0,352 & 0,352 & 0 & 39,72 \% & 43,87 \% & 16,41 \% \\ \text { "Chance" II } & -0,742 & 1,077 & -1,892 & -0,073 & -1,819 & & & \\ \text { Implícito I } & 2,5 & 2,5 & 0,8 & 0,8 & 0 & 74,44 \% & 15,68 \% & 9,87 \% \\ \text { Implícito II } & 2,5 & 1,859 & 0,8 & 0,159 & 0,641 & 79,83 \% & 17,52 \% & 2,64 \% \\ \text { Implícito III } & 1 & 1 & 0,2 & 0,2 & 0 & 56,21 \% & 36,45 \% & 7,33 \%\end{array}$

Romênia 1x0 Colômbia

$\begin{array}{lrrrrrrrr}\text { SD 0 } & 1,857 & 1,857 & 1,397 & 1,397 & 0 & 48,38 \% & 22,59 \% & 29,03 \% \\ \text { SD I } & 1,857 & 1,604 & 1,397 & 1,144 & 0,253 & 48,03 \% & 24,79 \% & 27,18 \% \\ \text { "Chance" I } & 0,933 & 0,933 & 0,829 & 0,829 & 0 & 36,23 \% & 33,26 \% & 30,51 \% \\ \text { "Chance" II } & -0,606 & 1,213 & -0,984 & 0,835 & -1,819 & & & \\ \text { Implícito I } & 1,5 & 1,5 & 1,6 & 1,6 & 0 & 35,92 \% & 23,82 \% & 40,26 \% \\ \text { Implícito II } & 1,5 & 0,808 & 1,6 & 0,908 & 0,692 & 30,32 \% & 33,80 \% & 35,89 \%\end{array}$

Implícito III

$81,06 \% \quad 15,98 \% \quad 2,96 \%$

Brasil 3x0 Marrocos

$\begin{array}{lrrrrrrrr}\text { SD 0 } & 2,528 & 2,528 & 0,911 & 0,911 & 0 & 72,44 \% & 16,12 \% & 11,43 \% \\ \text { SD I } & 2,528 & 3,317 & 0,911 & 1,699 & -0,788 & & & \\ \text { "Chance" I } & 2,625 & 2,625 & 0,503 & 0,503 & 0 & 82,61 \% & 12,38 \% & 5,00 \% \\ \text { "Chance" II } & 0,622 & 2,44 & -1,109 & 0,71 & -1,819 & & & \\ \text { Implícito I } & 1,7 & 1,7 & 1 & 1 & 0 & 53,79 \% & 24,02 \% & 22,18 \% \\ \text { Implícito II } & 1,7 & 1,303 & 1 & 0,603 & 0,397 & 53,71 \% & 29,06 \% & 17,22 \% \\ \text { Implícito III } & 0,2 & 0,2 & 1 & 1 & 0 & 7,33 \% & 36,45 \% & 56,21 \%\end{array}$

Implícito III

Escócia 1x1 Noruega

$\begin{array}{lrrrrrrrr}\text { SD 0 } & 0,24 & 0,24 & 3,056 & 3,056 & 0 & 1,40 \% & 6,96 \% & 91,60 \% \\ \text { SD I } & 0,24 & -1,892 & 3,056 & 0,925 & 2,131 & & & \\ \text { "Chance" I } & 0,235 & 0,235 & 1,383 & 1,383 & 0 & 6,12 \% & 26,81 \% & 67,07 \% \\ \text { "Chance" II } & -2,138 & -0,319 & 0,729 & 2,548 & -1,819 & & & \\ \text { Implícito I } & 0,9 & 0,9 & 1,3 & 1,3 & 0 & 25,82 \% & 28,37 \% & 45,81 \% \\ \text { Implícito II } & 0,9 & 0,533 & 1,3 & 0,933 & 0,367 & 20,23 \% & 36,09 \% & 43,68 \% \\ \text { Implícito III } & 3 & 3 & 1 & 1 & 0 & 77,47 \% & 13,11 \% & 9,39 \%\end{array}$

ito III

Itália 3xo Camarões

$\begin{array}{lrrrrrrrr}\text { SD O } & 1,344 & 1,344 & 0,124 & 0,124 & 0 & 69,66 \% & 27,04 \% & 3,30 \% \\ \text { SD I } & 1,344 & 0,775 & 0,124 & -0,445 & 0,57 & & & \\ \text { "Chance" I } & 0,883 & 0,883 & 0,253 & 0,253 & 0 & 50,06 \% & 39,69 \% & 10,26 \% \\ \text { "Chance" II } & -0,929 & 0,89 & -2,082 & -0,263 & -1,819 & & & \\ \text { Implícito I } & 1,7 & 1,7 & 1,3 & 1,3 & 0 & 46,85 \% & 23,78 \% & 29,37 \% \\ \text { Implícito II } & 1,7 & 1,003 & 1,3 & 0,603 & 0,697 & 44,24 \% & 34,18 \% & 21,58 \% \\ \text { Implícito III } & 1 & 1 & 1 & 1 & 0 & 34,57 \% & 30,85 \% & 34,57 \%\end{array}$


Chile 1x1 Áustria

$\begin{array}{lrrrrrrrr}\text { SD 0 } & 2,153 & 2,153 & 0,639 & 0,639 & 0 & 72,38 \% & 17,95 \% & 9,67 \% \\ \text { SD I } & 2,153 & 3,345 & 0,639 & 1,831 & -1,192 & & & \\ \text { "Chance" I } & 2,223 & 2,223 & 0,491 & 0,491 & 0 & 77,18 \% & 16,10 \% & 6,72 \% \\ \text { "Chance" II } & -0,186 & 1,633 & -1,771 & 0,048 & -1,819 & & & \\ \text { Implícito I } & 1,7 & 1,7 & 1,5 & 1,5 & 0 & 42,61 \% & 23,31 \% & 34,08 \% \\ \text { Implícito II } & 1,7 & 0,803 & 1,5 & 0,603 & 0,897 & 37,04 \% & 37,91 \% & 25,05 \% \\ \text { Implícito III } & 2 & 2 & 1 & 1 & 0 & 60,57 \% & 21,17 \% & 18,26 \%\end{array}$

França 4x0 Arábia Saudita

$\begin{array}{lrrrrrrrr}\text { SD 0 } & 1,924 & 1,924 & -0,817 & -0,817 & 0 & & & \\ \text { SD I } & 1,924 & 1,589 & -0,817 & -1,152 & 0,335 & & & \\ \text { "Chance" I } & 2,327 & 2,327 & 0,208 & 0,208 & 0 & 85,46 \% & 12,25 \% & 2,29 \% \\ \text { "Chance" II } & 0,335 & 2,154 & -2,553 & -0,735 & -1,819 & & & \\ \text { Implícito I } & 1,9 & 1,9 & 0,7 & 0,7 & 0 & 65,90 \% & 21,12 \% & 12,98 \% \\ \text { Implícito II } & 1,9 & 1,603 & 0,7 & 0,403 & 0,297 & 67,44 \% & 23,66 \% & 8,90 \% \\ \text { Implícito III } & 0,2 & 0,2 & 2 & 2 & 0 & 2,96 \% & 15,98 \% & 81,06 \%\end{array}$

África do Sul 1xl Dinamarca

$\begin{array}{lrrrrrrrr}\text { SD 0 } & 1,133 & 1,133 & 1,457 & 1,457 & 0 & 29,41 \% & 26,04 \% & 44,55 \% \\ \text { SD I } & 1,133 & 1,851 & 1,457 & 2,175 & -0,718 & & & \\ \text { "Chance" I } & 0,919 & 0,919 & 0,931 & 0,931 & 0 & 33,52 \% & 32,34 \% & 34,14 \% \\ \text { "Chance" II } & -1,048 & 0,771 & -0,842 & 0,977 & -1,819 & & & \\ \text { Implícito I } & 1 & 1 & 1,5 & 1,5 & 0 & 25,22 \% & 25,98 \% & 48,79 \% \\ \text { Implícito II } & 1 & 0,908 & 1,5 & 1,408 & 0,092 & 24,26 \% & 27,13 \% & 48,61 \% \\ \text { Implícito III } & 2 & 2 & 0,2 & 0,2 & 0 & 81,06 \% & 15,98 \% & 2,96 \%\end{array}$

Implícito III

0,2

$81,06 \% \quad 15,98 \% \quad 2,96 \%$

Espanha 0x0 Paraguai

$\begin{array}{lrrrrrrrr}\text { SD 0 } & 1,801 & 1,801 & 0,589 & 0,589 & 0 & 66,70 \% & 21,79 \% & 11,51 \% \\ \text { SD I } & 1,801 & 2,468 & 0,589 & 1,257 & -0,668 & & & \\ \text { "Chance" I } & 1,231 & 1,231 & 0,344 & 0,344 & 0 & 59,26 \% & 30,43 \% & 10,31 \% \\ \text { "Chance" II } & -0,661 & 1,158 & -1,71 & 0,109 & -1,819 & & & \\ \text { Implícito I } & 1,8 & 1,8 & 1,4 & 1,4 & 0 & 47,02 \% & 22,95 \% & 30,03 \% \\ \text { Implícito II } & 1,8 & 1,126 & 1,4 & 0,726 & 0,674 & 45,02 \% & 31,40 \% & 23,58 \% \\ \text { Implícito III } & 3 & 3 & 0,2 & 0,2 & 0 & 91,87 \% & 6,91 \% & 1,19 \%\end{array}$

Nigéria 1xo Bulgária

$\begin{array}{lrrrrrrrr}\text { SD O } & 1,212 & 1,212 & 1,654 & 1,654 & 0 & 28,02 \% & 24,27 \% & 47,71 \% \\ \text { SD I } & 1,212 & 1,73 & 1,654 & 2,172 & -0,518 & & & \\ \text { "Chance" I } & 0,884 & 0,884 & 0,751 & 0,751 & 0 & 36,40 \% & 34,76 \% & 28,84 \% \\ \text { "Chance" II } & -0,876 & 0,943 & -0,856 & 0,963 & -1,819 & & & \\ \text { Implícito I } & 1,7 & 1,7 & 1,6 & 1,6 & 0 & 40,61 \% & 23,00 \% & 36,40 \% \\ \text { Implícito II } & 1,7 & 0,52 & 1,6 & 0,42 & 1,18 & 29,40 \% & 48,06 \% & 22,54 \% \\ \text { Implícito III } & 0,2 & 0,2 & 1 & 1 & 0 & 7,33 \% & 36,45 \% & 56,21 \%\end{array}$


Holanda 5xo Coréia do Sul

$\begin{array}{lrrrrrrrr}\text { SD 0 } & 2,99 & 2,99 & 0,403 & 0,403 & 0 & 88,36 \% & 8,81 \% & 2,81 \% \\ \text { SD I } & 2,99 & 2,719 & 0,403 & 0,132 & 0,271 & 90,95 \% & 8,05 \% & 0,97 \% \\ \text { "Chance" I } & 4,297 & 4,297 & 0,53 & 0,53 & 0 & 94,16 \% & 3,97 \% & 1,40 \% \\ \text { "Chance" II } & 1,283 & 3,102 & -1,386 & 0,433 & -1,819 & & & \\ \text { Implícito I } & 2,6 & 2,6 & 1 & 1 & 0 & 71,68 \% & 16,03 \% & 12,28 \% \\ \text { Implícito II } & 2,6 & 1,659 & 1 & 0,059 & 0,941 & 79,10 \% & 19,74 \% & 1,15 \% \\ \text { Implícito III } & 1 & 1 & 0,2 & 0,2 & 0 & 56,21 \% & 36,45 \% & 7,33 \%\end{array}$

Bélgica 2x2 México

$\begin{array}{lrrrrrrrr}\text { SD 0 } & 1,408 & 1,408 & 1,456 & 1,456 & 0 & 36,45 \% & 24,95 \% & 38,60 \% \\ \text { SD I } & 1,408 & 1,202 & 1,456 & 1,25 & 0,207 & 35,19 \% & 27,31 \% & 37,50 \% \\ \text { "Chance" I } & 0,965 & 0,965 & 1,104 & 1,104 & 0 & 31,36 \% & 30,13 \% & 38,51 \% \\ \text { "Chance" II } & -0,83 & 0,989 & -0,63 & 1,189 & -1,819 & & & \\ \text { Implícito I } & 1,8 & 1,8 & 1,7 & 1,7 & 0 & 40,92 \% & 22,25 \% & 36,82 \% \\ \text { Implícito II } & 1,8 & 0,603 & 1,7 & 0,503 & 1,197 & 31,30 \% & 43,93 \% & 24,77 \%\end{array}$

Implícito III

22

$1 \quad 0$

$60,57 \% \quad 21,17 \% \quad 18,26 \%$

Alemanha 2x2 Iugoslávia

$\begin{array}{lrrrrrrrr}\text { SD 0 } & 1,341 & 1,341 & 1,639 & 1,639 & 0 & 31,40 \% & 24,10 \% & 44,50 \% \\ \text { SD I } & 1,341 & -0,343 & 1,639 & -0,045 & 1,684 & & & \\ \text { "Chance" I } & 0,623 & 0,623 & 0,808 & 0,808 & 0 & 25,70 \% & 37,54 \% & 36,76 \% \\ \text { "Chance" II } & -0,952 & 0,867 & -0,516 & 1,303 & -1,819 & & & \\ \text { Implícito I } & 1,8 & 1,8 & 1,8 & 1,8 & 0 & 39,03 \% & 21,93 \% & 39,03 \% \\ \text { Implícito II } & 1,8 & 0,503 & 1,8 & 0,503 & 1,297 & 26,78 \% & 46,44 \% & 26,78 \% \\ \text { Implícito III } & 1 & 1 & 0,2 & 0,2 & 0 & 56,21 \% & 36,45 \% & 7,33 \%\end{array}$

Implícito III

EUA $1 \times 2$ Irã

\begin{tabular}{|c|c|c|c|c|c|c|c|c|}
\hline $\mathrm{SD} 0$ & 1,411 & 1,411 & 1,153 & 1,153 & 0 & $42,89 \%$ & $26,34 \%$ & $30,77 \%$ \\
\hline $\mathrm{SD} I$ & 1,411 & 0,063 & 1,153 & $-0,195$ & 1,348 & & & \\
\hline "Chance" I & 0,931 & 0,931 & 0,562 & 0,562 & 0 & $42,85 \%$ & $35,85 \%$ & $21,30 \%$ \\
\hline "Chance" II & $-1,033$ & 0,785 & $-1,245$ & 0,574 & $-1,819$ & & & \\
\hline Implícito I & 1,7 & 1,7 & 1,5 & 1,5 & 0 & $42,61 \%$ & $23,31 \%$ & $34,08 \%$ \\
\hline Implícito II & 1,7 & 0,803 & 1,5 & 0,603 & 0,897 & $37,04 \%$ & $37,91 \%$ & $25,05 \%$ \\
\hline Implícito III & 1 & 1 & 3 & 3 & 0 & $9,39 \%$ & $13,11 \%$ & $77,47 \%$ \\
\hline
\end{tabular}

Romênia 2x1 Inglaterra

\begin{tabular}{|c|c|c|c|c|c|c|c|c|}
\hline$S D 0$ & 1,437 & 1,437 & 1,381 & 1,381 & 0 & $38,68 \%$ & $25,18 \%$ & $36,15 \%$ \\
\hline $\mathrm{SD} I$ & 1,437 & 0,152 & 1,381 & 0,096 & 1,285 & $12,87 \%$ & $79,25 \%$ & $7,88 \%$ \\
\hline "Chance" I & 0,267 & 0,267 & 0,929 & 0,929 & 0 & $10,40 \%$ & $38,22 \%$ & $51,38 \%$ \\
\hline "Chance" II & $-1,014$ & 0,805 & $-0,91$ & 0,909 & $-1,819$ & & & \\
\hline Implícito I & 1,6 & 1,6 & 1,8 & 1,8 & 0 & $34,58 \%$ & $22,53 \%$ & $42,88 \%$ \\
\hline Implícito II & 1,6 & 0,859 & 1,8 & 1,059 & 0,741 & $28,98 \%$ & $31,41 \%$ & $39,61 \%$ \\
\hline Implícito III & 2 & 2 & 3 & 3 & 0 & $24,70 \%$ & $16,77 \%$ & $58,50 \%$ \\
\hline
\end{tabular}


Colômbia 1x0 Tunísia

$\begin{array}{lrrrrrrrr}\text { SD 0 } & 1,887 & 1,887 & 0,958 & 0,958 & 0 & 59,14 \% & 22,14 \% & 18,72 \% \\ \text { SD I } & 1,887 & 2,296 & 0,958 & 1,367 & -0,409 & & & \\ \text { "Chance" I } & 1,397 & 1,397 & 0,623 & 0,623 & 0 & 55,81 \% & 27,57 \% & 16,61 \% \\ \text { "Chance" II } & -0,439 & 1,38 & -1,359 & 0,46 & -1,819 & & & \\ \text { Implícito I } & 2 & 2 & 1 & 1 & 0 & 60,57 \% & 21,17 \% & 18,26 \% \\ \text { Implícito II } & 2 & 1,408 & 1 & 0,408 & 0,592 & 62,35 \% & 27,04 \% & 10,60 \% \\ \text { Implícito III } & 1 & 1 & 2 & 2 & 0 & 18,26 \% & 21,17 \% & 60,57 \%\end{array}$

Argentina 5x0 Jamaica

\begin{tabular}{|c|c|c|c|c|c|c|c|c|}
\hline SD 0 & 1,367 & 1,367 & $-0,231$ & $-0,231$ & 0 & & & \\
\hline SD I & 1,367 & 1,087 & $-0,231$ & $-0,511$ & 0,28 & & & \\
\hline "Chance" I & 0,864 & 0,864 & 0,151 & 0,151 & 0 & $52,59 \%$ & $41,11 \%$ & $6,30 \%$ \\
\hline "Chance" II & $-0,846$ & 0,973 & $-2,675$ & $-0,856$ & $-1,819$ & & & \\
\hline Implícito I & 2 & 2 & 1,2 & 1,2 & 0 & $55,91 \%$ & $21,55 \%$ & $22,54 \%$ \\
\hline Implícito II & 2 & 1,208 & 1,2 & 0,408 & 0,792 & $56,56 \%$ & $30,94 \%$ & $12,50 \%$ \\
\hline Implícito III & 3 & 3 & 0,2 & 0,2 & 0 & $91,87 \%$ & $6,91 \%$ & $1,19 \%$ \\
\hline Japão 0x1 Croácia & & & & & & & & \\
\hline $\mathrm{SD} 0$ & 1,358 & 1,358 & 2,221 & 2,221 & 0 & $22,86 \%$ & $20,13 \%$ & $57,01 \%$ \\
\hline SD I & 1,358 & 1,445 & 2,221 & 2,308 & $-0,087$ & & & \\
\hline "Chance" I & 1,501 & 1,501 & 1,244 & 1,244 & 0 & $43,20 \%$ & $25,33 \%$ & $31,47 \%$ \\
\hline "Chance" II & $-0,657$ & 1,161 & $-0,262$ & 1,556 & $-1,819$ & & & \\
\hline Implícito I & 0,9 & 0,9 & 2 & 2 & 0 & $16,16 \%$ & $20,86 \%$ & $62,98 \%$ \\
\hline Implícito II & 0,9 & 0,303 & 2 & 1,403 & 0,597 & $7,81 \%$ & $26,75 \%$ & $65,45 \%$ \\
\hline Implícito III & 0,2 & 0,2 & 1 & 1 & 0 & $7,33 \%$ & $36,45 \%$ & $56,21 \%$ \\
\hline
\end{tabular}

Brasil 1x2 Noruega

$\begin{array}{lrrrrrrrr}\text { SD 0 } & 2,072 & 2,072 & 2,729 & 2,729 & 0 & 29,49 \% & 18,02 \% & 52,47 \% \\ \text { SD I } & 2,072 & 2,987 & 2,729 & 3,644 & -0,915 & & & \\ \text { "Chance" I } & 1,588 & 1,588 & 1,067 & 1,067 & 0 & 49,44 \% & 25,04 \% & 25,52 \% \\ \text { "Chance" II } & 0,06 & 1,879 & 0,5 & 2,319 & -1,819 & & & \\ \text { Implícito I } & 1 & 1 & 1 & 1 & 0 & 34,57 \% & 30,85 \% & 34,57 \% \\ \text { Implícito II } & 1 & 0,715 & 1 & 0,715 & 0,285 & 31,09 \% & 37,83 \% & 31,09 \% \\ \text { Implícito III } & 3 & 3 & 1 & 1 & 0 & 77,47 \% & 13,11 \% & 9,39 \%\end{array}$

Escócia 0x3 Marrocos

\begin{tabular}{|c|c|c|c|c|c|c|c|c|}
\hline SD 0 & 0,695 & 0,695 & 1,238 & 1,238 & 0 & $20,85 \%$ & $29,87 \%$ & $49,27 \%$ \\
\hline SD I & 0,695 & 0,49 & 1,238 & 1,033 & 0,205 & $17,26 \%$ & $34,32 \%$ & $48,42 \%$ \\
\hline "Chance" I & 0,388 & 0,388 & 0,652 & 0,652 & 0 & $18,84 \%$ & $44,86 \%$ & $36,30 \%$ \\
\hline "Chance" II & $-1,577$ & 0,242 & $-0,88$ & 0,939 & $-1,819$ & & & \\
\hline Implícito I & 1 & 1 & 1,3 & 1,3 & 0 & $28,64 \%$ & $27,96 \%$ & $43,40 \%$ \\
\hline Implícito II & 1 & 0,666 & 1,3 & 0,966 & 0,334 & $24,37 \%$ & $34,30 \%$ & $41,33 \%$ \\
\hline Implícito III & 4 & 4 & 2 & 2 & 0 & $72,71 \%$ & $12,20 \%$ & $14,81 \%$ \\
\hline
\end{tabular}


Itália 2x1 Áustria

$\begin{array}{lrrrrrrrr}\text { SD 0 } & 1,734 & 1,734 & -0,027 & -0,027 & 0 & & & \\ \text { SD I } & 1,734 & 2,275 & -0,027 & 0,515 & -0,542 & & & \\ \text { "Chance" I } & 1,598 & 1,598 & 0,211 & 0,211 & 0 & 73,10 \% & 22,39 \% & 4,51 \% \\ \text { "Chance" II } & -0,637 & 1,181 & -2,367 & -0,549 & -1,819 & & & \\ \text { Implícito I } & 1,7 & 1,7 & 1,3 & 1,3 & 0 & 46,85 \% & 23,78 \% & 29,37 \% \\ \text { Implícito II } & 1,7 & 0,907 & 1,3 & 0,507 & 0,793 & 43,46 \% & 36,84 \% & 19,70 \% \\ \text { Implícito III } & 2 & 2 & 1 & 1 & 0 & 60,57 \% & 21,17 \% & 18,26 \%\end{array}$

Chile 1x1 Camarões

$\begin{array}{lrrrrrrrr}\text { SD 0 } & 1,763 & 1,763 & 0,79 & 0,79 & 0 & 60,55 \% & 23,04 \% & 16,41 \% \\ \text { SD I } & 1,763 & 1,585 & 0,79 & 0,613 & 0,177 & 61,04 \% & 24,80 \% & 14,15 \% \\ \text { "Chance" I } & 1,228 & 1,228 & 0,588 & 0,588 & 0 & 51,92 \% & 30,31 \% & 17,78 \% \\ \text { "Chance" II } & -0,478 & 1,341 & -1,485 & 0,334 & -1,819 & & & \\ \text { Implícito I } & 2 & 2 & 1,6 & 1,6 & 0 & 47,29 \% & 21,53 \% & 31,18 \% \\ \text { Implícito II } & 2 & 1,059 & 1,6 & 0,659 & 0,941 & 44,63 \% & 32,82 \% & 22,55 \% \\ \text { Implícito III } & 0,2 & 0,2 & 2 & 2 & 0 & 2,96 \% & 15,98 \% & 81,06 \%\end{array}$

França 2x1 Dinamarca

$\begin{array}{lrrrrrrrr}\text { SD 0 } & 1,619 & 1,619 & -0,41 & -0,41 & 0 & & & \\ \text { SD I } & 1,619 & 2,597 & -0,41 & 0,568 & -0,978 & & & \\ \text { "Chance" I } & 2,286 & 2,286 & 0,26 & 0,26 & 0 & 83,70 \% & 13,24 \% & 3,06 \% \\ \text { "Chance" II } & 0,011 & 1,83 & -2,213 & -0,394 & -1,819 & & & \\ \text { Implícito I } & 1,4 & 1,4 & 1,1 & 1,1 & 0 & 43,80 \% & 26,63 \% & 29,56 \% \\ \text { Implícito II } & 1,4 & 1,107 & 1,1 & 0,807 & 0,293 & 42,38 \% & 31,17 \% & 26,45 \% \\ \text { Implícito III } & 4 & 4 & 2 & 2 & 0 & 72,71 \% & 12,20 \% & 14,81 \%\end{array}$

Implícito III

África do Sul 2x2 Arábia Saudita

$\begin{array}{lrrrrrrrr}\text { SD 0 } & 1,439 & 1,439 & 1,051 & 1,051 & 0 & 45,98 \% & 26,45 \% & 27,57 \% \\ \text { SD I } & 1,439 & 0,705 & 1,051 & 0,317 & 0,734 & 40,61 \% & 44,49 \% & 14,90 \% \\ \text { "Chance" I } & 0,936 & 0,936 & 0,743 & 0,743 & 0 & 38,37 \% & 34,07 \% & 27,57 \% \\ \text { "Chance" II } & -0,724 & 1,095 & -1,183 & 0,636 & -1,819 & & & \\ \text { Implícito I } & 2 & 2 & 1,4 & 1,4 & 0 & 51,48 \% & 21,66 \% & 26,86 \% \\ \text { Implícito II } & 2 & 1,008 & 1,4 & 0,408 & 0,992 & 49,95 \% & 35,32 \% & 14,73 \% \\ \text { Implícito III } & 1 & 1 & 1 & 1 & 0 & 34,57 \% & 30,85 \% & 34,57 \%\end{array}$

Implícito III

Espanha 6x1 Bulgária

$\begin{array}{lrrrrrrrr}\text { SD 0 } & 1,712 & 1,712 & 0,414 & 0,414 & 0 & 69,61 \% & 22,01 \% & 8,38 \% \\ \text { SD I } & 1,712 & 2,199 & 0,414 & 0,901 & -0,487 & & & \\ \text { "Chance" I } & 1,381 & 1,381 & 0,24 & 0,24 & 0 & 66,86 \% & 26,88 \% & 6,26 \% \\ \text { "Chance" II } & -0,456 & 1,363 & -2,056 & -0,237 & -1,819 & & & \\ \text { Implícito I } & 1,7 & 1,7 & 1,3 & 1,3 & 0 & 46,85 \% & 23,78 \% & 29,37 \% \\ \text { Implícito II } & 1,7 & 1,003 & 1,3 & 0,603 & 0,697 & 44,24 \% & 34,18 \% & 21,58 \% \\ \text { Implícito III } & 2 & 2 & 1 & 1 & 0 & 60,57 \% & 21,17 \% & 18,26 \%\end{array}$


Nigéria 1×3 Paraguai

$\begin{array}{lrrrrrrrr}\text { SD 0 } & 1,3 & 1,3 & 1,829 & 1,829 & 0 & 27,27 \% & 22,87 \% & 49,86 \% \\ \text { SD I } & 1,3 & 2,193 & 1,829 & 2,722 & -0,893 & & & \\ \text { "Chance" I } & 0,788 & 0,788 & 1,076 & 1,076 & 0 & 26,41 \% & 31,71 \% & 41,88 \% \\ \text { "Chance" II } & -1,08 & 0,738 & -0,509 & 1,309 & -1,819 & & & \\ \text { Implícito I } & 1,3 & 1,3 & 1,4 & 1,4 & 0 & 34,81 \% & 25,78 \% & 39,41 \% \\ \text { Implícito II } & 1,3 & 1,008 & 1,4 & 1,108 & 0,292 & 32,56 \% & 29,77 \% & 37,67 \% \\ \text { Implícito III } & 0,2 & 0,2 & 4 & 4 & 0 & 0,48 \% & 2,96 \% & 96,28 \%\end{array}$

Holanda 2x2 México

$\begin{array}{lrrrrrrrr}\text { SD 0 } & 2,281 & 2,281 & 1,399 & 1,399 & 0 & 57,32 \% & 19,79 \% & 22,89 \% \\ \text { SD I } & 2,281 & 0,408 & 1,399 & -0,474 & 1,873 & & & \\ \text { "Chance" I } & 1,48 & 1,48 & 0,858 & 0,858 & 0 & 51,80 \% & 26,39 \% & 21,81 \% \\ \text { "Chance" II } & 0,105 & 1,923 & -0,715 & 1,104 & -1,819 & & & \\ \text { Implícito I } & 2,1 & 2,1 & 1 & 1 & 0 & 62,64 \% & 20,26 \% & 17,10 \% \\ \text { Implícito II } & 2,1 & 1,526 & 1 & 0,426 & 0,574 & 64,89 \% & 25,05 \% & 10,07 \% \\ \text { Implícito III } & 2 & 2 & 1 & 1 & 0 & 60,57 \% & 21,17 \% & 18,26 \%\end{array}$

Implícito III

Bélgica 1xl Coréia do Sul

$\begin{array}{lrrrrrrr}\text { SD 0 } & 1,937 & 1,937 & 0,875 & 0,875 & 0 & 62,26 \% & 21,41 \% \\ \text { SD I } & 1,937 & 3,085 & 0,875 & 2,023 & -1,148 & & \\ \text { "Chance" I } & 1,56 & 1,56 & 0,682 & 0,682 & 0 & 58,50 \% & 25,32 \% \\ \text { "Chance" II } & -0,39 & 1,428 & -1,301 & 0,518 & -1,819 & & \\ \text { Implícito I } & 1,5 & 1,5 & 1,3 & 1,3 & 0 & 41,96 \% & 25,14 \% \\ \text { Implícito II } & 1,5 & 1,108 & 1,3 & 0,908 & 0,392 & 39,97 \% & 30,49 \% \\ \text { Implícito III } & 2 & 2 & 1 & 1 & 0 & 60,57 \% & 21,17 \% \\ & & & & & 18,26 \%\end{array}$

Implícito III

Alemanha $2 \times 0$ Irã

$\begin{array}{lrrrrrrrr}\text { SD 0 } & 2,322 & 2,322 & 0,696 & 0,696 & 0 & 73,93 \% & 16,62 \% & 9,45 \% \\ \text { SD I } & 2,322 & 0,796 & 0,696 & -0,83 & 1,526 & & & \\ \text { "Chance" I } & 1,621 & 1,621 & 0,487 & 0,487 & 0 & 65,44 \% & 23,81 \% & 10,75 \% \\ \text { "Chance" II } & 0,052 & 1,871 & -1,31 & 0,509 & -1,819 & & & \\ \text { Implícito I } & 2,9 & 2,9 & 0,9 & 0,9 & 0 & 78,09 \% & 13,19 \% & 8,69 \% \\ \text { Implícito II } & 2,9 & 2,096 & 0,9 & 0,096 & 0,804 & 85,21 \% & 13,53 \% & 1,24 \% \\ \text { Implícito III } & 2 & 2 & 1 & 1 & 0 & 60,57 \% & 21,17 \% & 18,26 \%\end{array}$

EUA 0x1 Iugoslávia

$\begin{array}{lrrrrrrrr}\text { SD 0 } & 0,431 & 0,431 & 2,096 & 2,096 & 0 & 6,39 \% & 17,01 \% & 76,60 \% \\ \text { SD I } & 0,431 & -1,059 & 2,096 & 0,606 & 1,49 & & & \\ \text { "Chance" I } & 0,358 & 0,358 & 0,933 & 0,933 & 0 & 13,79 \% & 37,47 \% & 48,73 \% \\ \text { "Chance" II } & -2,037 & -0,219 & -0,451 & 1,367 & -1,819 & & & \\ \text { Implícito I } & 1 & 1 & 2 & 2 & 0 & 18,26 \% & 21,17 \% & 60,57 \% \\ \text { Implícito II } & 1 & 0,526 & 2 & 1,526 & 0,474 & 12,59 \% & 25,43 \% & 61,98 \% \\ \text { Implícito III } & 4 & 4 & 0,2 & 0,2 & 0 & 96,28 \% & 2,96 \% & 0,48 \%\end{array}$


Romênia 1x1 Tunísia

$\begin{array}{lrrrrrrrr}\text { SD 0 } & 2,585 & 2,585 & 1,195 & 1,195 & 0 & 67,29 \% & 17,06 \% & 15,65 \% \\ \text { SD I } & 2,585 & 3,119 & 1,195 & 1,729 & -0,534 & & & \\ \text { "Chance" I } & 2,067 & 2,067 & 0,82 & 0,82 & 0 & 66,31 \% & 19,87 \% & 13,83 \% \\ \text { "Chance" II } & 0,311 & 2,13 & -0,988 & 0,831 & -1,819 & & & \\ \text { Implícito I } & 1,9 & 1,9 & 1,2 & 1,2 & 0 & 53,72 \% & 22,35 \% & 23,94 \% \\ \text { Implícito II } & 1,9 & 1,103 & 1,2 & 0,403 & 0,797 & 53,35 \% & 33,20 \% & 13,45 \% \\ \text { Implícito III } & 4 & 4 & 2 & 2 & 0 & 72,71 \% & 12,20 \% & 14,81 \%\end{array}$

Colômbia 0x2 Inglaterra

\begin{tabular}{|c|c|c|c|c|c|c|c|c|}
\hline $\mathrm{SD} 0$ & 0,74 & 0,74 & 1,144 & 1,144 & 0 & $23,72 \%$ & $31,06 \%$ & $45,22 \%$ \\
\hline $\mathrm{SD} I$ & 0,74 & 0,229 & 1,144 & 0,633 & 0,511 & $11,62 \%$ & $48,61 \%$ & $39,77 \%$ \\
\hline "Chance" I & 0,181 & 0,181 & 0,707 & 0,707 & 0 & $8,66 \%$ & $46,61 \%$ & $44,73 \%$ \\
\hline "Chance" II & $-1,764$ & 0,055 & $-1,281$ & 0,537 & $-1,819$ & & & \\
\hline Implícito I & 1,3 & 1,3 & 1,5 & 1,5 & 0 & $32,90 \%$ & $25,14 \%$ & $41,96 \%$ \\
\hline Implícito II & 1,3 & 0,908 & 1,5 & 1,108 & 0,392 & $29,54 \%$ & $30,49 \%$ & $39,97 \%$ \\
\hline Implícito III & 0,2 & 0,2 & 1 & 1 & 0 & $7,33 \%$ & $36,45 \%$ & $56,21 \%$ \\
\hline
\end{tabular}

Argentina 1x0 Croácia

\begin{tabular}{|c|c|c|c|c|c|c|c|c|}
\hline $\mathrm{SD} 0$ & 2,11 & 2,11 & 1,396 & 1,396 & 0 & $53,91 \%$ & $20,93 \%$ & $25,16 \%$ \\
\hline SD I & 2,11 & 2,097 & 1,396 & 1,383 & 0,013 & $53,92 \%$ & $21,00 \%$ & $25,08 \%$ \\
\hline "Chance" I & 2,246 & 2,246 & 0,376 & 0,376 & 0 & $80,35 \%$ & $14,83 \%$ & $4,82 \%$ \\
\hline "Chance" II & $-0,084$ & 1,735 & $-1,253$ & 0,566 & $-1,819$ & & & \\
\hline Implícito I & 1,8 & 1,8 & 1,6 & 1,6 & 0 & $42,88 \%$ & $22,53 \%$ & $34,58 \%$ \\
\hline Implícito II & 1,8 & 0,703 & 1,6 & 0,503 & 1,097 & $35,57 \%$ & $41,52 \%$ & $22,92 \%$ \\
\hline Implícito III & 1 & 1 & 0,2 & 0,2 & 0 & $56,21 \%$ & $36,45 \%$ & $7,33 \%$ \\
\hline
\end{tabular}

Implícito III

Japão 1x2 Jamaica

\begin{tabular}{|c|c|c|c|c|c|c|c|c|}
\hline SD 0 & 0,615 & 0,615 & 0,595 & 0,595 & 0 & $29,75 \%$ & $41,77 \%$ & $28,48 \%$ \\
\hline SD I & 0,615 & 0,261 & 0,595 & 0,241 & 0,354 & $18,62 \%$ & $64,35 \%$ & $17,02 \%$ \\
\hline "Chance" I & 0,578 & 0,578 & 0,5 & 0,5 & 0 & $30,24 \%$ & $44,60 \%$ & $25,15 \%$ \\
\hline "Chance" II & $-1,419$ & 0,4 & $-1,685$ & 0,134 & $-1,819$ & & & \\
\hline Implícito I & 1 & 1 & 1,5 & 1,5 & 0 & $25,22 \%$ & $25,98 \%$ & $48,79 \%$ \\
\hline Implícito II & 1 & 0,54 & 1,5 & 1,04 & 0,46 & $18,87 \%$ & $33,89 \%$ & $47,24 \%$ \\
\hline Implícito III & 1 & 1 & 0,2 & 0,2 & 0 & $56,21 \%$ & $36,45 \%$ & $7,33 \%$ \\
\hline
\end{tabular}

Itália 1x0 Noruega

\begin{tabular}{|c|c|c|c|c|c|c|c|c|}
\hline SD 0 & 1,252 & 1,252 & 2,38 & 2,38 & 0 & $18,84 \%$ & $18,76 \%$ & $62,40 \%$ \\
\hline SD I & 1,252 & 0,981 & 2,38 & 2,108 & 0,272 & $16,61 \%$ & $20,11 \%$ & $63,27 \%$ \\
\hline "Chance" I & 0,883 & 0,883 & 1,404 & 1,404 & 0 & $23,63 \%$ & $27,23 \%$ & $49,14 \%$ \\
\hline "Chance" II & $-1,082$ & 0,843 & 0,181 & 2,105 & $-1,924$ & & & \\
\hline Implícito I & 1,4 & 1,4 & 1,4 & 1,4 & 0 & $37,36 \%$ & $25,28 \%$ & $37,36 \%$ \\
\hline Implícito II & 1,4 & 0,64 & 1,4 & 0,64 & 0,76 & $29,80 \%$ & $40,40 \%$ & $29,80 \%$ \\
\hline Implícito III & 2 & 2 & 1 & 1 & 0 & $60,57 \%$ & $21,17 \%$ & $18,26 \%$ \\
\hline
\end{tabular}


Brasil 4xl Chile

$\begin{array}{lrrrrrrrr}\text { SD 0 } & 2,242 & 2,242 & 1,027 & 1,027 & 0 & 64,81 \% & 19,10 \% & 16,09 \% \\ \text { SD I } & 2,242 & 2,738 & 1,027 & 1,523 & -0,496 & & & \\ \text { "Chance" I } & 2,192 & 2,192 & 0,587 & 0,587 & 0 & 74,35 \% & 17,16 \% & 8,48 \% \\ \text { "Chance" II } & 0,274 & 2,199 & -1,169 & 0,755 & -1,924 & & & \\ \text { Implícito I } & 2 & 2 & 1,1 & 1,1 & 0 & 58,21 \% & 21,40 \% & 20,39 \% \\ \text { Implícito II } & 2 & 1,426 & 1,1 & 0,526 & 0,574 & 59,36 \% & 27,02 \% & 13,61 \% \\ \text { Implícito III } & 2 & 2 & 1 & 1 & 0 & 60,57 \% & 21,17 \% & 18,26 \%\end{array}$

França 0x0 Paraguai ${ }^{13}$

\begin{tabular}{|c|c|c|c|c|c|c|c|c|}
\hline SD 0 & 1,707 & 1,707 & 0,157 & 0,157 & 0 & $77,06 \%$ & $19,95 \%$ & $3,00 \%$ \\
\hline SD I & 1,707 & 1,724 & 0,157 & 0,174 & $-0,017$ & & & \\
\hline "Chance" I & 1,663 & 1,663 & 0,376 & 0,376 & 0 & $69,61 \%$ & $22,52 \%$ & $7,87 \%$ \\
\hline "Chance" II & $-0,195$ & 1,729 & $-1,79$ & 0,134 & $-1,924$ & & & \\
\hline Implícito I & 1,4 & 1,4 & 0,9 & 0,9 & 0 & $48,59 \%$ & $27,24 \%$ & $24,17 \%$ \\
\hline Implícito II & 1,4 & 1,403 & 0,9 & 0,903 & $-0,003$ & & & \\
\hline Implícito III & 4 & 4 & 1 & 1 & 0 & $87,38 \%$ & $7,62 \%$ & $4,72 \%$ \\
\hline \multicolumn{9}{|c|}{ Dinamarca $4 \times 1$ Nigéria } \\
\hline SD 0 & 1,434 & 1,434 & 1,423 & 1,423 & 0 & $37,75 \%$ & $24,99 \%$ & $37,26 \%$ \\
\hline SD I & 1,434 & 2,683 & 1,423 & 2,672 & $-1,249$ & & & \\
\hline "Chance" I & 0,929 & 0,929 & 1,125 & 1,125 & 0 & $29,86 \%$ & $30,16 \%$ & $39,97 \%$ \\
\hline "Chance" II & $-0,816$ & 1,109 & $-0,807$ & 1,117 & $-1,924$ & & & \\
\hline Implícito I & 0,8 & 0,8 & 1,8 & 1,8 & 0 & $16,22 \%$ & $22,65 \%$ & $61,13 \%$ \\
\hline Implícito II & 0,8 & 0,859 & 1,8 & 1,859 & $-0,059$ & & & \\
\hline mplícito III & 4 & 4 & 1 & 1 & 0 & $87,38 \%$ & $7,62 \%$ & $4,72 \%$ \\
\hline
\end{tabular}

Alemanha 2x1 México

$\begin{array}{lrrrrrrrr}\text { SD 0 } & 1,943 & 1,943 & 0,922 & 0,922 & 0 & 61,24 \% & 21,50 \% & 17,26 \% \\ \text { SD I } & 1,943 & 0,98 & 0,922 & -0,04 & 0,962 & & & \\ \text { "Chance" I } & 1,439 & 1,439 & 0,628 & 0,628 & 0 & 56,82 \% & 26,95 \% & 16,23 \% \\ \text { "Chance" II } & -0,188 & 1,736 & -1,044 & 0,88 & -1,924 & & & \\ \text { Implícito I } & 1,9 & 1,9 & 1,6 & 1,6 & 0 & 45,11 \% & 22,04 \% & 32,85 \% \\ \text { Implícito II } & 1,9 & 1,619 & 1,6 & 1,319 & 0,281 & 44,49 \% & 24,29 \% & 31,22 \% \\ \text { Implícito III } & 4 & 4 & 1 & 1 & 0 & 87,38 \% & 7,62 \% & 4,72 \%\end{array}$

Iugoslávia 1x2 Holanda

$\begin{array}{lrrrrrrrr}\text { SD 0 } & 1,806 & 1,806 & 1,694 & 1,694 & 0 & 41,17 \% & 22,25 \% & 36,58 \% \\ \text { SD I } & 1,806 & 0,039 & 1,694 & -0,073 & 1,767 & & & \\ \text { "Chance" I } & 1,139 & 1,139 & 0,92 & 0,92 & 0 & 40,61 \% & 30,06 \% & 29,33 \% \\ \text { "Chance" II } & -0,461 & 1,463 & -0,524 & 1,4 & -1,924 & & & \\ \text { Implícito I } & 1,4 & 1,4 & 1,4 & 1,4 & 0 & 37,36 \% & 25,28 \% & 37,36 \% \\ \text { Implícito II } & 1,4 & 0,807 & 1,4 & 0,807 & 0,593 & 32,43 \% & 35,14 \% & 32,43 \% \\ \text { Implícito III } & 2 & 2 & 3 & 3 & 0 & 24,70 \% & 16,77 \% & 58,50 \%\end{array}$

${ }^{13}$ Todas as previsões se referem unicamente ao resultado dos 90 minutos regulamentares. 
Argentina $2 \times 2$ Inglaterra

\begin{tabular}{|c|c|c|c|c|c|c|c|c|}
\hline SD 0 & 0,994 & 0,994 & 0,761 & 0,761 & 0 & $39,88 \%$ & $33,06 \%$ & $27,06 \%$ \\
\hline SD I & 0,994 & 0,903 & 0,761 & 0,67 & 0,091 & $39,03 \%$ & $35,32 \%$ & $25,65 \%$ \\
\hline "Chance" I & 0,35 & 0,35 & 0,339 & 0,339 & 0 & $22,21 \%$ & $56,37 \%$ & $21,42 \%$ \\
\hline "Chance" II & $-1,323$ & 0,601 & $-1,685$ & 0,239 & $-1,924$ & & & \\
\hline Implícito I & 1,6 & 1,6 & 1,6 & 1,6 & 0 & $38,29 \%$ & $23,43 \%$ & $38,29 \%$ \\
\hline Implícito II & 1,6 & 0,44 & 1,6 & 0,44 & 1,16 & $25,05 \%$ & $49,89 \%$ & $25,05 \%$ \\
\hline Implícito III & 0,2 & 0,2 & 1 & 1 & 0 & $7,33 \%$ & $36,45 \%$ & $56,21 \%$ \\
\hline
\end{tabular}

Croácia $1 \times 0$ Romênia

$\begin{array}{lrrrrrrrr}\text { SD 0 } & 1,773 & 1,773 & 2,158 & 2,158 & 0 & 32,27 \% & 20,56 \% & 47,17 \% \\ \text { SD I } & 1,773 & 2,347 & 2,158 & 2,732 & -0,574 & & & \\ \text { "Chance" I } & 1,252 & 1,252 & 2,076 & 2,076 & 0 & 22,61 \% & 21,03 \% & 56,36 \% \\ \text { "Chance" II } & -0,443 & 1,482 & -0,013 & 1,911 & -1,924 & & & \\ \text { Implícito I } & 1,4 & 1,4 & 1,5 & 1,5 & 0 & 35,40 \% & 24,74 \% & 39,86 \% \\ \text { Implícito II } & 1,4 & 0,397 & 1,5 & 0,497 & 1,003 & 21,83 \% & 49,37 \% & 28,79 \% \\ \text { Implícito III } & 0,2 & 0,2 & 1 & 1 & 0 & 7,33 \% & 36,45 \% & 56,21 \%\end{array}$

França 0xo Itália

\begin{tabular}{|c|c|c|c|c|c|c|c|c|}
\hline SD 0 & 1,35 & 1,35 & 0,346 & 0,346 & 0 & $62,65 \%$ & $27,96 \%$ & $9,38 \%$ \\
\hline$S D I$ & 1,35 & 1,105 & 0,346 & 0,101 & 0,245 & $63,26 \%$ & $33,36 \%$ & $3,37 \%$ \\
\hline "Chance" I & 1,216 & 1,216 & 0,435 & 0,435 & 0 & $56,00 \%$ & $30,77 \%$ & $13,24 \%$ \\
\hline "Chance" II & $-0,328$ & 1,596 & $-1,658$ & 0,266 & $-1,924$ & & & \\
\hline Implícito I & 1,4 & 1,4 & 1,3 & 1,3 & 0 & $39,41 \%$ & $25,78 \%$ & $34,81 \%$ \\
\hline Implícito II & 1,4 & 0,907 & 1,3 & 0,807 & 0,493 & $35,88 \%$ & $33,81 \%$ & $30,31 \%$ \\
\hline Implícito III & 1 & 1 & 3 & 3 & 0 & $9,39 \%$ & $13,11 \%$ & $77,47 \%$ \\
\hline
\end{tabular}

Implícito III

Brasil 3x2 Dinamarca

$\begin{array}{lrrrrrrrr}\text { SD 0 } & 2,178 & 2,178 & 0,42 & 0,42 & 0 & 78,22 \% & 15,97 \% & 5,80 \% \\ \text { SD I } & 2,178 & 2,848 & 0,42 & 1,09 & -0,67 & & & \\ \text { "Chance" I } & 2,025 & 2,025 & 0,391 & 0,391 & 0 & 76,40 \% & 17,53 \% & 6,07 \% \\ \text { "Chance" II } & 0,154 & 2,079 & -1,66 & 0,265 & -1,924 & & & \\ \text { Implícito I } & 2,5 & 2,5 & 1,6 & 1,6 & 0 & 57,36 \% & 18,73 \% & 23,90 \% \\ \text { Implícito II } & 2,5 & 1,059 & 1,6 & 0,159 & 1,441 & 59,69 \% & 34,77 \% & 5,54 \% \\ \text { Implícito III } & 3 & 3 & 0,2 & 0,2 & 0 & 91,87 \% & 6,91 \% & 1,19 \%\end{array}$

Holanda 2x1 Argentina

\begin{tabular}{|c|c|c|c|c|c|c|c|c|}
\hline SD 0 & 1,67 & 1,67 & 1,352 & 1,352 & 0 & $45,01 \%$ & $23,87 \%$ & $31,12 \%$ \\
\hline SD I & 1,67 & 0,196 & 1,352 & $-0,122$ & 1,474 & & & \\
\hline "Chance" I & 0,547 & 0,547 & 0,825 & 0,825 & 0 & $22,52 \%$ & $38,16 \%$ & $39,32 \%$ \\
\hline "Chance" II & $-0,715$ & 1,21 & $-0,901$ & 1,024 & $-1,924$ & & & \\
\hline Implícito I & 2 & 2 & 2 & 2 & 0 & $39,65 \%$ & $20,70 \%$ & $39,65 \%$ \\
\hline Implícito II & 2 & 1,507 & 2 & 1,507 & 0,493 & $37,88 \%$ & $24,24 \%$ & $37,88 \%$ \\
\hline Implícito III & 1 & 1 & 3 & 3 & 0 & $9,39 \%$ & $13,11 \%$ & $77,47 \%$ \\
\hline
\end{tabular}


Alemanha 0x3 Croácia

\begin{tabular}{|c|c|c|c|c|c|c|c|c|}
\hline SD 0 & 2,209 & 2,209 & 1,168 & 1,168 & 0 & $60,96 \%$ & $19,83 \%$ & $19,21 \%$ \\
\hline SD I & 2,209 & 2,054 & 1,168 & 1,013 & 0,155 & $61,39 \%$ & $20,71 \%$ & $17,89 \%$ \\
\hline "Chance" I & 2,228 & 2,228 & 0,667 & 0,667 & 0 & $73,01 \%$ & $17,36 \%$ & $9,63 \%$ \\
\hline "Chance" II & 0,126 & 2,05 & $-0,949$ & 0,976 & $-1,924$ & & & \\
\hline Implícito I & 1,9 & 1,9 & 1,3 & 1,3 & 0 & $51,47 \%$ & $22,36 \%$ & $26,17 \%$ \\
\hline Implícito II & 1,9 & 1,003 & 1,3 & 0,403 & 0,897 & $49,92 \%$ & $35,48 \%$ & $14,60 \%$ \\
\hline Implícito III & 2 & 2 & 1 & 1 & 0 & $60,57 \%$ & $21,17 \%$ & $18,26 \%$ \\
\hline
\end{tabular}

Brasil 1x1 Holanda

\begin{tabular}{|c|c|c|c|c|c|c|c|c|}
\hline SD 0 & 1,905 & 1,905 & 1,683 & 1,683 & 0 & $43,57 \%$ & $21,85 \%$ & $34,58 \%$ \\
\hline $\mathrm{SD} I$ & 1,905 & 0,805 & 1,683 & 0,583 & 1,1 & $37,63 \%$ & $38,10 \%$ & $24,27 \%$ \\
\hline "Chance" I & 1,459 & 1,459 & 0,884 & 0,884 & 0 & $50,57 \%$ & $26,60 \%$ & $22,82 \%$ \\
\hline "Chance" II & $-0,059$ & 1,865 & $-0,342$ & 1,583 & $-1,924$ & & & \\
\hline Implícito I & 2 & 2 & 2 & 2 & 0 & $39,65 \%$ & $20,70 \%$ & $39,65 \%$ \\
\hline Implícito II & 2 & 0,813 & 2 & 0,813 & 1,187 & $32,51 \%$ & $34,98 \%$ & $32,51 \%$ \\
\hline
\end{tabular}

Implícito III

França 2x1 Croácia

$\begin{array}{lrrrrrrrr}\text { SD 0 } & 2,153 & 2,153 & 0,734 & 0,734 & 0 & 70,05 \% & 18,54 \% & 11,41 \% \\ \text { SD I } & 2,153 & 1,735 & 0,734 & 0,316 & 0,418 & 72,92 \% & 20,94 \% & 6,14 \% \\ \text { "Chance" I } & 3,156 & 3,156 & 0,52 & 0,52 & 0 & 87,81 \% & 8,74 \% & 3,41 \% \\ \text { "Chance" II } & 0,442 & 2,367 & -1,253 & 0,671 & -1,924 & & & \\ \text { Implícito I } & 1,5 & 1,5 & 1,4 & 1,4 & 0 & 39,86 \% & 24,74 \% & 35,40 \% \\ \text { Implícito II } & 1,5 & 0,903 & 1,4 & 0,803 & 0,597 & 35,83 \% & 33,92 \% & 30,25 \% \\ \text { Implícito III } & 1 & 1 & 0,2 & 0,2 & 0 & 56,21 \% & 36,45 \% & 7,33 \%\end{array}$

Holanda 1x2 Croácia

\begin{tabular}{|c|c|c|c|c|c|c|c|c|}
\hline SD 0 & 2,552 & 2,552 & 1,543 & 1,543 & 0 & $59,48 \%$ & $18,32 \%$ & $22,19 \%$ \\
\hline SD I & 2,552 & 2,034 & 1,543 & 1,025 & 0,518 & $60,69 \%$ & $20,93 \%$ & $18,38 \%$ \\
\hline "Chance" I & 2,653 & 2,653 & 0,898 & 0,898 & 0 & $74,66 \%$ & $15,04 \%$ & $10,29 \%$ \\
\hline "Chance" II & 0,436 & 2,36 & $-0,738$ & 1,187 & $-1,924$ & & & \\
\hline Implícito I & 1,8 & 1,8 & 1,1 & 1,1 & 0 & $53,76 \%$ & $23,14 \%$ & $23,10 \%$ \\
\hline Implícito II & 1,8 & 1,426 & 1,1 & 0,726 & 0,374 & $53,80 \%$ & $27,16 \%$ & $19,04 \%$ \\
\hline Implícito III & 2 & 2 & 0,2 & 0,2 & 0 & $81,06 \%$ & $15,98 \%$ & $2,96 \%$ \\
\hline
\end{tabular}

França 3x0 Brasil

\begin{tabular}{|c|c|c|c|c|c|c|c|c|}
\hline SD 0 & 1,284 & 1,284 & 1,096 & 1,096 & 0 & $40,75 \%$ & $27,64 \%$ & $31,61 \%$ \\
\hline SD I & 1,284 & 0,898 & 1,096 & 0,709 & 0,387 & $37,86 \%$ & $35,00 \%$ & $27,14 \%$ \\
\hline "Chance" I & 1,052 & 1,052 & 0,845 & 0,845 & 0 & $39,74 \%$ & $31,61 \%$ & $28,65 \%$ \\
\hline "Chance" II & $-0,335$ & 1,589 & $-0,575$ & 1,35 & $-1,924$ & & & \\
\hline Implícito I & 1,4 & 1,4 & 2 & 2 & 0 & $26,86 \%$ & $21,66 \%$ & $51,48 \%$ \\
\hline Implícito II & 1,4 & 0,207 & 2 & 0,807 & 1,193 & $9,04 \%$ & $42,58 \%$ & $48,37 \%$ \\
\hline Implícito III & 2 & 2 & 1 & 1 & 0 & $60,57 \%$ & $21,17 \%$ & $18,26 \%$ \\
\hline
\end{tabular}


A.2. Jogos do Campeonato Brasileiro de 1998
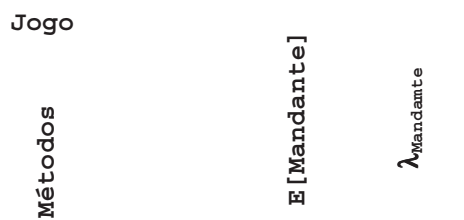
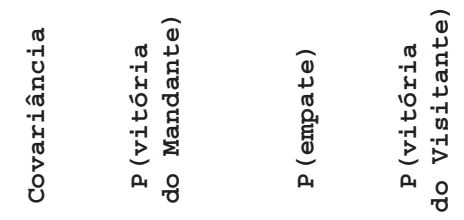

Vasco 0x1 Corinthians

$\begin{array}{lrrrrrrrr}\text { SD 0 } & 1,818 & 1,818 & 0,518 & 0,518 & 0 & 68,99 \% & 21,16 \% & 9,85 \% \\ \text { SD I } & 1,818 & 1,998 & 0,518 & 0,698 & -0,18 & & & \\ \text { "Chance" I } & 1,839 & 1,839 & 0,69 & 0,69 & 0 & 64,84 \% & 21,80 \% & 13,36 \% \\ \text { "Chance" II } & 3,733 & 1,806 & 2,476 & 0,55 & 1,926 & 67,72 \% & 21,50 \% & 10,61 \% \\ \text { Implícito I } & 1,9 & 1,9 & 1,1 & 1,1 & 0 & 56,03 \% & 22,26 \% & 21,71 \% \\ \text { Implícito II } & 1,9 & 0,94 & 1,1 & 0,14 & 0,96 & 55,98 \% & 38,57 \% & 5,45 \%\end{array}$

Flamengo 1x1 Botafogo

$\begin{array}{lrrrrrrrr}\text { SD 0 } & 1,428 & 1,428 & 1,993 & 1,993 & 0 & 27,57 \% & 21,70 \% & 50,73 \% \\ \text { SD I } & 1,428 & 1,58 & 1,993 & 2,145 & -0,151 & & & \\ \text { "Chance" I } & 1,538 & 1,538 & 2,233 & 2,233 & 0 & 26,33 \% & 20,23 \% & 53,44 \% \\ \text { "Chance" II } & 3,482 & 1,556 & 4,04 & 2,114 & 1,926 & 28,37 \% & 20,92 \% & 50,32 \% \\ \text { Implícito I } & 1,4 & 1,4 & 1,3 & 1,3 & 0 & 39,41 \% & 25,78 \% & 34,81 \% \\ \text { Implícito II } & 1,4 & 0,74 & 1,3 & 0,64 & 0,66 & 33,75 \% & 38,55 \% & 27,70 \%\end{array}$

Atlético MG $1 \times 1$ Cruzeiro

\begin{tabular}{|c|c|c|c|c|c|c|c|c|}
\hline $\mathrm{SD} 0$ & 1,295 & 1,295 & 1,279 & 1,279 & 0 & $37,10 \%$ & $26,55 \%$ & $36,35 \%$ \\
\hline SD I & 1,295 & 1,286 & 1,279 & 1,27 & 0,009 & $37,05 \%$ & $26,66 \%$ & $36,29 \%$ \\
\hline "Chance" I & 1,363 & 1,363 & 1,356 & 1,356 & 0 & $37,30 \%$ & $25,71 \%$ & $36,99 \%$ \\
\hline "Chance" II & 3,335 & 1,409 & 3,315 & 1,389 & 1,926 & $37,73 \%$ & $25,29 \%$ & $36,85 \%$ \\
\hline Implícito I & 1,6 & 1,6 & 1,6 & 1,6 & 0 & $38,29 \%$ & $23,43 \%$ & $38,29 \%$ \\
\hline Implícito II & 1,6 & 0,52 & 1,6 & 0,52 & 1,08 & $27,22 \%$ & $45,56 \%$ & $27,22 \%$ \\
\hline Internacional $1 \times 0$ & Grêmio & & & & & & & \\
\hline $\mathrm{SD} 0$ & 2,417 & 2,417 & 1,088 & 1,088 & 0 & $66,67 \%$ & $17,90 \%$ & $15,43 \%$ \\
\hline SD I & 2,417 & 2,577 & 1,088 & 1,247 & $-0,159$ & & & \\
\hline "Chance" I & 2,884 & 2,884 & 1,173 & 1,173 & 0 & $72,49 \%$ & $14,85 \%$ & $12,64 \%$ \\
\hline "Chance" II & 4,502 & 2,576 & 3,167 & 1,241 & 1,926 & $65,52 \%$ & $17,29 \%$ & $16,49 \%$ \\
\hline Implícito I & 2 & 2 & 1,2 & 1,2 & 0 & $55,91 \%$ & $21,55 \%$ & $22,54 \%$ \\
\hline Implícito II & 2 & 0,766 & 1,2 & $-0,034$ & 1,234 & & & \\
\hline
\end{tabular}

Atlético PR $2 \times 2$ Coritiba

$\begin{array}{lrrrrrrrr}\text { SD 0 } & 1,508 & 1,508 & 1,213 & 1,213 & 0 & 44,06 \% & 25,38 \% & 30,56 \% \\ \text { SD I } & 1,508 & 1,791 & 1,213 & 1,496 & -0,283 & & & \\ \text { "Chance" I } & 1,567 & 1,567 & 1,236 & 1,236 & 0 & 45,04 \% & 24,87 \% & 30,09 \% \\ \text { "Chance" II } & 3,482 & 1,556 & 3,193 & 1,267 & 1,926 & 43,98 \% & 24,86 \% & 31,01 \% \\ \text { Implícito I } & 1,9 & 1,9 & 1,6 & 1,6 & 0 & 45,11 \% & 22,04 \% & 32,85 \% \\ \text { Implícito II } & 1,9 & 0,52 & 1,6 & 0,22 & 1,38 & 34,28 \% & 53,31 \% & 12,41 \%\end{array}$


Juventude 3x0 Paraná

$\begin{array}{lrrrrrrrr}\text { SD 0 } & 1,349 & 1,349 & 0,272 & 0,272 & 0 & 64,95 \% & 27,72 \% & 7,33 \% \\ \text { SD I } & 1,349 & 1,287 & 0,272 & 0,21 & 0,062 & 65,21 \% & 28,85 \% & 5,94 \% \\ \text { "Chance" I } & 1,248 & 1,248 & 0,541 & 0,541 & 0 & 53,86 \% & 30,05 \% & 16,09 \% \\ \text { "Chance" II } & 3,225 & 1,298 & 2,192 & 0,265 & 1,926 & 63,69 \% & 28,79 \% & 7,47 \% \\ \text { Implícito I } & 1,8 & 1,8 & 1,1 & 1,1 & 0 & 53,76 \% & 23,14 \% & 23,10 \% \\ \text { Implícito II } & 1,8 & 1,107 & 1,1 & 0,407 & 0,693 & 53,36 \% & 33,08 \% & 13,55 \%\end{array}$

Guarani 2x0 Ponte Preta

$\begin{array}{lrrrrrrrr}\text { SD 0 } & 0,667 & 0,667 & 0,21 & 0,21 & 0 & 41,97 \% & 47,64 \% & 10,39 \% \\ \text { SD I } & 0,667 & 1,321 & 0,21 & 0,864 & -0,654 & & & \\ \text { "Chance" I } & 0,581 & 0,581 & 0,581 & 0,581 & 0 & 28,62 \% & 42,76 \% & 28,61 \% \\ \text { "Chance" II } & 2,721 & 0,795 & 2,281 & 0,354 & 1,926 & 43,38 \% & 41,26 \% & 15,34 \% \\ \text { Implícito I } & 1,6 & 1,6 & 1,5 & 1,5 & 0 & 40,26 \% & 23,82 \% & 35,92 \% \\ \text { Implícito II } & 1,6 & 0,803 & 1,5 & 0,703 & 0,797 & 34,63 \% & 36,59 \% & 28,78 \%\end{array}$

Implícito II $\quad \begin{array}{lllllll}1,6 & 0,803 & 1,5 & 0,703 & 0,797 & 34,63 \% & 36,59 \%\end{array}$

Santos 2x0 Bragantino

$\begin{array}{lrrrrrrrr}\text { SD 0 } & 2,504 & 2,504 & 0,607 & 0,607 & 0 & 78,76 \% & 14,28 \% & 6,95 \% \\ \text { SD I } & 2,504 & 3,156 & 0,607 & 1,259 & -0,652 & & & \\ \text { "Chance" I } & 2,835 & 2,835 & 0,737 & 0,737 & 0 & 80,47 \% & 12,56 \% & 6,95 \% \\ \text { "Chance" II } & 4,45 & 2,523 & 2,584 & 0,657 & 1,926 & 77,32 \% & 14,49 \% & 7,57 \% \\ \text { Implícito I } & 1,9 & 1,9 & 1 & 1 & 0 & 58,41 \% & 22,11 \% & 19,49 \% \\ \text { Implícito II } & 1,9 & 1,303 & 1 & 0,403 & 0,597 & 59,56 \% & 29,03 \% & 11,41 \%\end{array}$

Portuguesa 2x1 América MG

$\begin{array}{lrrrrrrrr}\text { SD 0 } & 2,11 & 2,11 & 0,779 & 0,779 & 0 & 68,14 \% & 19,23 \% & 12,63 \% \\ \text { SD I } & 2,11 & 1,099 & 0,779 & -0,232 & 1,011 & & & \\ \text { "Chance" I } & 2,088 & 2,088 & 0,812 & 0,812 & 0 & 66,91 \% & 19,62 \% & 13,47 \% \\ \text { "Chance" II } & 3,95 & 2,024 & 2,661 & 0,735 & 1,926 & 67,30 \% & 19,90 \% & 12,54 \% \\ \text { Implícito I } & 1,7 & 1,7 & 1,4 & 1,4 & 0 & 44,69 \% & 23,57 \% & 31,74 \% \\ \text { Implícito II } & 1,7 & 0,807 & 1,4 & 0,507 & 0,893 & 39,66 \% & 39,04 \% & 21,30 \%\end{array}$

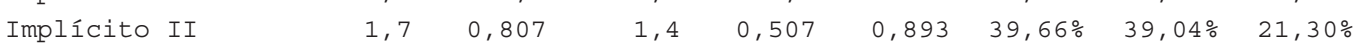

América RN 0x4 Sport

$\begin{array}{lrrrrrrrr}\text { SD 0 } & 1,648 & 1,648 & 1,295 & 1,295 & 0 & 45,72 \% & 24,15 \% & 30,13 \% \\ \text { SD I } & 1,648 & 2,083 & 1,295 & 1,729 & -0,434 & & & \\ \text { "Chance" I } & 1,593 & 1,593 & 1,27 & 1,27 & 0 & 44,93 \% & 24,60 \% & 30,48 \% \\ \text { "Chance" II } & 3,565 & 1,638 & 3,264 & 1,338 & 1,926 & 44,43 \% & 24,11 \% & 31,29 \% \\ \text { Implícito I } & 1,8 & 1,8 & 1,7 & 1,7 & 0 & 40,92 \% & 22,25 \% & 36,82 \% \\ \text { Implícito }\end{array}$

Implícito II $\quad 1,8 \quad 0,42 \quad 1,7 \quad 0,32 \quad 1,38 \quad 26,50 \% \quad 54,32 \%$

Vitória 1x2 Goiás

$\begin{array}{lrrrrrrrr}\text { SD 0 } & 2,316 & 2,316 & 1,167 & 1,167 & 0 & 63,06 \% & 18,98 \% & 17,96 \% \\ \text { SD I } & 2,316 & 2,52 & 1,167 & 1,371 & -0,204 & & & \\ \text { "Chance" I } & 2,524 & 2,524 & 1,094 & 1,094 & 0 & 68,40 \% & 17,08 \% & 14,51 \% \\ \text { "Chance" II } & 4,241 & 2,315 & 3,131 & 1,204 & 1,926 & 61,78 \% & 19,11 \% & 18,65 \% \\ \text { Implícito I } & 1,8 & 1,8 & 1,6 & 1,6 & 0 & 42,88 \% & 22,53 \% & 34,58 \% \\ \text { Implícito II } & 1,8 & 0,808 & 1,6 & 0,608 & 0,992 & 37,11 \% & 37,74 \% & 25,15 \%\end{array}$


Palmeiras 2x1 São Paulo

$\begin{array}{lrrrrrrrr}\text { SD 0 } & 1,585 & 1,585 & 1,38 & 1,38 & 0 & 42,36 \% & 24,32 \% & 33,31 \% \\ \text { SD I } & 1,585 & 1,825 & 1,38 & 1,62 & -0,24 & & & \\ \text { "Chance" I } & 1,728 & 1,728 & 1,438 & 1,438 & 0 & 44,57 \% & 23,31 \% & 32,12 \% \\ \text { "Chance" II } & 3,663 & 1,736 & 3,446 & 1,52 & 1,926 & 42,92 \% & 23,06 \% & 33,80 \% \\ \text { Implícito I } & 1,4 & 1,4 & 1,6 & 1,6 & 0 & 33,52 \% & 24,17 \% & 42,31 \% \\ \text { Implícito II } & 1,4 & 0,808 & 1,6 & 1,008 & 0,592 & 28,33 \% & 32,47 \% & 39,20 \%\end{array}$

Santos 1x1 Atlético PR

$\begin{array}{lrrrrrrrr}\text { SD 0 } & 1,861 & 1,861 & 0,958 & 0,958 & 0 & 58,56 \% & 22,40 \% & 19,05 \% \\ \text { SD I } & 1,861 & 2,458 & 0,958 & 1,555 & -0,597 & & & \\ \text { "Chance" I } & 1,965 & 1,965 & 1,003 & 1,003 & 0 & 59,73 \% & 21,51 \% & 18,76 \% \\ \text { "Chance" II } & 3,785 & 1,858 & 2,922 & 0,994 & 1,928 & 57,41 \% & 22,49 \% & 19,89 \% \\ \text { Implícito I } & 2 & 2 & 1,4 & 1,4 & 0 & 51,48 \% & 21,66 \% & 26,86 \% \\ \text { Implícito II } & 2 & 0,903 & 1,4 & 0,303 & 1,097 & 49,25 \% & 38,72 \% & 12,02 \%\end{array}$

Atlético MG 1x1 Guarani

$\begin{array}{lrrrrrrrr}\text { SD 0 } & 1,607 & 1,607 & 1,054 & 1,054 & 0 & 50,22 \% & 24,89 \% & 24,90 \% \\ \text { SD I } & 1,607 & 1,167 & 1,054 & 0,614 & 0,44 & 49,31 \% & 31,26 \% & 19,43 \% \\ \text { "Chance" I } & 1,526 & 1,526 & 1,05 & 1,05 & 0 & 48,27 \% & 25,64 \% & 26,09 \% \\ \text { "Chance" II } & 3,497 & 1,569 & 2,982 & 1,054 & 1,928 & 49,18 \% & 25,23 \% & 25,46 \% \\ \text { Implícito I } & 1,8 & 1,8 & 1,4 & 1,4 & 0 & 47,02 \% & 22,95 \% & 30,03 \% \\ \text { Implícito II } & 1,8 & 1,126 & 1,4 & 0,726 & 0,674 & 45,02 \% & 31,40 \% & 23,58 \%\end{array}$

Coritiba 1x3 Flamengo

\begin{tabular}{|c|c|c|c|c|c|c|c|c|}
\hline SD 0 & 1,727 & 1,727 & 1,113 & 1,113 & 0 & $51,74 \%$ & $23,77 \%$ & $24,49 \%$ \\
\hline $\mathrm{SD} I$ & 1,727 & 1,243 & 1,113 & 0,629 & 0,484 & $51,22 \%$ & $29,97 \%$ & $18,81 \%$ \\
\hline "Chance" I & 1,497 & 1,497 & 1,048 & 1,048 & 0 & $47,56 \%$ & $25,92 \%$ & $26,52 \%$ \\
\hline "Chance" II & 0,773 & 0,221 & 0,229 & $-0,324$ & 0,552 & & & \\
\hline Implícito I & 1,7 & 1,7 & 1,7 & 1,7 & 0 & $38,68 \%$ & $22,64 \%$ & $38,68 \%$ \\
\hline Implícito II & 1,7 & 0,603 & 1,7 & 0,603 & 1,097 & $29,06 \%$ & $41,87 \%$ & $29,06 \%$ \\
\hline \multicolumn{9}{|c|}{ Goiás lxl Palmeiras } \\
\hline SD 0 & 1,06 & 1,06 & 1,498 & 1,498 & 0 & $26,81 \%$ & $25,88 \%$ & $47,31 \%$ \\
\hline $\mathrm{SD} I$ & 1,06 & 1,298 & 1,498 & 1,737 & $-0,238$ & & & \\
\hline "Chance" I & 0,983 & 0,983 & 1,468 & 1,468 & 0 & $25,30 \%$ & $26,33 \%$ & $48,37 \%$ \\
\hline "Chance" II & 0,195 & $-0,357$ & 0,727 & 0,174 & 0,552 & & & \\
\hline Implícito I & 1 & 1 & 1,9 & 1,9 & 0 & $19,49 \%$ & $22,11 \%$ & $58,41 \%$ \\
\hline Implícito II & 1 & 0,307 & 1,9 & 1,207 & 0,693 & $9,39 \%$ & $30,94 \%$ & $59,67 \%$ \\
\hline \multicolumn{9}{|c|}{ Bragantino 0x0 Vasco } \\
\hline SD 0 & 1,03 & 1,03 & 1,873 & 1,873 & 0 & $20,50 \%$ & $22,41 \%$ & $57,08 \%$ \\
\hline $\mathrm{SD} I$ & 1,03 & 0,875 & 1,873 & 1,718 & 0,155 & $18,95 \%$ & $23,72 \%$ & $57,32 \%$ \\
\hline "Chance" I & 0,921 & 0,921 & 1,821 & 1,821 & 0 & $18,72 \%$ & $22,73 \%$ & $58,55 \%$ \\
\hline "Chance" II & 0,181 & $-0,371$ & 1,117 & 0,564 & 0,552 & & & \\
\hline Implícito I & 0,9 & 0,9 & 1,8 & 1,8 & 0 & $18,50 \%$ & $22,90 \%$ & $58,61 \%$ \\
\hline Implícito II & 0,9 & 0,726 & 1,8 & 1,626 & 0,174 & $16,49 \%$ & $24,54 \%$ & $58,97 \%$ \\
\hline
\end{tabular}


Corinthians $4 \times 0$ Juventude

$\begin{array}{lrrrrrrrr}\text { SD 0 } & 1,039 & 1,039 & 0,696 & 0,696 & 0 & 43,00 \% & 32,90 \% & 24,10 \% \\ \text { SD I } & 1,039 & 1,588 & 0,696 & 1,246 & -0,55 & & & \\ \text { "Chance" I } & 0,936 & 0,936 & 0,812 & 0,812 & 0 & 36,72 \% & 33,39 \% & 29,89 \% \\ \text { "Chance" II } & 0,177 & -0,375 & -0,087 & -0,64 & 0,552 & & & \\ \text { Implícito I } & 1,6 & 1,6 & 1,6 & 1,6 & 0 & 38,29 \% & 23,43 \% & 38,29 \% \\ \text { Implícito II } & 1,6 & 0,607 & 1,6 & 0,607 & 0,993 & 29,16 \% & 41,68 \% & 29,16 \%\end{array}$

São Paulo $2 \times 1$ Guarani

$\begin{array}{lrrrrrrrr}\text { SD 0 } & 1,768 & 1,768 & 0,826 & 0,826 & 0 & 59,74 \% & 23,08 \% & 17,19 \% \\ \text { SD I } & 1,768 & 1,017 & 0,826 & 0,075 & 0,751 & 61,12 \% & 36,16 \% & 2,71 \% \\ \text { "Chance" I } & 1,682 & 1,682 & 0,887 & 0,887 & 0 & 56,14 \% & 24,14 \% & 19,72 \% \\ \text { "Chance" II } & 0,883 & 0,331 & 0,016 & -0,537 & 0,552 & & & \\ \text { Implícito I } & 2 & 2 & 1,6 & 1,6 & 0 & 47,29 \% & 21,53 \% & 31,18 \% \\ \text { Implícito II } & 2 & 1,059 & 1,6 & 0,659 & 0,941 & 44,63 \% & 32,82 \% & 22,55 \%\end{array}$

Paraná 0x1 Portuguesa

$\begin{array}{lrrrrrrrr}\text { SD 0 } & 1,255 & 1,255 & 1,214 & 1,214 & 0 & 37,38 \% & 27,20 \% & 35,42 \% \\ \text { SD I } & 1,255 & 0,692 & 1,214 & 0,65 & 0,564 & 31,61 \% & 39,28 \% & 29,11 \% \\ \text { "Chance" I } & 1,092 & 1,092 & 1,161 & 1,161 & 0 & 33,95 \% & 28,70 \% & 37,35 \% \\ \text { "Chance" II } & 0,323 & -0,229 & 0,355 & -0,198 & 0,552 & & & \\ \text { Implícito I } & 1,2 & 1,2 & 1,8 & 1,8 & 0 & 25,42 \% & 23,14 \% & 51,44 \% \\ \text { Implícito II } & 1,2 & 0,503 & 1,8 & 1,103 & 0,697 & 16,75 \% & 32,86 \% & 50,39 \%\end{array}$

Botafogo 1x2 Vitória

\begin{tabular}{|c|c|c|c|c|c|c|c|c|}
\hline SD 0 & 2,223 & 2,223 & 1,226 & 1,226 & 0 & $59,94 \%$ & $19,87 \%$ & $20,19 \%$ \\
\hline SD I & 2,223 & 2,144 & 1,226 & 1,147 & 0,079 & $60,13 \%$ & $20,30 \%$ & $19,56 \%$ \\
\hline "Chance" I & 2,409 & 2,409 & 1,174 & 1,174 & 0 & $64,63 \%$ & $18,29 \%$ & $17,08 \%$ \\
\hline "Chance" II & 1,36 & 0,808 & 0,441 & $-0,111$ & 0,552 & & & \\
\hline Implícito I & 2 & 2 & 1,2 & 1,2 & 0 & $55,91 \%$ & $21,55 \%$ & $22,54 \%$ \\
\hline Implícito II & 2 & 1,208 & 1,2 & 0.408 & 0.792 & $56,56 \%$ & $30,94 \%$ & $12,50 \%$ \\
\hline
\end{tabular}

$\begin{array}{lllllll}\text { Implícito II } \quad 2 & 1,208 & 1,2 & 0,408 & 0,792 & 56,56 \% & 30,94 \%\end{array}$

América MG 2x2 América RN

$\begin{array}{lrrrrrrrr}\text { SD 0 } & 1,836 & 1,836 & 1,07 & 1,07 & 0 & 55,30 \% & 22,80 \% & 21,91 \% \\ \text { SD I } & 1,836 & 1,337 & 1,07 & 0,571 & 0,499 & 55,60 \% & 28,52 \% & 15,88 \% \\ \text { "Chance" I } & 1,733 & 1,733 & 0,977 & 0,977 & 0 & 55,14 \% & 23,69 \% & 21,17 \% \\ \text { "Chance" II } & 0,968 & 0,415 & 0,245 & -0,307 & 0,552 & & & \\ \text { Implícito I } & 2 & 2 & 0,9 & 0,9 & 0 & 62,98 \% & 20,86 \% & 16,16 \% \\ \text { Implícito II } & 2 & 1,508 & 0,9 & 0,408 & 0,492 & 64,97 \% & 25,27 \% & 9,76 \%\end{array}$

Ponte Preta 0x0 Internacional

$\begin{array}{lrrrrrrrr}\text { SD 0 } & 0,147 & 0,147 & 0,795 & 0,795 & 0 & 6,53 \% & 43,68 \% & 49,79 \% \\ \text { SD I } & 0,147 & 0,855 & 0,795 & 1,503 & -0,708 & & & \\ \text { "Chance" I } & 0,379 & 0,379 & 0,773 & 0,773 & 0 & 16,64 \% & 41,57 \% & 41,79 \% \\ \text { "Chance" II } & -0,797 & -1,35 & -0,048 & -0,6 & 0,552 & & & \\ \text { Implícito I } & 1,3 & 1,3 & 1,8 & 1,8 & 0 & 27,73 \% & 23,07 \% & 49,19 \% \\ \text { Implícito II } & 1,3 & 0,503 & 1,8 & 1,003 & 0,797 & 18,12 \% & 34,87 \% & 47,00 \%\end{array}$


Sport 2xO Atlético PR

$\begin{array}{lrrrrrrrr}\text { SD 0 } & 1,964 & 1,964 & 1,2 & 1,2 & 0 & 55,13 \% & 21,84 \% & 23,03 \% \\ \text { SD I } & 1,964 & 2,718 & 1,2 & 1,954 & -0,754 & & & \\ \text { "Chance" I } & 2,034 & 2,034 & 1,175 & 1,175 & 0 & 57,22 \% & 21,25 \% & 21,54 \% \\ \text { "Chance" II } & 1,076 & 0,524 & 0,37 & -0,182 & 0,552 & & & \\ \text { Implícito I } & 1,8 & 1,8 & 1,5 & 1,5 & 0 & 44,92 \% & 22,77 \% & 32,32 \% \\ \text { Implícito II } & 1,8 & 0,707 & 1,5 & 0,407 & 1,093 & 38,23 \% & 42,96 \% & 18,81 \%\end{array}$

Grêmio 2x3 Atlético MG

$\begin{array}{lrrrrrrrr}\text { SD 0 } & 1,502 & 1,502 & 1,712 & 1,712 & 0 & 33,91 \% & 23,24 \% & 42,85 \% \\ \text { SD I } & 1,502 & 1,345 & 1,712 & 1,555 & 0,157 & 33,01 \% & 24,62 \% & 42,37 \% \\ \text { "Chance" I } & 1,433 & 1,433 & 1,563 & 1,563 & 0 & 35,01 \% & 24,26 \% & 40,73 \% \\ \text { "Chance" II } & 0,625 & 0,072 & 0,894 & 0,341 & 0,552 & 5,02 \% & 67,76 \% & 27,22 \% \\ \text { Implícito I } & 1,6 & 1,6 & 1,5 & 1,5 & 0 & 40,26 \% & 23,82 \% & 35,92 \% \\ \text { Implícito II } & 1,6 & 0,803 & 1,5 & 0,703 & 0,797 & 34,63 \% & 36,59 \% & 28,78 \%\end{array}$

Cruzeiro 1x2 Santos

\begin{tabular}{|c|c|c|c|c|c|c|c|c|}
\hline SD 0 & 1,278 & 1,278 & 0,85 & 0,85 & 0 & $46,42 \%$ & $28,81 \%$ & $24,77 \%$ \\
\hline SD I & 1,278 & 1,315 & 0,85 & 0,887 & $-0,037$ & & & \\
\hline "Chance" I & 1,209 & 1,209 & 0,908 & 0,908 & 0 & $43,00 \%$ & $29,36 \%$ & $27,64 \%$ \\
\hline "Chance" II & 0,422 & $-0,13$ & 0,06 & $-0,493$ & 0,552 & & & \\
\hline Implícito I & 1,8 & 1,8 & 1,4 & 1,4 & 0 & $47,02 \%$ & $22,95 \%$ & $30,03 \%$ \\
\hline Implícito II & 1,8 & 1,008 & 1,4 & 0,608 & 0,792 & $44,28 \%$ & $34,05 \%$ & $21,67 \%$ \\
\hline Guarani $2 \times 3$ & Botafogo & & & & & & & \\
\hline SD 0 & 1,498 & 1,498 & 0,914 & 0,914 & 0 & $50,86 \%$ & $26,13 \%$ & $23,01 \%$ \\
\hline SD I & 1,498 & 0,949 & 0,914 & 0,364 & 0,55 & $49,10 \%$ & $37,04 \%$ & $13,86 \%$ \\
\hline "Chance" I & 1,432 & 1,432 & 0,949 & 0,949 & 0 & $48,24 \%$ & $26,78 \%$ & $24,98 \%$ \\
\hline "Chance" II & 3,352 & 1,453 & 2,843 & 0,944 & 1,899 & $48,85 \%$ & $26,57 \%$ & $24,49 \%$ \\
\hline Implícito I & 1,3 & 1,3 & 1 & 1 & 0 & $43,40 \%$ & $27,96 \%$ & $28,64 \%$ \\
\hline Implícito II & 1,3 & 1,303 & 1 & 1,003 & $-0,003$ & & & \\
\hline
\end{tabular}

Internacional 0x3 são Paulo

$\begin{array}{lrrrrrrrr}\text { SD 0 } & 1,607 & 1,607 & 0,955 & 0,955 & 0 & 52,62 \% & 24,95 \% & 22,43 \% \\ \text { SD I } & 1,607 & 0,93 & 0,955 & 0,278 & 0,677 & 51,07 \% & 38,14 \% & 10,79 \% \\ \text { "Chance" I } & 2,325 & 2,325 & 0,969 & 0,969 & 0 & 67,67 \% & 18,14 \% & 14,19 \% \\ \text { "Chance" II } & 4,06 & 2,162 & 2,999 & 1,1 & 1,899 & 61,25 \% & 20,03 \% & 18,39 \% \\ \text { Implícito I } & 1,3 & 1,3 & 1,3 & 1,3 & 0 & 36,80 \% & 26,39 \% & 36,80 \% \\ \end{array}$

Vasco 5x1 América MG

$\begin{array}{lrrrrrrrr}\text { SD 0 } & 2,176 & 2,176 & 0,635 & 0,635 & 0 & 72,88 \% & 17,68 \% & 9,44 \% \\ \text { SD I } & 2,176 & 1,477 & 0,635 & -0,064 & 0,699 & & & \\ \text { "Chance" I } & 2,244 & 2,244 & 0,75 & 0,75 & 0 & 71,32 \% & 17,72 \% & 10,96 \% \\ \text { "Chance" II } & 4,014 & 2,115 & 2,542 & 0,643 & 1,899 & 71,29 \% & 18,38 \% & 10,03 \% \\ \text { Implícito I } & 2 & 2 & 1,2 & 1,2 & 0 & 55,91 \% & 21,55 \% & 22,54 \% \\ \text { Implícito II } & 2 & 1,208 & 1,2 & 0,408 & 0,792 & 56,56 \% & 30,94 \% & 12,50 \%\end{array}$


Atlético PR 0x1 Palmeiras

$\begin{array}{lrrrrrrrr}\text { SD 0 } & 1,272 & 1,272 & 1,476 & 1,476 & 0 & 32,64 \% & 25,40 \% & 41,95 \% \\ \text { SD I } & 1,272 & 1,643 & 1,476 & 1,847 & -0,371 & & & \\ \text { "Chance" I } & 1,136 & 1,136 & 1,406 & 1,406 & 0 & 30,41 \% & 26,45 \% & 43,14 \% \\ \text { "Chance" II } & 3,077 & 1,178 & 3,346 & 1,447 & 1,899 & 30,73 \% & 25,96 \% & 43,21 \% \\ \text { Implícito I } & 1 & 1 & 1,4 & 1,4 & 0 & 26,88 \% & 26,97 \% & 46,15 \% \\ \text { Implícito II } & 1 & 0,903 & 1,4 & 1,303 & 0,097 & 25,85 \% & 28,33 \% & 45,82 \%\end{array}$

Corinthians $2 \times 1$ Grêmio

$\begin{array}{lrrrrrrrr}\text { SD 0 } & 2,225 & 2,225 & 0,738 & 0,738 & 0 & 71,26 \% & 17,84 \% & 10,90 \% \\ \text { SD I } & 2,225 & 2,203 & 0,738 & 0,716 & 0,022 & 71,40 \% & 17,92 \% & 10,68 \% \\ \text { "Chance" I } & 2,191 & 2,191 & 0,833 & 0,833 & 0 & 68,38 \% & 18,70 \% & 12,91 \% \\ \text { "Chance" II } & 4,07 & 2,171 & 2,652 & 0,753 & 1,899 & 69,62 \% & 18,46 \% & 11,60 \% \\ \text { Implícito I } & 2,2 & 2,2 & 0,9 & 0,9 & 0 & 66,96 \% & 18,94 \% & 14,10 \% \\ \text { Implícito II } & 2,2 & 1,626 & 0,9 & 0,326 & 0,574 & 70,25 \% & 22,79 \% & 6,96 \%\end{array}$

Portuguesa $1 \times 1$ Coritiba

$\begin{array}{lrrrrrrrr}\text { SD 0 } & 1,897 & 1,897 & 0,587 & 0,587 & 0 & 68,79 \% & 20,56 \% & 10,65 \% \\ \text { SD I } & 1,897 & 1,317 & 0,587 & 0,006 & 0,581 & 72,96 \% & 26,86 \% & 0,17 \% \\ \text { "Chance" I } & 1,822 & 1,822 & 0,698 & 0,698 & 0 & 64,27 \% & 22,03 \% & 13,70 \% \\ \text { "Chance" II } & 3,662 & 1,764 & 2,44 & 0,541 & 1,899 & 67,04 \% & 22,03 \% & 10,78 \% \\ \text { Implícito I } & 1,7 & 1,7 & 1 & 1 & 0 & 53,79 \% & 24,02 \% & 22,18 \% \\ \text { Implícito II } & 1,7 & 1,207 & 1 & 0,507 & 0,493 & 53,59 \% & 30,84 \% & 15,58 \%\end{array}$

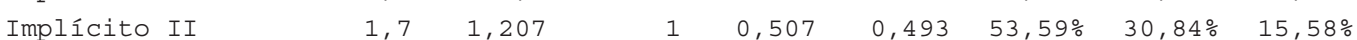

Cruzeiro 4x2 Ponte Preta

\begin{tabular}{|c|c|c|c|c|c|c|c|c|}
\hline SD 0 & 0,869 & 0,869 & $-0,37$ & $-0,37$ & 0 & & & \\
\hline $\mathrm{SD} I$ & 0,869 & 1,867 & $-0,37$ & 0,628 & $-0,998$ & & & \\
\hline "Chance" I & 0,707 & 0,707 & 0,243 & 0,243 & 0 & $42,87 \%$ & $45,60 \%$ & $11,54 \%$ \\
\hline "Chance" II & 2,644 & 0,745 & 1,479 & $-0,42$ & 1,899 & & & \\
\hline Implícito I & 2,1 & 2,1 & 0,8 & 0,8 & 0 & $67,44 \%$ & $19,43 \%$ & $13,13 \%$ \\
\hline Implícito II & 2,1 & 1,608 & 0,8 & 0,308 & 0,492 & $70,37 \%$ & $22,96 \%$ & $6,66 \%$ \\
\hline
\end{tabular}

Paraná 3x2 América RN

$\begin{array}{lrrrrrrrr}\text { SD 0 } & 1,565 & 1,565 & 0,853 & 0,853 & 0 & 54,13 \% & 25,41 \% & 20,46 \% \\ \text { SD I } & 1,565 & 1,349 & 0,853 & 0,637 & 0,216 & 54,09 \% & 28,30 \% & 17,61 \% \\ \text { "Chance" I } & 1,385 & 1,385 & 0,894 & 0,894 & 0 & 48,33 \% & 27,43 \% & 24,24 \% \\ \text { "Chance" II } & 3,428 & 1,529 & 2,74 & 0,841 & 1,899 & 53,44 \% & 25,83 \% & 20,64 \% \\ \text { Implícito I } & 0,9 & 0,9 & 0,8 & 0,8 & 0 & 35,80 \% & 33,99 \% & 30,21 \% \\ \text { Implícito II } & 0,9 & 1,24 & 0,8 & 1,14 & -0,34 & & & \end{array}$

Sport 2x1 Vitória

$\begin{array}{lrrrrrrrr}\text { SD 0 } & 2,282 & 2,282 & 1,468 & 1,468 & 0 & 55,88 \% & 19,87 \% & 24,24 \% \\ \text { SD I } & 2,282 & 3,291 & 1,468 & 2,478 & -1,01 & & & \\ \text { "Chance" I } & 2,599 & 2,599 & 1,413 & 1,413 & 0 & 62,99 \% & 17,71 \% & 19,29 \% \\ \text { "Chance" II } & 4,196 & 2,297 & 3,417 & 1,518 & 1,899 & 54,74 \% & 19,83 \% & 24,96 \% \\ \text { Implícito I } & 1,4 & 1,4 & 1,3 & 1,3 & 0 & 39,41 \% & 25,78 \% & 34,81 \% \\ \text { Implícito II } & 1,4 & 1,108 & 1,3 & 1,008 & 0,292 & 37,67 \% & 29,77 \% & 32,56 \%\end{array}$


Atlético MG $1 \times 5$ Corinthians

$\begin{array}{lrrrrrrrr}\text { SD 0 } & 1,566 & 1,566 & 0,974 & 0,974 & 0 & 51,12 \% & 25,36 \% & 23,52 \% \\ \text { SD I } & 1,566 & 2,019 & 0,974 & 1,427 & -0,453 & & & \\ \text { "Chance" I } & 1,429 & 1,429 & 1 & 1 & 0 & 46,93 \% & 26,69 \% & 26,38 \% \\ \text { "Chance" II } & 3,3 & 1,466 & 2,816 & 0,981 & 1,834 & 48,30 \% & 26,36 \% & 25,27 \% \\ \text { Implícito I } & 1,3 & 1,3 & 1,8 & 1,8 & 0 & 27,73 \% & 23,07 \% & 49,19 \% \\ \text { Implícito II } & 1,3 & 0,859 & 1,8 & 1,359 & 0,441 & 23,70 \% & 27,81 \% & 48,49 \%\end{array}$

Coritiba $2 \times 2$ Ponte Preta

$\begin{array}{lrrrrrrrr}\text { SD 0 } & 1,328 & 1,328 & 0,306 & 0,306 & 0 & 63,29 \% & 28,29 \% & 8,42 \% \\ \text { SD I } & 1,328 & 0,573 & 0,306 & -0,449 & 0,755 & & & \\ \text { "Chance" I } & 1,2 & 1,2 & 0,493 & 0,493 & 0 & 53,77 \% & 31,01 \% & 15,22 \% \\ \text { "Chance" II } & 3,029 & 1,195 & 2,058 & 0,223 & 1,834 & 61,98 \% & 31,12 \% & 6,87 \% \\ \text { Implícito I } & 1,3 & 1,3 & 1 & 1 & 0 & 43,40 \% & 27,96 \% & 28,64 \% \\ \text { Implícito II } & 1,3 & 1,207 & 1 & 0,907 & 0,093 & 42,95 \% & 29,38 \% & 27,67 \%\end{array}$

Implícito II

$\begin{array}{llll}1,3 & 1,207 & 1 & 0,907\end{array}$

$0,093 \quad 42,95 \% \quad 29,38 \% \quad 27,67 \%$

Flamengo 0x1 Bragantino

$\begin{array}{lrrrrrrrr}\text { SD 0 } & 2,298 & 2,298 & 0,863 & 0,863 & 0 & 69,62 \% & 17,85 \% & 12,52 \% \\ \text { SD I } & 2,298 & 2,3 & 0,863 & 0,865 & -0,002 & & & \\ \text { "Chance" I } & 2,413 & 2,413 & 0,77 & 0,77 & 0 & 73,72 \% & 16,26 \% & 10,02 \% \\ \text { "Chance" II } & 4,07 & 2,236 & 2,696 & 0,862 & 1,834 & 68,21 \% & 18,42 \% & 13,04 \% \\ \text { Implícito I } & 2 & 2 & 0,7 & 0,7 & 0 & 67,95 \% & 19,98 \% & 12,07 \% \\ \text { Implícito II } & 2 & 1,708 & 0,7 & 0,408 & 0,292 & 69,69 \% & 22,03 \% & 8,28 \%\end{array}$

Implícito II

0,292

$69,69 \% 22,03 \%$

$8,28 \%$

Juventude 0x0 Botafogo

$\begin{array}{lrrrrrrrr}\text { SD 0 } & 1,298 & 1,298 & 0,933 & 0,933 & 0 & 44,95 \% & 28,26 \% & 26,79 \% \\ \text { SD I } & 1,298 & 1,006 & 0,933 & 0,641 & 0,292 & 43,34 \% & 33,84 \% & 22,83 \% \\ \text { "Chance" I } & 1,214 & 1,214 & 0,957 & 0,957 & 0 & 41,94 \% & 29,04 \% & 29,01 \% \\ \text { "Chance" II } & 3,091 & 1,257 & 2,798 & 0,964 & 1,834 & 42,99 \% & 28,56 \% & 28,40 \% \\ \text { Implícito I } & 1,9 & 1,9 & 1,4 & 1,4 & 0 & 49,28 \% & 22,31 \% & 28,41 \% \\ \text { Implícito II } & 1,9 & 0,903 & 1,4 & 0,403 & 0,997 & 46,25 \% & 37,90 \% & 15,85 \%\end{array}$

Implícito II

$1,90,903 \quad 1,4 \quad 0,403$

0,997

$46,25 \% \quad 37,90 \% \quad 15,85 \%$

América RN 2x2 Portuguesa

$\begin{array}{lrrrr}\text { SD } 0 & 1,371 & 1,371 & 1,563 & 1,563 \\ \text { SD I } & 1,371 & 1,146 & 1,563 & 1,338 \\ \text { "Chance" I } & 1,259 & 1,259 & 1,517 & 1,517 \\ \text { "Chance" II } & 3,127 & 1,293 & 3,45 & 1,616 \\ \text { Implícito I } & 1 & 1 & 1,5 & 1,5 \\ \text { Implícito II } & 1 & 0,803 & 1,5 & 1,303\end{array}$

$\begin{array}{rrrr}0 & 33,50 \% & 24,49 \% & 42,01 \% \\ 0,225 & 31,95 \% & 26,95 \% & 41,11 \% \\ 0 & 31,57 \% & 25,17 \% & 43,27 \% \\ 1,834 & 30,59 \% & 24,38 \% & 44,90 \% \\ 0 & 25,22 \% & 25,98 \% & 48,79 \% \\ 0,197 & 23,00 \% & 28,66 \% & 48,34 \%\end{array}$

Vitória 1x3 Santos

$\begin{array}{lrrrrrrrr}\text { SD 0 } & 1,85 & 1,85 & 1,581 & 1,581 & 0 & 44,39 \% & 22,33 \% & 33,28 \% \\ \text { SD I } & 1,85 & 2,623 & 1,581 & 2,354 & -0,773 & & & \\ \text { "Chance" I } & 1,864 & 1,864 & 1,586 & 1,586 & 0 & 44,60 \% & 22,25 \% & 33,16 \% \\ \text { "Chance" II } & 3,696 & 1,862 & 3,496 & 1,662 & 1,834 & 42,89 \% & 22,09 \% & 34,78 \% \\ \text { Implícito I } & 1 & 1 & 1,5 & 1,5 & 0 & 25,22 \% & 25,98 \% & 48,79 \% \\ \text { Implícito II } & 1 & 0,908 & 1,5 & 1,408 & 0,092 & 24,26 \% & 27,13 \% & 48,61 \%\end{array}$


Guarani 1x2 América MG

$\begin{array}{lrrrrrrrr}\text { SD 0 } & 2,236 & 2,236 & 0,815 & 0,815 & 0 & 69,63 \% & 18,17 \% & 12,19 \% \\ \text { SD I } & 2,236 & 1,598 & 0,815 & 0,177 & 0,638 & 74,15 \% & 22,09 \% & 3,76 \% \\ \text { "Chance" I } & 2,196 & 2,196 & 0,853 & 0,853 & 0 & 68,00 \% & 18,75 \% & 13,24 \% \\ \text { "Chance" II } & 3,989 & 2,155 & 2,637 & 0,802 & 1,834 & 68,15 \% & 18,90 \% & 12,67 \% \\ \text { Implícito I } & 2,1 & 2,1 & 0,7 & 0,7 & 0 & 69,89 \% & 18,89 \% & 11,21 \% \\ \text { Implícito II } & 2,1 & 1,603 & 0,7 & 0,203 & 0,497 & 73,46 \% & 22,23 \% & 4,30 \%\end{array}$

Internacional 2x1 Paraná

$\begin{array}{lrrrrrrrr}\text { SD 0 } & 1,767 & 1,767 & 0,395 & 0,395 & 0 & 71,33 \% & 21,06 \% & 7,61 \% \\ \text { SD I } & 1,767 & 1,243 & 0,395 & -0,129 & 0,524 & & & \\ \text { "Chance" I } & 1,697 & 1,697 & 0,606 & 0,606 & 0 & 63,90 \% & 23,24 \% & 12,85 \% \\ \text { "Chance" II } & 3,514 & 1,68 & 2,23 & 0,396 & 1,834 & 69,30 \% & 22,39 \% & 8,20 \% \\ \text { Implícito I } & 1,7 & 1,7 & 0,9 & 0,9 & 0 & 56,26 \% & 23,95 \% & 19,78 \% \\ \text { Implícito II } & 1,7 & 1,307 & 0,9 & 0,507 & 0,393 & 56,58 \% & 29,02 \% & 14,40 \%\end{array}$

São Paulo 0x2 Cruzeiro

$\begin{array}{lrrrrrrrr}\text { SD 0 } & 1,846 & 1,846 & 0,673 & 0,673 & 0 & 65,45 \% & 21,63 \% & 12,92 \% \\ \text { SD I } & 1,846 & 1,23 & 0,673 & 0,057 & 0,616 & 68,74 \% & 29,58 \% & 1,68 \% \\ \text { "Chance" I } & 1,716 & 1,716 & 0,765 & 0,765 & 0 & 60,10 \% & 23,52 \% & 16,38 \% \\ \text { "Chance" II } & 3,562 & 1,728 & 2,47 & 0,635 & 1,834 & 63,70 \% & 22,95 \% & 13,23 \% \\ \text { Implícito I } & 2 & 2 & 1 & 1 & 0 & 60,57 \% & 21,17 \% & 18,26 \% \\ \text { Implícito II } & 2 & 1,408 & 1 & 0,408 & 0,592 & 62,35 \% & 27,04 \% & 10,60 \%\end{array}$

Atlético PR 0x0 Grêmio

\begin{tabular}{|c|c|c|c|c|c|c|c|c|}
\hline SD 0 & 2,267 & 2,267 & 1,007 & 1,007 & 0 & $65,74 \%$ & $18,81 \%$ & $15,45 \%$ \\
\hline SD I & 2,267 & 2,265 & 1,007 & 1,005 & 0,002 & $65,75 \%$ & $18,82 \%$ & $15,44 \%$ \\
\hline "Chance" I & 2,222 & 2,222 & 0,998 & 0,998 & 0 & $65,09 \%$ & $19,16 \%$ & $15,74 \%$ \\
\hline "Chance" II & 4,006 & 2,172 & 2,8 & 0,966 & 1,834 & $64,59 \%$ & $19,48 \%$ & $15,64 \%$ \\
\hline Implícito I & 1,4 & 1,4 & 0,9 & 0,9 & 0 & $48,59 \%$ & $27,24 \%$ & $24,17 \%$ \\
\hline Implícito II & 1,4 & $-1,162$ & 0,9 & $-1,662$ & 2,562 & & & \\
\hline
\end{tabular}

Goiás 2x0 sport

$\begin{array}{lrrrrrrrr}\text { SD 0 } & 1,432 & 1,432 & 1,32 & 1,32 & 0 & 39,81 \% & 25,49 \% & 34,70 \% \\ \text { SD I } & 1,432 & 1,993 & 1,32 & 1,881 & -0,561 & & & \\ \text { "Chance" I } & 1,358 & 1,358 & 1,35 & 1,35 & 0 & 37,29 \% & 25,77 \% & 36,94 \% \\ \text { "Chance" II } & 3,237 & 1,403 & 3,247 & 1,412 & 1,834 & 37,13 \% & 25,20 \% & 37,57 \% \\ \text { Implícito I } & 1,3 & 1,3 & 1,3 & 1,3 & 0 & 36,80 \% & 26,39 \% & 36,80 \% \\ \text { Implícito II } & 1,3 & 1,003 & 1,3 & 1,003 & 0,297 & 34,60 \% & 30,80 \% & 34,60 \%\end{array}$

Ponte Preta 0x1 Flamengo

$\begin{array}{lrrrrrrrr}\text { SD 0 } & 1,405 & 1,405 & 1,246 & 1,246 & 0 & 40,69 \% & 26,00 \% & 33,31 \% \\ \text { SD I } & 1,405 & 0,444 & 1,246 & 0,285 & 0,961 & 28,57 \% & 54,54 \% & 16,89 \% \\ \text { "Chance" I } & 1,156 & 1,156 & 1,257 & 1,257 & 0 & 33,80 \% & 27,53 \% & 38,67 \% \\ \text { "Chance" II } & 2,865 & 1,298 & 2,804 & 1,236 & 1,567 & 38,05 \% & 26,77 \% & 35,14 \% \\ \text { Implícito I } & 1 & 1 & 1,5 & 1,5 & 0 & 25,22 \% & 25,98 \% & 48,79 \% \\ \text { Implícito II } & 1 & 0,707 & 1,5 & 1,207 & 0,293 & 21,69 \% & 30,28 \% & 48,02 \%\end{array}$


Botafogo 3xo São Paulo

$\begin{array}{lrrrrrrrr}\text { SD 0 } & 1,546 & 1,546 & 1,252 & 1,252 & 0 & 44,15 \% & 24,98 \% & 30,86 \% \\ \text { SD I } & 1,546 & 1,077 & 1,252 & 0,783 & 0,469 & 42,03 \% & 31,73 \% & 26,24 \% \\ \text { "Chance" I } & 1,423 & 1,423 & 1,196 & 1,196 & 0 & 42,25 \% & 26,08 \% & 31,68 \% \\ \text { "Chance" II } & 3,019 & 1,452 & 2,798 & 1,231 & 1,567 & 42,19 \% & 25,73 \% & 32,04 \% \\ \text { Implícito I } & 1,5 & 1,5 & 1,6 & 1,6 & 0 & 35,92 \% & 23,82 \% & 40,26 \% \\ \text { Implícito II } & 1,5 & -1,495 & 1,6 & -1,395 & 2,995 & & & \end{array}$

Palmeiras 1x2 Sport

$\begin{array}{lrrrrrrrr}\text { SD 0 } & 1,979 & 1,979 & 0,801 & 0,801 & 0 & 64,99 \% & 20,69 \% & 14,32 \% \\ \text { SD I } & 1,979 & 2,557 & 0,801 & 1,379 & -0,578 & & & \\ \text { "Chance" I } & 2,047 & 2,047 & 0,874 & 0,874 & 0 & 64,59 \% & 20,30 \% & 15,11 \% \\ \text { "Chance" II } & 3,509 & 1,942 & 2,418 & 0,85 & 1,567 & 62,87 \% & 21,28 \% & 15,74 \% \\ \text { Implícito I } & 1,5 & 1,5 & 1,4 & 1,4 & 0 & 39,86 \% & 24,74 \% & 35,40 \% \\ \text { Implícito II } & 1,5 & 0,903 & 1,4 & 0,803 & 0,597 & 35,83 \% & 33,92 \% & 30,25 \%\end{array}$

Implícito II $\quad 1,5 \quad 0,903 \quad 1,4 \quad 0,803 \quad 0,597 \quad 35,83 \% \quad 33,92 \% 30,25 \%$

Coritiba 0xo Corinthians

$\begin{array}{lrrrrrrrr}\text { SD 0 } & 1,184 & 1,184 & 1,293 & 1,293 & 0 & 33,84 \% & 27,10 \% & 39,06 \% \\ \text { SD I } & 1,184 & 1,416 & 1,293 & 1,525 & -0,232 & & & \\ \text { "Chance" I } & 1,06 & 1,06 & 1,23 & 1,23 & 0 & 31,65 \% & 28,31 \% & 40,04 \% \\ \text { "Chance" II } & 2,647 & 1,08 & 2,814 & 1,246 & 1,567 & 31,87 \% & 28,06 \% & 40,05 \% \\ \text { Implícito I } & 0,8 & 0,8 & 2 & 2 & 0 & 14,09 \% & 20,47 \% & 65,44 \% \\ \text { Implícito II } & 0,8 & 0,526 & 2 & 1,726 & 0,274 & 10,76 \% & 22,47 \% & 66,77 \%\end{array}$

$\begin{array}{llllllll}\text { Implícito II } & 0,8 & 0,526 & 2 & 1,726 & 0,274 & 10,76 \% & 22,47 \%\end{array}$

Vitória $3 \times 3$ Guarani

$\begin{array}{lrrrrrrrr}\text { SD 0 } & 1,895 & 1,895 & 1,454 & 1,454 & 0 & 48,02 \% & 22,29 \% & 29,69 \% \\ \text { SD I } & 1,895 & 2,046 & 1,454 & 1,604 & -0,15 & & & \\ \text { "Chance" I } & 1,897 & 1,897 & 1,407 & 1,407 & 0 & 49,06 \% & 22,32 \% & 28,62 \% \\ \text { "Chance" II } & 3,437 & 1,87 & 3,072 & 1,504 & 1,567 & 46,31 \% & 22,37 \% & 31,21 \% \\ \text { Implícito I } & 1,4 & 1,4 & 1,5 & 1,5 & 0 & 35,40 \% & 24,74 \% & 39,86 \% \\ \text { Implícito II } & 1,4 & 0,707 & 1,5 & 0,807 & 0,693 & 28,85 \% & 36,46 \% & 34,69 \%\end{array}$

Implícito II $\quad 1,4 \quad 0,707 \quad 1,5 \quad 0,807 \quad 0,693 \quad 28,85 \% \quad 36,46 \%$

América MG 3xo Paraná

$\begin{array}{lrrrrrrrr}\text { SD 0 } & 1,618 & 1,618 & 1,237 & 1,237 & 0 & 46,27 \% & 24,49 \% & 29,24 \% \\ \text { SD I } & 1,618 & 1,151 & 1,237 & 0,77 & 0,467 & 44,67 \% & 30,81 \% & 24,52 \% \\ \text { "Chance" I } & 1,466 & 1,466 & 1,064 & 1,064 & 0 & 46,38 \% & 26,16 \% & 27,46 \% \\ \text { "Chance" II } & 3,057 & 1,489 & 2,763 & 1,195 & 1,567 & 43,95 \% & 25,58 \% & 30,43 \% \\ \text { Implícito I } & 1,5 & 1,5 & 1 & 1 & 0 & 48,79 \% & 25,98 \% & 25,22 \% \\ \text { Implícito II } & 1,5 & 1,207 & 1 & 0,707 & 0,293 & 48,02 \% & 30,28 \% & 21,69 \%\end{array}$

Goiás 0xo Cruzeiro

$\begin{array}{lrrrrrrrr}\text { SD 0 } & 1,155 & 1,155 & 0,964 & 0,964 & 0 & 40,07 \% & 29,61 \% & 30,32 \% \\ \text { SD I } & 1,155 & 1,015 & 0,964 & 0,824 & 0,14 & 39,04 \% & 32,26 \% & 28,70 \% \\ \text { "Chance" I } & 1,082 & 1,082 & 0,99 & 0,99 & 0 & 37,29 \% & 30,15 \% & 32,56 \% \\ \text { "Chance" II } & 2,671 & 1,103 & 2,538 & 0,97 & 1,567 & 38,37 \% & 30,09 \% & 31,52 \% \\ \text { Implícito I } & 1,4 & 1,4 & 1,8 & 1,8 & 0 & 30,03 \% & 22,95 \% & 47,02 \% \\ \text { Implícito II } & 1,4 & 0,608 & 1,8 & 1,008 & 0,792 & 21,67 \% & 34,05 \% & 44,28 \%\end{array}$


Grêmio 0x0 Juventude

$\begin{array}{lrrrrrrrr}\text { SD 0 } & 1,111 & 1,111 & 1,336 & 1,336 & 0 & 31,03 \% & 27,13 \% & 41,83 \% \\ \text { SD I } & 1,111 & 0,351 & 1,336 & 0,575 & 0,761 & 18,26 \% & 48,02 \% & 33,72 \% \\ \text { "Chance" I } & 1,041 & 1,041 & 1,223 & 1,223 & 0 & 31,23 \% & 28,48 \% & 40,29 \% \\ \text { "Chance" II } & 2,651 & 1,084 & 2,958 & 1,391 & 1,567 & 29,29 \% & 26,77 \% & 43,90 \% \\ \text { Implícito I } & 1 & 1 & 1,3 & 1,3 & 0 & 28,64 \% & 27,96 \% & 43,40 \% \\ \text { Implícito II } & 1 & 1,003 & 1,3 & 1,303 & -0,003 & & & \end{array}$

América RN 0x0 Internacional

$\begin{array}{lrrrrrrrr}\text { SD 0 } & 1,221 & 1,221 & 1,549 & 1,549 & 0 & 30,03 \% & 25,05 \% & 44,92 \% \\ \text { SD I } & 1,221 & 1,068 & 1,549 & 1,396 & 0,153 & 28,78 \% & 26,79 \% & 44,44 \% \\ \text { "Chance" I } & 1,108 & 1,108 & 1,543 & 1,543 & 0 & 27,29 \% & 25,37 \% & 47,34 \% \\ \text { "Chance" II } & 2,712 & 1,145 & 3,222 & 1,654 & 1,567 & 26,38 \% & 24,36 \% & 49,19 \% \\ \text { Implícito I } & 1 & 1 & 2 & 2 & 0 & 18,26 \% & 21,17 \% & 60,57 \% \\ \text { Implícito II } & 1 & 0,408 & 2 & 1,408 & 0,592 & 10,60 \% & 27,04 \% & 62,35 \%\end{array}$

Implícito II

Corinthians 1xo Botafogo

$\begin{array}{lrrrrrrrr}\text { SD 0 } & 1,405 & 1,405 & 1,019 & 1,019 & 0 & 45,83 \% & 26,87 \% & 27,31 \% \\ \text { SD I } & 1,405 & 1,325 & 1,019 & 0,939 & 0,08 & 45,56 \% & 27,95 \% & 26,49 \% \\ \text { "Chance" I } & 1,337 & 1,337 & 1,008 & 1,008 & 0 & 44,24 \% & 27,57 \% & 28,19 \% \\ \text { "Chance" II } & 3,077 & 1,35 & 2,744 & 1,017 & 1,727 & 44,34 \% & 27,40 \% & 28,21 \% \\ \text { Implícito I } & 1,9 & 1,9 & 1,4 & 1,4 & 0 & 49,28 \% & 22,31 \% & 28,41 \% \\ \text { Implícito II } & 1,9 & 0,903 & 1,4 & 0,403 & 0,997 & 46,25 \% & 37,90 \% & 15,85 \%\end{array}$

Implícito II $\quad 1,9 \quad 0,903 \quad 1,4 \quad 0,403$

Santos 1x0 Palmeiras

$\begin{array}{lrrrrrrrr}\text { SD 0 } & 1,424 & 1,424 & 1,229 & 1,229 & 0 & 41,55 \% & 25,94 \% & 32,51 \% \\ \text { SD I } & 1,424 & 2,007 & 1,229 & 1,812 & -0,583 & & & \\ \text { "Chance" I } & 1,333 & 1,333 & 1,237 & 1,237 & 0 & 38,99 \% & 26,54 \% & 34,47 \% \\ \text { "Chance" II } & 3,131 & 1,404 & 3,018 & 1,291 & 1,727 & 39,65 \% & 25,80 \% & 34,48 \% \\ \text { Implícito I } & 1,5 & 1,5 & 1,4 & 1,4 & 0 & 39,86 \% & 24,74 \% & 35,40 \% \\ \text { Implícito II } & 1,5 & 0,903 & 1,4 & 0,803 & 0,597 & 35,83 \% & 33,92 \% & 30,25 \%\end{array}$

Implícito II $\quad 1,5 \quad 0,903 \quad 1,4 \quad 0,803 \quad 0,597 \quad 35,83 \% \quad 33,92 \% \quad 30,25 \%$

Vasco 2x1 Guarani

$\begin{array}{lrrrrrrrr}\text { SD 0 } & 1,878 & 1,878 & 0,824 & 0,824 & 0 & 62,26 \% & 21,86 \% & 15,88 \% \\ \text { SD I } & 1,878 & 1,12 & 0,824 & 0,066 & 0,758 & 64,99 \% & 32,83 \% & 2,18 \% \\ \text { "Chance" I } & 1,929 & 1,929 & 0,894 & 0,894 & 0 & 61,62 \% & 21,55 \% & 16,82 \% \\ \text { "Chance" II } & 3,589 & 1,862 & 2,592 & 0,865 & 1,727 & 60,75 \% & 22,16 \% & 16,96 \% \\ \text { Implícito I } & 2 & 2 & 1,3 & 1,3 & 0 & 53,67 \% & 21,64 \% & 24,70 \% \\ \text { Implícito II } & 2 & 1,108 & 1,3 & 0,408 & 0,892 & 53,36 \% & 33,07 \% & 13,57 \%\end{array}$

Grêmio 2×2 Flamengo

\begin{tabular}{|c|c|c|c|c|c|c|c|c|}
\hline $\mathrm{SD} 0$ & 1,659 & 1,659 & 1,639 & 1,639 & 0 & $38,91 \%$ & $23,03 \%$ & $38,06 \%$ \\
\hline SD I & 1,659 & 1,124 & 1,639 & 1,104 & 0,535 & $36,05 \%$ & $28,91 \%$ & $35,04 \%$ \\
\hline "Chance" I & 1,525 & 1,525 & 1,545 & 1,545 & 0 & $37,58 \%$ & $23,98 \%$ & $38,44 \%$ \\
\hline "Chance" II & 3,322 & 1,595 & 3,371 & 1,644 & 1,727 & $37,28 \%$ & $23,26 \%$ & $39,33 \%$ \\
\hline Implícito I & 1 & 1 & 1 & 1 & 0 & $34,57 \%$ & $30,85 \%$ & $34,57 \%$ \\
\hline Implícito II & 1 & 1,303 & 1 & 1,303 & $-0,303$ & & & \\
\hline
\end{tabular}


Sport 1x0 São Paulo

$\begin{array}{lrrrrrrrr}\text { SD 0 } & 1,69 & 1,69 & 1,379 & 1,379 & 0 & 44,90 \% & 23,68 \% & 31,41 \% \\ \text { SD I } & 1,69 & 2,268 & 1,379 & 1,957 & -0,578 & & & \\ \text { "Chance" I } & 1,647 & 1,647 & 1,331 & 1,331 & 0 & 44,90 \% & 24,08 \% & 31,03 \% \\ \text { "Chance" II } & 3,384 & 1,657 & 3,106 & 1,379 & 1,727 & 44,04 \% & 23,89 \% & 31,96 \% \\ \text { Implícito I } & 1,9 & 1,9 & 1,3 & 1,3 & 0 & 51,47 \% & 22,36 \% & 26,17 \% \\ \text { Implícito II } & 1,9 & -1,195 & 1,3 & -1,795 & 3,095 & & & \end{array}$

Portuguesa 0x1 Vitória

$\begin{array}{lrrrrrrrr}\text { SD 0 } & 2,246 & 2,246 & 1,424 & 1,424 & 0 & 56,09 \% & 20,05 \% & 23,85 \% \\ \text { SD I } & 2,246 & 2,563 & 1,424 & 1,741 & -0,317 & & & \\ \text { "Chance" I } & 2,435 & 2,435 & 1,31 & 1,31 & 0 & 62,18 \% & 18,53 \% & 19,29 \% \\ \text { "Chance" II } & 3,952 & 2,225 & 3,134 & 1,407 & 1,727 & 55,77 \% & 20,17 \% & 23,77 \% \\ \text { Implícito I } & 1,8 & 1,8 & 1,3 & 1,3 & 0 & 49,19 \% & 23,07 \% & 27,73 \% \\ \text { Implícito II } & 1,8 & 0,907 & 1,3 & 0,407 & 0,893 & 46,29 \% & 37,74 \% & 15,97 \%\end{array}$

Bragantino $1 \times 2$ Coritiba

$\begin{array}{lrrrrrrrr}\text { SD 0 } & 1,232 & 1,232 & 1,121 & 1,121 & 0 & 38,75 \% & 27,93 \% & 33,32 \% \\ \text { SD I } & 1,232 & 0,999 & 1,121 & 0,888 & 0,233 & 37,04 \% & 31,88 \% & 31,09 \% \\ \text { "Chance" I } & 1,013 & 1,013 & 1,037 & 1,037 & 0 & 34,18 \% & 30,38 \% & 35,43 \% \\ \text { "Chance" II } & 2,865 & 1,138 & 2,839 & 1,112 & 1,727 & 36,25 \% & 28,74 \% & 34,97 \% \\ \text { Implícito I } & 1 & 1 & 1,5 & 1,5 & 0 & 25,22 \% & 25,98 \% & 48,79 \% \\ \text { Implícito II } & 1 & 0,62 & 1,5 & 1,12 & 0,38 & 20,32 \% & 32,02 \% & 47,66 \%\end{array}$

Paraná 1xo Goiás

$\begin{array}{lrrrrrrrr}\text { SD 0 } & 1,192 & 1,192 & 0,948 & 0,948 & 0 & 41,53 \% & 29,32 \% & 29,14 \% \\ \text { SD I } & 1,192 & 1,163 & 0,948 & 0,919 & 0,029 & 41,36 \% & 29,81 \% & 28,83 \% \\ \text { "Chance" I } & 1,094 & 1,094 & 0,966 & 0,966 & 0 & 38,19 \% & 30,22 \% & 31,59 \% \\ \text { "Chance" II } & 2,863 & 1,136 & 2,683 & 0,955 & 1,727 & 39,69 \% & 29,86 \% & 30,42 \% \\ \text { Implícito I } & 1,4 & 1,4 & 1,8 & 1,8 & 0 & 30,03 \% & 22,95 \% & 47,02 \% \\ \text { Implícito II } & 1,4 & 0,503 & 1,8 & 0,903 & 0,897 & 19,60 \% & 36,99 \% & 43,41 \%\end{array}$

$\begin{array}{llllllll}\text { Implícito II } \quad 1,4 & 0,503 & 1,8 & 0,903 & 0,897 & 19,60 \% & 36,99 \% & 43,41 \%\end{array}$

Cruzeiro 0x2 América RN

$\begin{array}{lrrrrrrrr}\text { SD 0 } & 1,8 & 1,8 & 0,609 & 0,609 & 0 & 66,14 \% & 21,90 \% & 11,96 \% \\ \text { SD I } & 1,8 & 1,255 & 0,609 & 0,065 & 0,544 & 69,23 \% & 28,92 \% & 1,85 \% \\ \text { "Chance" I } & 1,78 & 1,78 & 0,725 & 0,725 & 0 & 62,62 \% & 22,64 \% & 14,74 \% \\ \text { "Chance" II } & 3,529 & 1,802 & 2,302 & 0,575 & 1,727 & 67,01 \% & 21,69 \% & 11,19 \% \\ \text { Implícito I } & 2 & 2 & 1 & 1 & 0 & 60,57 \% & 21,17 \% & 18,26 \% \\ \text { Implícito II } & 2 & 1,303 & 1 & 0,303 & 0,697 & 62,67 \% & 28,82 \% & 8,51 \%\end{array}$

Juventude 0x0 Atlético MG

$\begin{array}{lrrrrrrrr}\text { SD 0 } & 1,458 & 1,458 & 0,848 & 0,848 & 0 & 51,47 \% & 26,66 \% & 21,87 \% \\ \text { SD I } & 1,458 & 1,096 & 0,848 & 0,486 & 0,362 & 50,65 \% & 33,05 \% & 16,29 \% \\ \text { "Chance" I } & 1,364 & 1,364 & 0,867 & 0,867 & 0 & 48,42 \% & 27,74 \% & 23,85 \% \\ \text { "Chance" II } & 3,156 & 1,429 & 2,583 & 0,856 & 1,727 & 50,43 \% & 26,99 \% & 22,52 \% \\ \text { Implícito I } & 1,6 & 1,6 & 1,4 & 1,4 & 0 & 42,31 \% & 24,17 \% & 33,52 \% \\ \text { Implícito II } & 1,6 & 0,72 & 1,4 & 0,52 & 0,88 & 35,85 \% & 40,82 \% & 23,33 \%\end{array}$


América MG 0x1 Internacional

\begin{tabular}{|c|c|c|c|c|c|c|c|c|}
\hline $\mathrm{SD} \quad 0$ & 1,222 & 1,222 & 1,486 & 1,486 & 0 & $31,18 \%$ & $25,51 \%$ & $43,30 \%$ \\
\hline SD I & 1,222 & 0,655 & 1,486 & 0,919 & 0,567 & $24,83 \%$ & $35,19 \%$ & $39,98 \%$ \\
\hline "Chance" I & 1,084 & 1,084 & 1,367 & 1,367 & 0 & $29,74 \%$ & $26,98 \%$ & $43,27 \%$ \\
\hline "Chance" II & 2,877 & 1,15 & 3,207 & 1,48 & 1,727 & $29,43 \%$ & $25,80 \%$ & $44,70 \%$ \\
\hline Implícito I & 1 & 1 & 1,1 & 1,1 & 0 & $32,48 \%$ & $29,91 \%$ & $37,61 \%$ \\
\hline Implícito II & 1 & 1,203 & 1,1 & 1,303 & $-0,203$ & & & \\
\hline
\end{tabular}

Santos $4 \times 4$ Atlético MG

$\begin{array}{lrrrrrrrr}\text { SD 0 } & 1,941 & 1,941 & 0,994 & 0,994 & 0 & 59,45 \% & 21,71 \% & 18,84 \% \\ \text { SD I } & 1,941 & 2,212 & 0,994 & 1,265 & -0,271 & & & \\ \text { "Chance" I } & 2,471 & 2,471 & 1,305 & 1,305 & 0 & 62,94 \% & 18,26 \% & 18,79 \% \\ \text { "Chance" II } & 3,51 & 1,902 & 2,629 & 1,021 & 1,608 & 57,84 \% & 22,13 \% & 19,92 \% \\ \text { Implícito I } & 2 & 2 & 1 & 1 & 0 & 60,57 \% & 21,17 \% & 18,26 \% \\ \text { Implícito II } & 2 & 1,303 & 1 & 0,303 & 0,697 & 62,67 \% & 28,82 \% & 8,51 \%\end{array}$

Guarani 2x1 América RN

$\begin{array}{lrrrrrrrr}\text { SD 0 } & 1,907 & 1,907 & 1,037 & 1,037 & 0 & 57,67 \% & 22,11 \% & 20,22 \% \\ \text { SD I } & 1,907 & 1,781 & 1,037 & 0,911 & 0,126 & 57,90 \% & 23,12 \% & 18,99 \% \\ \text { "Chance" I } & 2,574 & 2,574 & 1,383 & 1,383 & 0 & 63,17 \% & 17,79 \% & 19,03 \% \\ \text { "Chance" II } & 3,496 & 1,888 & 2,522 & 0,914 & 1,608 & 60,15 \% & 22,02 \% & 17,72 \% \\ \text { Implícito I } & 1,6 & 1,6 & 1,4 & 1,4 & 0 & 42,31 \% & 24,17 \% & 33,52 \% \\ \text { Implícito II } & 1,6 & 1,008 & 1,4 & 0,808 & 0,592 & 39,20 \% & 32,47 \% & 28,33 \%\end{array}$

Implícito II $\quad 1,6 \quad 1,008 \quad 1,4 \quad 0,808 \quad 0,592 \quad 39,20 \% \quad 32,47 \% \quad 28,33 \%$

Ponte Preta 2x0 Atlético PR

\begin{tabular}{|c|c|c|c|c|c|c|c|c|}
\hline SD 0 & 0,921 & 0,921 & 1,002 & 1,002 & 0 & $32,07 \%$ & $31,56 \%$ & $36,38 \%$ \\
\hline SD I & 0,921 & 0,347 & 1,002 & 0,428 & 0,574 & $20,48 \%$ & $53,17 \%$ & $26,35 \%$ \\
\hline "Chance" I & 1,353 & 1,353 & 2,89 & 2,89 & 0 & $15,33 \%$ & $15,62 \%$ & $69,03 \%$ \\
\hline "Chance" II & 2,413 & 0,806 & 2,541 & 0,933 & 1,608 & $29,72 \%$ & $33,49 \%$ & $36,78 \%$ \\
\hline Implícito I & 1 & 1 & 1,1 & 1,1 & 0 & $32,48 \%$ & $29,91 \%$ & $37,61 \%$ \\
\hline Implícito II & 1 & 1,107 & 1,1 & 1,207 & $-0,107$ & & & \\
\hline
\end{tabular}

Paraná 2x2 Bragantino

$\begin{array}{lrrrrrrrr}\text { SD 0 } & 1,648 & 1,648 & 0,667 & 0,667 & 0 & 61,08 \% & 24,11 \% & 14,81 \% \\ \text { SD I } & 1,648 & 1,604 & 0,667 & 0,623 & 0,044 & 61,21 \% & 24,57 \% & 14,22 \% \\ \text { "Chance" I } & 2,724 & 2,724 & 1,385 & 1,385 & 0 & 65,70 \% & 16,81 \% & 17,48 \% \\ \text { "Chance" II } & 3,166 & 1,558 & 2,239 & 0,631 & 1,608 & 59,78 \% & 25,24 \% & 14,93 \% \\ \text { Implícito I } & 1,2 & 1,2 & 0,9 & 0,9 & 0 & 42,92 \% & 29,50 \% & 27,58 \% \\ \text { Implícito II } & 1,2 & 1,22 & 0,9 & 0,92 & -0,02 & & & \end{array}$

Botafogo 1x1 Cruzeiro

$\begin{array}{lrrrrrrrr}\text { SD 0 } & 1,466 & 1,466 & 0,674 & 0,674 & 0 & 56,28 \% & 26,60 \% & 17,12 \% \\ \text { SD I } & 1,466 & 0,89 & 0,674 & 0,098 & 0,576 & 55,45 \% & 40,55 \% & 4,00 \% \\ \text { "Chance" I } & 1,36 & 1,36 & 0,779 & 0,779 & 0 & 50,58 \% & 27,98 \% & 21,45 \% \\ \text { "Chance" II } & 2,994 & 1,398 & 2,256 & 0,66 & 1,596 & 54,80 \% & 27,56 \% & 17,61 \% \\ \text { Implícito I } & 1 & 1 & 1,4 & 1,4 & 0 & 26,88 \% & 26,97 \% & 46,15 \% \\ \text { Implícito II } & 1 & 0,903 & 1,4 & 1,303 & 0,097 & 25,85 \% & 28,33 \% & 45,82 \%\end{array}$


Internacional $1 \times 1$ Corinthians

$\begin{array}{lrrrrrrrr}\text { SD 0 } & 1,358 & 1,358 & 0,699 & 0,699 & 0 & 52,65 \% & 28,12 \% & 19,23 \% \\ \text { SD I } & 1,358 & 1,32 & 0,699 & 0,661 & 0,038 & 52,60 \% & 28,72 \% & 18,68 \% \\ \text { "Chance" I } & 1,275 & 1,275 & 0,774 & 0,774 & 0 & 48,26 \% & 29,11 \% & 22,62 \% \\ \text { "Chance" II } & 2,912 & 1,316 & 2,312 & 0,716 & 1,596 & 50,97 \% & 28,69 \% & 20,32 \% \\ \text { Implícito I } & 1,4 & 1,4 & 2,1 & 2,1 & 0 & 25,39 \% & 21,00 \% & 53,61 \% \\ \text { Implícito II } & 1,4 & 0,203 & 2,1 & 0,903 & 1,197 & 8,11 \% & 39,45 \% & 52,43 \%\end{array}$

Flamengo 1x3 Juventude

$\begin{array}{lrrrrrrrr}\text { SD 0 } & 1,323 & 1,323 & 0,999 & 0,999 & 0 & 44,06 \% & 27,74 \% & 28,20 \% \\ \text { SD I } & 1,323 & 0,828 & 0,999 & 0,504 & 0,495 & 40,55 \% & 38,63 \% & 20,82 \% \\ \text { "Chance" I } & 1,2 & 1,2 & 0,948 & 0,948 & 0 & 41,75 \% & 29,24 \% & 29,01 \% \\ \text { "Chance" II } & 2,877 & 1,282 & 2,61 & 1,014 & 1,596 & 42,53 \% & 28,07 \% & 29,36 \% \\ \text { Implícito I } & 1,3 & 1,3 & 1,1 & 1,1 & 0 & 41,10 \% & 27,49 \% & 31,41 \% \\ \text { Implícito II } & 1,3 & 1,107 & 1,1 & 0,907 & 0,193 & 39,96 \% & 30,50 \% & 29,54 \%\end{array}$

Implícito II

$1,3 \quad 1,107 \quad 1,1 \quad 0,907$

$0,193 \quad 39,96 \% 30,50 \% \quad 29,54 \%$

América RN 1x2 Palmeiras

\begin{tabular}{|c|c|c|c|c|c|c|c|c|}
\hline $\mathrm{SD} 0$ & 1,115 & 1,115 & 1,627 & 1,627 & 0 & $26,09 \%$ & $24,64 \%$ & $49,27 \%$ \\
\hline SD I & 1,115 & 1,353 & 1,627 & 1,865 & $-0,238$ & & & \\
\hline "Chance" I & 1,037 & 1,037 & 1,655 & 1,655 & 0 & $23,74 \%$ & $24,45 \%$ & $51,81 \%$ \\
\hline "Chance" II & 2,644 & 1,049 & 3,3 & 1,704 & 1,596 & $23,28 \%$ & $23,99 \%$ & $52,66 \%$ \\
\hline Implícito I & 1 & 1 & 2,2 & 2,2 & 0 & $16,01 \%$ & $19,36 \%$ & $64,62 \%$ \\
\hline Implícito II & 1 & 0,208 & 2,2 & 1,408 & 0,792 & $5,27 \%$ & $26,13 \%$ & $68,59 \%$ \\
\hline Vitória 2x1 América & MG & & & & & & & \\
\hline SD 0 & 2,355 & 2,355 & 1,362 & 1,362 & 0 & $59,56 \%$ & $19,22 \%$ & $21,22 \%$ \\
\hline SD I & 2,355 & 2,761 & 1,362 & 1,768 & $-0,406$ & & & \\
\hline "Chance" I & 2,511 & 2,511 & 1,269 & 1,269 & 0 & $64,44 \%$ & $17,86 \%$ & $17,69 \%$ \\
\hline "Chance" II & 3,9 & 2,304 & 2,959 & 1,363 & 1,596 & $58,32 \%$ & $19,57 \%$ & $21,86 \%$ \\
\hline Implícito I & 1 & 1 & 1,1 & 1,1 & 0 & $32,48 \%$ & $29,91 \%$ & $37,61 \%$ \\
\hline Implícito II & 1 & 1,107 & 1,1 & 1,207 & $-0,107$ & & & \\
\hline
\end{tabular}

Atlético PR 2x2 Portuguesa

\begin{tabular}{|c|c|c|c|c|c|c|c|c|}
\hline SD 0 & 1,365 & 1,365 & 1,207 & 1,207 & 0 & $40,49 \%$ & $26,46 \%$ & $33,06 \%$ \\
\hline SD I & 1,365 & 1,204 & 1,207 & 1,046 & 0,161 & $39,62 \%$ & $28,63 \%$ & $31,75 \%$ \\
\hline "Chance" I & 1,231 & 1,231 & 1,166 & 1,166 & 0 & $37,74 \%$ & $27,67 \%$ & $34,59 \%$ \\
\hline "Chance" II & 2,854 & 1,258 & 2,772 & 1,176 & 1,596 & $38,24 \%$ & $27,40 \%$ & $34,32 \%$ \\
\hline Implícito I & 1 & 1 & 1,1 & 1,1 & 0 & $32,48 \%$ & $29,91 \%$ & $37,61 \%$ \\
\hline Implícito II & 1 & 1,107 & 1,1 & 1,207 & $-0,107$ & & & \\
\hline
\end{tabular}

Coritiba 0x0 sport

$\begin{array}{lrrrrrrrr}\text { SD 0 } & 1,283 & 1,283 & 1,401 & 1,401 & 0 & 34,35 \% & 25,85 \% & 39,80 \% \\ \text { SD I } & 1,283 & 1,554 & 1,401 & 1,672 & -0,271 & & & \\ \text { "Chance" I } & 1,195 & 1,195 & 1,373 & 1,373 & 0 & 32,60 \% & 26,46 \% & 40,94 \% \\ \text { "Chance" II } & 2,817 & 1,221 & 2,999 & 1,404 & 1,596 & 32,68 \% & 26,11 \% & 41,16 \% \\ \text { Implícito I } & 1 & 1 & 1 & 1 & 0 & 34,57 \% & 30,85 \% & 34,57 \% \\ \text { Implícito II } & 1 & 1,207 & 1 & 1,207 & -0,207 & & & \end{array}$


Goiás 0x0 Grêmio

$\begin{array}{lrrrrrrrr}\text { SD 0 } & 1,801 & 1,801 & 0,8 & 0,8 & 0 & 61,16 \% & 22,64 \% & 16,21 \% \\ \text { SD I } & 1,801 & 1,213 & 0,8 & 0,212 & 0,588 & 62,94 \% & 30,66 \% & 6,40 \% \\ \text { "Chance" I } & 1,686 & 1,686 & 0,885 & 0,885 & 0 & 56,31 \% & 24,08 \% & 19,61 \% \\ \text { "Chance" II } & 3,382 & 1,786 & 2,431 & 0,835 & 1,596 & 59,84 \% & 22,90 \% & 17,18 \% \\ \text { Implícito I } & 1,9 & 1,9 & 1 & 1 & 0 & 58,41 \% & 22,11 \% & 19,49 \% \\ \text { Implícito II } & 1,9 & 1,408 & 1 & 0,508 & 0,492 & 59,39 \% & 27,29 \% & 13,32 \%\end{array}$

São Paulo $1 \times 3$ Santos

$\begin{array}{lrrrrrrrr}\text { SD 0 } & 1,766 & 1,766 & 1,193 & 1,193 & 0 & 50,81 \% & 23,41 \% & 25,78 \% \\ \text { SD I } & 1,766 & 1,827 & 1,193 & 1,254 & -0,061 & & & \\ \text { "Chance" I } & 1,779 & 1,779 & 1,166 & 1,166 & 0 & 51,73 \% & 23,32 \% & 24,95 \% \\ \text { "Chance" II } & 3,338 & 1,742 & 2,814 & 1,218 & 1,596 & 49,62 \% & 23,58 \% & 26,72 \% \\ \text { Implícito I } & 1,3 & 1,3 & 1,5 & 1,5 & 0 & 32,90 \% & 25,14 \% & 41,96 \% \\ \text { Implícito II } & 1,3 & 1,026 & 1,5 & 1,226 & 0,274 & 30,75 \% & 28,54 \% & 40,70 \%\end{array}$

Implícito II $\quad 1,3 \quad 1,026 \quad 1,5 \quad 1,226 \quad 0,274 \quad 30,75 \% \quad 28,54 \% \quad 40,70 \%$

Atlético MG 0x2 Bragantino

$\begin{array}{lrrrrrrrr}\text { SD 0 } & 2,197 & 2,197 & 0,844 & 0,844 & 0 & 68,23 \% & 18,70 \% & 13,07 \% \\ \text { SD I } & 2,197 & 1,846 & 0,844 & 0,493 & 0,351 & 70,26 \% & 20,63 \% & 9,11 \% \\ \text { "Chance" I } & 2,254 & 2,254 & 0,84 & 0,84 & 0 & 69,36 \% & 18,14 \% & 12,50 \% \\ \text { "Chance" II } & 3,723 & 2,127 & 2,458 & 0,862 & 1,596 & 66,30 \% & 19,46 \% & 14,07 \% \\ \text { Implícito I } & 1,3 & 1,3 & 1 & 1 & 0 & 43,40 \% & 27,96 \% & 28,64 \% \\ \text { Implícito II } & 1,3 & 1,303 & 1 & 1,003 & -0,003 & & & \end{array}$

Corinthians 3x1 América MG

$\begin{array}{lrrrrrrrr}\text { SD 0 } & 1,932 & 1,932 & 0,69 & 0,69 & 0 & 66,82 \% & 20,70 \% & 12,48 \% \\ \text { SD I } & 1,932 & 2,055 & 0,69 & 0,813 & -0,123 & & & \\ \text { "Chance" I } & 1,896 & 1,896 & 0,779 & 0,779 & 0 & 63,79 \% & 21,50 \% & 14,70 \% \\ \text { "Chance" II } & 3,478 & 1,88 & 2,275 & 0,677 & 1,598 & 65,98 \% & 21,25 \% & 12,68 \% \\ \text { Implícito I } & 2 & 2 & 0,8 & 0,8 & 0 & 65,44 \% & 20,47 \% & 14,09 \% \\ \text { Implícito II } & 2 & 1,859 & 0,8 & 0,659 & 0,141 & 66,09 \% & 21,41 \% & 12,50 \%\end{array}$

Implícito II

$0,141 \quad 66,09 \% \quad 21,41 \% \quad 12,50 \%$

Bragantino 2x2 Botafogo

\begin{tabular}{|c|c|c|c|c|c|c|c|c|}
\hline SD 0 & 0,994 & 0,994 & 1,511 & 1,511 & 0 & $24,89 \%$ & $25,89 \%$ & $49,23 \%$ \\
\hline SD I & 0,994 & 0,915 & 1,511 & 1,432 & 0,079 & $24,06 \%$ & $26,86 \%$ & $49,08 \%$ \\
\hline "Chance" I & 0,939 & 0,939 & 1,43 & 1,43 & 0 & $24,74 \%$ & $26,83 \%$ & $48,44 \%$ \\
\hline "Chance" II & 2,555 & 0,957 & 3,105 & 1,507 & 1,598 & $23,99 \%$ & $25,99 \%$ & $49,98 \%$ \\
\hline Implícito I & 1 & 1 & 1,1 & 1,1 & 0 & $32,48 \%$ & $29,91 \%$ & $37,61 \%$ \\
\hline Implícito II & 1 & 1,107 & 1,1 & 1,207 & $-0,107$ & & & \\
\hline
\end{tabular}

Cruzeiro 2×2 Portuguesa

$\begin{array}{lrrrrrrrr}\text { SD 0 } & 1,346 & 1,346 & 0,919 & 0,919 & 0 & 46,63 \% & 27,79 \% & 25,58 \% \\ \text { SD I } & 1,346 & 0,88 & 0,919 & 0,453 & 0,466 & 43,95 \% & 37,98 \% & 18,08 \% \\ \text { "Chance" I } & 1,223 & 1,223 & 0,945 & 0,945 & 0 & 42,49 \% & 29,01 \% & 28,50 \% \\ \text { "Chance" II } & 2,851 & 1,253 & 2,523 & 0,924 & 1,598 & 43,85 \% & 28,79 \% & 27,33 \% \\ \text { Implícito I } & 1,5 & 1,5 & 1,1 & 1,1 & 0 & 46,42 \% & 25,77 \% & 27,81 \% \\ \text { Implícito II } & 1,5 & 1,203 & 1,1 & 0,803 & 0,297 & 45,41 \% & 29,95 \% & 24,64 \%\end{array}$


Flamengo 0x0 Atlético PR

$\begin{array}{lrrrrrrrr}\text { SD 0 } & 1,75 & 1,75 & 1,307 & 1,307 & 0 & 47,88 \% & 23,42 \% & 28,70 \% \\ \text { SD I } & 1,75 & 1,89 & 1,307 & 1,447 & -0,14 & & & \\ \text { "Chance" I } & 1,776 & 1,776 & 1,247 & 1,247 & 0 & 49,82 \% & 23,30 \% & 26,89 \% \\ \text { "Chance" II } & 3,315 & 1,717 & 2,923 & 1,324 & 1,598 & 46,65 \% & 23,62 \% & 29,64 \% \\ \text { Implícito I } & 1 & 1 & 0,9 & 0,9 & 0 & 36,79 \% & 31,77 \% & 31,44 \% \\ \text { Implícito II } & 1 & 1,22 & 0,9 & 1,12 & -0,22 & & & \end{array}$

Palmeiras 2x1 Atlético MG

$\begin{array}{lrrrrrrrr}\text { SD 0 } & 2,105 & 2,105 & 0,832 & 0,832 & 0 & 66,76 \% & 19,54 \% & 13,70 \% \\ \text { SD I } & 2,105 & 1,838 & 0,832 & 0,565 & 0,266 & 68,14 \% & 21,17 \% & 10,69 \% \\ \text { "Chance" I } & 2,256 & 2,256 & 0,864 & 0,864 & 0 & 68,85 \% & 18,24 \% & 12,91 \% \\ \text { "Chance" II } & 3,666 & 2,068 & 2,427 & 0,828 & 1,598 & 65,97 \% & 19,89 \% & 13,99 \% \\ \text { Implícito I } & 2 & 2 & 1 & 1 & 0 & 60,57 \% & 21,17 \% & 18,26 \% \\ \text { Implícito II } & 2 & 1,526 & 1 & 0,526 & 0,474 & 61,98 \% & 25,43 \% & 12,59 \%\end{array}$

São Paulo 6x1 América RN

$\begin{array}{lrrrrrrrr}\text { SD 0 } & 2,113 & 2,113 & 0,914 & 0,914 & 0 & 64,94 \% & 19,82 \% & 15,24 \% \\ \text { SD I } & 2,113 & 2,287 & 0,914 & 1,088 & -0,174 & & & \\ \text { "Chance" I } & 2,295 & 2,295 & 0,935 & 0,935 & 0 & 67,91 \% & 18,24 \% & 13,85 \% \\ \text { "Chance" II } & 3,715 & 2,117 & 2,486 & 0,888 & 1,598 & 65,49 \% & 19,67 \% & 14,67 \% \\ \text { Implícito I } & 1,1 & 1,1 & 0,9 & 0,9 & 0 & 39,91 \% & 30,64 \% & 29,45 \% \\ \text { Implícito II } & 1,1 & 1,14 & 0,9 & 0,94 & -0,04 & & & \end{array}$

Paraná 1x0 Ponte Preta

$\begin{array}{lrrrrrrrr}\text { SD 0 } & 1,165 & 1,165 & 0,666 & 0,666 & 0 & 47,83 \% & 31,09 \% & 21,08 \% \\ \text { SD I } & 1,165 & 0,714 & 0,666 & 0,215 & 0,451 & 44,02 \% & 45,77 \% & 10,21 \% \\ \text { "Chance" I } & 1,052 & 1,052 & 0,714 & 0,714 & 0 & 42,95 \% & 32,57 \% & 24,48 \% \\ \text { "Chance" II } & 2,673 & 1,074 & 2,199 & 0,601 & 1,598 & 46,68 \% & 32,91 \% & 20,39 \% \\ \text { Implícito I } & 1,4 & 1,4 & 1,3 & 1,3 & 0 & 39,41 \% & 25,78 \% & 34,81 \% \\ \text { Implícito II } & 1,4 & 1,003 & 1,3 & 0,903 & 0,397 & 36,81 \% & 31,72 \% & 31,47 \%\end{array}$

Implícito II $\quad 1,4 \quad 1,003 \quad 1,3 \quad 0,903 \quad 0,397 \quad 36,81 \% 31,72 \%$

Internacional 6x1 Goiás

$\begin{array}{lrrrrrrrr}\text { SD 0 } & 1,411 & 1,411 & 0,382 & 0,382 & 0 & 63,22 \% & 26,90 \% & 9,88 \% \\ \text { SD I } & 1,411 & 1,155 & 0,382 & 0,126 & 0,256 & 64,03 \% & 31,98 \% & 3,99 \% \\ \text { "Chance" I } & 1,423 & 1,423 & 0,579 & 0,579 & 0 & 57,76 \% & 27,14 \% & 15,09 \% \\ \text { "Chance" II } & 3,04 & 1,442 & 1,99 & 0,392 & 1,598 & 63,71 \% & 26,37 \% & 9,88 \% \\ \text { Implícito I } & 1,4 & 1,4 & 1 & 1 & 0 & 46,15 \% & 26,97 \% & 26,88 \% \\ \text { Implícito II } & 1,4 & 1,303 & 1 & 0,903 & 0,097 & 45,82 \% & 28,33 \% & 25,85 \%\end{array}$

Juventude 2x0 Vitória

$\begin{array}{lrrrrrrrr}\text { SD 0 } & 1,689 & 1,689 & 1,093 & 1,093 & 0 & 51,30 \% & 24,11 \% & 24,58 \% \\ \text { SD I } & 1,689 & 1,314 & 1,093 & 0,718 & 0,375 & 50,88 \% & 28,71 \% & 20,40 \% \\ \text { "Chance" I } & 1,618 & 1,618 & 1,014 & 1,014 & 0 & 51,44 \% & 24,82 \% & 23,74 \% \\ \text { "Chance" II } & 3,281 & 1,683 & 2,721 & 1,123 & 1,598 & 50,39 \% & 24,15 \% & 25,39 \% \\ \text { Implícito I } & 2 & 2 & 1,2 & 1,2 & 0 & 55,91 \% & 21,55 \% & 22,54 \% \\ \text { Implícito II } & 2 & 1,208 & 1,2 & 0,408 & 0,792 & 56,56 \% & 30,94 \% & 12,50 \%\end{array}$


Vasco 1x1 Flamengo

$\begin{array}{lrrrrrrrr}\text { SD 0 } & 1,941 & 1,941 & 1,128 & 1,128 & 0 & 56,28 \% & 21,95 \% & 21,77 \% \\ \text { SD I } & 1,941 & 1,81 & 1,128 & 0,997 & 0,131 & 56,45 \% & 22,96 \% & 20,59 \% \\ \text { "Chance" I } & 2,297 & 2,297 & 1,303 & 1,303 & 0 & 59,71 \% & 19,49 \% & 20,79 \% \\ \text { "Chance" II } & 3,64 & 2,127 & 2,854 & 1,342 & 1,513 & 55,30 \% & 20,77 \% & 23,78 \% \\ \text { Implícito I } & 1,9 & 1,9 & 1,2 & 1,2 & 0 & 53,72 \% & 22,35 \% & 23,94 \% \\ \text { Implícito II } & 1,9 & 1,103 & 1,2 & 0,403 & 0,797 & 53,35 \% & 33,20 \% & 13,45 \%\end{array}$

Grêmio 1x1 Palmeiras

$\begin{array}{lrrrrrrrr}\text { SD 0 } & 0,96 & 0,96 & 1,849 & 1,849 & 0 & 19,24 \% & 22,52 \% & 58,24 \% \\ \text { SD I } & 0,96 & 0,768 & 1,849 & 1,657 & 0,192 & 17,15 \% & 24,24 \% & 58,61 \% \\ \text { "Chance" I } & 0,884 & 0,884 & 1,727 & 1,727 & 0 & 19,05 \% & 23,64 \% & 57,31 \% \\ \text { "Chance" II } & 2,384 & 0,871 & 3,29 & 1,777 & 1,513 & 18,11 \% & 23,08 \% & 58,74 \% \\ \text { Implícito I } & 1 & 1 & 1,9 & 1,9 & 0 & 19,49 \% & 22,11 \% & 58,41 \% \\ \text { Implícito II } & 1 & 0,403 & 1,9 & 1,303 & 0,597 & 11,41 \% & 29,03 \% & 59,56 \%\end{array}$

Corinthians $3 \times 2$ Guarani

$\begin{array}{lrrrrrrrr}\text { SD 0 } & 1,638 & 1,638 & 0,871 & 0,871 & 0 & 55,49 \% & 24,60 \% & 19,91 \% \\ \text { SD I } & 1,638 & 1,738 & 0,871 & 0,971 & -0,1 & & & \\ \text { "Chance" I } & 1,666 & 1,666 & 0,913 & 0,913 & 0 & 55,10 \% & 24,33 \% & 20,57 \% \\ \text { "Chance" II } & 3,171 & 1,659 & 2,403 & 0,891 & 1,513 & 55,46 \% & 24,39 \% & 20,11 \% \\ \text { Implícito I } & 1,8 & 1,8 & 1 & 1 & 0 & 56,15 \% & 23,06 \% & 20,79 \% \\ \text { Implícito } & 1,8 & 1,408 & 1 & 0,608 & 0,392 & 56,54 \% & 27,41 \% & 16,06 \%\end{array}$

Implícito II $\quad 1,8 \quad 1,408 \quad 1 \quad 0,608=0,392 \quad 56,54 \% \quad 27,41 \%$

Coritiba 2x0 Goiás

$\begin{array}{lrrrrrrrr}\text { SD 0 } & 1,378 & 1,378 & 0,833 & 0,833 & 0 & 49,68 \% & 27,64 \% & 22,68 \% \\ \text { SD I } & 1,378 & 0,615 & 0,833 & 0,07 & 0,763 & 43,67 \% & 52,57 \% & 3,76 \% \\ \text { "Chance" I } & 1,318 & 1,318 & 0,827 & 0,827 & 0 & 48,15 \% & 28,40 \% & 23,45 \% \\ \text { "Chance" II } & 2,878 & 1,365 & 2,294 & 0,781 & 1,513 & 50,64 \% & 27,91 \% & 21,43 \% \\ \text { Implícito I } & 1,6 & 1,6 & 0,9 & 0,9 & 0 & 53,82 \% & 25,03 \% & 21,15 \% \\ \text { Implícito II } & 1,6 & 1,22 & 0,9 & 0,52 & 0,38 & 53,61 \% & 30,58 \% & 15,81 \%\end{array}$

Cruzeiro 0x2 Vitória

$\begin{array}{lrrrrrrrr}\text { SD 0 } & 1,835 & 1,835 & 1,165 & 1,165 & 0 & 53,05 \% & 22,86 \% & 24,09 \% \\ \text { SD I } & 1,835 & 1,728 & 1,165 & 1,058 & 0,108 & 53,07 \% & 23,77 \% & 23,16 \% \\ \text { "Chance" I } & 1,773 & 1,773 & 1,107 & 1,107 & 0 & 52,96 \% & 23,37 \% & 23,66 \% \\ \text { "Chance" II } & 3,3 & 1,787 & 2,681 & 1,168 & 1,513 & 51,82 \% & 23,25 \% & 24,86 \% \\ \text { Implícito I } & 2,1 & 2,1 & 1,4 & 1,4 & 0 & 53,61 \% & 21,00 \% & 25,39 \%\end{array}$

Sport 4x1 Paraná

$\begin{array}{lrrrrrrrr}\text { SD 0 } & 1,826 & 1,826 & 0,618 & 0,618 & 0 & 66,47 \% & 21,61 \% & 11,92 \% \\ \text { SD I } & 1,826 & 2,125 & 0,618 & 0,917 & -0,299 & & & \\ \text { "Chance" I } & 1,807 & 1,807 & 0,729 & 0,729 & 0 & 63,13 \% & 22,33 \% & 14,54 \% \\ \text { "Chance" II } & 3,266 & 1,753 & 2,116 & 0,603 & 1,513 & 65,19 \% & 22,48 \% & 12,27 \% \\ \text { Implícito I } & 2 & 2 & 1 & 1 & 0 & 60,57 \% & 21,17 \% & 18,26 \% \\ \text { Implícito II } & 2 & 1,303 & 1 & 0,303 & 0,697 & 62,67 \% & 28,82 \% & 8,51 \%\end{array}$


Bragantino 1x1 América RN

$\begin{array}{lrrrrrrrr}\text { SD 0 } & 1,708 & 1,708 & 1,331 & 1,331 & 0 & 46,36 \% & 23,67 \% & 29,97 \% \\ \text { SD I } & 1,708 & 1,881 & 1,331 & 1,504 & -0,173 & & & \\ \text { "Chance" I } & 1,6 & 1,6 & 1,241 & 1,241 & 0 & 45,73 \% & 24,62 \% & 29,65 \% \\ \text { "Chance" II } & 3,253 & 1,74 & 2,825 & 1,312 & 1,513 & 47,47 \% & 23,48 \% & 28,98 \% \\ \text { Implícito I } & 2,1 & 2,1 & 1,3 & 1,3 & 0 & 55,79 \% & 20,91 \% & 23,29 \% \\ \text { Implícito II } & 2,1 & 1,003 & 1,3 & 0,203 & 1,097 & 56,22 \% & 36,37 \% & 7,41 \%\end{array}$

América MG 0x0 Botafogo

$\begin{array}{lrrrrrrrr}\text { SD 0 } & 1,267 & 1,267 & 1,616 & 1,616 & 0 & 30,00 \% & 24,44 \% & 45,55 \% \\ \text { SD I } & 1,267 & 1,086 & 1,616 & 1,435 & 0,181 & 28,57 \% & 26,38 \% & 45,05 \% \\ \text { "Chance" I } & 1,177 & 1,177 & 1,507 & 1,507 & 0 & 29,68 \% & 25,50 \% & 44,83 \% \\ \text { "Chance" II } & 2,707 & 1,194 & 3,114 & 1,602 & 1,513 & 28,45 \% & 24,71 \% & 46,79 \% \\ \text { Implícito I } & 1 & 1 & 1,9 & 1,9 & 0 & 19,49 \% & 22,11 \% & 58,41 \% \\ \text { Implícito II } & 1 & 0,508 & 1,9 & 1,408 & 0,492 & 13,32 \% & 27,29 \% & 59,39 \%\end{array}$

Atlético PR 0x0 Juventude

$\begin{array}{lrrrrrrrr}\text { SD 0 } & 0,988 & 0,988 & 0,846 & 0,846 & 0 & 37,65 \% & 32,40 \% & 29,95 \% \\ \text { SD I } & 0,988 & 0,969 & 0,846 & 0,827 & 0,019 & 37,48 \% & 32,81 \% & 29,71 \% \\ \text { "Chance" I } & 0,915 & 0,915 & 0,886 & 0,886 & 0 & 34,35 \% & 32,86 \% & 32,78 \% \\ \text { "Chance" II } & 2,454 & 0,941 & 2,347 & 0,834 & 1,513 & 36,38 \% & 33,10 \% & 30,51 \% \\ \text { Implícito I } & 0,9 & 0,9 & 1,8 & 1,8 & 0 & 18,50 \% & 22,90 \% & 58,61 \% \\ \text { Implícito II } & 0,9 & 0,503 & 1,8 & 1,403 & 0,397 & 13,23 \% & 27,37 \% & 59,40 \%\end{array}$

Portuguesa 1x0 Atlético MG

$\begin{array}{lrrrrrrrr}\text { SD 0 } & 1,933 & 1,933 & 1,183 & 1,183 & 0 & 54,84 \% & 22,07 \% & 23,09 \% \\ \text { SD I } & 1,933 & 1,476 & 1,183 & 0,726 & 0,457 & 55,14 \% & 26,49 \% & 18,37 \% \\ \text { "Chance" I } & 1,987 & 1,987 & 1,118 & 1,118 & 0 & 57,52 \% & 21,54 \% & 20,94 \% \\ \text { "Chance" II } & 3,416 & 1,903 & 2,653 & 1,141 & 1,513 & 55,07 \% & 22,28 \% & 22,56 \% \\ \text { Implícito I } & 1,8 & 1,8 & 1,4 & 1,4 & 0 & 47,02 \% & 22,95 \% & 30,03 \% \\ \text { Implícito II } & 1,8 & 0,903 & 1,4 & 0,503 & 0,897 & 43,41 \% & 36,99 \% & 19,60 \%\end{array}$

Ponte Preta $1 \times 1$ São Paulo

$\begin{array}{lrrrrrrrr}\text { SD 0 } & 0,88 & 0,88 & 1,297 & 1,297 & 0 & 25,31 \% & 28,47 \% & 46,22 \% \\ \text { SD I } & 0,88 & 0,237 & 1,297 & 0,654 & 0,643 & 11,81 \% & 47,63 \% & 40,56 \% \\ \text { "Chance" I } & 0,794 & 0,794 & 1,261 & 1,261 & 0 & 23,43 \% & 29,22 \% & 47,35 \% \\ \text { "Chance" II } & 2,306 & 0,794 & 2,822 & 1,309 & 1,513 & 22,64 \% & 28,60 \% & 48,74 \% \\ \text { Implícito I } & 1 & 1 & 1,8 & 1,8 & 0 & 20,79 \% & 23,06 \% & 56,15 \% \\ \text { Implícito II } & 1 & 0,608 & 1,8 & 1,408 & 0,392 & 16,06 \% & 27,41 \% & 56,54 \%\end{array}$

Santos 2x0 Internacional

$\begin{array}{lrrrrrrrr}\text { SD 0 } & 1,612 & 1,612 & 1,035 & 1,035 & 0 & 50,80 \% & 24,86 \% & 24,35 \% \\ \text { SD I } & 1,612 & 1,279 & 1,035 & 0,702 & 0,333 & 50,30 \% & 29,25 \% & 20,45 \% \\ \text { "Chance" I } & 1,447 & 1,447 & 1,119 & 1,119 & 0 & 44,60 \% & 26,17 \% & 29,23 \% \\ \text { "Chance" II } & 3,079 & 1,566 & 2,658 & 1,145 & 1,513 & 47,02 \% & 25,10 \% & 27,83 \% \\ \text { Implícito I } & 1,6 & 1,6 & 1,4 & 1,4 & 0 & 42,31 \% & 24,17 \% & 33,52 \% \\ \text { Implícito II } & 1,6 & 0,807 & 1,4 & 0,607 & 0,793 & 37,10 \% & 37,76 \% & 25,14 \%\end{array}$


América MG 2x1 Ponte Preta

$\begin{array}{lrrrrrrrr}\text { SD 0 } & 1,254 & 1,254 & 0,776 & 0,776 & 0 & 47,61 \% & 29,38 \% & 23,01 \% \\ \text { SD I } & 1,254 & 0,677 & 0,776 & 0,199 & 0,577 & 42,77 \% & 47,45 \% & 9,78 \% \\ \text { "Chance" I } & 1,161 & 1,161 & 0,754 & 0,754 & 0 & 45,40 \% & 30,74 \% & 23,86 \% \\ \text { "Chance" II } & 2,585 & 1,187 & 2,172 & 0,774 & 1,398 & 45,67 \% & 30,28 \% & 24,04 \% \\ \text { Implícito I } & 1,8 & 1,8 & 1 & 1 & 0 & 56,15 \% & 23,06 \% & 20,79 \% \\ \text { Implícito II } & 1,8 & 1,408 & 1 & 0,608 & 0,392 & 56,54 \% & 27,41 \% & 16,06 \%\end{array}$

Guarani $1 \times 1$ Santos

$\begin{array}{lrrrrrrrr}\text { SD 0 } & 1,543 & 1,543 & 1,486 & 1,486 & 0 & 39,17 \% & 24,16 \% & 36,67 \% \\ \text { SD I } & 1,543 & 1,628 & 1,486 & 1,571 & -0,085 & & \\ \text { "Chance" I } & 1,527 & 1,527 & 1,508 & 1,508 & 0 & 38,35 \% & 24,14 \% & 37,51 \% \\ \text { "Chance" II } & 2,947 & 1,549 & 2,98 & 1,582 & 1,398 & 37,39 \% & 23,71 \% & 38,84 \% \\ \text { Implícito I } & 1 & 1 & 1,8 & 1,8 & 0 & 20,79 \% & 23,06 \% & 56,15 \% \\ \text { Implícito II } & 1 & 0,726 & 1,8 & 1,526 & 0,274 & 17,72 \% & 25,83 \% & 56,45 \%\end{array}$

Internacional 2x1 Bragantino

$\begin{array}{lrrrrrrrr}\text { SD 0 } & 2,022 & 2,022 & 0,405 & 0,405 & 0 & 75,99 \% & 17,68 \% & 6,33 \% \\ \text { SD I } & 2,022 & 1,818 & 0,405 & 0,201 & 0,204 & 77,90 \% & 18,59 \% & 3,51 \% \\ \text { "Chance" I } & 2,055 & 2,055 & 0,606 & 0,606 & 0 & 71,39 \% & 18,81 \% & 9,80 \% \\ \text { "Chance" II } & 3,379 & 1,981 & 1,83 & 0,432 & 1,398 & 74,47 \% & 18,42 \% & 7,04 \% \\ \text { Implícito I } & 2,1 & 2,1 & 0,8 & 0,8 & 0 & 67,44 \% & 19,43 \% & 13,13 \% \\ \text { Implícito II } & 2,1 & 1,608 & 0,8 & 0,308 & 0,492 & 70,37 \% & 22,96 \% & 6,66 \%\end{array}$

Vitória 1x0 Paraná

$\begin{array}{lrrrrrrrr}\text { SD 0 } & 2,143 & 2,143 & 1,1 & 1,1 & 0 & 61,18 \% & 20,18 \% & 18,63 \% \\ \text { SD I } & 2,143 & 2,448 & 1,1 & 1,406 & -0,306 & & \\ \text { "Chance" I } & 2,176 & 2,176 & 1,02 & 1,02 & 0 & 63,69 \% & 19,65 \% & 16,66 \% \\ \text { "Chance" II } & 3,446 & 2,048 & 2,486 & 1,088 & 1,398 & 59,41 \% & 20,96 \% & 19,53 \% \\ \text { Implícito I } & 1,9 & 1,9 & 0,9 & 0,9 & 0 & 60,84 \% & 21,87 \% & 17,29 \% \\ \text { Implícito II } & 1,9 & 1,403 & 0,9 & 0,403 & 0,497 & 62,37 \% & 27,13 \% & 10,50 \%\end{array}$

Implícito II $\quad 1,9 \quad 1,403 \quad 0,9 \quad 0,403 \quad 0,497 \quad 62,37 \% \quad 27,13 \% \quad 10,50 \%$

Vasco 1x2 Portuguesa

\begin{tabular}{|c|c|c|c|c|c|c|c|c|}
\hline SD 0 & 1,764 & 1,764 & 0,909 & 0,909 & 0 & $57,55 \%$ & $23,29 \%$ & $19,16 \%$ \\
\hline SD I & 1,764 & 1,262 & 0,909 & 0,407 & 0,503 & $58,23 \%$ & $29,85 \%$ & $11,92 \%$ \\
\hline "Chance" I & 1,705 & 1,705 & 0,939 & 0,939 & 0 & $55,42 \%$ & $23,94 \%$ & $20,64 \%$ \\
\hline "Chance" II & 3,095 & 1,697 & 2,331 & 0,933 & 1,398 & $55,33 \%$ & $24,01 \%$ & $20,61 \%$ \\
\hline Implícito I & 1,9 & 1,9 & 1,1 & 1,1 & 0 & $56,03 \%$ & $22,26 \%$ & $21,71 \%$ \\
\hline Implícito II & 1,9 & 1,308 & 1,1 & 0,508 & 0,592 & $56,58 \%$ & $29,01 \%$ & $14,41 \%$ \\
\hline Palmeiras $2 \times 1$ & Flamengo & & & & & & & \\
\hline SD 0 & 2,109 & 2,109 & 0,664 & 0,664 & 0 & $70,96 \%$ & $18,58 \%$ & $10,46 \%$ \\
\hline SD I & 2,109 & 2,188 & 0,664 & 0,742 & $-0,078$ & & & \\
\hline "Chance" I & 2,243 & 2,243 & 0,802 & 0,802 & 0 & $70,07 \%$ & $18,03 \%$ & $11,90 \%$ \\
\hline "Chance" II & 3,434 & 2,086 & 2,069 & 0,72 & 1,349 & $69,04 \%$ & $19,16 \%$ & $11,71 \%$ \\
\hline Implícito I & 1,9 & 1,9 & 1,2 & 1,2 & 0 & $53,72 \%$ & $22,35 \%$ & $23,94 \%$ \\
\hline Implícito II & 1,9 & 1,103 & 1,2 & 0,403 & 0,797 & $53,35 \%$ & $33,20 \%$ & $13,45 \%$ \\
\hline
\end{tabular}


Bragantino 1xo Corinthians

$\begin{array}{lrrrrrrrr}\text { SD 0 } & 1,056 & 1,056 & 1,463 & 1,463 & 0 & 27,30 \% & 26,21 \% & 46,49 \% \\ \text { SD I } & 1,056 & 1,533 & 1,463 & 1,941 & -0,477 & & & \\ \text { "Chance" I } & 0,981 & 0,981 & 1,391 & 1,391 & 0 & 26,52 \% & 27,12 \% & 46,36 \% \\ \text { "Chance" II } & 2,368 & 1,02 & 2,815 & 1,466 & 1,349 & 26,28 \% & 26,27 \% & 47,43 \% \\ \text { Implícito I } & 0,9 & 0,9 & 2 & 2 & 0 & 16,16 \% & 20,86 \% & 62,98 \% \\ \text { Implícito II } & 0,9 & 0,408 & 2 & 1,508 & 0,492 & 9,76 \% & 25,27 \% & 64,97 \%\end{array}$

Grêmio 2x1 Vitória

$\begin{array}{lrrrrrrrr}\text { SD 0 } & 1,695 & 1,695 & 1,848 & 1,848 & 0 & 35,85 \% & 22,07 \% & 42,08 \% \\ \text { SD I } & 1,695 & 1,819 & 1,848 & 1,972 & -0,123 & & & \\ \text { "Chance" I } & 1,577 & 1,577 & 1,72 & 1,72 & 0 & 35,50 \% & 22,98 \% & 41,52 \% \\ \text { "Chance" II } & 2,974 & 1,625 & 3,141 & 1,792 & 1,349 & 35,27 \% & 22,50 \% & 42,16 \% \\ \text { Implícito I } & 1,5 & 1,5 & 1,5 & 1,5 & 0 & 37,85 \% & 24,30 \% & 37,85 \% \\ \text { Implícito II } & 1,5 & 0,707 & 1,5 & 0,707 & 0,793 & 30,97 \% & 38,07 \% & 30,97 \%\end{array}$

Vasco 2 xo Cruzeiro

$\begin{array}{lrrrrrrrr}\text { SD 0 } & 1,612 & 1,612 & 0,635 & 0,635 & 0 & 61,08 \% & 24,50 \% & 14,42 \% \\ \text { SD I } & 1,612 & 1,068 & 0,635 & 0,091 & 0,544 & 62,36 \% & 34,52 \% & 3,12 \% \\ \text { "Chance" I } & 1,549 & 1,549 & 0,744 & 0,744 & 0 & 56,56 \% & 25,55 \% & 17,89 \% \\ \text { "Chance" II } & 2,917 & 1,568 & 1,976 & 0,627 & 1,349 & 60,17 \% & 25,09 \% & 14,72 \% \\ \text { Implícito I } & 1,7 & 1,7 & 1,3 & 1,3 & 0 & 46,85 \% & 23,78 \% & 29,37 \% \\ \text { Implícito II } & 1,7 & 1,108 & 1,3 & 0,708 & 0,592 & 44,92 \% & 31,76 \% & 23,31 \%\end{array}$

Implícito II $\quad 1,7 \quad 1,108 \quad 1,3 \quad 0,708 \quad 0,592 \quad 44,92 \% \quad 31,76 \%$

Portuguesa $5 \times 2$ Botafogo

$\begin{array}{lrrrrrrrr}\text { SD 0 } & 1,512 & 1,512 & 1,07 & 1,07 & 0 & 47,44 \% & 25,73 \% & 26,83 \% \\ \text { SD I } & 1,512 & 1,135 & 1,07 & 0,693 & 0,377 & 46,17 \% & 31,44 \% & 22,39 \% \\ \text { "Chance" I } & 1,461 & 1,461 & 1,042 & 1,042 & 0 & 46,77 \% & 26,26 \% & 26,96 \% \\ \text { "Chance" II } & 2,829 & 1,48 & 2,388 & 1,039 & 1,349 & 47,32 \% & 26,09 \% & 26,56 \% \\ \text { Implícito I } & 1,5 & 1,5 & 1,4 & 1,4 & 0 & 39,86 \% & 24,74 \% & 35,40 \% \\ \text { Implícito II } & 1,5 & 0,903 & 1,4 & 0,803 & 0,597 & 35,83 \% & 33,92 \% & 30,25 \%\end{array}$

Atlético MG $1 \times 0$ São Paulo

\begin{tabular}{|c|c|c|c|c|c|c|c|c|}
\hline SD 0 & 1,6 & 1,6 & 1,624 & 1,624 & 0 & $37,83 \%$ & $23,33 \%$ & $38,85 \%$ \\
\hline SD I & 1,6 & 1,098 & 1,624 & 1,122 & 0,502 & $34,91 \%$ & $28,97 \%$ & $36,12 \%$ \\
\hline "Chance" I & 1,512 & 1,512 & 1,574 & 1,574 & 0 & $36,70 \%$ & $23,90 \%$ & $39,40 \%$ \\
\hline "Chance" II & 2,879 & 1,53 & 2,967 & 1,618 & 1,349 & $36,28 \%$ & $23,62 \%$ & $40,05 \%$ \\
\hline Implícito I & 1 & 1 & 1,1 & 1,1 & 0 & $32,48 \%$ & $29,91 \%$ & $37,61 \%$ \\
\hline Implícito II & 1 & 1,308 & 1,1 & 1,408 & $-0,308$ & & & \\
\hline
\end{tabular}

Santos 0x0 sport

$\begin{array}{lrrrrrrrr}\text { SD 0 } & 1,834 & 1,834 & 1,143 & 1,143 & 0 & 53,54 \% & 22,86 \% & 23,60 \% \\ \text { SD I } & 1,834 & 2,33 & 1,143 & 1,639 & -0,496 & & \\ \text { "Chance" I } & 1,87 & 1,87 & 1,174 & 1,174 & 0 & 53,63 \% & 22,58 \% & 23,79 \% \\ \text { "Chance" II } & 3,181 & 1,833 & 2,57 & 1,222 & 1,349 & 51,65 \% & 22,88 \% & 25,41 \% \\ \text { Implícito I } & 1,8 & 1,8 & 1,7 & 1,7 & 0 & 40,92 \% & 22,25 \% & 36,82 \% \\ \text { Implícito II } & 1,8 & 0,826 & 1,7 & 0,726 & 0,974 & 34,93 \% & 35,93 \% & 29,14 \%\end{array}$


Internacional $1 \times 3$ Guarani

$\begin{array}{lrrrrrrrr}\text { SD 0 } & 1,768 & 1,768 & 0,855 & 0,855 & 0 & 59,00 \% & 23,14 \% & 17,86 \% \\ \text { SD I } & 1,768 & 1,238 & 0,855 & 0,325 & 0,53 & 60,05 \% & 30,27 \% & 9,68 \% \\ \text { "Chance" I } & 1,819 & 1,819 & 0,879 & 0,879 & 0 & 59,55 \% & 22,65 \% & 17,79 \% \\ \text { "Chance" II } & 3,139 & 1,79 & 2,231 & 0,882 & 1,349 & 58,78 \% & 22,96 \% & 18,21 \% \\ \text { Implícito I } & 1,9 & 1,9 & 1,6 & 1,6 & 0 & 45,11 \% & 22,04 \% & 32,85 \% \\ \text { Implícito II } & 1,9 & 0,808 & 1,6 & 0,508 & 1,092 & 39,67 \% & 39,01 \% & 21,32 \%\end{array}$

Ponte Preta $1 \times 0$ Juventude

$\begin{array}{lrrrrrrrr}\text { SD 0 } & 0,516 & 0,516 & 0,696 & 0,696 & 0 & 23,59 \% & 41,45 \% & 34,96 \% \\ \text { SD I } & 0,516 & 0,624 & 0,696 & 0,804 & -0,108 & & & \\ \text { "Chance" I } & 0,601 & 0,601 & 0,815 & 0,815 & 0 & 24,75 \% & 37,70 \% & 37,55 \% \\ \text { "Chance" II } & 1,843 & 0,494 & 2,078 & 0,729 & 1,349 & 22,10 \% & 41,02 \% & 36,87 \% \\ \text { Implícito I } & 1 & 1 & 1,7 & 1,7 & 0 & 22,18 \% & 24,02 \% & 53,79 \% \\ \text { Implícito II } & 1 & 0,603 & 1,7 & 1,303 & 0,397 & 17,22 \% & 29,06 \% & 53,71 \%\end{array}$

Paraná 1xo Atlético PR

$\begin{array}{lrrrrrrrr}\text { SD 0 } & 1,058 & 1,058 & 1,368 & 1,368 & 0 & 29,01 \% & 27,08 \% & 43,91 \% \\ \text { SD I } & 1,058 & 1,323 & 1,368 & 1,633 & -0,265 & & & \\ \text { "Chance" I } & 1,087 & 1,087 & 1,418 & 1,418 & 0 & 28,91 \% & 26,53 \% & 44,57 \% \\ \text { "Chance" II } & 2,475 & 1,126 & 2,787 & 1,439 & 1,349 & 29,56 \% & 26,21 \% & 44,20 \% \\ \text { Implícito I } & 1,8 & 1,8 & 1,5 & 1,5 & 0 & 44,92 \% & 22,77 \% & 32,32 \% \\ \text { Implícito II } & 1,8 & 0,707 & 1,5 & 0,407 & 1,093 & 38,23 \% & 42,96 \% & 18,81 \%\end{array}$

Implícito II $\quad 1,8 \quad 0,707 \quad 1,5 \quad 0,407 \quad 1,093 \quad 38,23 \% \quad 42,96 \%$

América RN 0x1 Coritiba

$\begin{array}{lrrrrrrrr}\text { SD 0 } & 1,556 & 1,556 & 1,219 & 1,219 & 0 & 45,14 \% & 25,00 \% & 29,86 \% \\ \text { SD I } & 1,556 & 1,214 & 1,219 & 0,877 & 0,342 & 43,89 \% & 29,46 \% & 26,65 \% \\ \text { "Chance" I } & 1,452 & 1,452 & 1,152 & 1,152 & 0 & 43,98 \% & 26,02 \% & 30,00 \% \\ \text { "Chance" II } & 2,797 & 1,449 & 2,611 & 1,262 & 1,349 & 41,45 \% & 25,63 \% & 32,90 \% \\ \text { Implícito I } & 1,2 & 1,2 & 1,3 & 1,3 & 0 & 34,14 \% & 26,96 \% & 38,90 \% \\ \text { Implícito II } & 1,2 & 0,907 & 1,3 & 1,007 & 0,293 & 31,52 \% & 31,62 \% & 36,85 \%\end{array}$

Implícito II $\quad 1,2 \quad 0,907 \quad 1,3 \quad 1,007 \quad 0,293 \quad 31,52 \% \quad 31,62 \% \quad 36,85 \%$

América MG 1x0 Goiás

$\begin{array}{lrrrrrrrr}\text { SD 0 } & 1,526 & 1,526 & 1,056 & 1,056 & 0 & 48,13 \% & 25,63 \% & 26,24 \% \\ \text { SD I } & 1,526 & 0,946 & 1,056 & 0,476 & 0,581 & 45,74 \% & 36,29 \% & 17,97 \% \\ \text { "Chance" I } & 1,49 & 1,49 & 0,942 & 0,942 & 0 & 49,96 \% & 26,19 \% & 23,86 \% \\ \text { "Chance" II } & 2,873 & 1,524 & 2,388 & 1,039 & 1,349 & 48,45 \% & 25,68 \% & 25,84 \% \\ \text { Implícito I } & 1,3 & 1,3 & 0,9 & 0,9 & 0 & 45,81 \% & 28,37 \% & 25,82 \% \\ \text { Implícito II } & 1,3 & 1,307 & 0,9 & 0,907 & -0,007 & & & \end{array}$

Cruzeiro 1x1 Flamengo

$\begin{array}{lrrrrrrrr}\text { SD 0 } & 1,632 & 1,632 & 0,903 & 0,903 & 0 & 54,54 \% & 24,68 \% & 20,78 \% \\ \text { SD I } & 1,632 & 1,257 & 0,903 & 0,528 & 0,376 & 54,49 \% & 29,92 \% & 15,59 \% \\ \text { "Chance" I } & 1,513 & 1,513 & 0,965 & 0,965 & 0 & 49,99 \% & 25,91 \% & 24,10 \% \\ \text { "Chance" II } & 2,971 & 1,581 & 2,33 & 0,939 & 1,39 & 52,33 \% & 25,23 \% & 22,41 \% \\ \text { Implícito I } & 2 & 2 & 1 & 1 & 0 & 60,57 \% & 21,17 \% & 18,26 \% \\ \text { Implícito II } & 2 & 1,303 & 1 & 0,303 & 0,697 & 62,67 \% & 28,82 \% & 8,51 \%\end{array}$


Guarani 3×2 Palmeiras

$\begin{array}{lrrrrrrrr}\text { SD 0 } & 1,364 & 1,364 & 1,383 & 1,383 & 0 & 36,79 \% & 25,56 \% & 37,65 \% \\ \text { SD I } & 1,364 & 1,665 & 1,383 & 1,684 & -0,301 & & & \\ \text { "Chance" I } & 1,264 & 1,264 & 1,39 & 1,39 & 0 & 34,07 \% & 26,01 \% & 39,91 \% \\ \text { "Chance" II } & 2,722 & 1,331 & 2,84 & 1,45 & 1,39 & 34,63 \% & 25,33 \% & 40,01 \% \\ \text { Implícito I } & 1,2 & 1,2 & 2,1 & 2,1 & 0 & 21,21 \% & 20,76 \% & 58,03 \% \\ \text { Implícito II } & 1,2 & 0,203 & 2,1 & 1,103 & 0,997 & 6,77 \% & 33,52 \% & 59,71 \%\end{array}$

Vasco 0x0 sport

$\begin{array}{lrrrrrrrr}\text { SD 0 } & 1,674 & 1,674 & 1,009 & 1,009 & 0 & 52,95 \% & 24,28 \% & 22,78 \% \\ \text { SD I } & 1,674 & 1,535 & 1,009 & 0,87 & 0,139 & 52,92 \% & 25,76 \% & 21,32 \% \\ \text { "Chance" I } & 1,651 & 1,651 & 1,022 & 1,022 & 0 & 52,06 \% & 24,50 \% & 23,44 \% \\ \text { "Chance" II } & 3,048 & 1,658 & 2,445 & 1,054 & 1,39 & 51,43 \% & 24,41 \% & 24,12 \% \\ \text { Implícito I } & 1,8 & 1,8 & 1,7 & 1,7 & 0 & 40,92 \% & 22,25 \% & 36,82 \% \\ \text { Implícito II } & 1,8 & 0,603 & 1,7 & 0,503 & 1,197 & 31,30 \% & 43,93 \% & 24,77 \%\end{array}$

Implícito II

$1,8 \quad 0,603 \quad 1,7 \quad 0,503$

$1,197 \quad 31,30 \% \quad 43,93 \% \quad 24,77 \%$

Corinthians 3x0 Portuguesa

$\begin{array}{lrrrrrrrr}\text { SD 0 } & 1,379 & 1,379 & 1,438 & 1,438 & 0 & 36,08 \% & 25,18 \% & 38,74 \% \\ \text { SD I } & 1,379 & 1,775 & 1,438 & 1,834 & -0,396 & & & \\ \text { "Chance" I } & 1,493 & 1,493 & 1,566 & 1,566 & 0 & 36,39 \% & 24,01 \% & 39,60 \% \\ \text { "Chance" II } & 2,889 & 1,499 & 2,995 & 1,605 & 1,39 & 35,79 \% & 23,80 \% & 40,36 \% \\ \text { Implícito I } & 1,9 & 1,9 & 1,6 & 1,6 & 0 & 45,11 \% & 22,04 \% & 32,85 \% \\ \text { Implícito II } & 1,9 & 0,808 & 1,6 & 0,508 & 1,092 & 39,67 \% & 39,01 \% & 21,32 \%\end{array}$

Bragantino 1x2 São Paulo

$\begin{array}{lrrrrrrrr}\text { SD 0 } & 1,156 & 1,156 & 1,632 & 1,632 & 0 & 27,02 \% & 24,54 \% & 48,45 \% \\ \text { SD I } & 1,156 & 1,246 & 1,632 & 1,721 & -0,09 & & & \\ \text { "Chance" I } & 1,06 & 1,06 & 1,551 & 1,551 & 0 & 25,93 \% & 25,40 \% & 48,67 \% \\ \text { "Chance" II } & 2,495 & 1,105 & 3,005 & 1,615 & 1,39 & 26,02 \% & 24,75 \% & 49,19 \% \\ \text { Implícito I } & 1,4 & 1,4 & 1,4 & 1,4 & 0 & 37,36 \% & 25,28 \% & 37,36 \% \\ \text { Implícito II } & 1,4 & 0,807 & 1,4 & 0,807 & 0,593 & 32,43 \% & 35,14 \% & 32,43 \%\end{array}$

Paraná 1x2 Grêmio

$\begin{array}{lrrrrrrrr}\text { SD 0 } & 1,671 & 1,671 & 0,992 & 0,992 & 0 & 53,29 \% & 24,30 \% & 22,41 \% \\ \text { SD I } & 1,671 & 1,226 & 0,992 & 0,547 & 0,445 & 53,01 \% & 30,44 \% & 16,55 \% \\ \text { "Chance" I } & 1,414 & 1,414 & 0,924 & 0,924 & 0 & 48,38 \% & 27,03 \% & 24,60 \% \\ \text { "Chance" II } & 2,91 & 1,519 & 2,288 & 0,898 & 1,39 & 51,80 \% & 25,92 \% & 22,26 \% \\ \text { Implícito I } & 1,8 & 1,8 & 1,6 & 1,6 & 0 & 42,88 \% & 22,53 \% & 34,58 \%\end{array}$

Implícito II $\quad 1,8 \quad 0,703 \quad 1,6 \quad 0,503 \quad 1,097 \quad 35,57 \% \quad 41,52 \%$

Vitória $0 \times 3$ Coritiba

$\begin{array}{lrrrrrrrr}\text { SD 0 } & 1,89 & 1,89 & 1,126 & 1,126 & 0 & 55,20 \% & 22,38 \% & 22,42 \% \\ \text { SD I } & 1,89 & 1,943 & 1,126 & 1,18 & -0,053 & & & \\ \text { "Chance" I } & 1,863 & 1,863 & 1,082 & 1,082 & 0 & 55,63 \% & 22,57 \% & 21,80 \% \\ \text { "Chance" II } & 3,211 & 1,82 & 2,513 & 1,123 & 1,39 & 53,64 \% & 22,97 \% & 23,34 \% \\ \text { Implícito I } & 2 & 2 & 1 & 1 & 0 & 60,57 \% & 21,17 \% & 18,26 \% \\ \text { Implícito II } & 2 & 1,408 & 1 & 0,408 & 0,592 & 62,35 \% & 27,04 \% & 10,60 \%\end{array}$


América RN 2x2 Juventude

$\begin{array}{lrrrrrrrr}\text { SD 0 } & 0,996 & 0,996 & 1,14 & 1,14 & 0 & 31,56 \% & 29,55 \% & 38,89 \% \\ \text { SD I } & 0,996 & 0,5 & 1,14 & 0,644 & 0,496 & 23,88 \% & 42,97 \% & 33,16 \% \\ \text { "Chance" I } & 0,913 & 0,913 & 1,101 & 1,101 & 0 & 29,83 \% & 30,53 \% & 39,64 \% \\ \text { "Chance" II } & 2,341 & 0,951 & 2,628 & 1,237 & 1,39 & 28,39 \% & 28,83 \% & 42,77 \% \\ \text { Implícito I } & 0,9 & 0,9 & 1,3 & 1,3 & 0 & 25,82 \% & 28,37 \% & 45,81 \% \\ \text { Implícito II } & 0,9 & 0,907 & 1,3 & 1,307 & -0,007 & & & \end{array}$

Goiás 2x3 Atlético PR

$\begin{array}{lrrrrrrrr}\text { SD 0 } & 1,209 & 1,209 & 0,959 & 0,959 & 0 & 41,77 \% & 29,08 \% & 29,15 \% \\ \text { SD I } & 1,209 & 0,677 & 0,959 & 0,427 & 0,532 & 36,41 \% & 43,43 \% & 20,16 \% \\ \text { "Chance" I } & 1,043 & 1,043 & 1 & 1 & 0 & 35,88 \% & 30,44 \% & 33,68 \% \\ \text { "Chance" II } & 2,503 & 1,112 & 2,394 & 1,003 & 1,39 & 37,90 \% & 29,77 \% & 32,33 \% \\ \text { Implícito I } & 1,4 & 1,4 & 1,3 & 1,3 & 0 & 39,41 \% & 25,78 \% & 34,81 \% \\ \text { Implícito II } & 1,4 & 0,907 & 1,3 & 0,807 & 0,493 & 35,88 \% & 33,81 \% & 30,31 \%\end{array}$

Implícito II $\quad 1,4 \quad 0,907 \quad 1,3 \quad 0,807 \quad 0,493 \quad 35,88 \% \quad 33,81 \% \quad 30,31 \%$

São Paulo 1x1 Vasco

$\begin{array}{lrrrrrrrr}\text { SD 0 } & 1,614 & 1,614 & 1,139 & 1,139 & 0 & 48,40 \% & 24,71 \% & 26,89 \% \\ \text { SD I } & 1,614 & 1,09 & 1,139 & 0,615 & 0,524 & 46,82 \% & 32,56 \% & 20,61 \% \\ \text { "Chance" I } & 1,5 & 1,5 & 1,123 & 1,123 & 0 & 45,89 \% & 25,71 \% & 28,40 \% \\ \text { "Chance" II } & 0,875 & 1,557 & 0,464 & 1,146 & -0,682 & & & \\ \text { Implícito I } & 1 & 1 & 1,8 & 1,8 & 0 & 20,79 \% & 23,06 \% & 56,15 \% \\ \text { Implícito II } & 1 & 0,503 & 1,8 & 1,303 & 0,497 & 14,31 \% & 29,11 \% & 56,58 \%\end{array}$

Sport 0x2 Corinthians

$\begin{array}{lrrrrrrrr}\text { SD 0 } & 1,467 & 1,467 & 0,931 & 0,931 & 0 & 49,62 \% & 26,45 \% & 23,94 \% \\ \text { SD I } & 1,467 & 1,661 & 0,931 & 1,125 & -0,194 & & & \\ \text { "Chance" I } & 1,4 & 1,4 & 0,962 & 0,962 & 0 & 47,08 \% & 27,08 \% & 25,84 \% \\ \text { "Chance" II } & 0,763 & 1,445 & 0,266 & 0,948 & -0,682 & & & \\ \text { Implícito I } & 1,4 & 1,4 & 1,4 & 1,4 & 0 & 37,36 \% & 25,28 \% & 37,36 \% \\ \text { Implícito II } & 1,4 & 0,903 & 1,4 & 0,903 & 0,497 & 33,59 \% & 32,83 \% & 33,59 \%\end{array}$

Grêmio 3x2 Santos

$\begin{array}{lrrrrrrrr}\text { SD 0 } & 1,232 & 1,232 & 1,731 & 1,731 & 0 & 27,25 \% & 23,65 \% & 49,10 \% \\ \text { SD I } & 1,232 & 1,062 & 1,731 & 1,561 & 0,17 & 25,83 \% & 25,30 \% & 48,87 \% \\ \text { "Chance" I } & 1,156 & 1,156 & 1,659 & 1,659 & 0 & 26,57 \% & 24,31 \% & 49,11 \% \\ \text { "Chance" II } & 0,499 & 1,181 & 1,022 & 1,704 & -0,682 & & & \\ \text { Implícito I } & 1,1 & 1,1 & 2 & 2 & 0 & 20,39 \% & 21,40 \% & 58,21 \% \\ \text { Implícito II } & 1,1 & 0,303 & 2 & 1,203 & 0,797 & 9,28 \% & 31,04 \% & 59,68 \%\end{array}$

Flamengo $2 \times 3$ Portuguesa

$\begin{array}{lrrrrrrrr}\text { SD 0 } & 1,598 & 1,598 & 1,534 & 1,534 & 0 & 39,53 \% & 23,70 \% & 36,77 \% \\ \text { SD I } & 1,598 & 1,42 & 1,534 & 1,356 & 0,178 & 38,76 \% & 25,39 \% & 35,85 \% \\ \text { "Chance" I } & 1,571 & 1,571 & 1,493 & 1,493 & 0 & 39,71 \% & 23,99 \% & 36,31 \% \\ \text { "Chance" II } & 0,885 & 1,567 & 0,88 & 1,562 & -0,682 & & & \\ \text { Implícito I } & 1 & 1 & 1,9 & 1,9 & 0 & 19,49 \% & 22,11 \% & 58,41 \% \\ \text { Implícito II } & 1 & 0,508 & 1,9 & 1,408 & 0,492 & 13,32 \% & 27,29 \% & 59,39 \%\end{array}$


Botafogo 3×3 Paraná

$\begin{array}{lrrrrrrrr}\text { SD 0 } & 1,855 & 1,855 & 0,596 & 0,596 & 0 & 67,67 \% & 21,13 \% & 11,19 \% \\ \text { SD I } & 1,855 & 1,304 & 0,596 & 0,045 & 0,551 & 71,27 \% & 27,49 \% & 1,24 \% \\ \text { "Chance" I } & 1,783 & 1,783 & 0,71 & 0,71 & 0 & 63,08 \% & 22,55 \% & 14,37 \% \\ \text { "Chance" II } & 1,05 & 1,732 & -0,103 & 0,579 & -0,682 & & & \\ \text { Implícito I } & 1,3 & 1,3 & 1,3 & 1,3 & 0 & 36,80 \% & 26,39 \% & 36,80 \% \\ \text { Implícito II } & 1,3 & 1,003 & 1,3 & 1,003 & 0,297 & 34,60 \% & 30,80 \% & 34,60 \%\end{array}$

Palmeiras $3 \times 0$ Ponte Preta

$\begin{array}{lrrrrrrrr}\text { SD 0 } & 1,752 & 1,752 & 0,168 & 0,168 & 0 & 77,61 \% & 19,30 \% & 3,08 \% \\ \text { SD I } & 1,752 & 1,889 & 0,168 & 0,305 & -0,137 & & & \\ \text { "Chance" I } & 1,7 & 1,7 & 0,502 & 0,502 & 0 & 66,86 \% & 22,71 \% & 10,44 \% \\ \text { "Chance" II } & 1,013 & 1,695 & -0,495 & 0,187 & -0,682 & & & \\ \text { Implícito I } & 2 & 2 & 0,7 & 0,7 & 0 & 67,95 \% & 19,98 \% & 12,07 \% \\ \text { Implícito II } & 2 & 1,708 & 0,7 & 0,408 & 0,292 & 69,69 \% & 22,03 \% & 8,28 \%\end{array}$

Guarani 2x2 Cruzeiro

$\begin{array}{lrrrrrrrr}\text { SD 0 } & 1,626 & 1,626 & 0,91 & 0,91 & 0 & 54,21 \% & 24,75 \% & 21,04 \% \\ \text { SD I } & 1,626 & 1,37 & 0,91 & 0,654 & 0,256 & 54,21 \% & 27,99 \% & 17,80 \% \\ \text { "Chance" I } & 1,553 & 1,553 & 0,945 & 0,945 & 0 & 51,50 \% & 25,52 \% & 22,98 \% \\ \text { "Chance" II } & 0,895 & 1,577 & 0,261 & 0,943 & -0,682 & & & \\ \text { Implícito I } & 2 & 2 & 1,3 & 1,3 & 0 & 53,67 \% & 21,64 \% & 24,70 \% \\ \text { Implícito II } & 2 & 1,108 & 1,3 & 0,408 & 0,892 & 53,36 \% & 33,07 \% & 13,57 \%\end{array}$

Atlético MG 3x2 Atlético PR

$\begin{array}{lrrrrrrrr}\text { SD 0 } & 1,73 & 1,73 & 1,078 & 1,078 & 0 & 52,64 \% & 23,75 \% & 23,61 \% \\ \text { SD I } & 1,73 & 1,085 & 1,078 & 0,433 & 0,645 & 51,85 \% & 33,47 \% & 14,68 \% \\ \text { "Chance" I } & 1,622 & 1,622 & 1,078 & 1,078 & 0 & 50,03 \% & 24,72 \% & 25,25 \% \\ \text { "Chance" II } & 0,921 & 1,603 & 0,403 & 1,085 & -0,682 & & & \\ \text { Implícito I } & 1,9 & 1,9 & 1 & 1 & 0 & 58,41 \% & 22,11 \% & 19,49 \% \\ \text { Implícito II } & 1,9 & 1,303 & 1 & 0,403 & 0,597 & 59,56 \% & 29,03 \% & 11,41 \%\end{array}$

Coritiba 0x0 América MG

\begin{tabular}{|c|c|c|c|c|c|c|c|c|}
\hline SD 0 & 1,539 & 1,539 & 0,924 & 0,924 & 0 & $51,67 \%$ & $25,68 \%$ & $22,65 \%$ \\
\hline SD I & 1,539 & 1,27 & 0,924 & 0,655 & 0,269 & $51,31 \%$ & $29,49 \%$ & $19,20 \%$ \\
\hline "Chance" I & 1,562 & 1,562 & 0,866 & 0,866 & 0 & $53,71 \%$ & $25,45 \%$ & 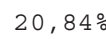 \\
\hline "Chance" II & 0,931 & 1,613 & 0,116 & 0,798 & $-0,682$ & & & \\
\hline Implícito I & 2 & 2 & 1,2 & 1,2 & 0 & $55,91 \%$ & $21,55 \%$ & $22,54 \stackrel{\circ}{\circ}$ \\
\hline Implícito II & 2 & 1,208 & 1,2 & 0,408 & 0,792 & $56,56 \%$ & $30,94 \%$ & $12,50 \%$ \\
\hline Goiás $3 \times 0 \mathrm{Br}$ & & & & & & & & \\
\hline SD 0 & 1,496 & 1,496 & 0,939 & 0,939 & 0 & $50,18 \%$ & $26,12 \%$ & $23,69 \%$ \\
\hline SD I & 1,496 & 1,337 & 0,939 & 0,78 & 0,159 & $49,90 \%$ & $28,27 \%$ & 21,82 웅 \\
\hline "Chance" I & 1,377 & 1,377 & 0,991 & 0,991 & 0 & $45,74 \%$ & $27,23 \%$ & $27,03 \%$ \\
\hline "Chance" II & 0,804 & 1,486 & 0,362 & 1,044 & $-0,682$ & & & \\
\hline Implícito I & 1,6 & 1,6 & 1,9 & 1,9 & 0 & $32,85 \%$ & $22,04 \%$ & $45,11 \%$ \\
\hline Implícito II & 1,6 & 0,508 & 1,9 & 0,808 & 1,092 & $21,32 \%$ & $39,01 \%$ & $39,67 \%$ \\
\hline
\end{tabular}


Flamengo 2x1 Goiás

$\begin{array}{lrrrrrrrr}\text { SD 0 } & 1,753 & 1,753 & 1,051 & 1,051 & 0 & 53,83 \% & 23,54 \% & 22,64 \% \\ \text { SD I } & 1,753 & 1,237 & 1,051 & 0,535 & 0,516 & 53,69 \% & 30,24 \% & 16,06 \% \\ \text { "Chance" I } & 1,934 & 1,934 & 0,984 & 0,984 & 0 & 59,54 \% & 21,75 \% & 18,71 \% \\ \text { "Chance" II } & 2,372 & 1,864 & 1,566 & 1,058 & 0,508 & 56,21 \% & 22,53 \% & 21,26 \% \\ \text { Implícito I } & 1,2 & 1,2 & 1,4 & 1,4 & 0 & 32,21 \% & 26,23 \% & 41,56 \% \\ \text { Implícito II } & 1,2 & 0,807 & 1,4 & 1,007 & 0,393 & 28,32 \% & 32,49 \% & 39,19 \%\end{array}$

Juventude $1 \times 2$ Santos

$\begin{array}{lrrrrrrrr}\text { SD 0 } & 1,101 & 1,101 & 0,9 & 0,9 & 0 & 39,94 \% & 30,62 \% & 29,44 \% \\ \text { SD I } & 1,101 & 0,903 & 0,9 & 0,703 & 0,198 & 38,24 \% & 34,98 \% & 26,78 \% \\ \text { "Chance" I } & 1,08 & 1,08 & 0,915 & 0,915 & 0 & 38,96 \% & 30,75 \% & 30,29 \% \\ \text { "Chance" II } & 1,618 & 1,111 & 1,464 & 0,957 & 0,508 & 38,91 \% & 30,12 \% & 30,97 \% \\ \text { Implícito I } & 1,9 & 1,9 & 1,9 & 1,9 & 0 & 39,35 \% & 21,29 \% & 39,35 \% \\ \text { Implícito II } & 1,9 & 0,508 & 1,9 & 0,508 & 1,392 & 26,91 \% & 46,17 \% & 26,91 \%\end{array}$

Implícito II

$1,90,508$

$1,90,508$

$1,392 \quad 26,91 \% \quad 46,17 \% \quad 26,91 \%$

Sport 1x0 Bragantino

\begin{tabular}{|c|c|c|c|c|c|c|c|c|}
\hline $\mathrm{SD} \quad 0$ & 1,995 & 1,995 & 0,516 & 0,516 & 0 & $72,59 \%$ & $18,89 \%$ & $8,52 \%$ \\
\hline SD I & 1,995 & 2,389 & 0,516 & 0,91 & $-0,394$ & & & \\
\hline "Chance" I & 2,065 & 2,065 & 0,687 & 0,687 & 0 & $69,54 \%$ & $19,20 \%$ & $11,26 \%$ \\
\hline "Chance" II & 2,479 & 1,971 & 1,074 & 0,567 & 0,508 & $70,80 \%$ & $19,53 \%$ & $9,67 \%$ \\
\hline Implícito I & 2 & 2 & 0,8 & 0,8 & 0 & $65,44 \%$ & $20,47 \%$ & $14,09 \%$ \\
\hline Implícito II & 2 & 1,608 & 0,8 & 0,408 & 0,392 & $67,41 \%$ & $23,60 \%$ & $8,99 \%$ \\
\hline Vitória $3 \times 0$ América & $\mathrm{RN}$ & & & & & & & \\
\hline SD 0 & 2,358 & 2,358 & 1,234 & 1,234 & 0 & $62,38 \%$ & $18,87 \%$ & $18,75 \%$ \\
\hline SD I & 2,358 & 2,988 & 1,234 & 1,864 & $-0,63$ & & & \\
\hline "Chance" I & 2,456 & 2,456 & 1,309 & 1,309 & 0 & $62,59 \%$ & $18,38 \%$ & $19,02 \%$ \\
\hline "Chance" II & 2,771 & 2,263 & 1,877 & 1,37 & 0,508 & $57,59 \%$ & $19,86 \%$ & $22,54 \%$ \\
\hline Implícito I & 1,6 & 1,6 & 0,8 & 0,8 & 0 & $56,37 \%$ & $24,97 \%$ & $18,65 \%$ \\
\hline Implícito II & 1,6 & 1,503 & 0,8 & 0,703 & 0,097 & $56,47 \%$ & $26,12 \%$ & $17,42 \%$ \\
\hline
\end{tabular}

Implícito II

$1,6 \quad 1,503 \quad 0,8 \quad 0,703$

$0,097 \quad 56,47 \% \quad 26,12 \% \quad 17,42 \%$

Atlético MG $1 \times 1$ Vasco

$\begin{array}{lrrrrrrrr}\text { SD 0 } & 1,404 & 1,404 & 1,427 & 1,427 & 0 & 36,92 \% & 25,12 \% & 37,96 \% \\ \text { SD I } & 1,404 & 0,581 & 1,427 & 0,604 & 0,823 & 28,14 \% & 42,26 \% & 29,60 \% \\ \text { "Chance" I } & 1,29 & 1,29 & 1,408 & 1,408 & 0 & 34,39 \% & 25,78 \% & 39,83 \% \\ \text { "Chance" II } & 1,859 & 1,351 & 1,954 & 1,446 & 0,508 & 35,23 \% & 25,26 \% & 39,51 \% \\ \text { Implícito I } & 1,4 & 1,4 & 1,4 & 1,4 & 0 & 37,36 \% & 25,28 \% & 37,36 \%\end{array}$

Implícito II

$1,4 \quad 1,008 \quad 1,4 \quad 1,008$

$0,392 \quad 34,65 \% 30,70 \% \quad 34,65 \%$

Juventude 1x2 Internacional

$\begin{array}{lrrrrrrrr}\text { SD 0 } & 1,035 & 1,035 & 0,772 & 0,772 & 0 & 40,95 \% & 32,39 \% & 26,65 \% \\ \text { SD I } & 1,035 & 0,611 & 0,772 & 0,348 & 0,424 & 35,47 \% & 46,92 \% & 17,61 \% \\ \text { "Chance" I } & 0,98 & 0,98 & 0,834 & 0,834 & 0 & 37,68 \% & 32,60 \% & 29,72 \% \\ \text { "Chance" II } & 1,544 & 1,013 & 1,339 & 0,808 & 0,531 & 39,37 \% & 32,40 \% & 28,22 \% \\ \text { Implícito I } & 1,6 & 1,6 & 1,5 & 1,5 & 0 & 40,26 \% & 23,82 \% & 35,92 \% \\ \text { Implícito II } & 1,6 & 0,803 & 1,5 & 0,703 & 0,797 & 34,63 \% & 36,59 \% & 28,78 \%\end{array}$


Cruzeiro 4x0 Coritiba

$\begin{array}{lrrrrrrrr}\text { SD 0 } & 1,388 & 1,388 & 0,514 & 0,514 & 0 & 58,67 \% & 27,63 \% & 13,70 \% \\ \text { SD I } & 1,388 & 1,167 & 0,514 & 0,293 & 0,221 & 58,86 \% & 31,87 \% & 9,27 \% \\ \text { "Chance" I } & 1,132 & 1,132 & 0,747 & 0,747 & 0 & 44,67 \% & 31,20 \% & 24,13 \% \\ \text { "Chance" II } & 1,694 & 1,164 & 1,126 & 0,595 & 0,531 & 49,72 \% & 31,38 \% & 18,90 \% \\ \text { Implícito I } & 1,4 & 1,4 & 1,1 & 1,1 & 0 & 43,80 \% & 26,63 \% & 29,56 \% \\ \text { Implícito II } & 1,4 & 1,203 & 1,1 & 0,903 & 0,197 & 42,93 \% & 29,46 \% & 27,61 \%\end{array}$

Paraná 2x1 Vasco

$\begin{array}{lrrrrrrrr}\text { SD 0 } & 0,948 & 0,948 & 1,415 & 1,415 & 0 & 25,23 \% & 26,96 \% & 47,81 \% \\ \text { SD I } & 0,948 & 0,546 & 1,415 & 1,013 & 0,402 & 19,47 \% & 34,38 \% & 46,15 \% \\ \text { "Chance" I } & 0,886 & 0,886 & 1,335 & 1,335 & 0 & 24,84 \% & 28,02 \% & 47,14 \% \\ \text { "Chance" II } & 1,412 & 0,881 & 1,901 & 1,37 & 0,531 & 24,14 \% & 27,63 \% & 48,23 \% \\ \text { Implícito I } & 1 & 1 & 1,5 & 1,5 & 0 & 25,22 \% & 25,98 \% & 48,79 \% \\ \text { Implícito II } & 1 & 0,908 & 1,5 & 1,408 & 0,092 & 24,26 \% & 27,13 \% & 48,61 \%\end{array}$

Implícito II

Ponte Preta 4x5 Corinthians

$\begin{array}{lrrrrrrrr}\text { SD 0 } & 0,721 & 0,721 & 1,157 & 1,157 & 0 & 22,92 \% & 30,96 \% & 46,12 \% \\ \text { SD I } & 0,721 & 0,879 & 1,157 & 1,315 & -0,158 & & & \\ \text { "Chance" I } & 0,706 & 0,706 & 1,15 & 1,15 & 0 & 22,57 \% & 31,15 \% & 46,28 \% \\ \text { "Chance" II } & 1,209 & 0,679 & 1,683 & 1,152 & 0,531 & 21,67 \% & 31,24 \% & 47,09 \% \\ \text { Implícito I } & 1 & 1 & 1,9 & 1,9 & 0 & 19,49 \% & 22,11 \% & 58,41 \%\end{array}$

Implícito I

$0,374 \quad 15,15 \% \quad 25,68 \% \quad 59,17 \%$

Santos 4x1 Flamengo

$\begin{array}{lrrrrrrrr}\text { SD 0 } & 2,147 & 2,147 & 0,945 & 0,945 & 0 & 64,87 \% & 19,63 \% & 15,50 \% \\ \text { SD I } & 2,147 & 2,251 & 0,945 & 1,048 & -0,103 & & & \\ \text { "Chance" I } & 2,311 & 2,311 & 1,036 & 1,036 & 0 & 65,90 \% & 18,55 \% & 15,55 \% \\ \text { "Chance" II } & 2,672 & 2,142 & 1,561 & 1,03 & 0,531 & 62,75 \% & 19,99 \% & 17,25 \% \\ \text { Implícito I } & 1,3 & 1,3 & 0,9 & 0,9 & 0 & 45,81 \% & 28,37 \% & 25,82 \% \\ \text { Implícito II } & 1,3 & 1,508 & 0,9 & 1,108 & -0,208 & & & \end{array}$

Botafogo 2x0 América RN

\begin{tabular}{|c|c|c|c|c|c|c|c|c|}
\hline SD 0 & 2,213 & 2,213 & 0,896 & 0,896 & 0 & $67,30 \%$ & $18,80 \%$ & $13,90 \%$ \\
\hline SD I & 2,213 & 1,706 & 0,896 & 0,389 & 0,507 & $70,19 \%$ & $21,93 \%$ & $7,87 \%$ \\
\hline "Chance" I & 2,386 & 2,386 & 0,935 & 0,935 & 0 & $69,54 \%$ & $17,44 \%$ & $13,01 \%$ \\
\hline "Chance" II & 2,747 & 2,217 & 1,423 & 0,892 & 0,531 & $67,45 \%$ & $18,75 \%$ & $13,79 \%$ \\
\hline Implícito I & 2 & 2 & 0,9 & 0,9 & 0 & $62,98 \%$ & $20,86 \%$ & $16,16 \%$ \\
\hline Implícito II & 2 & 1,508 & 0,9 & 0,408 & 0,492 & $64,97 \%$ & $25,27 \%$ & $9,76 \%$ \\
\hline Palmeiras $3 \times 2$ & Vitória BA & & & & & & & \\
\hline SD 0 & 0,865 & 0,865 & 0,363 & 0,363 & 0 & $45,96 \%$ & $39,23 \%$ & $14,81 \%$ \\
\hline SD I & 0,865 & 0,212 & 0,363 & $-0,29$ & 0,653 & & & \\
\hline "Chance" I & 1,558 & 1,558 & 0,612 & 0,612 & 0 & $60,36 \%$ & $25,19 \%$ & $14,45 \%$ \\
\hline "Chance" II & 2,156 & 1,626 & 0,917 & 0,386 & 0,531 & $68,46 \%$ & $23,18 \%$ & $8,36 \%$ \\
\hline Implícito I & 1,7 & 1,7 & 1,4 & 1,4 & 0 & $44,69 \%$ & $23,57 \%$ & $31,74 \%$ \\
\hline Implícito II & 1,7 & 0,903 & 1,4 & 0,603 & 0,797 & $40,73 \%$ & $36,02 \%$ & $23,25 \%$ \\
\hline
\end{tabular}


São Paulo $2 \times 7$ Portuguesa

$\begin{array}{lrrrrrrrr}\text { SD 0 } & 1,685 & 1,685 & 1,512 & 1,512 & 0 & 42,02 \% & 23,35 \% & 34,63 \% \\ \text { SD I } & 1,685 & 1,993 & 1,512 & 1,82 & -0,308 & & & \\ \text { "Chance" I } & 1,821 & 1,821 & 1,672 & 1,672 & 0 & 41,93 \% & 22,25 \% & 35,83 \% \\ \text { "Chance" II } & 2,311 & 1,781 & 2,202 & 1,672 & 0,531 & 41,04 \% & 22,42 \% & 36,54 \% \\ \text { Implícito I } & 1,5 & 1,5 & 2 & 2 & 0 & 29,03 \% & 21,62 \% & 49,36 \% \\ \text { Implícito II } & 1,5 & 0,303 & 2 & 0,803 & 1,197 & 13,10 \% & 41,66 \% & 45,23 \%\end{array}$

Guarani 2x2 Goiás

$\begin{array}{lrrrrrrrr}\text { SD 0 } & 1,986 & 1,986 & 0,88 & 0,88 & 0 & 63,18 \% & 20,93 \% & 15,89 \% \\ \text { SD I } & 1,986 & 1,663 & 0,88 & 0,557 & 0,323 & 64,46 \% & 23,51 \% & 12,04 \% \\ \text { "Chance" I } & 2,191 & 2,191 & 0,906 & 0,906 & 0 & 66,65 \% & 19,05 \% & 14,30 \% \\ \text { "Chance" II } & 2,585 & 2,055 & 1,452 & 0,921 & 0,531 & 63,60 \% & 20,40 \% & 15,99 \% \\ \text { Implícito I } & 2 & 2 & 0,8 & 0,8 & 0 & 65,44 \% & 20,47 \% & 14,09 \% \\ \text { Implícito II } & 2 & 1,608 & 0,8 & 0,408 & 0,392 & 67,41 \% & 23,60 \% & 8,99 \%\end{array}$

América MG 0x2 Atlético MG

$\begin{array}{lrrrrrrrr}\text { SD 0 } & 1,297 & 1,297 & 1,716 & 1,716 & 0 & 29,03 \% & 23,67 \% & 47,30 \% \\ \text { SD I } & 1,297 & 1,136 & 1,716 & 1,555 & 0,161 & 27,79 \% & 25,21 \% & 46,99 \% \\ \text { "Chance" I } & 1,412 & 1,412 & 1,9 & 1,9 & 0 & 28,68 \% & 22,30 \% & 49,02 \% \\ \text { "Chance" II } & 1,987 & 1,457 & 2,384 & 1,853 & 0,531 & 30,43 \% & 22,53 \% & 47,03 \% \\ \text { Implícito I } & 1,4 & 1,4 & 1,4 & 1,4 & 0 & 37,36 \% & 25,28 \% & 37,36 \% \\ \text { Implícito II } & 1,4 & 1,008 & 1,4 & 1,008 & 0,392 & 34,65 \% & 30,70 \% & 34,65 \%\end{array}$

Sport 5xo Grêmio

$\begin{array}{lrrrrrrrr}\text { SD 0 } & 2,029 & 2,029 & 0,798 & 0,798 & 0 & 66,08 \% & 20,16 \% & 13,76 \% \\ \text { SD I } & 2,029 & 1,737 & 0,798 & 0,506 & 0,292 & 67,57 \% & 22,21 \% & 10,22 \% \\ \text { "Chance" I } & 2,052 & 2,052 & 0,839 & 0,839 & 0 & 65,52 \% & 20,11 \% & 14,37 \% \\ \text { "Chance" II } & 2,487 & 1,957 & 1,3 & 0,77 & 0,531 & 65,31 \% & 20,80 \% & 13,89 \% \\ \text { Implícito I } & 2 & 2 & 1,4 & 1,4 & 0 & 51,48 \% & 21,66 \% & 26,86 \% \\ \text { Implícito II } & 2 & 1,126 & 1,4 & 0,526 & 0,874 & 50,48 \% & 32,32 \% & 17,20 \%\end{array}$

Implícito II

$0,874 \quad 50,48 \% \quad 32,32 \% \quad 17,20 \%$

Palmeiras 4xo Bragantino

$\begin{array}{lrrrrrrrr}\text { SD 0 } & 2,156 & 2,156 & 0,274 & 0,274 & 0 & 81,54 \% & 14,82 \% & 3,64 \% \\ \text { SD I } & 2,156 & 2,959 & 0,274 & 1,077 & -0,803 & & & \\ \text { "Chance" I } & 2,238 & 2,238 & 0,554 & 0,554 & 0 & 75,91 \% & 16,44 \% & 7,64 \% \\ \text { "Chance" II } & 2,715 & 2,104 & 0,921 & 0,31 & 0,611 & 79,82 \% & 15,81 \% & 4,37 \% \\ \text { Implícito I } & 2 & 2 & 0,7 & 0,7 & 0 & 67,95 \% & 19,98 \% & 12,07 \% \\ \text { Implícito II } & 2 & 1,603 & 0,7 & 0,303 & 0,397 & 70,41 \% & 23,02 \% & 6,57 \%\end{array}$

Implícito II $\quad 2 \quad 1,603 \quad 0,7 \quad 0,303 \quad 0,397 \quad 70,41 \% \quad 23,02 \% \quad 6,57 \%$

Goiás 2xo Botafogo

$\begin{array}{lrrrrrrrr}\text { SD 0 } & 1,258 & 1,258 & 1,367 & 1,367 & 0 & 34,36 \% & 26,20 \% & 39,44 \% \\ \text { SD I } & 1,258 & 0,464 & 1,367 & 0,572 & 0,794 & 23,60 \% & 45,55 \% & 30,85 \% \\ \text { "Chance" I } & 1,141 & 1,141 & 1,421 & 1,421 & 0 & 30,28 \% & 26,31 \% & 43,42 \% \\ \text { "Chance" II } & 1,801 & 1,189 & 2,085 & 1,473 & 0,611 & 30,58 \% & 25,72 \% & 43,70 \% \\ \text { Implícito I } & 2 & 2 & 1,7 & 1,7 & 0 & 45,29 \% & 21,38 \% & 33,33 \% \\ \text { Implícito II } & 2 & 0,826 & 1,7 & 0,526 & 1,174 & 39,89 \% & 38,40 \% & 21,71 \%\end{array}$


América MG $1 \times 3$ São Paulo

$\begin{array}{lrrrrrrrr}\text { SD 0 } & 1,256 & 1,256 & 1,539 & 1,539 & 0 & 31,09 \% & 25,02 \% & 43,89 \% \\ \text { SD I } & 1,256 & 0,727 & 1,539 & 1,01 & 0,529 & 25,64 \% & 33,11 \% & 41,26 \% \\ \text { "Chance" I } & 1,132 & 1,132 & 1,455 & 1,455 & 0 & 29,42 \% & 26,06 \% & 44,53 \% \\ \text { "Chance" II } & 1,794 & 1,182 & 2,123 & 1,512 & 0,611 & 29,71 \% & 25,44 \% & 44,85 \% \\ \text { Implícito I } & 2 & 2 & 1,6 & 1,6 & 0 & 47,29 \% & 21,53 \% & 31,18 \% \\ \text { Implícito II } & 2 & 0,703 & 1,6 & 0,303 & 1,297 & 40,91 \% & 44,80 \% & 14,28 \%\end{array}$

Vasco $1 \times 1$ Juventude

$\begin{array}{lrrrrrrrr}\text { SD 0 } & 1,346 & 1,346 & 0,421 & 0,421 & 0 & 60,25 \% & 28,23 \% & 11,52 \% \\ \text { SD I } & 1,346 & 1,254 & 0,421 & 0,329 & 0,092 & 60,41 \% & 29,93 \% & 9,66 \% \\ \text { "Chance" I } & 1,268 & 1,268 & 0,649 & 0,649 & 0 & 51,41 \% & 29,54 \% & 19,05 \% \\ \text { "Chance" II } & 1,946 & 1,334 & 1,07 & 0,458 & 0,611 & 58,81 \% & 28,50 \% & 12,69 \% \\ \text { Implícito I } & 1,6 & 1,6 & 1,4 & 1,4 & 0 & 42,31 \% & 24,17 \% & 33,52 \% \\ \text { Implícito II } & 1,6 & 0,807 & 1,4 & 0,607 & 0,793 & 37,10 \% & 37,76 \% & 25,14 \%\end{array}$

Implícito II

$1,6 \quad 0,807 \quad 1,4 \quad 0,607$

$0,793 \quad 37,10 \% \quad 37,76 \% \quad 25,14 \%$

Flamengo 1x3 Internacional

\begin{tabular}{|c|c|c|c|c|c|c|c|c|}
\hline $\mathrm{SD} 0$ & 1,368 & 1,368 & 1,489 & 1,489 & 0 & $34,81 \%$ & $24,94 \%$ & $40,25 \%$ \\
\hline $\mathrm{SD} I$ & 1,368 & 1,065 & 1,489 & 1,186 & 0,303 & $32,65 \%$ & $28,67 \%$ & $38,68 \%$ \\
\hline "Chance" I & 1,311 & 1,311 & 1,395 & 1,395 & 0 & $35,18 \%$ & $25,76 \%$ & $39,06 \%$ \\
\hline "Chance" II & 1,977 & 1,366 & 2,08 & 1,468 & 0,611 & $35,15 \%$ & $25,07 \%$ & $39,78 \%$ \\
\hline Implícito I & 1,7 & 1,7 & 2 & 2 & 0 & $33,33 \%$ & $21,38 \%$ & $45,29 \%$ \\
\hline Implícito II & 1,7 & 0,408 & 2 & 0,708 & 1,292 & $18,83 \%$ & $42,93 \%$ & $38,24 \%$ \\
\hline
\end{tabular}

Implícito II

$\begin{array}{llll}1,7 & 0,408 & 2 & 0,708\end{array}$

$1,292 \quad 18,83 \% \quad 42,93 \% \quad 38,24 \%$

Portuguesa $2 \times 0$ sport

$\begin{array}{lrrrrrrrr}\text { SD 0 } & 1,761 & 1,761 & 1,226 & 1,226 & 0 & 49,94 \% & 23,43 \% & 26,63 \% \\ \text { SD I } & 1,761 & 1,201 & 1,226 & 0,666 & 0,561 & 48,94 \% & 30,54 \% & 20,53 \% \\ \text { "Chance" I } & 1,679 & 1,679 & 1,183 & 1,183 & 0 & 48,97 \% & 24,12 \% & 26,92 \% \\ \text { "Chance" II } & 2,356 & 1,745 & 1,831 & 1,22 & 0,611 & 49,69 \% & 23,56 \% & 26,74 \% \\ \text { Implícito I } & 1,9 & 1,9 & 1,7 & 1,7 & 0 & 43,13 \% & 21,83 \% & 35,04 \% \\ \text { Implícito II } & 1,9 & 0,708 & 1,7 & 0,508 & 1,192 & 35,66 \% & 41,30 \% & 23,04 \%\end{array}$

Implícito II

$1,9 \quad 0,708 \quad 1,7 \quad 0,508$

$1,192 \quad 35,66 \% \quad 41,30 \% \quad 23,04 \%$

Corinthians 1x2 Cruzeiro

$\begin{array}{lrrrrrrrr}\text { SD 0 } & 1,572 & 1,572 & 0,7 & 0,7 & 0 & 58,32 \% & 25,19 \% & 16,50 \% \\ \text { SD I } & 1,572 & 1,367 & 0,7 & 0,495 & 0,205 & 58,64 \% & 27,97 \% & 13,39 \% \\ \text { "Chance" I } & 1,571 & 1,571 & 0,792 & 0,792 & 0 & 55,86 \% & 25,31 \% & 18,83 \% \\ \text { "Chance" II } & 2,19 & 1,579 & 1,299 & 0,687 & 0,611 & 58,84 \% & 25,07 \% & 16,09 \% \\ \text { Implícito I } & 1,4 & 1,4 & 1 & 1 & 0 & 46,15 \% & 26,97 \% & 26,88 \% \\ \text { Implícito II } & 1,4 & 1,207 & 1 & 0,807 & 0,193 & 45,43 \% & 29,87 \% & 24,70 \%\end{array}$

Implícito II

$1,4 \quad 1,207$

$0,193 \quad 45,43 \% \quad 29,87 \% \quad 24,70 \%$

Grêmio 1x0 Ponte Preta

$\begin{array}{lrrrrrrrr}\text { SD 0 } & 1,711 & 1,711 & 1,208 & 1,208 & 0 & 49,17 \% & 23,83 \% & 27,00 \% \\ \text { SD I } & 1,711 & 0,137 & 1,208 & -0,365 & 1,574 & & & \\ \text { "Chance" I } & 1,628 & 1,628 & 1,069 & 1,069 & 0 & 50,39 \% & 24,68 \% & 24,93 \% \\ \text { "Chance" II } & 2,226 & 1,615 & 1,78 & 1,168 & 0,611 & 47,74 \% & 24,66 \% & 27,60 \% \\ \text { Implícito I } & 2 & 2 & 1,5 & 1,5 & 0 & 49,36 \% & 21,62 \% & 29,03 \% \\ \text { Implícito II } & 2 & 0,908 & 1,5 & 0,408 & 1,092 & 46,29 \% & 37,72 \% & 15,99 \%\end{array}$


Atlético PR 4xo Guarani

$\begin{array}{lrrrrrrrr}\text { SD 0 } & 1,554 & 1,554 & 1,365 & 1,365 & 0 & 41,92 \% & 24,56 \% & 33,52 \% \\ \text { SD I } & 1,554 & 1,609 & 1,365 & 1,42 & -0,055 & & & \\ \text { "Chance" I } & 1,533 & 1,533 & 1,277 & 1,277 & 0 & 43,27 \% & 25,00 \% & 31,73 \% \\ \text { "Chance" II } & 2,17 & 1,559 & 1,922 & 1,31 & 0,611 & 43,20 \% & 24,71 \% & 32,09 \% \\ \text { Implícito I } & 1,4 & 1,4 & 1,9 & 1,9 & 0 & 28,41 \% & 22,31 \% & 49,28 \% \\ \text { Implícito II } & 1,4 & 0,403 & 1,9 & 0,903 & 0,997 & 15,85 \% & 37,90 \% & 46,25 \%\end{array}$

Juventude $3 \times 6$ Palmeiras

$\begin{array}{lrrrrrrrr}\text { SD 0 } & 0,922 & 0,922 & 1,015 & 1,015 & 0 & 31,83 \% & 31,41 \% & 36,76 \% \\ \text { SD I } & 0,922 & 1,315 & 1,015 & 1,407 & -0,392 & & & \\ \text { "Chance" I } & 0,894 & 0,894 & 0,987 & 0,987 & 0 & 31,52 \% & 31,97 \% & 36,51 \% \\ \text { "Chance" II } & 1,434 & 0,895 & 1,582 & 1,043 & 0,539 & 30,44 \% & 31,32 \% & 38,24 \% \\ \text { Implícito I } & 1,2 & 1,2 & 1,9 & 1,9 & 0 & 23,94 \% & 22,35 \% & 53,72 \% \\ \text { Implícito II } & 1,2 & 0,508 & 1,9 & 1,208 & 0,692 & 15,59 \% & 30,82 \% & 53,59 \%\end{array}$

São Paulo 0x0 Flamengo

$\begin{array}{lrrrrrrrr}\text { SD 0 } & 2,269 & 2,269 & 1,057 & 1,057 & 0 & 64,63 \% & 18,98 \% & 16,38 \% \\ \text { SD I } & 2,269 & 2,095 & 1,057 & 0,883 & 0,174 & 65,33 \% & 19,86 \% & 14,81 \% \\ \text { "Chance" I } & 2,345 & 2,345 & 1,077 & 1,077 & 0 & 65,59 \% & 18,43 \% & 15,97 \% \\ \text { "Chance" II } & 2,704 & 2,165 & 1,613 & 1,074 & 0,539 & 62,21 \% & 19,92 \% & 17,85 \% \\ \text { Implícito I } & 1,4 & 1,4 & 1 & 1 & 0 & 46,15 \% & 26,97 \% & 26,88 \% \\ \text { Implícito II } & 1,4 & 1,303 & 1 & 0,903 & 0,097 & 45,82 \% & 28,33 \% & 25,85 \%\end{array}$

Implícito II

$1,4 \quad 1,303 \quad 1 \quad 0,903$

$0,097 \quad 45,82 \% \quad 28,33 \% 25,85 \%$

Corinthians 0x2 Santos

\begin{tabular}{|c|c|c|c|c|c|c|c|c|}
\hline SD 0 & 1,585 & 1,585 & 1,2 & 1,2 & 0 & $46,28 \%$ & $24,83 \%$ & $28,89 \%$ \\
\hline SD I & 1,585 & 1,528 & 1,2 & 1,142 & 0,058 & $46,15 \%$ & $25,43 \%$ & $28,43 \%$ \\
\hline "Chance" I & 1,564 & 1,564 & 1,18 & 1,18 & 0 & $46,21 \%$ & $25,04 \%$ & $28,76 \%$ \\
\hline "Chance" II & 2,113 & 1,574 & 1,779 & 1,24 & 0,539 & $45,12 \%$ & $24,81 \%$ & $30,06 \%$ \\
\hline Implícito I & 2,1 & 2,1 & 2 & 2 & 0 & $41,70 \%$ & $20,40 \%$ & $37,89 \%$ \\
\hline Implícito II & 2,1 & 0,526 & 2 & 0,426 & 1,574 & $29,54 \%$ & $47,76 \%$ & $22,70 \%$ \\
\hline
\end{tabular}

Implícito II

$2,1 \quad 0,526$

$1,574 \quad 29,54 \% \quad 47,76 \% \quad 22,70 \%$

Botafogo 0x2 Vasco

\begin{tabular}{|c|c|c|c|c|c|c|c|c|}
\hline SD 0 & 1,175 & 1,175 & 1,43 & 1,43 & 0 & $30,99 \%$ & $26,11 \%$ & $42,90 \%$ \\
\hline SD I & 1,175 & 0,811 & 1,43 & 1,066 & 0,364 & $27,33 \%$ & $31,69 \%$ & $40,99 \%$ \\
\hline "Chance" I & 1,297 & 1,297 & 1,634 & 1,634 & 0 & $30,43 \%$ & $24,25 \%$ & $45,33 \%$ \\
\hline "Chance" II & 1,887 & 1,348 & 2,16 & 1,621 & 0,539 & $31,88 \%$ & $24,20 \%$ & $43,92 \%$ \\
\hline Implícito I & 0,9 & 0,9 & 1,8 & 1,8 & 0 & $18,50 \%$ & $22,90 \%$ & $58,61 \%$ \\
\hline
\end{tabular}

Implícito II $\quad 0,9 \quad 0,503 \quad 1,8 \quad 1,403 \quad 0,397 \quad 13,23 \% \quad 27,37 \%$

Portuguesa $1 \times 0$ Ponte Preta

$\begin{array}{lrrrrrrrr}\text { SD 0 } & 2,194 & 2,194 & 0,592 & 0,592 & 0 & 74,25 \% & 17,18 \% & 8,56 \% \\ \text { SD I } & 2,194 & 0,297 & 0,592 & -1,305 & 1,897 & & \\ \text { "Chance" I } & 2,378 & 2,378 & 0,7 & 0,7 & 0 & 74,75 \% & 16,12 \% & 9,13 \% \\ \text { "Chance" II } & 2,697 & 2,158 & 1,105 & 0,566 & 0,539 & 74,26 \% & 17,38 \% & 8,35 \% \\ \text { Implícito I } & 2,2 & 2,2 & 1 & 1 & 0 & 64,62 \% & 19,36 \% & 16,01 \% \\ \text { Implícito II } & 2,2 & 1,526 & 1 & 0,326 & 0,674 & 67,88 \% & 24,53 \% & 7,59 \%\end{array}$


Atlético PR 3x2 Bragantino

$\begin{array}{lrrrrrrrr}\text { SD 0 } & 1,853 & 1,853 & 0,669 & 0,669 & 0 & 65,70 \% & 21,53 \% & 12,77 \% \\ \text { SD I } & 1,853 & 2,204 & 0,669 & 1,021 & -0,352 & & & \\ \text { "Chance" I } & 1,802 & 1,802 & 0,718 & 0,718 & 0 & 63,30 \% & 22,35 \% & 14,35 \% \\ \text { "Chance" II } & 2,337 & 1,798 & 1,169 & 0,63 & 0,539 & 65,53 \% & 22,03 \% & 12,44 \% \\ \text { Implícito I } & 2,1 & 2,1 & 0,8 & 0,8 & 0 & 67,44 \% & 19,43 \% & 13,13 \% \\ \text { Implícito II } & 2,1 & 1,608 & 0,8 & 0,308 & 0,492 & 70,37 \% & 22,96 \% & 6,66 \%\end{array}$

Guarani 0x1 Coritiba

$\begin{array}{lrrrrrrrr}\text { SD 0 } & 1,834 & 1,834 & 0,85 & 0,85 & 0 & 60,63 \% & 22,42 \% & 16,95 \% \\ \text { SD I } & 1,834 & 1,76 & 0,85 & 0,776 & 0,074 & 60,84 \% & 23,04 \% & 16,13 \% \\ \text { "Chance" I } & 1,623 & 1,623 & 0,976 & 0,976 & 0 & 52,52 \% & 24,78 \% & 22,70 \% \\ \text { "Chance" II } & 2,179 & 1,64 & 1,523 & 0,984 & 0,539 & 52,72 \% & 24,61 \% & 22,67 \% \\ \text { Implícito I } & 1,8 & 1,8 & 1,5 & 1,5 & 0 & 44,92 \% & 22,77 \% & 32,32 \% \\ \text { Implícito II } & 1,8 & 0,803 & 1,5 & 0,503 & 0,997 & 39,60 \% & 39,20 \% & 21,19 \%\end{array}$

Atlético MG 3x0 Paraná

$\begin{array}{lrrrrrrrr}\text { SD 0 } & 1,87 & 1,87 & 0,892 & 0,892 & 0 & 60,38 \% & 22,15 \% & 17,47 \% \\ \text { SD I } & 1,87 & 1,426 & 0,892 & 0,448 & 0,444 & 61,64 \% & 26,84 \% & 11,52 \% \\ \text { "Chance" I } & 1,759 & 1,759 & 0,902 & 0,902 & 0 & 57,63 \% & 23,32 \% & 19,05 \% \\ \text { "Chance" II } & 2,271 & 1,732 & 1,406 & 0,867 & 0,539 & 57,87 \% & 23,56 \% & 18,57 \% \\ \text { Implícito I } & 1,8 & 1,8 & 1,4 & 1,4 & 0 & 47,02 \% & 22,95 \% & 30,03 \% \\ \text { Implícito II } & 1,8 & 0,903 & 1,4 & 0,503 & 0,897 & 43,41 \% & 36,99 \% & 19,60 \%\end{array}$

Sport 1x1 Cruzeiro

$\begin{array}{lrrrrrrrr}\text { SD 0 } & 1,492 & 1,492 & 0,718 & 0,718 & 0 & 55,78 \% & 26,27 \% & 17,95 \% \\ \text { SD I } & 1,492 & 1,567 & 0,718 & 0,793 & -0,075 & & & \\ \text { "Chance" I } & 1,515 & 1,515 & 0,807 & 0,807 & 0 & 54,02 \% & 26,00 \% & 19,98 \% \\ \text { "Chance" II } & 2,074 & 1,535 & 1,264 & 0,725 & 0,539 & 56,73 \% & 25,70 \% & 17,57 \% \\ \text { Implícito I } & 2 & 2 & 1,5 & 1,5 & 0 & 49,36 \% & 21,62 \% & 29,03 \% \\ \text { Implícito II } & 2 & 1,026 & 1,5 & 0,526 & 0,974 & 47,15 \% & 34,26 \% & 18,59 \%\end{array}$

Implícito II

$0,974 \quad 47,15 \% \quad 34,26 \% \quad 18,59 \%$

Grêmio 2x1 América MG

$\begin{array}{lrrrrrrrr}\text { SD 0 } & 1,701 & 1,701 & 1,186 & 1,186 & 0 & 49,43 \% & 23,94 \% & 26,63 \% \\ \text { SD I } & 1,701 & 1,448 & 1,186 & 0,934 & 0,253 & 49,06 \% & 26,64 \% & 24,30 \% \\ \text { "Chance" I } & 1,55 & 1,55 & 1,047 & 1,047 & 0 & 48,94 \% & 25,43 \% & 25,63 \% \\ \text { "Chance" II } & 2,143 & 1,604 & 1,663 & 1,124 & 0,539 & 48,50 \% & 24,82 \% & 26,68 \% \\ \text { Implícito I } & 2 & 2 & 0,9 & 0,9 & 0 & 62,98 \% & 20,86 \% & 16,16 \% \\ \text { Implícito II } & 2 & 1,508 & 0,9 & 0,408 & 0,492 & 64,97 \% & 25,27 \% & 9,76 \%\end{array}$

Vitória 2x0 Internacional

$\begin{array}{lrrrrrrrr}\text { SD 0 } & 1,734 & 1,734 & 1,459 & 1,459 & 0 & 44,25 \% & 23,23 \% & 32,52 \% \\ \text { SD I } & 1,734 & 1,824 & 1,459 & 1,549 & -0,09 & & & \\ \text { "Chance" I } & 1,513 & 1,513 & 1,538 & 1,538 & 0 & 37,41 \% & 24,07 \% & 38,52 \% \\ \text { "Chance" II } & 2,136 & 1,597 & 2,144 & 1,605 & 0,539 & 38,11 \% & 23,42 \% & 38,46 \% \\ \text { Implícito I } & 2 & 2 & 1,6 & 1,6 & 0 & 47,29 \% & 21,53 \% & 31,18 \% \\ \text { Implícito II } & 2 & 0,808 & 1,6 & 0,408 & 1,192 & 42,40 \% & 40,25 \% & 17,35 \%\end{array}$


América RN 2x1 Goiás

$\begin{array}{lrrrrrrrr}\text { SD 0 } & 1,511 & 1,511 & 1,262 & 1,262 & 0 & 43,06 \% & 25,20 \% & 31,74 \% \\ \text { SD I } & 1,511 & 1,265 & 1,262 & 1,016 & 0,246 & 42,05 \% & 28,22 \% & 29,74 \% \\ \text { "Chance" I } & 1,503 & 1,503 & 1,189 & 1,189 & 0 & 44,47 \% & 25,49 \% & 30,03 \% \\ \text { "Chance" II } & 2,079 & 1,54 & 1,863 & 1,324 & 0,539 & 42,44 \% & 24,80 \% & 32,76 \% \\ \text { Implícito I } & 1 & 1 & 1,4 & 1,4 & 0 & 26,88 \% & 26,97 \% & 46,15 \% \\ \text { Implícito II } & 1 & 0,903 & 1,4 & 1,303 & 0,097 & 25,85 \% & 28,33 \% & 45,82 \%\end{array}$

Santos $4 \times 0$ Ponte Preta

$\begin{array}{lrrrrrrrr}\text { SD 0 } & 2,048 & 2,048 & 0,348 & 0,348 & 0 & 77,93 \% & 16,85 \% & 5,22 \% \\ \text { SD I } & 2,048 & 1,014 & 0,348 & -0,685 & 1,034 & & & \\ \text { "Chance" I } & 2,189 & 2,189 & 0,612 & 0,612 & 0 & 73,68 \% & 17,38 \% & 8,94 \% \\ \text { "Chance" II } & 2,503 & 2,038 & 0,876 & 0,411 & 0,465 & 76,11 \% & 17,54 \% & 6,35 \% \\ \text { Implícito I } & 2 & 2 & 1 & 1 & 0 & 60,57 \% & 21,17 \% & 18,26 \% \\ \text { Implícito II } & 2 & 1,408 & 1 & 0,408 & 0,592 & 62,35 \% & 27,04 \% & 10,60 \%\end{array}$

Implícito II

Atlético PR 3x1 Botafogo

$\begin{array}{lrrrrrrrr}\text { SD 0 } & 1,508 & 1,508 & 1,214 & 1,214 & 0 & 44,04 \% & 25,38 \% & 30,58 \% \\ \text { SD I } & 1,508 & 1,06 & 1,214 & 0,766 & 0,448 & 41,91 \% & 32,09 \% & 26,00 \% \\ \text { "Chance" I } & 1,449 & 1,449 & 1,153 & 1,153 & 0 & 43,88 \% & 26,04 \% & 30,08 \% \\ \text { "Chance" II } & 1,918 & 1,453 & 1,608 & 1,143 & 0,465 & 44,23 \% & 26,04 \% & 29,73 \% \\ \text { Implícito I } & 1,4 & 1,4 & 1,3 & 1,3 & 0 & 39,41 \% & 25,78 \% & 34,81 \% \\ \text { Implícito II } & 1,4 & 1,003 & 1,3 & 0,903 & 0,397 & 36,81 \% & 31,72 \% & 31,47 \%\end{array}$

Implícito II $\quad 1,4 \quad 1,003 \quad 1,3 \quad 0,903 \quad 0,397 \quad 36,81 \% 31,72 \% 31,47 \%$

Juventude 1x0 América MG

$\begin{array}{lrrrrrrrr}\text { SD 0 } & 1,636 & 1,636 & 0,537 & 0,537 & 0 & 64,38 \% & 23,81 \% & 11,82 \% \\ \text { SD I } & 1,636 & 0,936 & 0,537 & -0,163 & 0,7 & & & \\ \text { "Chance" I } & 1,537 & 1,537 & 0,712 & 0,712 & 0 & 57,11 \% & 25,67 \% & 17,22 \% \\ \text { "Chance" II } & 2,068 & 1,603 & 1,025 & 0,56 & 0,465 & 62,94 \% & 24,38 \% & 12,68 \% \\ \text { Implícito I } & 1,5 & 1,5 & 1,2 & 1,2 & 0 & 44,15 \% & 25,48 \% & 30,37 \%\end{array}$

Implícito II $\quad 1,5 \quad 1,208 \quad 1,2 \quad 0,908 \quad 0,292 \quad 42,96 \% \quad 29,37 \% \quad 27,67 \%$

Vitória 1x1 Atlético MG

$\begin{array}{lrrrrrrrr}\text { SD 0 } & 2,063 & 2,063 & 1,396 & 1,396 & 0 & 52,92 \% & 21,24 \% & 25,84 \% \\ \text { SD I } & 2,063 & 2,301 & 1,396 & 1,635 & -0,238 & & & \\ \text { "Chance" I } & 1,978 & 1,978 & 1,453 & 1,453 & 0 & 49,85 \% & 21,78 \% & 28,36 \% \\ \text { "Chance" II } & 2,381 & 1,916 & 1,976 & 1,511 & 0,465 & 47,28 \% & 22,10 \% & 30,62 \% \\ \text { Implícito I } & 1,7 & 1,7 & 1,4 & 1,4 & 0 & 44,69 \% & 23,57 \% & 31,74 \% \\ \text { Implícito II } & 1,7 & 1,008 & 1,4 & 0,708 & 0,692 & 41,67 \% & 33,29 \% & 25,04 \%\end{array}$

Coritiba $3 \times 2$ Paraná

$\begin{array}{lrrrrrrrr}\text { SD 0 } & 1,249 & 1,249 & 1,121 & 1,121 & 0 & 39,22 \% & 27,80 \% & 32,98 \% \\ \text { SD I } & 1,249 & 1,323 & 1,121 & 1,195 & -0,074 & & & \\ \text { "Chance" I } & 1,381 & 1,381 & 1,087 & 1,087 & 0 & 43,59 \% & 26,85 \% & 29,56 \% \\ \text { "Chance" II } & 1,903 & 1,412 & 1,609 & 1,118 & 0,491 & 43,70 \% & 26,47 \% & 29,83 \% \\ \text { Implícito I } & 1,8 & 1,8 & 1,3 & 1,3 & 0 & 49,19 \% & 23,07 \% & 27,73 \% \\ \text { Implícito II } & 1,8 & 1,003 & 1,3 & 0,503 & 0,797 & 47,00 \% & 34,87 \% & 18,12 \%\end{array}$


América RN 1×3 Vasco

$\begin{array}{lrrrrrrrr}\text { SD 0 } & 1,091 & 1,091 & 1,615 & 1,615 & 0 & 25,69 \% & 24,77 \% & 49,54 \% \\ \text { SD I } & 1,091 & 1,078 & 1,615 & 1,602 & 0,013 & 25,58 \% & 24,90 \% & 49,53 \% \\ \text { "Chance" I } & 1,012 & 1,012 & 1,609 & 1,609 & 0 & 23,82 \% & 24,90 \% & 51,28 \% \\ \text { "Chance" II } & 1,52 & 1,029 & 2,18 & 1,689 & 0,491 & 23,05 \% & 24,13 \% & 52,82 \% \\ \text { Implícito I } & 1,4 & 1,4 & 2 & 2 & 0 & 26,86 \% & 21,66 \% & 51,48 \% \\ \text { Implícito II } & 1,4 & 0,526 & 2 & 1,126 & 0,874 & 17,20 \% & 32,32 \% & 50,48 \%\end{array}$

Vitória 1xo são Paulo

$\begin{array}{lrrrrrrrr}\text { SD 0 } & 1,896 & 1,896 & 1,511 & 1,511 & 0 & 46,85 \% & 22,21 \% & 30,94 \% \\ \text { SD I } & 1,896 & 1,979 & 1,511 & 1,594 & -0,083 & & & \\ \text { "Chance" I } & 1,741 & 1,741 & 1,583 & 1,583 & 0 & 41,89 \% & 22,86 \% & 35,25 \% \\ \text { "Chance" II } & 2,236 & 1,744 & 2,113 & 1,622 & 0,491 & 41,20 \% & 22,73 \% & 36,07 \% \\ \text { Implícito I } & 1,7 & 1,7 & 1,4 & 1,4 & 0 & 44,69 \% & 23,57 \% & 31,74 \% \\ \text { Implícito II } & 1,7 & 1,008 & 1,4 & 0,708 & 0,692 & 41,67 \% & 33,29 \% & 25,04 \%\end{array}$

Flamengo 3x2 Atlético MG

$\begin{array}{lrrrrrrrr}\text { SD 0 } & 1,624 & 1,624 & 1,471 & 1,471 & 0 & 41,42 \% & 23,80 \% & 34,79 \% \\ \text { SD I } & 1,624 & 1,33 & 1,471 & 1,177 & 0,294 & 40,21 \% & 26,86 \% & 32,93 \% \\ \text { "Chance" I } & 1,616 & 1,616 & 1,361 & 1,361 & 0 & 43,51 \% & 24,19 \% & 32,29 \% \\ \text { "Chance" II } & 2,098 & 1,607 & 1,916 & 1,425 & 0,491 & 41,96 \% & 24,04 \% & 34,00 \% \\ \text { Implícito I } & 1,4 & 1,4 & 1,5 & 1,5 & 0 & 35,40 \% & 24,74 \% & 39,86 \% \\ \text { Implícito II } & 1,4 & 0,803 & 1,5 & 0,903 & 0,597 & 30,25 \% & 33,92 \% & 35,83 \%\end{array}$

Botafogo 2x1 Grêmio

$\begin{array}{lrrrrrrrr}\text { SD 0 } & 2,09 & 2,09 & 0,909 & 0,909 & 0 & 64,60 \% & 20,02 \% & 15,38 \% \\ \text { SD I } & 2,09 & 1,642 & 0,909 & 0,461 & 0,448 & 66,68 \% & 23,36 \% & 9,96 \% \\ \text { "Chance" I } & 2,066 & 2,066 & 0,93 & 0,93 & 0 & 63,61 \% & 20,33 \% & 16,06 \% \\ \text { "Chance" II } & 2,473 & 1,982 & 1,373 & 0,882 & 0,491 & 63,04 \% & 20,98 \% & 15,97 \% \\ \text { Implícito I } & 1,4 & 1,4 & 1,5 & 1,5 & 0 & 35,40 \% & 24,74 \% & 39,86 \% \\ \text { Implícito II } & 1,4 & 0,908 & 1,5 & 1,008 & 0,492 & 31,53 \% & 31,61 \% & 36,86 \%\end{array}$

Implícito II $\quad 1,4 \quad 0,908 \quad 1,5 \quad 1,008 \quad 0,492 \quad 31,53 \% \quad 31,61 \% \quad 36,86 \%$

Palmeiras $3 \times 1$ Corinthians

\begin{tabular}{lrrrrrrrr} 
SD 0 & 1,669 & 1,669 & 1,199 & 1,199 & 0 & $48,37 \%$ & $24,17 \%$ & $27,46 \%$ \\
SD I & 1,669 & 1,916 & 1,199 & 1,446 & $-0,247$ & & & \\
"Chance" I & 1,797 & 1,797 & 1,309 & 1,309 & 0 & $48,94 \%$ & $23,08 \%$ & $27,97 \%$ \\
"Chance" II & 2,313 & 1,822 & 1,848 & 1,357 & 0,491 & $48,44 \%$ & $22,87 \%$ & $28,69 \%$ \\
Implícito I & 1,6 & 1,6 & 1,5 & 1,5 & 0 & $40,26 \%$ & $23,82 \%$ & $35,92 \%$ \\
\hline
\end{tabular}

Implícito II $\quad 1,6 \quad 0,908 \quad 1,5 \quad 0,808 \quad 0,692 \quad 35,89 \% \quad 33,80 \% 30,32 \%$

Goiás $3 \times 1$ Portuguesa

\begin{tabular}{|c|c|c|c|c|c|c|c|c|}
\hline SD 0 & 1,312 & 1,312 & 1,558 & 1,558 & 0 & $32,14 \%$ & $24,71 \%$ & $43,15 \%$ \\
\hline SD I & 1,312 & 0,593 & 1,558 & 0,839 & 0,719 & $24,02 \%$ & $37,33 \%$ & $38,65 \%$ \\
\hline "Chance" I & 1,159 & 1,159 & 1,614 & 1,614 & 0 & $27,38 \%$ & $24,68 \%$ & $47,94 \%$ \\
\hline "Chance" II & 1,697 & 1,206 & 2,165 & 1,674 & 0,491 & $27,56 \%$ & $24,13 \%$ & $48,31 \%$ \\
\hline Implícito I & 1,3 & 1,3 & 2 & 2 & 0 & $24,70 \%$ & $21,64 \%$ & $53,67 \%$ \\
\hline Implícito II & 1,3 & 0,526 & 2 & 1,226 & 0,774 & $15,91 \%$ & $30,47 \%$ & $53,62 \%$ \\
\hline
\end{tabular}


Juventude 0x0 Guarani

$\begin{array}{lrrrrrrrr}\text { SD 0 } & 1,53 & 1,53 & 0,944 & 0,944 & 0 & 50,94 \% & 25,76 \% & 23,30 \% \\ \text { SD I } & 1,53 & 0,852 & 0,944 & 0,266 & 0,678 & 48,38 \% & 40,53 \% & 11,08 \% \\ \text { "Chance" I } & 1,49 & 1,49 & 0,983 & 0,983 & 0 & 48,95 \% & 26,12 \% & 24,93 \% \\ \text { "Chance" II } & 2,045 & 1,554 & 1,468 & 0,977 & 0,491 & 50,74 \% & 25,47 \% & 23,79 \% \\ \text { Implícito I } & 1,5 & 1,5 & 1,4 & 1,4 & 0 & 39,86 \% & 24,74 \% & 35,40 \% \\ \text { Implícito II } & 1,5 & 1,008 & 1,4 & 0,908 & 0,492 & 36,86 \% & 31,61 \% & 31,53 \%\end{array}$

Internacional 0x1 Atlético PR

$\begin{array}{lrrrrrrrr}\text { SD 0 } & 1,755 & 1,755 & 0,816 & 0,816 & 0 & 59,69 \% & 23,20 \% & 17,11 \% \\ \text { SD I } & 1,755 & 1,551 & 0,816 & 0,611 & 0,204 & 60,19 \% & 25,30 \% & 14,51 \% \\ \text { "Chance" I } & 1,677 & 1,677 & 0,883 & 0,883 & 0 & 56,14 \% & 24,18 \% & 19,67 \% \\ \text { "Chance" II } & 2,147 & 1,656 & 1,321 & 0,83 & 0,491 & 56,98 \% & 24,35 \% & 18,66 \% \\ \text { Implícito I } & 2 & 2 & 1,4 & 1,4 & 0 & 51,48 \% & 21,66 \% & 26,86 \% \\ \text { Implícito II } & 2 & 1,008 & 1,4 & 0,408 & 0,992 & 49,95 \% & 35,32 \% & 14,73 \%\end{array}$

Cruzeiro 4x1 América MG

$\begin{array}{lrrrrrrrr}\text { SD 0 } & 1,554 & 1,554 & 0,831 & 0,831 & 0 & 54,41 \% & 25,54 \% & 20,05 \% \\ \text { SD I } & 1,554 & 1,814 & 0,831 & 1,091 & -0,26 & & & \\ \text { "Chance" I } & 1,667 & 1,667 & 1,011 & 1,011 & 0 & 52,74 \% & 24,34 \% & 22,92 \% \\ \text { "Chance" II } & 2,185 & 1,694 & 1,528 & 1,037 & 0,491 & 52,75 \% & 24,08 \% & 23,17 \% \\ \text { Implícito I } & 2 & 2 & 1,3 & 1,3 & 0 & 53,67 \% & 21,64 \% & 24,70 \% \\ \text { Implícito II } & 2 & 1,108 & 1,3 & 0,408 & 0,892 & 53,36 \% & 33,07 \% & 13,57 \%\end{array}$

Implícito II

$0,892 \quad 53,36 \% \quad 33,07 \% \quad 13,57 \%$

Ponte Preta 2x1 Sport

$\begin{array}{lrrrrrrrr}\text { SD 0 } & 0,84 & 0,84 & 1,374 & 1,374 & 0 & 22,93 \% & 27,68 \% & 49,39 \% \\ \text { SD I } & 0,84 & -0,091 & 1,374 & 0,443 & 0,931 & & & \\ \text { "Chance" I } & 0,801 & 0,801 & 1,371 & 1,371 & 0 & 21,91 \% & 27,80 \% & 50,29 \% \\ \text { "Chance" II } & 1,313 & 0,822 & 1,902 & 1,411 & 0,491 & 21,87 \% & 27,26 \% & 50,87 \% \\ \text { Implícito I } & 1 & 1 & 1,5 & 1,5 & 0 & 25,22 \% & 25,98 \% & 48,79 \% \\ \text { Implícito II } & 1 & 0,803 & 1,5 & 1,303 & 0,197 & 23,00 \% & 28,66 \% & 48,34 \%\end{array}$

Implícito II

$0,197 \quad 23,00 \% \quad 28,66 \% \quad 48,34 \%$

Coritiba 1xy Santos

$\begin{array}{lrrrrrrrr}\text { SD 0 } & 1,004 & 1,004 & 1,471 & 1,471 & 0 & 25,80 \% & 26,26 \% & 47,94 \% \\ \text { SD I } & 1,004 & 0,963 & 1,471 & 1,43 & 0,041 & 25,38 \% & 26,77 \% & 47,85 \% \\ \text { "Chance" I } & 1,064 & 1,064 & 1,297 & 1,297 & 0 & 30,48 \% & 27,69 \% & 41,82 \% \\ \text { "Chance" II } & 1,559 & 1,068 & 1,863 & 1,372 & 0,491 & 29,21 \% & 27,00 \% & 43,79 \% \\ \text { Implícito I } & 1,2 & 1,2 & 2 & 2 & 0 & 22,54 \% & 21,55 \% & 55,91 \% \\ \text { Implícito II } & 1,2 & 0,408 & 2 & 1,208 & 0,792 & 12,50 \% & 30,94 \% & 56,56 \%\end{array}$

Ponte Preta 2x1 Vasco

$\begin{array}{lrrrrrrrr}\text { SD 0 } & 0,799 & 0,799 & 1,411 & 1,411 & 0 & 21,24 \% & 27,29 \% & 51,46 \% \\ \text { SD I } & 0,799 & -0,144 & 1,411 & 0,468 & 0,943 & & & \\ \text { "Chance" I } & 0,793 & 0,793 & 1,39 & 1,39 & 0 & 21,39 \% & 27,56 \% & 51,05 \% \\ \text { "Chance" II } & 1,228 & 0,78 & 1,868 & 1,42 & 0,447 & 20,60 \% & 27,20 \% & 52,20 \% \\ \text { Implícito I } & 1 & 1 & 2 & 2 & 0 & 18,26 \% & 21,17 \% & 60,57 \% \\ \text { Implícito II } & 1 & 0,303 & 2 & 1,303 & 0,697 & 8,51 \% & 28,82 \% & 62,67 \%\end{array}$


Flamengo $3 \times 2$ sport

$\begin{array}{lrrrrrrrr}\text { SD 0 } & 1,405 & 1,405 & 1,431 & 1,431 & 0 & 36,87 \% & 25,09 \% & 38,04 \% \\ \text { SD I } & 1,405 & 1,475 & 1,431 & 1,501 & -0,07 & & & \\ \text { "Chance" I } & 1,368 & 1,368 & 1,373 & 1,373 & 0 & 37,08 \% & 25,59 \% & 37,33 \% \\ \text { "Chance" II } & 1,858 & 1,411 & 1,886 & 1,438 & 0,447 & 36,87 \% & 25,03 \% & 38,11 \% \\ \text { Implícito I } & 1,4 & 1,4 & 1,4 & 1,4 & 0 & 37,36 \% & 25,28 \% & 37,36 \% \\ \text { Implícito II } & 1,4 & 1,008 & 1,4 & 1,008 & 0,392 & 34,65 \% & 30,70 \% & 34,65 \%\end{array}$

Botafogo $2 \times 3$ Coritiba

$\begin{array}{lrrrrrrrr}\text { SD 0 } & 1,642 & 1,642 & 0,679 & 0,679 & 0 & 60,61 \% & 24,22 \% & 15,17 \% \\ \text { SD I } & 1,642 & 1,159 & 0,679 & 0,196 & 0,483 & 61,78 \% & 32,01 \% & 6,21 \% \\ \text { "Chance" I } & 1,447 & 1,447 & 0,849 & 0,849 & 0 & 51,14 \% & 26,79 \% & 22,07 \% \\ \text { "Chance" II } & 1,915 & 1,468 & 1,205 & 0,757 & 0,447 & 54,10 \% & 26,59 \% & 19,31 \% \\ \text { Implícito I } & 1,4 & 1,4 & 1,3 & 1,3 & 0 & 39,41 \% & 25,78 \% & 34,81 \% \\ \text { Implícito II } & 1,4 & 1,003 & 1,3 & 0,903 & 0,397 & 36,81 \% & 31,72 \% & 31,47 \%\end{array}$

Corinthians $2 \times 2$ Goiás

$\begin{array}{lrrrrrrrr}\text { SD 0 } & 1,805 & 1,805 & 0,73 & 0,73 & 0 & 63,05 \% & 22,36 \% & 14,59 \% \\ \text { SD I } & 1,805 & 1,595 & 0,73 & 0,52 & 0,21 & 63,87 \% & 24,35 \% & 11,78 \% \\ \text { "Chance" I } & 1,921 & 1,921 & 0,816 & 0,816 & 0 & 63,39 \% & 21,38 \% & 15,24 \% \\ \text { "Chance" II } & 2,299 & 1,852 & 1,168 & 0,72 & 0,447 & 64,33 \% & 21,77 \% & 13,89 \% \\ \text { Implícito I } & 2 & 2 & 1,3 & 1,3 & 0 & 53,67 \% & 21,64 \% & 24,70 \%\end{array}$

Implícito II

$0,997 \quad 52,99 \% \quad 35,98 \% \quad 11,03 \%$

Bragantino 1x0 Grêmio

$\begin{array}{lrrrrrrrr}\text { SD 0 } & 1,649 & 1,649 & 1,291 & 1,291 & 0 & 45,83 \% & 24,15 \% & 30,02 \% \\ \text { SD I } & 1,649 & 1,926 & 1,291 & 1,568 & -0,277 & & & \\ \text { "Chance" I } & 1,415 & 1,415 & 1,184 & 1,184 & 0 & 42,31 \% & 26,18 \% & 31,51 \% \\ \text { "Chance" II } & 2,026 & 1,579 & 1,714 & 1,267 & 0,447 & 44,65 \% & 24,71 \% & 30,64 \% \\ \text { Implícito I } & 1,4 & 1,4 & 1,7 & 1,7 & 0 & 31,74 \% & 23,57 \% & 44,69 \% \\ \text { Implícito II } & 1,4 & 0,603 & 1,7 & 0,903 & 0,797 & 23,25 \% & 36,02 \% & 40,73 \%\end{array}$

Atlético PR 4x1 Vitória

$\begin{array}{lrrrrrrrr}\text { SD 0 } & 1,72 & 1,72 & 1,385 & 1,385 & 0 & 45,48 \% & 23,48 \% & 31,04 \% \\ \text { SD I } & 1,72 & 2,044 & 1,385 & 1,709 & -0,324 & & & \\ \text { "Chance" I } & 1,846 & 1,846 & 1,212 & 1,212 & 0 & 52,21 \% & 22,78 \% & 25,01 \% \\ \text { "Chance" II } & 2,248 & 1,801 & 1,695 & 1,248 & 0,447 & 50,37 \% & 23,11 \% & 26,52 \% \\ \text { Implícito I } & 1,4 & 1,4 & 1,4 & 1,4 & 0 & 37,36 \% & 25,28 \% & 37,36 \%\end{array}$

Internacional $3 \times 1$ Cruzeiro

$\begin{array}{lrrrrrrrr}\text { SD 0 } & 1,425 & 1,425 & 0,785 & 0,785 & 0 & 52,21 \% & 27,13 \% & 20,66 \% \\ \text { SD I } & 1,425 & 1,235 & 0,785 & 0,595 & 0,19 & 51,93 \% & 30,18 \% & 17,89 \% \\ \text { "Chance" I } & 1,414 & 1,414 & 0,841 & 0,841 & 0 & 50,47 \% & 27,20 \% & 22,34 \% \\ \text { "Chance" II } & 1,887 & 1,44 & 1,229 & 0,782 & 0,447 & 52,69 \% & 26,95 \% & 20,36 \% \\ \text { Implícito I } & 1,7 & 1,7 & 1,5 & 1,5 & 0 & 42,61 \% & 23,31 \% & 34,08 \% \\ \text { Implícito II } & 1,7 & 1,026 & 1,5 & 0,826 & 0,674 & 39,35 \% & 32,09 \% & 28,56 \%\end{array}$


Portuguesa $2 \times 3$ Palmeiras

$\begin{array}{lrrrrrrrr}\text { SD 0 } & 1,459 & 1,459 & 1,901 & 1,901 & 0 & 29,71 \% & 22,25 \% & 48,05 \% \\ \text { SD I } & 1,459 & 1,543 & 1,901 & 1,985 & -0,084 & & & \\ \text { "Chance" I } & 1,537 & 1,537 & 2,04 & 2,04 & 0 & 29,19 \% & 21,36 \% & 49,44 \% \\ \text { "Chance" II } & 2,059 & 1,612 & 2,445 & 1,997 & 0,447 & 31,49 \% & 21,52 \% & 46,98 \% \\ \text { Implícito I } & 1,5 & 1,5 & 2 & 2 & 0 & 29,03 \% & 21,62 \% & 49,36 \% \\ \text { Implícito II } & 1,5 & 0,408 & 2 & 0,908 & 1,092 & 15,99 \% & 37,72 \% & 46,29 \%\end{array}$

São Paulo 3x0 Paraná

$\begin{array}{lrrrrrrrr}\text { SD 0 } & 2,031 & 2,031 & 0,69 & 0,69 & 0 & 68,82 \% & 19,58 \% & 11,60 \% \\ \text { SD I } & 2,031 & 1,488 & 0,69 & 0,147 & 0,543 & 72,57 \% & 24,00 \% & 3,43 \% \\ \text { "Chance" I } & 2,014 & 2,014 & 0,775 & 0,775 & 0 & 66,34 \% & 20,21 \% & 13,45 \% \\ \text { "Chance" II } & 2,367 & 1,919 & 1,111 & 0,664 & 0,447 & 67,23 \% & 20,71 \% & 12,05 \% \\ \text { Implícito I } & 1,3 & 1,3 & 1,2 & 1,2 & 0 & 38,90 \% & 26,96 \% & 34,14 \% \\ \text { Implícito II } & 1,3 & 1,103 & 1,2 & 1,003 & 0,197 & 37,63 \% & 29,86 \% & 32,51 \%\end{array}$

Flamengo $4 \times 1$ Corinthians

\begin{tabular}{|c|c|c|c|c|c|c|c|c|}
\hline $\mathrm{SD} \quad 0$ & 1,53 & 1,53 & 1,466 & 1,466 & 0 & $39,26 \%$ & $24,31 \%$ & $36,44 \%$ \\
\hline SD I & 1,53 & 1,421 & 1,466 & 1,356 & 0,11 & $38,76 \%$ & $25,39 \%$ & $35,85 \%$ \\
\hline "Chance" I & 1,49 & 1,49 & 1,378 & 1,378 & 0 & $40,06 \%$ & $24,89 \%$ & $35,05 \%$ \\
\hline "Chance" II & 2,005 & 1,514 & 1,929 & 1,437 & 0,491 & $39,44 \%$ & $24,51 \%$ & $36,05 \%$ \\
\hline Implícito I & 1,4 & 1,4 & 1,5 & 1,5 & 0 & $35,40 \%$ & $24,74 \%$ & $39,86 \%$ \\
\hline Implícito II & 1,4 & 0,908 & 1,5 & 1,008 & 0,492 & $31,53 \%$ & $31,61 \%$ & $36,86 \%$ \\
\hline
\end{tabular}

Atlético MG $5 \times 5$ Botafogo

$\begin{array}{lrrrrrrrr}\text { SD 0 } & 1,764 & 1,764 & 1,201 & 1,201 & 0 & 50,58 \% & 23,42 \% & 26,00 \% \\ \text { SD I } & 1,764 & 1,211 & 1,201 & 0,648 & 0,553 & 49,73 \% & 30,43 \% & 19,84 \% \\ \text { "Chance" I } & 1,714 & 1,714 & 1,187 & 1,187 & 0 & 49,73 \% & 23,83 \% & 26,45 \% \\ \text { "Chance" II } & 2,199 & 1,708 & 1,697 & 1,205 & 0,491 & 49,16 \% & 23,86 \% & 26,98 \% \\ \text { Implícito I } & 1,8 & 1,8 & 1,5 & 1,5 & 0 & 44,92 \% & 22,77 \% & 32,32 \% \\ \text { Implícito II } & 1,8 & 1,026 & 1,5 & 0,726 & 0,774 & 41,81 \% & 32,88 \% & 25,31 \%\end{array}$

Implícito II $\quad 1,8 \quad 1,026 \quad 1,5 \quad 0,726 \quad 0,774 \quad 41,81 \% \quad 32,88 \% \quad 25,31 \%$

Palmeiras 1x0 Internacional

\begin{tabular}{|c|c|c|c|c|c|c|c|c|}
\hline$S D 0$ & 1,864 & 1,864 & 0,843 & 0,843 & 0 & $61,47 \%$ & $22,07 \%$ & $16,45 \%$ \\
\hline $\mathrm{SD} I$ & 1,864 & 1,715 & 0,843 & 0,694 & 0,149 & $61,94 \%$ & $23,32 \%$ & $14,73 \%$ \\
\hline "Chance" I & 1,758 & 1,758 & 0,891 & 0,891 & 0 & $57,87 \%$ & $23,32 \%$ & $18,81 \%$ \\
\hline "Chance" II & 2,322 & 1,831 & 1,338 & 0,847 & 0,491 & $60,64 \%$ & $22,44 \%$ & $16,92 \%$ \\
\hline Implícito I & 2 & 2 & 1,4 & 1,4 & 0 & $51,48 \%$ & $21,66 \%$ & $26,86 \%$ \\
\hline
\end{tabular}

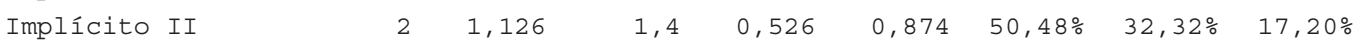

Vasco $3 \times 1$ Santos

$\begin{array}{lrrrrrrrr}\text { SD 0 } & 1,497 & 1,497 & 1,121 & 1,121 & 0 & 45,86 \% & 25,74 \% & 28,40 \% \\ \text { SD I } & 1,497 & 1,688 & 1,121 & 1,312 & -0,191 & & & \\ \text { "Chance" I } & 1,476 & 1,476 & 1,098 & 1,098 & 0 & 45,83 \% & 25,98 \% & 28,19 \% \\ \text { "Chance" II } & 1,995 & 1,504 & 1,662 & 1,17 & 0,491 & 44,91 \% & 25,54 \% & 29,55 \% \\ \text { Implícito I } & 1,6 & 1,6 & 1,6 & 1,6 & 0 & 38,29 \% & 23,43 \% & 38,29 \% \\ \text { Implícito II } & 1,6 & 0,808 & 1,6 & 0,808 & 0,792 & 32,44 \% & 35,12 \% & 32,44 \%\end{array}$


Portuguesa $2 \times 1$ Juventude

$\begin{array}{lrrrrrrrr}\text { SD 0 } & 1,692 & 1,692 & 0,769 & 0,769 & 0 & 59,43 \% & 23,82 \% & 16,75 \% \\ \text { SD I } & 1,692 & 0,281 & 0,769 & -0,642 & 1,411 & & & \\ \text { "Chance" I } & 1,614 & 1,614 & 0,819 & 0,819 & 0 & 56,23 \% & 24,82 \% & 18,95 \% \\ \text { "Chance" II } & 2,168 & 1,677 & 1,236 & 0,744 & 0,491 & 59,71 \% & 23,95 \% & 16,34 \% \\ \text { Implícito I } & 2 & 2 & 1,4 & 1,4 & 0 & 51,48 \% & 21,66 \% & 26,86 \% \\ \text { Implícito II } & 2 & 1,008 & 1,4 & 0,408 & 0,992 & 49,95 \% & 35,32 \% & 14,73 \%\end{array}$

Atlético PR 2xo América RN

$\begin{array}{lrrrrrrrr}\text { SD 0 } & 2,135 & 2,135 & 0,854 & 0,854 & 0 & 66,81 \% & 19,35 \% & 13,84 \% \\ \text { SD I } & 2,135 & 2,275 & 0,854 & 0,994 & -0,14 & & & \\ \text { "Chance" I } & 2,255 & 2,255 & 0,874 & 0,874 & 0 & 68,59 \% & 18,31 \% & 13,10 \% \\ \text { "Chance" II } & 2,638 & 2,146 & 1,279 & 0,787 & 0,491 & 68,62 \% & 18,90 \% & 12,47 \% \\ \text { Implícito I } & 2 & 2 & 1,2 & 1,2 & 0 & 55,91 \% & 21,55 \% & 22,54 \% \\ \text { Implícito II } & 2 & 1,326 & 1,2 & 0,526 & 0,674 & 56,57 \% & 28,70 \% & 14,72 \%\end{array}$

Ponte Preta 4x2 Vitória

$\begin{array}{lrrrrrrrr}\text { SD 0 } & 1,392 & 1,392 & 1,542 & 1,542 & 0 & 34,40 \% & 24,53 \% & 41,06 \% \\ \text { SD I } & 1,392 & 0,835 & 1,542 & 0,985 & 0,557 & 29,66 \% & 32,53 \% & 37,81 \% \\ \text { "Chance" I } & 1,373 & 1,373 & 1,411 & 1,411 & 0 & 36,46 \% & 25,36 \% & 38,18 \% \\ \text { "Chance" II } & 1,972 & 1,481 & 1,95 & 1,458 & 0,491 & 38,21 \% & 24,58 \% & 37,20 \% \\ \text { Implícito I } & 1,4 & 1,4 & 1,4 & 1,4 & 0 & 37,36 \% & 25,28 \% & 37,36 \% \\ \text { Implícito II } & 1,4 & 0,903 & 1,4 & 0,903 & 0,497 & 33,59 \% & 32,83 \% & 33,59 \%\end{array}$

São Paulo 3x1 Goiás

$\begin{array}{lrrrrrrrr}\text { SD 0 } & 1,937 & 1,937 & 0,805 & 0,805 & 0 & 64,01 \% & 21,16 \% & 14,83 \% \\ \text { SD I } & 1,937 & 1,407 & 0,805 & 0,275 & 0,53 & 66,43 \% & 26,52 \% & 7,05 \% \\ \text { "Chance" I } & 2,079 & 2,079 & 0,868 & 0,868 & 0 & 65,37 \% & 19,96 \% & 14,67 \% \\ \text { "Chance" II } & 2,466 & 1,974 & 1,286 & 0,794 & 0,491 & 65,06 \% & 20,71 \% & 14,22 \% \\ \text { Implícito I } & 1,4 & 1,4 & 1,4 & 1,4 & 0 & 37,36 \% & 25,28 \% & 37,36 \% \\ \text { Implícito II } & 1,4 & 0,903 & 1,4 & 0,903 & 0,497 & 33,59 \% & 32,83 \% & 33,59 \%\end{array}$

Implícito II $\quad 1,4 \quad 0,903 \quad 1,4 \quad 0,903 \quad 0,497 \quad 33,59 \% \quad 32,83 \% \quad 33,59 \%$

Paraná 1x2 Cruzeiro

$\begin{array}{lrrrrrrrr}\text { SD 0 } & 1,111 & 1,111 & 1,237 & 1,237 & 0 & 32,94 \% & 27,95 \% & 39,11 \% \\ \text { SD I } & 1,111 & 1,143 & 1,237 & 1,269 & -0,032 & & & \\ \text { "Chance" I } & 1,066 & 1,066 & 1,134 & 1,134 & 0 & 33,75 \% & 29,11 \% & 37,14 \% \\ \text { "Chance" II } & 1,585 & 1,093 & 1,655 & 1,163 & 0,491 & 33,92 \% & 28,67 \% & 37,41 \% \\ \text { Implícito I } & 1,2 & 1,2 & 1,5 & 1,5 & 0 & 30,37 \% & 25,48 \% & 44,15 \%\end{array}$

Implícito II $\quad 1,2 \quad 0,803 \quad 1,5 \quad 1,103 \quad 0,397 \quad 26,39 \% \quad 31,26 \%$

Grêmio $1 \times 1$ Coritiba

\begin{tabular}{|c|c|c|c|c|c|c|c|c|}
\hline SD 0 & 1,409 & 1,409 & 1,16 & 1,16 & 0 & $42,68 \%$ & $26,33 \%$ & $30,99 \%$ \\
\hline SD I & 1,409 & 1,055 & 1,16 & 0,806 & 0,354 & $40,76 \%$ & $31,88 \%$ & $27,37 \%$ \\
\hline "Chance" I & 1,179 & 1,179 & 1,133 & 1,133 & 0 & $36,99 \%$ & $28,27 \%$ & $34,73 \%$ \\
\hline "Chance" II & 1,699 & 1,207 & 1,665 & 1,174 & 0,491 & $36,92 \%$ & $27,79 \%$ & $35,29 \%$ \\
\hline Implícito I & 1,4 & 1,4 & 1,4 & 1,4 & 0 & $37,36 \%$ & $25,28 \%$ & $37,36 \%$ \\
\hline Implícito II & 1,4 & 0,903 & 1,4 & 0,903 & 0,497 & $33,59 \%$ & $32,83 \%$ & $33,59 \%$ \\
\hline
\end{tabular}


Sport 3×2 América MG

$\begin{array}{lrrrrrrrr}\text { SD 0 } & 2,022 & 2,022 & 0,541 & 0,541 & 0 & 72,45 \% & 18,74 \% & 8,81 \% \\ \text { SD I } & 2,022 & 2,055 & 0,541 & 0,574 & -0,033 & & & \\ \text { "Chance" I } & 2,089 & 2,089 & 0,692 & 0,692 & 0 & 69,89 \% & 18,96 \% & 11,14 \% \\ \text { "Chance" II } & 2,5 & 2,009 & 1,037 & 0,545 & 0,491 & 72,08 \% & 18,93 \% & 8,98 \% \\ \text { Implícito I } & 2 & 2 & 1,2 & 1,2 & 0 & 55,91 \% & 21,55 \% & 22,54 \% \\ \text { Implícito II } & 2 & 1,208 & 1,2 & 0,408 & 0,792 & 56,56 \% & 30,94 \% & 12,50 \%\end{array}$

Atlético MG 2x0 Internacional

\begin{tabular}{|c|c|c|c|c|c|c|c|c|}
\hline $\mathrm{SD} 0$ & 1,555 & 1,555 & 1,292 & 1,292 & 0 & $43,50 \%$ & $24,80 \%$ & $31,70 \%$ \\
\hline SD I & 1,555 & 0,486 & 1,292 & 0,223 & 1,069 & $32,36 \%$ & $54,69 \%$ & $12,95 \%$ \\
\hline "Chance" I & 1,424 & 1,424 & 1,264 & 1,264 & 0 & $40,79 \%$ & $25,79 \%$ & $33,41 \%$ \\
\hline "Chance" II & 1,982 & 1,504 & 1,777 & 1,299 & 0,478 & $42,07 \%$ & $25,12 \%$ & $32,82 \%$ \\
\hline Implícito I & 2,4 & 2,4 & 2 & 2 & 0 & $47,67 \%$ & $19,34 \%$ & $32,98 \%$ \\
\hline Implícito II & 2,4 & 0,659 & 2 & 0,259 & 1,741 & $40,16 \%$ & $47,03 \%$ & $12,81 \%$ \\
\hline
\end{tabular}

Implícito II

$2,4 \quad 0,659 \quad 2 \quad 0,259$

$1,74140,16 \% \quad 47,03 \% \quad 12,81 \%$

Bragantino 1x2 Guarani

$\begin{array}{lrrrrrrrr}\text { SD 0 } & 1,317 & 1,317 & 1,408 & 1,408 & 0 & 35,09 \% & 25,65 \% & 39,26 \% \\ \text { SD I } & 1,317 & 1,642 & 1,408 & 1,733 & -0,325 & & \\ \text { "Chance" I } & 1,21 & 1,21 & 1,356 & 1,356 & 0 & 33,32 \% & 26,51 \% & 40,17 \% \\ \text { "Chance" II } & 1,829 & 1,351 & 1,912 & 1,434 & 0,478 & 35,45 \% & 25,33 \% & 39,22 \% \\ \text { Implícito I } & 1,3 & 1,3 & 2 & 2 & 0 & 24,70 \% & 21,64 \% & 53,67 \% \\ \text { Implícito II } & 1,3 & 0,408 & 2 & 1,108 & 0,892 & 13,57 \% & 33,07 \% & 53,36 \%\end{array}$

Implícito II

$1,3 \quad 0,408 \quad 2 \quad 1,108$

$0,892 \quad 13,57 \% \quad 33,07 \% \quad 53,36 \%$

Juventude 1x2 Coritiba

$\begin{array}{lrrrrrrrr}\text { SD 0 } & 1,282 & 1,282 & 0,466 & 0,466 & 0 & 57,05 \% & 29,48 \% & 13,47 \% \\ \text { SD I } & 1,282 & 0,685 & 0,466 & -0,13 & 0,596 & & & \\ \text { "Chance" I } & 1,096 & 1,096 & 0,724 & 0,724 & 0 & 44,13 \% & 31,84 \% & 24,03 \% \\ \text { "Chance" II } & 1,606 & 1,128 & 1,028 & 0,55 & 0,478 & 49,88 \% & 32,18 \% & 17,94 \% \\ \text { Implícito I } & 1,4 & 1,4 & 1,4 & 1,4 & 0 & 37,36 \% & 25,28 \% & 37,36 \% \\ \text { Implícito II } & 1,4 & 1,008 & 1,4 & 1,008 & 0,392 & 34,65 \% & 30,70 \% & 34,65 \%\end{array}$

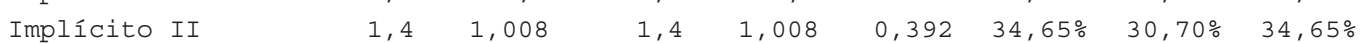

Santos $3 \times 3$ América MG

$\begin{array}{lrrrrrrrr}\text { SD 0 } & 2,324 & 2,324 & 0,522 & 0,522 & 0 & 78,04 \% & 15,30 \% & 6,66 \% \\ \text { SD I } & 2,324 & 2,592 & 0,522 & 0,79 & -0,268 & & & \\ \text { "Chance" I } & 2,519 & 2,519 & 0,706 & 0,706 & 0 & 76,80 \% & 14,88 \% & 8,31 \% \\ \text { "Chance" II } & 2,787 & 2,309 & 1,028 & 0,55 & 0,478 & 77,12 \% & 15,68 \% & 7,18 \% \\ \text { Implícito I } & 2,3 & 2,3 & 1 & 1 & 0 & 66,51 \% & 18,49 \% & 14,99 \% \\ \text { Implícito II } & 2,3 & 1,526 & 1 & 0,226 & 0,774 & 70,97 \% & 23,86 \% & 5,16 \%\end{array}$

Internacional 1xo Vasco

\begin{tabular}{|c|c|c|c|c|c|c|c|c|}
\hline SD 0 & 1,305 & 1,305 & 0,945 & 0,945 & 0 & $44,85 \%$ & $28,14 \%$ & $27,01 \%$ \\
\hline SD I & 1,305 & 1,259 & 0,945 & 0,899 & 0,046 & $44,66 \%$ & $28,84 \%$ & $26,50 \%$ \\
\hline "Chance" I & 1,219 & 1,219 & 0,976 & 0,976 & 0 & $41,65 \%$ & $28,89 \%$ & $29,46 \%$ \\
\hline "Chance" II & 1,686 & 1,269 & 1,385 & 0,969 & 0,416 & $43,27 \%$ & $28,41 \%$ & $28,32 \%$ \\
\hline Implícito I & 1,8 & 1,8 & 1,5 & 1,5 & 0 & $44,92 \%$ & $22,77 \%$ & $32,32 \%$ \\
\hline Implícito II & 1,8 & 0,908 & 1,5 & 0,608 & 0,892 & $40,79 \%$ & $35,86 \%$ & $23,35 \%$ \\
\hline
\end{tabular}


Botafogo $3 \times 1$ Palmeiras

$\begin{array}{lrrrrrrrr}\text { SD 0 } & 1,429 & 1,429 & 1,625 & 1,625 & 0 & 33,76 \% & 23,93 \% & 42,30 \% \\ \text { SD I } & 1,429 & 0,692 & 1,625 & 0,889 & 0,737 & 26,72 \% & 35,32 \% & 37,96 \% \\ \text { "Chance" I } & 1,316 & 1,316 & 1,537 & 1,537 & 0 & 32,61 \% & 24,84 \% & 42,54 \% \\ \text { "Chance" II } & 1,789 & 1,373 & 2,038 & 1,622 & 0,416 & 32,47 \% & 24,12 \% & 43,41 \% \\ \text { Implícito I } & 1,4 & 1,4 & 2,2 & 2,2 & 0 & 23,99 \% & 20,33 \% & 55,68 \% \\ \text { Implícito II } & 1,4 & 0,459 & 2,2 & 1,259 & 0,941 & 13,52 \% & 29,90 \% & 56,58 \%\end{array}$

Vitória 1x4 Flamengo

$\begin{array}{lrrrrrrrr}\text { SD 0 } & 2,204 & 2,204 & 1,393 & 1,393 & 0 & 55,91 \% & 20,30 \% & 23,79 \% \\ \text { SD I } & 2,204 & 2,759 & 1,393 & 1,948 & -0,555 & & & \\ \text { "Chance" I } & 2,136 & 2,136 & 1,496 & 1,496 & 0 & 52,33 \% & 20,80 \% & 26,87 \% \\ \text { "Chance" II } & 2,467 & 2,051 & 1,966 & 1,55 & 0,416 & 49,41 \% & 21,29 \% & 29,30 \% \\ \text { Implícito I } & 1,7 & 1,7 & 2 & 2 & 0 & 33,33 \% & 21,38 \% & 45,29 \% \\ \text { Implícito II } & 1,7 & 0,526 & 2 & 0,826 & 1,174 & 21,71 \% & 38,40 \% & 39,89 \%\end{array}$

Coritiba $2 \times 1$ são Paulo

$\begin{array}{lrrrrrrrr}\text { SD 0 } & 1,244 & 1,244 & 1,283 & 1,283 & 0 & 35,66 \% & 26,83 \% & 37,51 \% \\ \text { SD I } & 1,244 & 0,679 & 1,283 & 0,718 & 0,565 & 29,65 \% & 38,34 \% & 32,00 \% \\ \text { "Chance" I } & 1,219 & 1,219 & 1,124 & 1,124 & 0 & 38,30 \% & 28,02 \% & 33,68 \% \\ \text { "Chance" II } & 1,653 & 1,237 & 1,554 & 1,137 & 0,416 & 38,52 \% & 27,80 \% & 33,68 \% \\ \text { Implícito I } & 2 & 2 & 1,9 & 1,9 & 0 & 41,47 \% & 20,97 \% & 37,57 \% \\ \text { Implícito II } & 2 & 0,626 & 1,9 & 0,526 & 1,374 & 31,76 \% & 42,91 \% & 25,33 \%\end{array}$

Corinthians 4x2 Atlético PR

$\begin{array}{lrrrrrrrr}\text { SD 0 } & 1,663 & 1,663 & 1,034 & 1,034 & 0 & 52,08 \% & 24,38 \% & 23,55 \% \\ \text { SD I } & 1,663 & 1,535 & 1,034 & 0,906 & 0,128 & 52,01 \% & 25,74 \% & 22,24 \% \\ \text { "Chance" I } & 1,546 & 1,546 & 1,03 & 1,03 & 0 & 49,25 \% & 25,49 \% & 25,26 \% \\ \text { "Chance" II } & 1,968 & 1,552 & 1,428 & 1,012 & 0,416 & 49,84 \% & 25,46 \% & 24,70 \% \\ \text { Implícito I } & 1,9 & 1,9 & 1,7 & 1,7 & 0 & 43,13 \% & 21,83 \% & 35,04 \% \\ \text { Implícito II } & 1,9 & 0,708 & 1,7 & 0,508 & 1,192 & 35,66 \% & 41,30 \% & 23,04 \%\end{array}$

Implícito II $\quad 1,9 \quad 0,708 \quad 1,7 \quad 0,508 \quad 1,192 \quad 35,66 \% \quad 41,30 \% \quad 23,04 \%$

Santos 1x1 Portuguesa

$\begin{array}{lrrrrrrrr}\text { SD 0 } & 2,016 & 2,016 & 1,256 & 1,256 & 0 & 54,99 \% & 21,49 \% & 23,52 \% \\ \text { SD I } & 2,016 & 1,943 & 1,256 & 1,183 & 0,073 & 55,06 \% & 21,99 \% & 22,95 \% \\ \text { "Chance" I } & 2 & 2 & 1,246 & 1,246 & 0 & 54,87 \% & 21,60 \% & 23,53 \% \\ \text { "Chance" II } & 2,375 & 1,959 & 1,723 & 1,307 & 0,416 & 52,62 \% & 21,93 \% & 25,44 \% \\ \text { Implícito I } & 2 & 2 & 2 & 2 & 0 & 39,65 \% & 20,70 \% & 39,65 \% \\ \text { Implícito II } & 2 & 0,813 & 2 & 0,813 & 1,187 & 32,51 \% & 34,98 \% & 32,51 \%\end{array}$

América MG 2xo Bragantino

$\begin{array}{lrrrrrrrr}\text { SD 0 } & 1,696 & 1,696 & 0,992 & 0,992 & 0 & 53,89 \% & 24,06 \% & 22,05 \% \\ \text { SD I } & 1,696 & 1,902 & 0,992 & 1,198 & -0,206 & & & \\ \text { "Chance" I } & 1,56 & 1,56 & 0,917 & 0,917 & 0 & 52,38 \% & 25,46 \% & 22,17 \% \\ \text { "Chance" II } & 2,06 & 1,644 & 1,434 & 1,018 & 0,416 & 52,00 \% & 24,56 \% & 23,44 \% \\ \text { Implícito I } & 1,9 & 1,9 & 1,8 & 1,8 & 0 & 41,21 \% & 21,58 \% & 37,21 \% \\ \text { Implícito II } & 1,9 & 0,503 & 1,8 & 0,403 & 1,397 & 28,94 \% & 49,05 \% & 22,00 \%\end{array}$


Guarani 2x0 Paraná

$\begin{array}{lrrrrrrrr}\text { SD 0 } & 1,929 & 1,929 & 0,854 & 0,854 & 0 & 62,61 \% & 21,42 \% & 15,96 \% \\ \text { SD I } & 1,929 & 2,056 & 0,854 & 0,98 & -0,127 & & & \\ \text { "Chance" I } & 1,858 & 1,858 & 0,901 & 0,901 & 0 & 59,89 \% & 22,30 \% & 17,80 \% \\ \text { "Chance" II } & 2,247 & 1,831 & 1,294 & 0,877 & 0,416 & 59,87 \% & 22,52 \% & 17,60 \% \\ \text { Implícito I } & 2,2 & 2,2 & 1,2 & 1,2 & 0 & 60,06 \% & 19,98 \% & 19,95 \% \\ \text { Implícito II } & 2,2 & 1,326 & 1,2 & 0,326 & 0,874 & 62,60 \% & 28,40 \% & 9,00 \%\end{array}$

Goiás 0x1 Ponte Preta

$\begin{array}{lrrrrrrrr}\text { SD 0 } & 1,653 & 1,653 & 0,89 & 0,89 & 0 & 55,38 \% & 24,45 \% & 20,17 \% \\ \text { SD I } & 1,653 & 0,686 & 0,89 & -0,077 & 0,967 & & & \\ \text { "Chance" I } & 1,593 & 1,593 & 1,001 & 1,001 & 0 & 51,13 \% & 25,07 \% & 23,80 \% \\ \text { "Chance" II } & 2,028 & 1,611 & 1,42 & 1,004 & 0,416 & 51,53 \% & 24,89 \% & 23,59 \% \\ \text { Implícito I } & 2 & 2 & 1,3 & 1,3 & 0 & 53,67 \% & 21,64 \% & 24,70 \% \\ \text { Implícito II } & 2 & 1,108 & 1,3 & 0,408 & 0,892 & 53,36 \% & 33,07 \% & 13,57 \%\end{array}$

Cruzeiro 0x2 Grêmio

$\begin{array}{lrrrrrrrr}\text { SD 0 } & 1,953 & 1,953 & 0,621 & 0,621 & 0 & 69,03 \% & 20,08 \% & 10,89 \% \\ \text { SD I } & 1,953 & 1,936 & 0,621 & 0,604 & 0,017 & 69,14 \% & 20,18 \% & 10,68 \% \\ \text { "Chance" I } & 1,847 & 1,847 & 0,783 & 0,783 & 0 & 62,62 \% & 22,06 \% & 15,32 \% \\ \text { "Chance" II } & 2,252 & 1,836 & 1,065 & 0,648 & 0,416 & 65,87 \% & 21,64 \% & 12,49 \% \\ \text { Implícito I } & 2 & 2 & 1,4 & 1,4 & 0 & 51,48 \% & 21,66 \% & 26,86 \% \\ \text { Implícito II } & 2 & 1,126 & 1,4 & 0,526 & 0,874 & 50,48 \% & 32,32 \% & 17,20 \%\end{array}$

Implícito II

$0,874 \quad 50,48 \% \quad 32,32 \% \quad 17,20 \%$

América RN 1x3 Atlético MG

\begin{tabular}{|c|c|c|c|c|c|c|c|c|}
\hline SD 0 & 1,53 & 1,53 & 1,727 & 1,727 & 0 & $34,29 \%$ & $23,08 \%$ & $42,63 \%$ \\
\hline $\mathrm{SD} I$ & 1,53 & 1,043 & 1,727 & 1,24 & 0,487 & $30,97 \%$ & $28,31 \%$ & $40,72 \%$ \\
\hline "Chance" I & 1,417 & 1,417 & 1,681 & 1,681 & 0 & $32,47 \%$ & $23,64 \%$ & $43,89 \%$ \\
\hline "Chance" II & 1,882 & 1,466 & 2,187 & 1,77 & 0,416 & $32,05 \%$ & $23,00 \%$ & $44,94 \%$ \\
\hline Implícito I & 1,2 & 1,2 & 1,9 & 1,9 & 0 & $23,94 \%$ & $22,35 \%$ & $53,72 \%$ \\
\hline Implícito II & 1,2 & 0,508 & 1,9 & 1,208 & 0,692 & $15,59 \%$ & $30,82 \%$ & $53,59 \%$ \\
\hline Juventude $1 \times 2$ & Sport & & & & & & & \\
\hline SD 0 & 1,154 & 1,154 & 0,929 & 0,929 & 0 & $40,86 \%$ & $29,84 \%$ & $29,30 \%$ \\
\hline SD I & 1,154 & 0,701 & 0,929 & 0,476 & 0,453 & $36,17 \%$ & $41,99 \%$ & $21,83 \%$ \\
\hline "Chance" I & 1,102 & 1,102 & 0,995 & 0,995 & 0 & $37,77 \%$ & $29,93 \%$ & $32,31 \%$ \\
\hline "Chance" II & 1,568 & 1,152 & 1,403 & 0,987 & 0,416 & $39,46 \%$ & $29,50 \%$ & $31,05 \%$ \\
\hline Implícito I & 1,4 & 1,4 & 1,4 & 1,4 & 0 & $37,36 \%$ & $25,28 \%$ & $37,36 \%$ \\
\hline
\end{tabular}

Implícito II

$1,4 \quad 1,126 \quad 1,4 \quad 1,126$

$0,274 \quad 35,63 \% \quad 28,73 \% \quad 35,63 \%$

América RN 2x1 Flamengo

$\begin{array}{lrrrrrrrr}\text { SD 0 } & 1,621 & 1,621 & 1,726 & 1,726 & 0 & 36,40 \% & 22,81 \% & 40,79 \% \\ \text { SD I } & 1,621 & 1,972 & 1,726 & 2,077 & -0,351 & & & \\ \text { "Chance" I } & 1,463 & 1,463 & 1,737 & 1,737 & 0 & 32,56 \% & 23,20 \% & 44,24 \% \\ \text { "Chance" II } & 1,971 & 1,532 & 2,257 & 1,819 & 0,438 & 32,72 \% & 22,59 \% & 44,68 \% \\ \text { Implícito I } & 1 & 1 & 2,4 & 2,4 & 0 & 14,03 \% & 17,65 \% & 68,32 \% \\ \text { Implícito II } & 1 & 0,259 & 2,4 & 1,659 & 0,741 & 5,30 \% & 21,71 \% & 72,98 \%\end{array}$


Paraná 0x0 Corinthians

$\begin{array}{lrrrrrrrr}\text { SD 0 } & 1,231 & 1,231 & 1,457 & 1,457 & 0 & 31,95 \% & 25,69 \% & 42,36 \% \\ \text { SD I } & 1,231 & 1,182 & 1,457 & 1,408 & 0,049 & 31,58 \% & 26,25 \% & 42,17 \% \\ \text { "Chance" I } & 1,09 & 1,09 & 1,346 & 1,346 & 0 & 30,28 \% & 27,15 \% & 42,58 \% \\ \text { "Chance" II } & 1,578 & 1,14 & 1,826 & 1,388 & 0,438 & 30,84 \% & 26,58 \% & 42,58 \% \\ \text { Implícito I } & 1,2 & 1,2 & 2,1 & 2,1 & 0 & 21,21 \% & 20,76 \% & 58,03 \% \\ \text { Implícito II } & 1,2 & 0,426 & 2,1 & 1,326 & 0,774 & 11,85 \% & 28,62 \% & 59,53 \%\end{array}$

Atlético PR $1 \times 2$ Vasco

\begin{tabular}{|c|c|c|c|c|c|c|c|c|}
\hline $\mathrm{SD} 0$ & 1,352 & 1,352 & 1,21 & 1,21 & 0 & $40,08 \%$ & $26,54 \%$ & $33,39 \%$ \\
\hline $\mathrm{SD} I$ & 1,352 & 1,222 & 1,21 & 1,08 & 0,13 & $39,37 \%$ & $28,26 \%$ & $32,37 \%$ \\
\hline "Chance" I & 1,24 & 1,24 & 1,165 & 1,165 & 0 & $38,02 \%$ & $27,61 \%$ & $34,38 \%$ \\
\hline "Chance" II & 1,732 & 1,293 & 1,6 & 1,162 & 0,438 & $39,54 \%$ & $27,22 \%$ & $33,24 \%$ \\
\hline Implícito I & 2 & 2 & 1,9 & 1,9 & 0 & $41,47 \%$ & $20,97 \%$ & $37,57 \%$ \\
\hline Implícito II & 2 & 0,626 & 1,9 & 0,526 & 1,374 & $31,76 \%$ & $42,91 \%$ & $25,33 \%$ \\
\hline
\end{tabular}

Botafogo 1x0 Ponte Preta

$\begin{array}{lrrrrrrrr}\text { SD 0 } & 1,914 & 1,914 & 0,879 & 0,879 & 0 & 61,67 \% & 21,66 \% & 16,67 \% \\ \text { SD I } & 1,914 & 0,149 & 0,879 & -0,886 & 1,765 & & & \\ \text { "Chance" I } & 1,95 & 1,95 & 0,92 & 0,92 & 0 & 61,42 \% & 21,43 \% & 17,15 \% \\ \text { "Chance" II } & 2,306 & 1,868 & 1,322 & 0,884 & 0,438 & 60,53 \% & 22,15 \% & 17,31 \% \\ \text { Implícito I } & 1,5 & 1,5 & 1,2 & 1,2 & 0 & 44,15 \% & 25,48 \% & 30,37 \% \\ \text { Implícito II } & 1,5 & 1,208 & 1,2 & 0,908 & 0,292 & 42,96 \% & 29,37 \% & 27,67 \%\end{array}$

Implícito II $\quad 1,5 \quad 1,208 \quad 1,2 \quad 0,908 \quad 0,292 \quad 42,96 \% \quad 29,37 \% \quad 27,67 \%$

Palmeiras 1x2 Coritiba

$\begin{array}{lrrrrrrrr}\text { SD 0 } & 2,029 & 2,029 & 0,507 & 0,507 & 0 & 73,45 \% & 18,41 \% & 8,13 \% \\ \text { SD I } & 2,029 & 2,024 & 0,507 & 0,502 & 0,005 & 73,49 \% & 18,44 \% & 8,07 \% \\ \text { "Chance" I } & 1,84 & 1,84 & 0,728 & 0,728 & 0 & 63,88 \% & 21,94 \% & 14,18 \% \\ \text { "Chance" II } & 2,286 & 1,848 & 0,991 & 0,552 & 0,438 & 68,69 \% & 20,97 \% & 10,33 \% \\ \text { Implícito I } & 2 & 2 & 1,5 & 1,5 & 0 & 49,36 \% & 21,62 \% & 29,03 \% \\ \text { Implícito II } & 2 & 1,026 & 1,5 & 0,526 & 0,974 & 47,15 \% & 34,26 \% & 18,59 \%\end{array}$

Implícito II

$0,974 \quad 47,15 \% \quad 34,26 \% \quad 18,59 \%$

São Paulo 1×2 Grêmio

$\begin{array}{lrrrrrrrr}\text { SD 0 } & 2,176 & 2,176 & 0,801 & 0,801 & 0 & 68,86 \% & 18,68 \% & 12,46 \% \\ \text { SD I } & 2,176 & 1,809 & 0,801 & 0,434 & 0,367 & 71,12 \% & 20,74 \% & 8,14 \% \\ \text { "Chance" I } & 2,202 & 2,202 & 0,849 & 0,849 & 0 & 68,21 \% & 18,67 \% & 13,11 \% \\ \text { "Chance" II } & 2,505 & 2,067 & 1,198 & 0,76 & 0,438 & 67,77 \% & 19,58 \% & 12,65 \% \\ \text { Implícito I } & 1,5 & 1,5 & 1,4 & 1,4 & 0 & 39,86 \% & 24,74 \% & 35,40 \% \\ \text { Implícito II } & 1,5 & 1,008 & 1,4 & 0,908 & 0,492 & 36,86 \% & 31,61 \% & 31,53 \%\end{array}$

Implícito II $1,5 \quad 1,008 \quad 1,4 \quad 0,908 \quad 0,492 \quad 36,86 \% \quad 31,61 \% 31,53 \%$

Guarani 1x1 Portuguesa

\begin{tabular}{|c|c|c|c|c|c|c|c|c|}
\hline$S D 0$ & 1,682 & 1,682 & 1,5 & 1,5 & 0 & $42,19 \%$ & $23,40 \%$ & $34,41 \%$ \\
\hline SD I & 1,682 & 1,453 & 1,5 & 1,271 & 0,229 & $41,39 \%$ & $25,56 \%$ & $33,05 \%$ \\
\hline "Chance" I & 1,571 & 1,571 & 1,493 & 1,493 & 0 & $39,70 \%$ & $23,99 \%$ & $36,31 \%$ \\
\hline "Chance" II & 2,035 & 1,597 & 1,994 & 1,556 & 0,438 & $39,07 \%$ & $23,62 \%$ & $37,31 \%$ \\
\hline Implícito I & 1,5 & 1,5 & 1,6 & 1,6 & 0 & $35,92 \%$ & $23,82 \%$ & $40,26 \%$ \\
\hline Implícito II & 1,5 & 0,808 & 1,6 & 0,908 & 0,692 & $30,32 \%$ & $33,80 \%$ & $35,89 \%$ \\
\hline
\end{tabular}


Bragantino 0x4 Cruzeiro

$\begin{array}{lrrrrrrrr}\text { SD 0 } & 0,967 & 0,967 & 1,29 & 1,29 & 0 & 27,89 \% & 28,20 \% & 43,90 \% \\ \text { SD I } & 0,967 & 1,456 & 1,29 & 1,779 & -0,489 & & & \\ \text { "Chance" I } & 0,939 & 0,939 & 1,219 & 1,219 & 0 & 28,40 \% & 29,09 \% & 42,51 \% \\ \text { "Chance" II } & 1,434 & 0,996 & 1,724 & 1,286 & 0,438 & 28,77 \% & 28,11 \% & 43,12 \% \\ \text { Implícito I } & 1,3 & 1,3 & 1,8 & 1,8 & 0 & 27,73 \% & 23,07 \% & 49,19 \% \\ \text { Implícito II } & 1,3 & 0,608 & 1,8 & 1,108 & 0,692 & 20,11 \% & 32,29 \% & 47,60 \%\end{array}$

Atlético MG $2 \times 1$ Goiás

$\begin{array}{lrrrrrrrr}\text { SD 0 } & 1,969 & 1,969 & 0,984 & 0,984 & 0 & 60,29 \% & 21,42 \% & 18,29 \% \\ \text { SD I } & 1,969 & 1,221 & 0,984 & 0,236 & 0,748 & 62,39 \% & 30,50 \% & 7,10 \% \\ \text { "Chance" I } & 2,094 & 2,094 & 0,994 & 0,994 & 0 & 62,66 \% & 20,29 \% & 17,04 \% \\ \text { "Chance" II } & 2,441 & 2,002 & 1,422 & 0,984 & 0,438 & 61,01 \% & 21,10 \% & 17,88 \% \\ \text { Implícito I } & 2 & 2 & 1,4 & 1,4 & 0 & 51,48 \% & 21,66 \% & 26,86 \% \\ \text { Implícito II } & 2 & 1,126 & 1,4 & 0,526 & 0,874 & 50,48 \% & 32,32 \% & 17,20 \%\end{array}$

Internacional 1x1 sport

$\begin{array}{lrrrrrrrr}\text { SD 0 } & 1,48 & 1,48 & 0,856 & 0,856 & 0 & 51,85 \% & 26,40 \% & 21,76 \% \\ \text { SD I } & 1,48 & 1,438 & 0,856 & 0,814 & 0,042 & 51,81 \% & 26,94 \% & 21,25 \% \\ \text { "Chance" I } & 1,42 & 1,42 & 0,923 & 0,923 & 0 & 48,56 \% & 26,97 \% & 24,47 \% \\ \text { "Chance" II } & 1,896 & 1,458 & 1,344 & 0,906 & 0,438 & 49,99 \% & 26,58 \% & 23,42 \% \\ \text { Implícito I } & 1,4 & 1,4 & 1,2 & 1,2 & 0 & 41,56 \% & 26,23 \% & 32,21 \% \\ \text { Implícito II } & 1,4 & 1,208 & 1,2 & 1,008 & 0,192 & 40,60 \% & 28,82 \% & 30,58 \%\end{array}$

Sport 2x1 Botafogo

\begin{tabular}{|c|c|c|c|c|c|c|c|c|}
\hline SD 0 & 1,786 & 1,786 & 1,086 & 1,086 & 0 & $53,77 \%$ & $23,25 \%$ & $22,98 \%$ \\
\hline SD I & 1,786 & 1,133 & 1,086 & 0,433 & 0,653 & $53,43 \%$ & $32,46 \%$ & $14,11 \%$ \\
\hline "Chance" I & 1,793 & 1,793 & 1,088 & 1,088 & 0 & $53,87 \%$ & $23,19 \%$ & $22,93 \%$ \\
\hline "Chance" II & 2,189 & 1,775 & 1,514 & 1,1 & 0,415 & $53,18 \%$ & $23,36 \%$ & $23,46 \%$ \\
\hline Implícito I & 2 & 2 & 1 & 1 & 0 & $60,57 \%$ & $21,17 \%$ & $18,26 \%$ \\
\hline lícito T & 2 & 1408 & 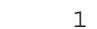 & 0.408 & 0.592 & $6235 \%$ & $2704 \%$ & $10.60 \%$ \\
\hline
\end{tabular}

Implícito II

$0,592 \quad 62,35 \% \quad 27,04 \% \quad 10,60 \%$

Cruzeiro $3 \times 1$ Palmeiras

\begin{tabular}{|c|c|c|c|c|c|c|c|c|}
\hline SD 0 & 1,3 & 1,3 & 1,309 & 1,309 & 0 & $36,62 \%$ & $26,34 \%$ & $37,04 \%$ \\
\hline SD I & 1,3 & 1,464 & 1,309 & 1,473 & $-0,164$ & & & \\
\hline "Chance" I & 1,198 & 1,198 & 1,279 & 1,279 & 0 & $34,50 \%$ & $27,12 \%$ & $38,38 \%$ \\
\hline "Chance" II & 1,649 & 1,234 & 1,763 & 1,348 & 0,415 & $34,09 \%$ & $26,45 \%$ & $39,47 \%$ \\
\hline Implícito I & 1,9 & 1,9 & 1,7 & 1,7 & 0 & $43,13 \%$ & $21,83 \%$ & $35,04 \%$ \\
\hline Implícito II & 1,9 & 0,826 & 1,7 & 0,626 & 1,074 & $37,33 \%$ & $37,18 \%$ & $25,49 \%$ \\
\hline Vasco 0x1 Vitória & & & & & & & & \\
\hline SD 0 & 2,059 & 2,059 & 1,017 & 1,017 & 0 & $61,40 \%$ & $20,68 \%$ & $17,92 \%$ \\
\hline $\mathrm{SD} I$ & 2,059 & 2,335 & 1,017 & 1,293 & $-0,276$ & & & \\
\hline "Chance" I & 2,293 & 2,293 & 0,947 & 0,947 & 0 & $67,59 \%$ & $18,32 \%$ & $14,09 \%$ \\
\hline "Chance" II & 2,551 & 2,136 & 1,362 & 0,947 & 0,415 & $64,62 \%$ & $19,73 \%$ & $15,64 \%$ \\
\hline Implícito I & 2 & 2 & 1,4 & 1,4 & 0 & $51,48 \%$ & $21,66 \%$ & $26,86 \%$ \\
\hline Implícito II & 2 & 1,126 & 1,4 & 0,526 & 0,874 & $50,48 \%$ & $32,32 \%$ & $17,20 \%$ \\
\hline
\end{tabular}


Flamengo 2x1 Guarani

$\begin{array}{lrrrrrrrr}\text { SD 0 } & 1,809 & 1,809 & 1,399 & 1,399 & 0 & 47,25 \% & 22,89 \% & 29,86 \% \\ \text { SD I } & 1,809 & 1,974 & 1,399 & 1,564 & -0,165 & & & \\ \text { "Chance" I } & 1,896 & 1,896 & 1,329 & 1,329 & 0 & 50,73 \% & 22,38 \% & 26,89 \% \\ \text { "Chance" II } & 2,263 & 1,848 & 1,801 & 1,386 & 0,415 & 48,41 \% & 22,66 \% & 28,93 \% \\ \text { Implícito I } & 2 & 2 & 1,3 & 1,3 & 0 & 53,67 \% & 21,64 \% & 24,70 \% \\ \text { Implícito II } & 2 & 1,226 & 1,3 & 0,526 & 0,774 & 53,62 \% & 30,47 \% & 15,91 \%\end{array}$

São Paulo 1x2 Corinthians

$\begin{array}{lrrrrrrrr}\text { SD 0 } & 1,536 & 1,536 & 1,446 & 1,446 & 0 & 39,81 \% & 24,36 \% & 35,84 \% \\ \text { SD I } & 1,536 & 1,356 & 1,446 & 1,266 & 0,18 & 38,98 \% & 26,23 \% & 34,78 \% \\ \text { "Chance" I } & 1,681 & 1,681 & 1,588 & 1,588 & 0 & 40,40 \% & 23,12 \% & 36,48 \% \\ \text { "Chance" II } & 2,101 & 1,686 & 2,013 & 1,599 & 0,415 & 40,32 \% & 23,06 \% & 36,62 \% \\ \text { Implícito I } & 1 & 1 & 2,4 & 2,4 & 0 & 14,03 \% & 17,65 \% & 68,32 \% \\ \text { Implícito II } & 1 & 0,126 & 2,4 & 1,526 & 0,874 & 2,82 \% & 23,04 \% & 74,14 \%\end{array}$

Portuguesa $1 \times 1$ Internacional

$\begin{array}{lrrrrrrrr}\text { SD 0 } & 1,678 & 1,678 & 1,054 & 1,054 & 0 & 51,96 \% & 24,23 \% & 23,81 \% \\ \text { SD I } & 1,678 & 1,128 & 1,054 & 0,504 & 0,55 & 51,18 \% & 32,34 \% & 16,48 \% \\ \text { "Chance" I } & 1,583 & 1,583 & 1,034 & 1,034 & 0 & 50,09 \% & 25,14 \% & 24,78 \% \\ \text { "Chance" II } & 2,067 & 1,652 & 1,428 & 1,013 & 0,415 & 52,32 \% & 24,48 \% & 23,20 \% \\ \text { Implícito I } & 1,4 & 1,4 & 1,1 & 1,1 & 0 & 43,80 \% & 26,63 \% & 29,56 \% \\ \text { Implícito II } & 1,4 & 1,308 & 1,1 & 1,008 & 0,092 & 43,43 \% & 27,85 \% & 28,72 \%\end{array}$

Implícito II $\quad 1,4 \quad 1,308 \quad 1,1 \quad 1,008 \quad 0,092 \quad 43,43 \% \quad 27,85 \%$

Santos 1×2 Paraná

$\begin{array}{lrrrrrrrr}\text { SD 0 } & 2,148 & 2,148 & 0,549 & 0,549 & 0 & 74,52 \% & 17,36 \% & 8,12 \% \\ \text { SD I } & 2,148 & 2,329 & 0,549 & 0,731 & -0,182 & & & \\ \text { "Chance" I } & 2,202 & 2,202 & 0,709 & 0,709 & 0 & 71,55 \% & 17,89 \% & 10,56 \% \\ \text { "Chance" II } & 2,501 & 2,086 & 0,975 & 0,56 & 0,415 & 73,13 \% & 18,14 \% & 8,73 \% \\ \text { Implícito I } & 2,4 & 2,4 & 0,9 & 0,9 & 0 & 70,56 \% & 17,14 \% & 12,29 \% \\ \text { Implícito II } & 2,4 & 1,759 & 0,9 & 0,259 & 0,641 & 75,07 \% & 20,07 \% & 4,86 \%\end{array}$

América MG 2x1 Atlético PR

$\begin{array}{lrrrrrrrr}\text { SD 0 } & 1,426 & 1,426 & 1,495 & 1,495 & 0 & 36,14 \% & 24,66 \% & 39,21 \% \\ \text { SD I } & 1,426 & 1,23 & 1,495 & 1,299 & 0,196 & 34,96 \% & 26,80 \% & 38,23 \% \\ \text { "Chance" I } & 1,283 & 1,283 & 1,397 & 1,397 & 0 & 34,44 \% & 25,88 \% & 39,68 \% \\ \text { "Chance" II } & 1,734 & 1,319 & 1,887 & 1,472 & 0,415 & 33,91 \% & 25,24 \% & 40,85 \% \\ \text { Implícito I } & 1,4 & 1,4 & 2 & 2 & 0 & 26,86 \% & 21,66 \% & 51,48 \% \\ \text { Implícito II } & 1,4 & 0,408 & 2 & 1,008 & 0,992 & 14,73 \% & 35,32 \% & 49,95 \%\end{array}$

Coritiba 1x1 Atlético MG

\begin{tabular}{|c|c|c|c|c|c|c|c|c|}
\hline $\mathrm{SD} 0$ & 1,51 & 1,51 & 1,264 & 1,264 & 0 & $42,99 \%$ & $25,20 \%$ & $31,81 \%$ \\
\hline $\mathrm{SD} I$ & 1,51 & 0,733 & 1,264 & 0,487 & 0,776 & $37,23 \%$ & $41,02 \%$ & $21,75 \%$ \\
\hline "Chance" I & 1,502 & 1,502 & 1,108 & 1,108 & 0 & $46,30 \%$ & $25,73 \%$ & $27,98 \%$ \\
\hline "Chance" II & 1,936 & 1,521 & 1,541 & 1,126 & 0,415 & $46,35 \%$ & $25,52 \%$ & $28,12 \%$ \\
\hline Implícito I & 2 & 2 & 1,5 & 1,5 & 0 & $49,36 \%$ & $21,62 \%$ & $29,03 \%$ \\
\hline Implícito II & 2 & 1,159 & 1,5 & 0,659 & 0,841 & $47,83 \%$ & $31,21 \%$ & $20,96 \%$ \\
\hline
\end{tabular}


Grêmio 2x1 América RN

$\begin{array}{lrrrrrrrr}\text { SD 0 } & 1,94 & 1,94 & 1,119 & 1,119 & 0 & 56,47 \% & 21,94 \% & 21,59 \% \\ \text { SD I } & 1,94 & 2,276 & 1,119 & 1,455 & -0,336 & & & \\ \text { "Chance" I } & 1,9 & 1,9 & 1,055 & 1,055 & 0 & 57,10 \% & 22,20 \% & 20,70 \% \\ \text { "Chance" II } & 2,342 & 1,927 & 1,482 & 1,067 & 0,415 & 57,41 \% & 21,98 \% & 20,61 \% \\ \text { Implícito I } & 2,4 & 2,4 & 1,1 & 1,1 & 0 & 66,10 \% & 18,08 \% & 15,82 \% \\ \text { Implícito II } & 2,4 & 1,559 & 1,1 & 0,259 & 0,841 & 70,72 \% & 23,48 \% & 5,79 \%\end{array}$

Goiás 2x2 Juventude

$\begin{array}{lrrrrrrrr}\text { SD 0 } & 1,12 & 1,12 & 0,881 & 0,881 & 0 & 40,97 \% & 30,54 \% & 28,49 \% \\ \text { SD I } & 1,12 & 0,644 & 0,881 & 0,405 & 0,476 & 35,51 \% & 44,78 \% & 19,71 \% \\ \text { "Chance" I } & 1,066 & 1,066 & 0,986 & 0,986 & 0 & 36,90 \% & 30,34 \% & 32,76 \% \\ \text { "Chance" II } & 1,515 & 1,1 & 1,415 & 1 & 0,415 & 37,63 \% & 29,90 \% & 32,47 \% \\ \text { Implícito I } & 1,5 & 1,5 & 1,4 & 1,4 & 0 & 39,86 \% & 24,74 \% & 35,40 \% \\ \text { Implícito II } & 1,5 & 0,903 & 1,4 & 0,803 & 0,597 & 35,83 \% & 33,92 \% & 30,25 \%\end{array}$

Ponte Preta 2x2 Bragantino

\begin{tabular}{|c|c|c|c|c|c|c|c|c|}
\hline SD 0 & 1,455 & 1,455 & 0,977 & 0,977 & 0 & $48,17 \%$ & $26,48 \%$ & $25,35 \%$ \\
\hline $\mathrm{SD} I$ & 1,455 & 1,254 & 0,977 & 0,776 & 0,201 & $47,61 \%$ & $29,38 \%$ & $23,01 \%$ \\
\hline "Chance" I & 1,083 & 1,083 & 0,758 & 0,758 & 0 & $42,87 \%$ & $31,82 \%$ & $25,32 \%$ \\
\hline "Chance" II & 1,58 & 1,165 & 1,122 & 0,707 & 0,415 & $46,73 \%$ & $30,91 \%$ & $22,36 \%$ \\
\hline Implícito I & 1,9 & 1,9 & 1,4 & 1,4 & 0 & $49,28 \%$ & $22,31 \%$ & $28,41 \%$ \\
\hline Implícito II & 1,9 & 0,903 & 1,4 & 0,403 & 0,997 & $46,25 \%$ & $37,90 \%$ & $15,85 \%$ \\
\hline
\end{tabular}

Implícito II $\quad 1,9 \quad 0,903 \quad 1,4 \quad 0,403 \quad 0,997 \quad 46,25 \% \quad 37,90 \% \quad 15,85 \%$

América RN 2×2 Santos

$\begin{array}{lrrrrrrrr}\text { SD 0 } & 1,228 & 1,228 & 1,901 & 1,901 & 0 & 24,55 \% & 22,35 \% & 53,10 \% \\ \text { SD I } & 1,228 & 1,811 & 1,901 & 2,484 & -0,583 & & & \\ \text { "Chance" I } & 1,179 & 1,179 & 1,954 & 1,954 & 0 & 22,71 \% & 21,90 \% & 55,38 \% \\ \text { "Chance" II } & 1,596 & 1,21 & 2,379 & 1,993 & 0,386 & 22,85 \% & 21,62 \% & 55,53 \% \\ \text { Implícito I } & 1,2 & 1,2 & 2,4 & 2,4 & 0 & 17,65 \% & 18,45 \% & 63,90 \% \\ \text { Implícito II } & 1,2 & 0,259 & 2,4 & 1,459 & 0,941 & 6,33 \% & 25,39 \% & 68,28 \%\end{array}$

Implícito II $\quad 1,2 \quad 0,259 \quad 2,4 \quad 1,459 \quad 0,941 \quad 6,33 \% \quad 25,39 \% \quad 68,28 \%$

Bragantino $1 \times 0$ Juventude

$\begin{array}{lrrrrrrrr}\text { SD 0 } & 0,9 & 0,9 & 1,206 & 1,206 & 0 & 27,47 \% & 29,43 \% & 43,09 \% \\ \text { SD I } & 0,9 & 0,72 & 1,206 & 1,026 & 0,18 & 25,12 \% & 32,91 \% & 41,97 \% \\ \text { "Chance" I } & 0,871 & 0,871 & 1,155 & 1,155 & 0 & 27,55 \% & 30,18 \% & 42,27 \% \\ \text { "Chance" II } & 1,294 & 0,908 & 1,635 & 1,249 & 0,386 & 26,94 \% & 28,91 \% & 44,15 \% \\ \text { Implícito I } & 1,4 & 1,4 & 1,4 & 1,4 & 0 & 37,36 \% & 25,28 \% & 37,36 \% \\ \end{array}$

Implícito II $\quad 1,4 \quad 1,008 \quad 1,4 \quad 1,008 \quad 0,392 \quad 34,65 \% \quad 30,70 \% \quad 34,65 \%$

Vasco $3 \times 1$ Coritiba

$\begin{array}{lrrrrrrrr}\text { SD 0 } & 1,622 & 1,622 & 0,499 & 0,499 & 0 & 65,12 \% & 23,85 \% & 11,04 \% \\ \text { SD I } & 1,622 & 1,717 & 0,499 & 0,594 & -0,095 & & & \\ \text { "Chance" I } & 1,453 & 1,453 & 0,73 & 0,73 & 0 & 54,43 \% & 26,79 \% & 18,77 \% \\ \text { "Chance" II } & 1,859 & 1,473 & 0,952 & 0,566 & 0,386 & 59,48 \% & 26,35 \% & 14,18 \% \\ \text { Implícito I } & 1,7 & 1,7 & 1,4 & 1,4 & 0 & 44,69 \% & 23,57 \% & 31,74 \% \\ \text { Implícito II } & 1,7 & 1,126 & 1,4 & 0,826 & 0,574 & 42,49 \% & 30,82 \% & 26,69 \%\end{array}$


Internacional 0x0 Botafogo

$\begin{array}{lrrrrrrrr}\text { SD 0 } & 1,674 & 1,674 & 0,951 & 0,951 & 0 & 54,37 \% & 24,26 \% & 21,37 \% \\ \text { SD I } & 1,674 & 0,784 & 0,951 & 0,061 & 0,89 & 52,20 \% & 45,03 \% & 2,77 \% \\ \text { "Chance" I } & 1,637 & 1,637 & 0,972 & 0,972 & 0 & 52,95 \% & 24,64 \% & 22,41 \% \\ \text { "Chance" II } & 2,964 & 1,642 & 2,288 & 0,966 & 1,322 & 53,18 \% & 24,60 \% & 22,19 \% \\ \text { Implícito I } & 2,2 & 2,2 & 1,5 & 1,5 & 0 & 53,56 \% & 20,41 \% & 26,03 \% \\ \text { Implícito II } & 2,2 & 1,026 & 1,5 & 0,326 & 1,174 & 53,08 \% & 35,28 \% & 11,63 \%\end{array}$

Vitória $2 \times 3$ Corinthians

$\begin{array}{lrrrrrrrr}\text { SD 0 } & 1,791 & 1,791 & 1,46 & 1,46 & 0 & 45,54 \% & 22,90 \% & 31,56 \% \\ \text { SD I } & 1,791 & 1,864 & 1,46 & 1,533 & -0,073 & & & \\ \text { "Chance" I } & 1,641 & 1,641 & 1,514 & 1,514 & 0 & 40,95 \% & 23,57 \% & 35,49 \% \\ \text { "Chance" II } & 2,987 & 1,665 & 2,885 & 1,563 & 1,322 & 40,50 \% & 23,28 \% & 36,17 \% \\ \text { Implícito I } & 1,2 & 1,2 & 1,5 & 1,5 & 0 & 30,37 \% & 25,48 \% & 44,15 \% \\ \text { Implícito II } & 1,2 & 1,026 & 1,5 & 1,326 & 0,174 & 28,89 \% & 27,60 \% & 43,51 \%\end{array}$

Santos 3x1 Goiás

$\begin{array}{lrrrrrrrr}\text { SD 0 } & 2,092 & 2,092 & 0,781 & 0,781 & 0 & 67,75 \% & 19,42 \% & 12,83 \% \\ \text { SD I } & 2,092 & 2,429 & 0,781 & 1,119 & -0,337 & & & \\ \text { "Chance" I } & 2,342 & 2,342 & 0,891 & 0,891 & 0 & 69,76 \% & 17,60 \% & 12,63 \% \\ \text { "Chance" II } & 3,502 & 2,179 & 2,147 & 0,825 & 1,322 & 68,24 \% & 18,77 \% & 12,88 \% \\ \text { Implícito I } & 2,1 & 2,1 & 1,2 & 1,2 & 0 & 58,03 \% & 20,76 \% & 21,21 \% \\ \text { Implícito II } & 2,1 & 1,326 & 1,2 & 0,426 & 0,774 & 59,53 \% & 28,62 \% & 11,85 \%\end{array}$

Implícito II $\quad 2,1 \quad 1,326 \quad 1,2 \quad 0,426 \quad 0,774 \quad 59,53 \% \quad 28,62 \% \quad 11,85 \%$

Palmeiras 3x2 Paraná

$\begin{array}{lrrrrrrrr}\text { SD 0 } & 2,186 & 2,186 & 0,494 & 0,494 & 0 & 76,52 \% & 16,52 \% & 6,96 \% \\ \text { SD I } & 2,186 & 2,273 & 0,494 & 0,581 & -0,087 & & & \\ \text { "Chance" I } & 2,187 & 2,187 & 0,664 & 0,664 & 0 & 72,39 \% & 17,75 \% & 9,86 \% \\ \text { "Chance" II } & 3,408 & 2,086 & 1,792 & 0,469 & 1,322 & 75,36 \% & 17,45 \% & 7,11 \% \\ \text { Implícito I } & 2,6 & 2,6 & 1 & 1 & 0 & 71,68 \% & 16,03 \% & 12,28 \% \\ \text { Implícito II } & 2,6 & 1,659 & 1 & 0,059 & 0,941 & 79,10 \% & 19,74 \% & 1,15 \%\end{array}$

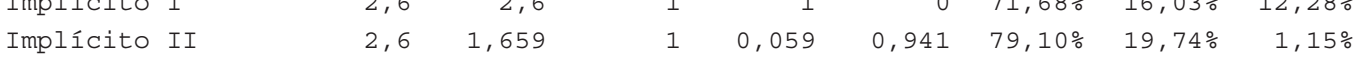

Portuguesa $3 \times 1$ Bragantino

$\begin{array}{lrrrrrrrr}\text { SD 0 } & 2,293 & 2,293 & 0,593 & 0,593 & 0 & 75,88 \% & 16,17 \% & 7,95 \% \\ \text { SD I } & 2,293 & 2,287 & 0,593 & 0,587 & 0,006 & 75,92 \% & 16,19 \% & 7,89 \% \\ \text { "Chance" I } & 2,456 & 2,456 & 0,693 & 0,693 & 0 & 76,15 \% & 15,34 \% & 8,50 \% \\ \text { "Chance" II } & 3,585 & 2,263 & 1,897 & 0,575 & 1,322 & 75,70 \% & 16,34 \% & 7,84 \% \\ \text { Implícito I } & 2 & 2 & 1,1 & 1,1 & 0 & 58,21 \% & 21,40 \% & 20,39 \% \\ \text { Implírito } & 2 & 1,426 & 1,1 & 0,526 & 0,574 & 59,36 \% & 27,02 \% & 13,61 \%\end{array}$

Implícito II $\quad 2 \quad 1,426 \quad 1,1 \quad 0,526 \quad 0,574 \quad 59,36 \% \quad 27,02 \% \quad 13,61 \%$

Guarani $1 \times 3$ Grêmio

\begin{tabular}{|c|c|c|c|c|c|c|c|c|}
\hline SD 0 & 1,916 & 1,916 & 1,01 & 1,01 & 0 & $58,52 \%$ & $21,98 \%$ & $19,51 \%$ \\
\hline $\mathrm{SD} I$ & 1,916 & 2,105 & 1,01 & 1,199 & $-0,189$ & & & \\
\hline "Chance" I & 1,889 & 1,889 & 1,042 & 1,042 & 0 & $57,15 \%$ & $22,28 \%$ & $20,56 \%$ \\
\hline "Chance" II & 3,172 & 1,849 & 2,357 & 1,035 & 1,322 & $56,39 \%$ & $22,63 \%$ & $20,92 \%$ \\
\hline Implícito I & 1,2 & 1,2 & 1,4 & 1,4 & 0 & $32,21 \%$ & $26,23 \%$ & $41,56 \%$ \\
\hline Implícito II & 1,2 & 1,008 & 1,4 & 1,208 & 0,192 & $30,58 \%$ & $28,82 \%$ & $40,60 \%$ \\
\hline
\end{tabular}


Atlético PR 1x2 Cruzeiro

$\begin{array}{lrrrrrrrr}\text { SD 0 } & 1,451 & 1,451 & 1,133 & 1,133 & 0 & 44,39 \% & 26,09 \% & 29,52 \% \\ \text { SD I } & 1,451 & 1,45 & 1,133 & 1,132 & 0,001 & 44,39 \% & 26,09 \% & 29,51 \% \\ \text { "Chance" I } & 1,437 & 1,437 & 1,093 & 1,093 & 0 & 44,96 \% & 26,33 \% & 28,70 \% \\ \text { "Chance" II } & 2,774 & 1,452 & 2,4 & 1,078 & 1,322 & 45,67 \% & 26,25 \% & 28,06 \% \\ \text { Implícito I } & 1,4 & 1,4 & 1,4 & 1,4 & 0 & 37,36 \% & 25,28 \% & 37,36 \% \\ \text { Implícito II } & 1,4 & 1,008 & 1,4 & 1,008 & 0,392 & 34,65 \% & 30,70 \% & 34,65 \%\end{array}$

Flamengo 2x0 América MG

$\begin{array}{lrrrrrrrr}\text { SD D } & 2,049 & 2,049 & 1,145 & 1,145 & 0 & 58,21 \% & 21,07 \% & 20,71 \% \\ \text { SD I } & 2,049 & 2,029 & 1,145 & 1,125 & 0,02 & 58,25 \% & 21,20 \% & 20,55 \% \\ \text { "Chance" I } & 2,094 & 2,094 & 1,068 & 1,068 & 0 & 60,92 \% & 20,52 \% & 18,56 \% \\ \text { "Chance" II } & 3,343 & 2,02 & 2,423 & 1,101 & 1,322 & 58,56 \% & 21,23 \% & 20,14 \% \\ \text { Implícito I } & 2 & 2 & 1,2 & 1,2 & 0 & 55,91 \% & 21,55 \% & 22,54 \% \\ \text { Implícito II } & 2 & 1,459 & 1,2 & 0,659 & 0,541 & 56,50 \% & 26,68 \% & 16,81 \%\end{array}$

América RN 0x1 Ponte Preta

$\begin{array}{lrrrrrrrr}\text { SD 0 } & 1,503 & 1,503 & 1,268 & 1,268 & 0 & 42,73 \% & 25,23 \% & 32,04 \% \\ \text { SD I } & 1,503 & 0,898 & 1,268 & 0,663 & 0,605 & 39,02 \% & 35,49 \% & 25,48 \% \\ \text { "Chance" I } & 1,442 & 1,442 & 1,222 & 1,222 & 0 & 42,17 \% & 25,84 \% & 31,99 \% \\ \text { "Chance" II } & 2,794 & 1,472 & 2,66 & 1,338 & 1,322 & 40,43 \% & 25,16 \% & 34,38 \% \\ \text { Implícito I } & 1,4 & 1,4 & 1,3 & 1,3 & 0 & 39,41 \% & 25,78 \% & 34,81 \% \\ \text { Implícito II } & 1,4 & 1,108 & 1,3 & 1,008 & 0,292 & 37,67 \% & 29,77 \% & 32,56 \%\end{array}$

Atlético MG $1 \times 0$ sport

$\begin{array}{lrrrrrrrr}\text { SD 0 } & 1,678 & 1,678 & 1,301 & 1,301 & 0 & 46,31 \% & 23,93 \% & 29,76 \% \\ \text { SD I } & 1,678 & 1,176 & 1,301 & 0,799 & 0,503 & 44,69 \% & 30,33 \% & 24,98 \% \\ \text { "Chance" I } & 1,6 & 1,6 & 1,302 & 1,302 & 0 & 44,42 \% & 24,46 \% & 31,12 \% \\ \text { "Chance" II } & 2,961 & 1,639 & 2,658 & 1,335 & 1,322 & 44,60 \% & 24,11 \% & 31,25 \% \\ \text { Implícito I } & 1,5 & 1,5 & 1,4 & 1,4 & 0 & 39,86 \% & 24,74 \% & 35,40 \% \\ \text { Implícito II } & 1,5 & 0,903 & 1,4 & 0,803 & 0,597 & 35,83 \% & 33,92 \% & 30,25 \%\end{array}$

Implícito II $\quad 1,5 \quad 0,903 \quad 1,4 \quad 0,803 \quad 0,597 \quad 35,83 \% \quad 33,92 \% \quad 30,25 \%$

Juventude $2 \times 1$ São Paulo

$\begin{array}{lrrrrrrrr}\text { SD 0 } & 1,223 & 1,223 & 1,053 & 1,053 & 0 & 40,01 \% & 28,41 \% & 31,58 \% \\ \text { SD I } & 1,223 & 0,237 & 1,053 & 0,068 & 0,986 & 19,91 \% & 74,90 \% & 5,19 \% \\ \text { "Chance" I } & 1,139 & 1,139 & 1,052 & 1,052 & 0 & 37,62 \% & 29,17 \% & 33,21 \% \\ \text { "Chance" II } & 1,577 & 1,181 & 1,453 & 1,057 & 0,396 & 38,71 \% & 28,76 \% & 32,53 \% \\ \text { Implícito I } & 1,4 & 1,4 & 1,3 & 1,3 & 0 & 39,41 \% & 25,78 \% & 34,81 \% \\ \text { Implícito II } & 1,4 & 1,003 & 1,3 & 0,903 & 0,397 & 36,81 \% & 31,72 \% & 31,47 \%\end{array}$

Implícito II $1,4 \quad 1,003 \quad 1,3 \quad 0,903 \quad 0,397 \quad 36,81 \% \quad 31,72 \% \quad 31,47 \%$

Vasco $2 \times 1$ Grêmio

$\begin{array}{lrrrrrrrr}\text { SD 0 } & 2,047 & 2,047 & 0,589 & 0,589 & 0 & 71,68 \% & 18,78 \% & 9,53 \% \\ \text { SD I } & 2,047 & 2,125 & 0,589 & 0,667 & -0,078 & & & \\ \text { "Chance" I } & 1,972 & 1,972 & 0,772 & 0,772 & 0 & 65,58 \% & 20,64 \% & 13,78 \% \\ \text { "Chance" II } & 2,295 & 1,899 & 1,039 & 0,643 & 0,396 & 67,35 \% & 20,84 \% & 11,80 \% \\ \text { Implícito I } & 2 & 2 & 1,4 & 1,4 & 0 & 51,48 \% & 21,66 \% & 26,86 \% \\ \text { Implícito II } & 2 & 1,126 & 1,4 & 0,526 & 0,874 & 50,48 \% & 32,32 \% & 17,20 \%\end{array}$


Palmeiras $2 \times 1$ Vasco

$\begin{array}{lrrrrrrrr}\text { SD 0 } & 1,707 & 1,707 & 0,996 & 0,996 & 0 & 54,06 \% & 23,95 \% & 21,99 \% \\ \text { SD I } & 1,707 & 1,835 & 0,996 & 1,124 & -0,128 & & & \\ \text { "Chance" I } & 1,569 & 1,569 & 1,021 & 1,021 & 0 & 50,04 \% & 25,28 \% & 24,67 \% \\ \text { "Chance" II } & 2,064 & 1,667 & 1,402 & 1,006 & 0,396 & 52,86 \% & 24,34 \% & 22,79 \% \\ \text { Implícito I } & 2 & 2 & 1,5 & 1,5 & 0 & 49,36 \% & 21,62 \% & 29,03 \% \\ \text { Implícito II } & 2 & 1,159 & 1,5 & 0,659 & 0,841 & 47,83 \% & 31,21 \% & 20,96 \%\end{array}$

Goiás 2×2 Vasco

$\begin{array}{lrrrrrrrr}\text { SD 0 } & 1,1 & 1,1 & 1,396 & 1,396 & 0 & 29,63 \% & 26,67 \% & 43,70 \% \\ \text { SD I } & 1,1 & 1,291 & 1,396 & 1,587 & -0,191 & & & \\ \text { "Chance" I } & 1,039 & 1,039 & 1,448 & 1,448 & 0 & 27,09 \% & 26,39 \% & 46,51 \% \\ \text { "Chance" II } & 1,462 & 1,065 & 1,895 & 1,498 & 0,396 & 26,95 \% & 25,87 \% & 47,19 \% \\ \text { Implícito I } & 1,3 & 1,3 & 1,6 & 1,6 & 0 & 31,09 \% & 24,47 \% & 44,44 \% \\ \text { Implícito II } & 1,3 & 0,808 & 1,6 & 1,108 & 0,492 & 26,46 \% & 31,16 \% & 42,38 \%\end{array}$

Paraná 2x1 Flamengo

$\begin{array}{lrrrrrrrr}\text { SD 0 } & 1,519 & 1,519 & 1,4 & 1,4 & 0 & 40,33 \% & 24,63 \% & 35,03 \% \\ \text { SD I } & 1,519 & 1,487 & 1,4 & 1,368 & 0,032 & 40,20 \% & 24,95 \% & 34,85 \% \\ \text { "Chance" I } & 1,338 & 1,338 & 1,345 & 1,345 & 0 & 36,88 \% & 25,92 \% & 37,21 \% \\ \text { "Chance" II } & 1,823 & 1,427 & 1,782 & 1,386 & 0,396 & 38,32 \% & 25,21 \% & 36,47 \% \\ \text { Implícito I } & 1,4 & 1,4 & 1,9 & 1,9 & 0 & 28,41 \% & 22,31 \% & 49,28 \% \\ \text { Implícito II } & 1,4 & 0,508 & 1,9 & 1,008 & 0,892 & 18,23 \% & 34,73 \% & 47,04 \%\end{array}$

Implícito II $\quad 1,4 \quad 0,508 \quad 1,9 \quad 1,008 \quad 0,892 \quad 18,23 \% \quad 34,73 \%$

Botafogo $2 \times 1$ Santos

\begin{tabular}{|c|c|c|c|c|c|c|c|c|}
\hline SD 0 & 1,611 & 1,611 & 1,474 & 1,474 & 0 & $41,04 \%$ & $23,86 \%$ & $35,10 \%$ \\
\hline $\mathrm{SD} I$ & 1,611 & 1,102 & 1,474 & 0,965 & 0,509 & $38,46 \%$ & $30,15 \%$ & $31,39 \%$ \\
\hline "Chance" I & 1,592 & 1,592 & 1,45 & 1,45 & 0 & $41,07 \%$ & $24,04 \%$ & $34,89 \%$ \\
\hline "Chance" II & 1,997 & 1,601 & 1,908 & 1,512 & 0,396 & $40,03 \%$ & $23,77 \%$ & $36,20 \%$ \\
\hline Implícito I & 1,4 & 1,4 & 2 & 2 & 0 & $26,86 \%$ & $21,66 \%$ & $51,48 \%$ \\
\hline Implícito II & 1,4 & 0,526 & 2 & 1,126 & 0,874 & $17,20 \%$ & $32,32 \%$ & $50,48 \%$ \\
\hline
\end{tabular}

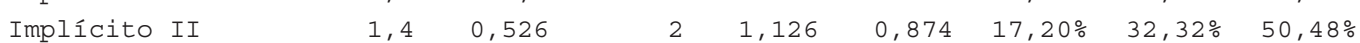

América MG $1 \times 1$ Palmeiras

$\begin{array}{lrrrrrrrr}\text { SD 0 } & 1,167 & 1,167 & 1,847 & 1,847 & 0 & 23,96 \% & 22,76 \% & 53,28 \% \\ \text { SD I } & 1,167 & 1,277 & 1,847 & 1,957 & -0,11 & & \\ \text { "Chance" I } & 1,087 & 1,087 & 1,767 & 1,767 & 0 & 23,28 \% & 23,42 \% & 53,29 \% \\ \text { "Chance" II } & 1,511 & 1,114 & 2,238 & 1,841 & 0,396 & 22,85 \% & 22,78 \% & 54,37 \% \\ \text { Implícito I } & 1,3 & 1,3 & 2 & 2 & 0 & 24,70 \% & 21,64 \% & 53,67 \% \\ \text { Implícito II } & 1,3 & 0,526 & 2 & 1,226 & 0,774 & 15,91 \% & 30,47 \% & 53,62 \%\end{array}$

Corinthians 2x1 América RN

$\begin{array}{lrrrrrrrr}\text { SD 0 } & 2,238 & 2,238 & 0,735 & 0,735 & 0 & 71,57 \% & 17,69 \% & 10,74 \% \\ \text { SD I } & 2,238 & 2,331 & 0,735 & 0,828 & -0,093 & & & \\ \text { "Chance" I } & 2,39 & 2,39 & 0,824 & 0,824 & 0 & 72,12 \% & 16,80 \% & 11,08 \% \\ \text { "Chance" II } & 2,624 & 2,228 & 1,117 & 0,72 & 0,396 & 71,72 \% & 17,70 \% & 10,56 \% \\ \text { Implícito I } & 2 & 2 & 1 & 1 & 0 & 60,57 \% & 21,17 \% & 18,26 \% \\ \text { Implícito II } & 2 & 1,813 & 1 & 0,813 & 0,187 & 61,09 \% & 22,54 \% & 16,37 \%\end{array}$


São Paulo 2x0 Atlético PR

$\begin{array}{lrrrrrrrr}\text { SD 0 } & 1,859 & 1,859 & 1,098 & 1,098 & 0 & 55,16 \% & 22,62 \% & 22,23 \% \\ \text { SD I } & 1,859 & 1,585 & 1,098 & 0,824 & 0,274 & 55,38 \% & 25,17 \% & 19,45 \% \\ \text { "Chance" I } & 1,795 & 1,795 & 1,083 & 1,083 & 0 & 54,06 \% & 23,17 \% & 22,77 \% \\ \text { "Chance" II } & 2,154 & 1,757 & 1,466 & 1,07 & 0,396 & 53,47 \% & 23,50 \% & 23,03 \% \\ \text { Implícito I } & 1,6 & 1,6 & 1,2 & 1,2 & 0 & 46,66 \% & 24,71 \% & 28,63 \% \\ \text { Implícito II } & 1,6 & 1,103 & 1,2 & 0,703 & 0,497 & 44,89 \% & 31,87 \% & 23,23 \%\end{array}$

Grêmio 4x2 Portuguesa

$\begin{array}{lrrrrrrrr}\text { SD 0 } & 1,432 & 1,432 & 1,737 & 1,737 & 0 & 31,84 \% & 23,27 \% & 44,88 \% \\ \text { SD I } & 1,432 & 1,29 & 1,737 & 1,595 & 0,142 & 30,93 \% & 24,53 \% & 44,53 \% \\ \text { "Chance" I } & 1,357 & 1,357 & 1,591 & 1,591 & 0 & 32,65 \% & 24,36 \% & 42,98 \% \\ \text { "Chance" II } & 1,785 & 1,389 & 2,033 & 1,637 & 0,396 & 32,59 \% & 23,98 \% & 43,43 \% \\ \text { Implícito I } & 1,6 & 1,6 & 1,4 & 1,4 & 0 & 42,31 \% & 24,17 \% & 33,52 \% \\ \text { Implícito II } & 1,6 & 1,259 & 1,4 & 1,059 & 0,341 & 40,89 \% & 28,05 \% & 31,06 \%\end{array}$

Bragantino 0x0 Vitória

$\begin{array}{lrrrrrrrr}\text { SD 0 } & 1,451 & 1,451 & 1,766 & 1,766 & 0 & 31,78 \% & 23,06 \% & 45,16 \% \\ \text { SD I } & 1,451 & 2,093 & 1,766 & 2,408 & -0,642 & & & \\ \text { "Chance" I } & 1,377 & 1,377 & 1,642 & 1,642 & 0 & 32,20 \% & 23,98 \% & 43,82 \% \\ \text { "Chance" II } & 1,944 & 1,548 & 2,114 & 1,718 & 0,396 & 34,87 \% & 23,07 \% & 42,06 \% \\ \text { Implícito I } & 1,4 & 1,4 & 1,5 & 1,5 & 0 & 35,40 \% & 24,74 \% & 39,86 \% \\ \text { Implícito II } & 1,4 & 0,908 & 1,5 & 1,008 & 0,492 & 31,53 \% & 31,61 \% & 36,86 \%\end{array}$

Implícito II $\quad 1,4 \quad 0,908 \quad 1,5 \quad 1,008 \quad 0,492 \quad 31,53 \% \quad 31,61 \% 36,86 \%$

Sport 2x1 Guarani

$\begin{array}{lrrrrrrrr}\text { SD 0 } & 1,733 & 1,733 & 0,989 & 0,989 & 0 & 54,85 \% & 23,70 \% & 21,45 \% \\ \text { SD I } & 1,733 & 2,014 & 0,989 & 1,27 & -0,281 & & & \\ \text { "Chance" I } & 1,865 & 1,865 & 0,978 & 0,978 & 0 & 58,14 \% & 22,40 \% & 19,45 \% \\ \text { "Chance" II } & 2,226 & 1,829 & 1,35 & 0,953 & 0,396 & 57,96 \% & 22,70 \% & 19,34 \% \\ \text { Implícito I } & 1,4 & 1,4 & 1,1 & 1,1 & 0 & 43,80 \% & 26,63 \% & 29,56 \% \\ \text { Implícito II } & 1,4 & 1,308 & 1,1 & 1,008 & 0,092 & 43,43 \% & 27,85 \% & 28,72 \%\end{array}$

Implícito II $1,4 \quad 1,308 \quad 1,1 \quad 1,008 \quad 0,092 \quad 43,43 \% \quad 27,85 \% \quad 28,72 \%$

Ponte Preta 0x0 Atlético MG

\begin{tabular}{lrrrrrrrr} 
SD 0 & 1,357 & 1,357 & 1,355 & 1,355 & 0 & $37,17 \%$ & $25,75 \%$ & $37,08 \%$ \\
SD I & 1,357 & $-0,285$ & 1,355 & $-0,287$ & 1,642 & & & \\
"Chance" I & 1,283 & 1,283 & 1,333 & 1,333 & 0 & $35,70 \%$ & $26,28 \%$ & $38,02 \%$ \\
"Chance" II & 1,719 & 1,323 & 1,757 & 1,36 & 0,396 & $36,18 \%$ & $25,91 \%$ & $37,91 \%$ \\
Implícito I & 1,4 & 1,4 & 1,7 & 1,7 & 0 & $31,74 \%$ & $23,57 \%$ & $44,69 \%$ \\
\hline
\end{tabular}

Implícito II $\quad 1,4 \quad 0,826 \quad 1,7 \quad 1,126 \quad 0,574 \quad 26,69 \% \quad 30,82 \% \quad 42,49 \%$

Coritiba $1 \times 0$ Internacional

\begin{tabular}{|c|c|c|c|c|c|c|c|c|}
\hline SD 0 & 1,067 & 1,067 & 0,993 & 0,993 & 0 & $36,78 \%$ & $30,28 \%$ & $32,95 \%$ \\
\hline $\mathrm{SD} I$ & 1,067 & 1,024 & 0,993 & 0,95 & 0,043 & $36,41 \%$ & $31,08 \%$ & $32,51 \%$ \\
\hline "Chance" I & 1,06 & 1,06 & 0,954 & 0,954 & 0 & $37,43 \%$ & $30,67 \%$ & $31,90 \%$ \\
\hline "Chance" II & 1,484 & 1,088 & 1,289 & 0,893 & 0,396 & $39,70 \%$ & $30,82 \%$ & $29,47 \%$ \\
\hline Implícito I & 1,5 & 1,5 & 1,4 & 1,4 & 0 & $39,86 \%$ & $24,74 \%$ & $35,40 \%$ \\
\hline Implícito II & 1,5 & 1,126 & 1,4 & 1,026 & 0,374 & $37,80 \%$ & $29,47 \%$ & $32,72 \%$ \\
\hline
\end{tabular}


Cruzeiro 5x0 Juventude

$\begin{array}{lrrrrrrrr}\text { SD 0 } & 1,392 & 1,392 & 0,561 & 0,561 & 0 & 57,43 \% & 27,62 \% & 14,95 \% \\ \text { SD I } & 1,392 & 1,078 & 0,561 & 0,247 & 0,315 & 57,42 \% & 34,16 \% & 8,43 \% \\ \text { "Chance" I } & 1,307 & 1,307 & 0,769 & 0,769 & 0 & 49,33 \% & 28,70 \% & 21,97 \% \\ \text { "Chance" II } & 1,745 & 1,349 & 1,025 & 0,629 & 0,396 & 54,30 \% & 28,31 \% & 17,39 \% \\ \text { Implícito I } & 1,4 & 1,4 & 1,1 & 1,1 & 0 & 43,80 \% & 26,63 \% & 29,56 \% \\ \text { Implícito II } & 1,4 & 1,308 & 1,1 & 1,008 & 0,092 & 43,43 \% & 27,85 \% & 28,72 \%\end{array}$

Cruzeiro 2x1 Palmeiras

$\begin{array}{lrrrrrrrr}\text { SD 0 } & 1,508 & 1,508 & 1,27 & 1,27 & 0 & 42,81 \% & 25,19 \% & 32,00 \% \\ \text { SD I } & 1,508 & 1,753 & 1,27 & 1,515 & -0,245 & & & \\ \text { "Chance" I } & 1,402 & 1,402 & 1,246 & 1,246 & 0 & 40,61 \% & 26,01 \% & 33,37 \% \\ \text { "Chance" II } & 1,884 & 1,444 & 1,754 & 1,315 & 0,44 & 40,23 \% & 25,44 \% & 34,33 \% \\ \text { Implícito I } & 1,6 & 1,6 & 1,7 & 1,7 & 0 & 36,40 \% & 23,00 \% & 40,61 \% \\ \text { Implícito II } & 1,6 & 0,959 & 1,7 & 1,059 & 0,641 & 32,08 \% & 30,63 \% & 37,29 \%\end{array}$

Grêmio 0x1 Corinthians

$\begin{array}{lrrrrrrrr}\text { SD 0 } & 1,457 & 1,457 & 1,555 & 1,555 & 0 & 35,74 \% & 24,21 \% & 40,04 \% \\ \text { SD I } & 1,457 & 1,359 & 1,555 & 1,457 & 0,098 & 35,20 \% & 25,16 \% & 39,63 \% \\ \text { "Chance" I } & 1,424 & 1,424 & 1,395 & 1,395 & 0 & 38,04 \% & 25,18 \% & 36,78 \% \\ \text { "Chance" II } & 1,887 & 1,447 & 1,877 & 1,437 & 0,44 & 37,80 \% & 24,85 \% & 37,35 \% \\ \text { Implícito I } & 1,4 & 1,4 & 1,6 & 1,6 & 0 & 33,52 \% & 24,17 \% & 42,31 \% \\ \text { Implícito II } & 1,4 & 0,926 & 1,6 & 1,126 & 0,474 & 29,74 \% & 30,17 \% & 40,09 \%\end{array}$

$0,474 \quad 29,74 \% \quad 30,17 \% \quad 40,09 \%$

Sport $3 \times 1$ Santos

$\begin{array}{lrrrrrrrr}\text { SD 0 } & 1,591 & 1,591 & 1,237 & 1,237 & 0 & 45,60 \% & 24,70 \% & 29,70 \% \\ \text { SD I } & 1,591 & 2,052 & 1,237 & 1,698 & -0,461 & & & \\ \text { "Chance" I } & 1,593 & 1,593 & 1,22 & 1,22 & 0 & 46,03 \% & 24,72 \% & 29,25 \% \\ \text { "Chance" II } & 2,049 & 1,609 & 1,729 & 1,29 & 0,44 & 44,88 \% & 24,44 \% & 30,68 \% \\ \text { Implícito I } & 1,4 & 1,4 & 1,4 & 1,4 & 0 & 37,36 \% & 25,28 \% & 37,36 \% \\ \text { Implícito II } & 1,4 & 1,259 & 1,4 & 1,259 & 0,141 & 36,56 \% & 26,89 \% & 36,56 \%\end{array}$

Implícito II $\quad 1,4 \quad 1,259 \quad 1,4 \quad 1,259 \quad 0,141 \quad 36,56 \%$

Portuguesa 3x1 Coritiba

$\begin{array}{lrrrrrrrr}\text { SD 0 } & 1,888 & 1,888 & 0,882 & 0,882 & 0 & 61,03 \% & 21,94 \% & 17,03 \% \\ \text { SD I } & 1,888 & 1,434 & 0,882 & 0,429 & 0,453 & 62,45 \% & 26,63 \% & 10,92 \% \\ \text { "Chance" I } & 1,726 & 1,726 & 0,945 & 0,945 & 0 & 55,76 \% & 23,73 \% & 20,51 \% \\ \text { "Chance" II } & 2,181 & 1,741 & 1,336 & 0,896 & 0,44 & 57,32 \% & 23,51 \% & 19,16 \% \\ \text { Implícito I } & 1,3 & 1,3 & 1,4 & 1,4 & 0 & 34,81 \% & 25,78 \% & 39,41 \% \\ \text { Implícito II } & 1,3 & 1,259 & 1,4 & 1,359 & 0,041 & 34,54 \% & 26,24 \% & 39,21 \%\end{array}$

Palmeiras 2x1 Cruzeiro

$\begin{array}{lrrrrrrrr}\text { SD 0 } & 1,806 & 1,806 & 0,983 & 0,983 & 0 & 56,70 \% & 22,98 \% & 20,32 \% \\ \text { SD I } & 1,806 & 2,101 & 0,983 & 1,278 & -0,295 & & & \\ \text { "Chance" I } & 1,783 & 1,783 & 0,99 & 0,99 & 0 & 55,99 \% & 23,21 \% & 20,80 \% \\ \text { "Chance" II } & 2,238 & 1,8 & 1,407 & 0,969 & 0,439 & 56,90 \% & 23,02 \% & 20,08 \% \\ \text { Implícito I } & 2 & 2 & 1,5 & 1,5 & 0 & 49,36 \% & 21,62 \% & 29,03 \% \\ \text { Implícito II } & 2 & 1,159 & 1,5 & 0,659 & 0,841 & 47,83 \% & 31,21 \% & 20,96 \%\end{array}$


Corinthians 0x2 Grêmio

$\begin{array}{lrrrrrrrr}\text { SD 0 } & 2,069 & 2,069 & 0,84 & 0,84 & 0 & 65,85 \% & 19,94 \% & 14,21 \% \\ \text { SD I } & 2,069 & 1,898 & 0,84 & 0,669 & 0,171 & 66,66 \% & 20,99 \% & 12,36 \% \\ \text { "Chance" I } & 1,979 & 1,979 & 0,937 & 0,937 & 0 & 61,64 \% & 21,19 \% & 17,17 \% \\ \text { "Chance" II } & 2,348 & 1,909 & 1,319 & 0,88 & 0,439 & 61,53 \% & 21,72 \% & 16,75 \% \\ \text { Implícito I } & 1,8 & 1,8 & 1,4 & 1,4 & 0 & 47,02 \% & 22,95 \% & 30,03 \% \\ \text { Implícito II } & 1,8 & 1,126 & 1,4 & 0,726 & 0,674 & 45,02 \% & 31,40 \% & 23,58 \%\end{array}$

Coritiba 0x0 Portuguesa

$\begin{array}{lrrrrrrrr}\text { SD 0 } & 1,433 & 1,433 & 1,394 & 1,394 & 0 & 38,31 \% & 25,14 \% & 36,55 \% \\ \text { SD I } & 1,433 & 1,061 & 1,394 & 1,022 & 0,372 & 35,95 \% & 30,10 \% & 33,94 \% \\ \text { "Chance" I } & 1,363 & 1,363 & 1,247 & 1,247 & 0 & 39,57 \% & 26,28 \% & 34,15 \% \\ \text { "Chance" II } & 1,836 & 1,397 & 1,74 & 1,301 & 0,439 & 39,32 \% & 25,79 \% & 34,89 \% \\ \text { Implícito I } & 2 & 2 & 1,6 & 1,6 & 0 & 47,29 \% & 21,53 \% & 31,18 \% \\ \text { Implícito II } & 2 & 0,926 & 1,6 & 0,526 & 1,074 & 43,62 \% & 36,29 \% & 20,09 \%\end{array}$

Implícito II

Santos $2 \times 1$ sport

$\begin{array}{lrrrrrrrr}\text { SD 0 } & 1,772 & 1,772 & 1,109 & 1,109 & 0 & 52,90 \% & 23,38 \% & 23,72 \% \\ \text { SD I } & 1,772 & 2,266 & 1,109 & 1,603 & -0,494 & & & \\ \text { "Chance" I } & 1,744 & 1,744 & 1,156 & 1,156 & 0 & 51,15 \% & 23,61 \% & 25,24 \% \\ \text { "Chance" II } & 2,212 & 1,773 & 1,612 & 1,174 & 0,439 & 51,43 \% & 23,36 \% & 25,21 \% \\ \text { Implícito I } & 1,7 & 1,7 & 1,1 & 1,1 & 0 & 51,40 \% & 24,01 \% & 24,58 \% \\ \text { Implícito II } & 1,7 & 1,426 & 1,1 & 0,826 & 0,274 & 51,17 \% & 27,08 \% & 21,75 \%\end{array}$

Implícito II $\quad 1,7 \quad 1,426 \quad 1,1 \quad 0,826 \quad 0,274 \quad 51,17 \% \quad 27,08 \% \quad 21,75 \%$

Palmeiras $2 \times 3$ Cruzeiro

$\begin{array}{lrrrrrrrr}\text { SD 0 } & 1,809 & 1,809 & 0,988 & 0,988 & 0 & 56,65 \% & 22,95 \% & 20,40 \% \\ \text { SD I } & 1,809 & 2,144 & 0,988 & 1,323 & -0,335 & & & \\ \text { "Chance" I } & 1,793 & 1,793 & 0,994 & 0,994 & 0 & 56,13 \% & 23,12 \% & 20,75 \% \\ \text { "Chance" II } & 2,245 & 1,807 & 1,413 & 0,975 & 0,438 & 56,91 \% & 22,96 \% & 20,13 \% \\ \text { Implícito I } & 2 & 2 & 1,6 & 1,6 & 0 & 47,29 \% & 21,53 \% & 31,18 \% \\ \text { Implícito II } & 2 & 1,059 & 1,6 & 0,659 & 0,941 & 44,63 \% & 32,82 \% & 22,55 \%\end{array}$

Implícito II $\quad 2 \quad 1,059 \quad 1,6 \quad 0,659 \quad 0,941 \quad 44,63 \% \quad 32,82 \% \quad 22,55 \%$

Coritiba $2 \times 2$ Portuguesa

$\begin{array}{lrrrrrrrr}\text { SD 0 } & 1,368 & 1,368 & 1,334 & 1,334 & 0 & 37,88 \% & 25,80 \% & 36,32 \% \\ \text { SD I } & 1,368 & 0,836 & 1,334 & 0,802 & 0,532 & 33,56 \% & 34,81 \% & 31,63 \% \\ \text { "Chance" I } & 1,303 & 1,303 & 1,202 & 1,202 & 0 & 38,94 \% & 26,93 \% & 34,13 \% \\ \text { "Chance" II } & 1,775 & 1,337 & 1,684 & 1,246 & 0,438 & 38,91 \% & 26,46 \% & 34,63 \% \\ \text { Implícito I } & 1,4 & 1,4 & 1,4 & 1,4 & 0 & 37,36 \% & 25,28 \% & 37,36 \%\end{array}$

Corinthians $1 \times 0$ Grêmio

$\begin{array}{lrrrrrrrr}\text { SD 0 } & 1,974 & 1,974 & 0,896 & 0,896 & 0 & 62,53 \% & 21,11 \% & 16,36 \% \\ \text { SD I } & 1,974 & 1,842 & 0,896 & 0,764 & 0,132 & 63,00 \% & 22,06 \% & 14,95 \% \\ \text { "Chance" I } & 1,87 & 1,87 & 0,974 & 0,974 & 0 & 58,37 \% & 22,34 \% & 19,29 \% \\ \text { "Chance" II } & 2,263 & 1,825 & 1,371 & 0,933 & 0,438 & 58,36 \% & 22,71 \% & 18,93 \% \\ \text { Implícito I } & 1,8 & 1,8 & 1,4 & 1,4 & 0 & 47,02 \% & 22,95 \% & 30,03 \% \\ \text { Implícito II } & 1,8 & 1,126 & 1,4 & 0,726 & 0,674 & 45,02 \% & 31,40 \% & 23,58 \%\end{array}$


Santos $3 \times 0$ Sport

$\begin{array}{lrrrrrrrr}\text { SD 0 } & 1,782 & 1,782 & 1,112 & 1,112 & 0 & 53,06 \% & 23,30 \% & 23,64 \% \\ \text { SD I } & 1,782 & 2,326 & 1,112 & 1,656 & -0,544 & & & \\ \text { "Chance" I } & 1,761 & 1,761 & 1,155 & 1,155 & 0 & 51,57 \% & 23,47 \% & 24,96 \% \\ \text { "Chance" II } & 2,223 & 1,785 & 1,61 & 1,172 & 0,438 & 51,73 \% & 23,27 \% & 24,99 \% \\ \text { Implícito I } & 2 & 2 & 1,4 & 1,4 & 0 & 51,48 \% & 21,66 \% & 26,86 \% \\ \text { Implícito II } & 2 & 1,008 & 1,4 & 0,408 & 0,992 & 49,95 \% & 35,32 \% & 14,73 \%\end{array}$

Santos $2 \times 1$ Corinthians

$\begin{array}{lrrrrrrrr}\text { SD 0 } & 1,865 & 1,865 & 1,054 & 1,054 & 0 & 56,33 \% & 22,52 \% & 21,15 \% \\ \text { SD I } & 1,865 & 2,015 & 1,054 & 1,204 & -0,15 & & & \\ \text { "Chance" I } & 1,842 & 1,842 & 1,086 & 1,086 & 0 & 55,05 \% & 22,76 \% & 22,19 \% \\ \text { "Chance" II } & 2,286 & 1,845 & 1,522 & 1,081 & 0,44 & 55,24 \% & 22,72 \% & 22,04 \% \\ \text { Implícito I } & 1,5 & 1,5 & 1,4 & 1,4 & 0 & 39,86 \% & 24,74 \% & 35,40 \% \\ \text { Implícito II } & 1,5 & 1,259 & 1,4 & 1,159 & 0,241 & 38,67 \% & 27,49 \% & 33,84 \%\end{array}$

Cruzeiro $3 \times 1$ Portuguesa

$\begin{array}{lrrrrrrrr}\text { SD 0 } & 1,812 & 1,812 & 1,193 & 1,193 & 0 & 51,88 \% & 23,05 \% & 25,08 \% \\ \text { SD I } & 1,812 & 1,594 & 1,193 & 0,975 & 0,218 & 51,80 \% & 25,08 \% & 23,12 \% \\ \text { "Chance" I } & 1,706 & 1,706 & 1,205 & 1,205 & 0 & 49,12 \% & 23,88 \% & 27,00 \% \\ \text { "Chance" II } & 2,151 & 1,71 & 1,692 & 1,251 & 0,44 & 48,17 \% & 23,78 \% & 28,04 \% \\ \text { Implícito I } & 1,6 & 1,6 & 1,3 & 1,3 & 0 & 44,44 \% & 24,47 \% & 31,09 \% \\ \text { Implícito II } & 1,6 & 1,226 & 1,3 & 0,926 & 0,374 & 43,05 \% & 29,08 \% & 27,87 \%\end{array}$

Corinthians $2 \times 0$ Santos

$\begin{array}{lrrrrrrrr}\text { SD 0 } & 1,595 & 1,595 & 1,325 & 1,325 & 0 & 43,78 \% & 24,43 \% & 31,79 \% \\ \text { SD I } & 1,595 & 1,795 & 1,325 & 1,525 & -0,2 & & & \\ \text { "Chance" I } & 1,558 & 1,558 & 1,282 & 1,282 & 0 & 43,79 \% & 24,80 \% & 31,40 \% \\ \text { "Chance" II } & 2,014 & 1,574 & 1,793 & 1,353 & 0,44 & 42,67 \% & 24,48 \% & 32,85 \% \\ \text { Implícito I } & 1,7 & 1,7 & 1,6 & 1,6 & 0 & 40,61 \% & 23,00 \% & 36,40 \% \\ \text { Implícito II } & 1,7 & 1,213 & 1,6 & 1,113 & 0,487 & 38,39 \% & 28,13 \% & 33,48 \%\end{array}$

Portuguesa $2 \times 1$ Cruzeiro

$\begin{array}{lrrrrrrrr}\text { SD 0 } & 1,73 & 1,73 & 1,317 & 1,317 & 0 & 47,19 \% & 23,54 \% & 29,27 \% \\ \text { SD I } & 1,73 & 1,584 & 1,317 & 1,171 & 0,146 & 46,92 \% & 24,90 \% & 28,18 \% \\ \text { "Chance" I } & 1,725 & 1,725 & 1,228 & 1,228 & 0 & 49,06 \% & 23,70 \% & 27,23 \% \\ \text { "Chance" II } & 2,179 & 1,738 & 1,706 & 1,266 & 0,44 & 48,52 \% & 23,56 \% & 27,92 \% \\ \text { Implícito I } & 1,5 & 1,5 & 1,4 & 1,4 & 0 & 39,86 \% & 24,74 \% & 35,40 \%\end{array}$

Implícito II $\quad 1,5 \quad 1,126 \quad 1,4 \quad 1,026$

$0,374 \quad 37,80 \% \quad 29,47 \% \quad 32,72 \%$

Corinthians $1 \times 1$ Santos

$\begin{array}{lrrrrrrrr}\text { SD 0 } & 1,611 & 1,611 & 1,273 & 1,273 & 0 & 45,30 \% & 24,46 \% & 30,24 \% \\ \text { SD I } & 1,611 & 1,823 & 1,273 & 1,485 & -0,212 & & & \\ \text { "Chance" I } & 1,578 & 1,578 & 1,239 & 1,239 & 0 & 45,25 \% & 24,79 \% & 29,96 \% \\ \text { "Chance" II } & 2,029 & 1,591 & 1,738 & 1,3 & 0,438 & 44,22 \% & 24,53 \% & 31,25 \% \\ \text { Implícito I } & 2 & 2 & 1,5 & 1,5 & 0 & 49,36 \% & 21,62 \% & 29,03 \% \\ \text { Implícito II } & 2 & 1,159 & 1,5 & 0,659 & 0,841 & 47,83 \% & 31,21 \% & 20,96 \%\end{array}$


Portuguesa 0x1 Cruzeiro

$\begin{array}{lrrrrrrrr}\text { SD 0 } & 1,744 & 1,744 & 1,305 & 1,305 & 0 & 47,78 \% & 23,46 \% & 28,75 \% \\ \text { SD I } & 1,744 & 1,665 & 1,305 & 1,226 & 0,079 & 47,66 \% & 24,16 \% & 28,18 \% \\ \text { "Chance" I } & 1,741 & 1,741 & 1,219 & 1,219 & 0 & 49,64 \% & 23,59 \% & 26,77 \% \\ \text { "Chance" II } & 2,189 & 1,751 & 1,693 & 1,255 & 0,438 & 49,06 \% & 23,48 \% & 27,46 \% \\ \text { Implícito I } & 1,8 & 1,8 & 1,6 & 1,6 & 0 & 42,88 \% & 22,53 \% & 34,58 \% \\ \text { Implícito II } & 1,8 & 1,059 & 1,6 & 0,859 & 0,741 & 39,61 \% & 31,41 \% & 28,98 \%\end{array}$

Cruzeiro $2 \times 2$ Corinthians

$\begin{array}{lrrrrrrrr}\text { SD 0 } & 1,659 & 1,659 & 0,888 & 0,888 & 0 & 55,58 \% & 24,38 \% & 20,04 \% \\ \text { SD I } & 1,659 & 1,712 & 0,888 & 0,941 & -0,053 & & & \\ \text { "Chance" I } & 1,564 & 1,564 & 0,97 & 0,97 & 0 & 51,18 \% & 25,38 \% & 23,44 \% \\ \text { "Chance" II } & 2,029 & 1,593 & 1,363 & 0,927 & 0,436 & 52,97 \% & 25,10 \% & 21,93 \% \\ \text { Implícito I } & 2,4 & 2,4 & 1,4 & 1,4 & 0 & 59,61 \% & 18,99 \% & 21,39 \% \\ \text { Implícito II } & 2,4 & 1,413 & 1,4 & 0,413 & 0,987 & 62,33 \% & 26,96 \% & 10,70 \%\end{array}$

Corinthians 1x1 Cruzeiro

\begin{tabular}{|c|c|c|c|c|c|c|c|c|}
\hline SD 0 & 1,477 & 1,477 & 1,128 & 1,128 & 0 & $45,18 \%$ & $25,89 \%$ & $28,93 \%$ \\
\hline SD I & 1,477 & 1,567 & 1,128 & 1,217 & $-0,09$ & & & \\
\hline "Chance" I & 1,442 & 1,442 & 1,098 & 1,098 & 0 & $44,96 \%$ & $26,28 \%$ & $28,76 \%$ \\
\hline "Chance" II & 1,906 & 1,467 & 1,549 & 1,11 & 0,439 & $45,33 \%$ & $26,03 \%$ & $28,64 \%$ \\
\hline Implícito I & 2 & 2 & 2 & 2 & 0 & $39,65 \%$ & $20,70 \%$ & $39,65 \%$ \\
\hline Implícito II & 2 & 0,996 & 2 & 0,996 & 1,004 & $34,53 \%$ & $30,93 \%$ & $34,53 \%$ \\
\hline Corinthians $2 \times 0$ & Cruzeiro & & & & & & & \\
\hline SD 0 & 1,459 & 1,459 & 1,123 & 1,123 & 0 & $44,83 \%$ & $26,05 \%$ & $29,12 \%$ \\
\hline $\mathrm{SD} I$ & 1,459 & 1,576 & 1,123 & 1,24 & $-0,117$ & & & \\
\hline "Chance" I & 1,425 & 1,425 & 1,095 & 1,095 & 0 & $44,58 \%$ & $26,44 \%$ & $28,98 \%$ \\
\hline "Chance" II & 1,889 & 1,45 & 1,545 & 1,106 & 0,439 & $44,98 \%$ & $26,19 \%$ & $28,83 \%$ \\
\hline Implícito I & 1,6 & 1,6 & 1,6 & 1,6 & 0 & $38,29 \%$ & $23,43 \%$ & $38,29 \%$ \\
\hline Implícito II & 1,6 & 0,926 & 1,6 & 0,926 & 0,674 & $33,84 \%$ & $32,33 \%$ & $33,84 \%$ \\
\hline
\end{tabular}

\section{A.3. Jogos do Torneio Rio-São Paulo de 1999}

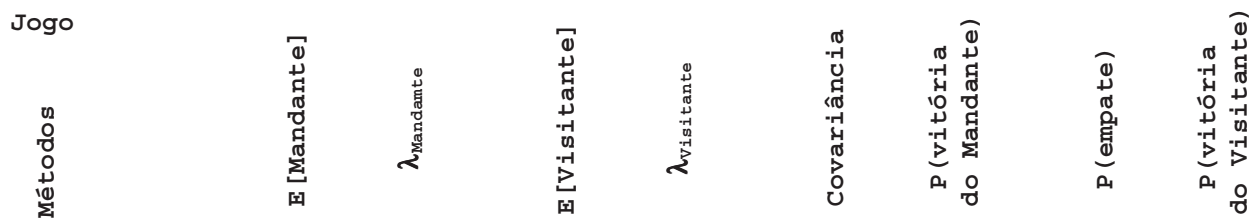

São Paulo 1x0 Flamengo

$\begin{array}{lrrrrrrrr}\text { SD 0 } & 1,588 & 1,588 & 1,264 & 1,264 & 0 & 44,93 \% & 24,65 \% & 30,42 \% \\ \text { SD I } & 1,588 & 1,408 & 1,264 & 1,084 & 0,18 & 44,39 \% & 26,62 \% & 29,00 \% \\ \text { "Chance" I } & 1,477 & 1,477 & 1,259 & 1,259 & 0 & 42,27 \% & 25,45 \% & 32,28 \% \\ \text { "Chance" II } & 2,616 & 1,489 & 2,404 & 1,277 & 1,127 & 42,16 \% & 25,30 \% & 32,52 \% \\ \text { Implícito I } & 2 & 2 & 1,7 & 1,7 & 0 & 45,29 \% & 21,38 \% & 33,33 \% \\ \text { Implícito II } & 2 & 1,296 & 1,7 & 0,996 & 0,704 & 43,38 \% & 28,02 \% & 28,60 \%\end{array}$


Fluminense $2 \times 0$ Santos

$\begin{array}{lrrrrrrrr}\text { SD 0 } & 1,473 & 1,473 & 1,617 & 1,617 & 0 & 34,96 \% & 23,83 \% & 41,21 \% \\ \text { SD I } & 1,473 & 1,622 & 1,617 & 1,766 & -0,149 & & & \\ \text { "Chance" I } & 1,403 & 1,403 & 1,642 & 1,642 & 0 & 32,83 \% & 23,91 \% & 43,26 \% \\ \text { "Chance" II } & 2,534 & 1,406 & 2,803 & 1,676 & 1,127 & 32,31 \% & 23,70 \% & 43,97 \% \\ \text { Implícito I } & 1,4 & 1,4 & 1,8 & 1,8 & 0 & 30,03 \% & 22,95 \% & 47,02 \% \\ \text { Implícito II } & 1,4 & 1,013 & 1,8 & 1,413 & 0,387 & 27,01 \% & 26,80 \% & 46,19 \%\end{array}$

Botafogo $6 \times 1$ Corinthians

$\begin{array}{lrrrrrrrr}\text { SD 0 } & 1,654 & 1,654 & 0,626 & 0,626 & 0 & 62,34 \% & 23,90 \% & 13,76 \% \\ \text { SD I } & 1,654 & 2,073 & 0,626 & 1,045 & -0,419 & & & \\ \text { "Chance" I } & 1,548 & 1,548 & 0,784 & 0,784 & 0 & 55,47 \% & 25,59 \% & 18,94 \% \\ \text { "Chance" II } & 2,672 & 1,545 & 1,797 & 0,67 & 1,127 & 58,43 \% & 25,50 \% & 16,06 \% \\ \text { Implícito I } & 1,1 & 1,1 & 2 & 2 & 0 & 20,39 \% & 21,40 \% & 58,21 \% \\ \text { Implícito II } & 1,1 & 0,813 & 2 & 1,713 & 0,287 & 17,55 \% & 23,67 \% & 58,78 \%\end{array}$

Palmeiras $1 \times 5$ Vasco

$\begin{array}{lrrrrrrrr}\text { SD 0 } & 1,678 & 1,678 & 1,167 & 1,167 & 0 & 49,31 \% & 24,14 \% & 26,54 \% \\ \text { SD I } & 1,678 & 1,692 & 1,167 & 1,181 & -0,014 & & & \\ \text { "Chance" I } & 1,563 & 1,563 & 1,228 & 1,228 & 0 & 45,10 \% & 24,93 \% & 29,97 \% \\ \text { "Chance" II } & 2,713 & 1,585 & 2,365 & 1,237 & 1,127 & 45,44 \% & 24,74 \% & 29,80 \% \\ \text { Implícito I } & 1,9 & 1,9 & 1,4 & 1,4 & 0 & 49,28 \% & 22,31 \% & 28,41 \% \\ \text { Implícito II } & 1,9 & 1,126 & 1,4 & 0,626 & 0,774 & 47,68 \% & 31,90 \% & 20,41 \%\end{array}$

Corinthians 1x2 São Paulo

$\begin{array}{lrrrrrrrr}\text { SD 0 } & 1,188 & 1,188 & 1,606 & 1,606 & 0 & 28,23 \% & 24,69 \% & 47,08 \% \\ \text { SD I } & 1,188 & 1,644 & 1,606 & 2,062 & -0,456 & & & \\ \text { "Chance" I } & 1,269 & 1,269 & 1,649 & 1,649 & 0 & 29,49 \% & 24,20 \% & 46,31 \% \\ \text { "Chance" II } & 2,553 & 1,351 & 2,839 & 1,637 & 1,202 & 31,67 \% & 24,09 \% & 44,21 \% \\ \text { Implícito I } & 1,5 & 1,5 & 1,6 & 1,6 & 0 & 35,92 \% & 23,82 \% & 40,26 \% \\ \text { Implícito II } & 1,5 & 1,059 & 1,6 & 1,159 & 0,441 & 33,02 \% & 28,94 \% & 38,04 \%\end{array}$

Flamengo 4x4 Botafogo

$\begin{array}{lrrrrrrrr}\text { SD 0 } & 1,032 & 1,032 & 1,455 & 1,455 & 0 & 26,80 \% & 26,35 \% & 46,85 \% \\ \text { SD I } & 1,032 & 0,75 & 1,455 & 1,173 & 0,282 & 23,54 \% & 30,60 \% & 45,86 \% \\ \text { "Chance" I } & 1,212 & 1,212 & 1,408 & 1,408 & 0 & 32,37 \% & 26,12 \% & 41,51 \% \\ \text { "Chance" II } & 2,406 & 1,203 & 2,623 & 1,42 & 1,202 & 31,90 \% & 26,07 \% & 42,01 \% \\ \text { Implícito I } & 1,7 & 1,7 & 1,7 & 1,7 & 0 & 38,68 \% & 22,64 \% & 38,68 \% \\ \text { Implícito II } & 1,7 & 1,113 & 1,7 & 1,113 & 0,587 & 35,54 \% & 28,92 \% & 35,54 \%\end{array}$

Vasco 2x4 Fluminense

\begin{tabular}{|c|c|c|c|c|c|c|c|c|}
\hline SD 0 & 1,921 & 1,921 & 0,981 & 0,981 & 0 & $59,33 \%$ & $21,87 \%$ & $18,81 \%$ \\
\hline SD I & 1,921 & 1,358 & 0,981 & 0,418 & 0,563 & $60,67 \%$ & $27,99 \%$ & $11,34 \%$ \\
\hline "Chance" I & 1,874 & 1,874 & 1,254 & 1,254 & 0 & $51,89 \%$ & $22,56 \%$ & $25,55 \%$ \\
\hline "Chance" II & 3,052 & 1,85 & 2,467 & 1,265 & 1,202 & $51,09 \%$ & $22,74 \%$ & $26,14 \%$ \\
\hline Implícito I & 2 & 2 & 1,4 & 1,4 & 0 & $51,48 \%$ & $21,66 \%$ & $26,86 \%$ \\
\hline Implícito II & 2 & 1,819 & 1,4 & 1,219 & 0,181 & $51,45 \%$ & $22,99 \%$ & $25,57 \%$ \\
\hline
\end{tabular}


Santos 3x1 Palmeiras

$\begin{array}{lrrrrrrrr}\text { SD 0 } & 2,002 & 2,002 & 1,121 & 1,121 & 0 & 57,77 \% & 21,42 \% & 20,81 \% \\ \text { SD I } & 2,002 & 3,198 & 1,121 & 2,317 & -1,195 & & & \\ \text { "Chance" I } & 1,764 & 1,764 & 1,374 & 1,374 & 0 & 46,77 \% & 23,22 \% & 30,02 \% \\ \text { "Chance" II } & 2,946 & 1,743 & 2,604 & 1,402 & 1,202 & 45,65 \% & 23,30 \% & 31,02 \% \\ \text { Implícito I } & 2,3 & 2,3 & 1,1 & 1,1 & 0 & 64,25 \% & 18,89 \% & 16,86 \% \\ \text { Implícito II } & 2,3 & 2,119 & 1,1 & 0,919 & 0,181 & 64,94 \% & 19,78 \% & 15,27 \%\end{array}$

Fluminense 4x0 Palmeiras

$\begin{array}{lrrrrrrrr}\text { SD 0 } & 1,793 & 1,793 & 1,359 & 1,359 & 0 & 47,74 \% & 23,05 \% & 29,20 \% \\ \text { SD I } & 1,793 & 2,189 & 1,359 & 1,755 & -0,396 & & & \\ \text { "Chance" I } & 1,737 & 1,737 & 1,351 & 1,351 & 0 & 46,61 \% & 23,44 \% & 29,96 \% \\ \text { "Chance" II } & 3,138 & 1,731 & 2,79 & 1,383 & 1,407 & 45,73 \% & 23,42 \% & 30,79 \% \\ \text { Implícito I } & 1,5 & 1,5 & 1 & 1 & 0 & 48,79 \% & 25,98 \% & 25,22 \% \\ \text { Implícito II } & 1,5 & 1,813 & 1 & 1,313 & -0,313 & & & \end{array}$

Santos 0x0 Vasco

$\begin{array}{lrrrrrrrr}\text { SD 0 } & 1,873 & 1,873 & 1,207 & 1,207 & 0 & 52,95 \% & 22,56 \% & 24,49 \% \\ \text { SD I } & 1,873 & 2,019 & 1,207 & 1,353 & -0,146 & & & \\ \text { "Chance" I } & 1,862 & 1,862 & 1,254 & 1,254 & 0 & 51,63 \% & 22,65 \% & 25,72 \% \\ \text { "Chance" II } & 3,25 & 1,843 & 2,697 & 1,29 & 1,407 & 50,34 \% & 22,77 \% & 26,82 \% \\ \text { Implícito I } & 2 & 2 & 1,6 & 1,6 & 0 & 47,29 \% & 21,53 \% & 31,18 \% \\ \text { Implícito II } & 2 & 1,619 & 1,6 & 1,219 & 0,381 & 46,69 \% & 24,53 \% & 28,78 \%\end{array}$

São Paulo 2x0 Botafogo

$\begin{array}{lrrrrrrrr}\text { SD 0 } & 1,562 & 1,562 & 1,551 & 1,551 & 0 & 38,34 \% & 23,79 \% & 37,86 \% \\ \text { SD I } & 1,562 & 1,733 & 1,551 & 1,722 & -0,171 & & & \\ \text { "Chance" I } & 1,508 & 1,508 & 1,543 & 1,543 & 0 & 37,20 \% & 24,07 \% & 38,73 \% \\ \text { "Chance" II } & 2,926 & 1,519 & 2,991 & 1,584 & 1,407 & 36,67 \% & 23,82 \% & 39,45 \% \\ \text { Implícito I } & 2 & 2 & 1,7 & 1,7 & 0 & 45,29 \% & 21,38 \% & 33,33 \% \\ \text { Implícito II } & 2 & 1,519 & 1,7 & 1,219 & 0,481 & 44,20 \% & 25,28 \% & 30,51 \%\end{array}$

Flamengo 2xo Corinthians

$\begin{array}{lrrrrrrrr}\text { SD 0 } & 1,837 & 1,837 & 0,694 & 0,694 & 0 & 64,70 \% & 21,83 \% & 13,47 \% \\ \text { SD I } & 1,837 & 0,424 & 0,694 & -0,719 & 1,413 & & & \\ \text { "Chance" I } & 1,741 & 1,741 & 0,816 & 0,816 & 0 & 59,35 \% & 23,36 \% & 17,29 \% \\ \text { "Chance" II } & 3,158 & 1,751 & 2,115 & 0,708 & 1,407 & 62,37 \% & 22,92 \% & 14,66 \% \\ \text { Implícito I } & 2 & 2 & 1,6 & 1,6 & 0 & 47,29 \% & 21,53 \% & 31,18 \% \\ \text { Implícito II } & 2 & 1,396 & 1,6 & 0,996 & 0,604 & 46,13 \% & 27,03 \% & 26,84 \%\end{array}$

Flamengo 0x1 São Paulo

$\begin{array}{lrrrrrrrr}\text { SD 0 } & 1,729 & 1,729 & 0,926 & 0,926 & 0 & 56,31 \% & 23,68 \% & 20,02 \% \\ \text { SD I } & 1,729 & 1,346 & 0,926 & 0,544 & 0,382 & 56,65 \% & 28,36 \% & 14,99 \% \\ \text { "Chance" I } & 1,627 & 1,627 & 1,014 & 1,014 & 0 & 51,67 \% & 24,73 \% & 23,60 \% \\ \text { "Chance" II } & 2,737 & 1,613 & 2,102 & 0,977 & 1,125 & 52,21 \% & 24,89 \% & 22,89 \% \\ \text { Implícito I } & 1,9 & 1,9 & 2 & 2 & 0 & 37,57 \% & 20,97 \% & 41,47 \% \\ \text { Implícito II } & 1,9 & 0,526 & 2 & 0,626 & 1,374 & 25,33 \% & 42,91 \% & 31,76 \%\end{array}$


Santos 4x1 Fluminense

$\begin{array}{lrrrrrrrr}\text { SD 0 } & 1,969 & 1,969 & 0,999 & 0,999 & 0 & 59,93 \% & 21,46 \% & 18,61 \% \\ \text { SD I } & 1,969 & 2,31 & 0,999 & 1,34 & -0,341 & & & \\ \text { "Chance" I } & 1,913 & 1,913 & 1,073 & 1,073 & 0 & 56,95 \% & 22,11 \% & 20,93 \% \\ \text { "Chance" II } & 3,007 & 1,882 & 2,203 & 1,079 & 1,125 & 56,10 \% & 22,40 \% & 21,47 \% \\ \text { Implícito I } & 2 & 2 & 1 & 1 & 0 & 60,57 \% & 21,17 \% & 18,26 \% \\ \text { Implícito II } & 2 & 1,996 & 1 & 0,996 & 0,004 & 60,58 \% & 21,20 \% & 18,22 \%\end{array}$

Palmeiras 2x1 Fluminense

$\begin{array}{lrrrrrrrr}\text { SD 0 } & 1,956 & 1,956 & 1,587 & 1,587 & 0 & 46,60 \% & 21,77 \% & 31,62 \% \\ \text { SD I } & 1,956 & 2,881 & 1,587 & 2,512 & -0,925 & & & \\ \text { "Chance" I } & 1,707 & 1,707 & 1,546 & 1,546 & 0 & 41,85 \% & 23,14 \% & 35,02 \% \\ \text { "Chance" II } & 2,823 & 1,707 & 2,725 & 1,61 & 1,115 & 40,57 \% & 22,93 \% & 36,47 \% \\ \text { Implícito I } & 2 & 2 & 2 & 2 & 0 & 39,65 \% & 20,70 \% & 39,65 \% \\ \text { Implícito II } & 2 & 0,813 & 2 & 0,813 & 1,187 & 32,51 \% & 34,98 \% & 32,51 \%\end{array}$

Vasco $3 \times 2$ Santos

$\begin{array}{lrrrrrrrr}\text { SD 0 } & 1,592 & 1,592 & 1,175 & 1,175 & 0 & 47,03 \% & 24,83 \% & 28,15 \% \\ \text { SD I } & 1,592 & 1,086 & 1,175 & 0,669 & 0,506 & 45,24 \% & 32,33 \% & 22,43 \% \\ \text { "Chance" I } & 1,54 & 1,54 & 1,236 & 1,236 & 0 & 44,38 \% & 25,07 \% & 30,55 \% \\ \text { "Chance" II } & 2,706 & 1,591 & 2,394 & 1,279 & 1,115 & 44,66 \% & 24,59 \% & 30,74 \% \\ \text { Implícito I } & 2,2 & 2,2 & 1,8 & 1,8 & 0 & 47,50 \% & 20,35 \% & 32,15 \% \\ \text { Implícito II } & 2,2 & 1,196 & 1,8 & 0,796 & 1,004 & 45,37 \% & 30,07 \% & 24,55 \%\end{array}$

Corinthians 0x3 Flamengo

$\begin{array}{lrrrrrrrr}\text { SD 0 } & 1,166 & 1,166 & 1,227 & 1,227 & 0 & 34,67 \% & 27,69 \% & 37,63 \% \\ \text { SD I } & 1,166 & 0,173 & 1,227 & 0,234 & 0,993 & 12,85 \% & 69,23 \% & 17,92 \% \\ \text { "Chance" I } & 1,108 & 1,108 & 1,236 & 1,236 & 0 & 32,89 \% & 27,98 \% & 39,14 \% \\ \text { "Chance" II } & 2,312 & 1,197 & 2,401 & 1,285 & 1,115 & 34,34 \% & 27,08 \% & 38,57 \% \\ \text { Implícito I } & 1,4 & 1,4 & 1,4 & 1,4 & 0 & 37,36 \% & 25,28 \% & 37,36 \% \\ \text { Implícito II } & 1,4 & 1,259 & 1,4 & 1,259 & 0,141 & 36,56 \% & 26,89 \% & 36,56 \%\end{array}$

Botafogo 2x1 São Paulo

$\begin{array}{lrrrrrrrr}\text { SD 0 } & 1,899 & 1,899 & 1,044 & 1,044 & 0 & 57,33 \% & 22,19 \% & 20,48 \% \\ \text { SD I } & 1,899 & 2,106 & 1,044 & 1,251 & -0,207 & & & \\ \text { "Chance" I } & 1,827 & 1,827 & 1,108 & 1,108 & 0 & 54,20 \% & 22,90 \% & 22,90 \% \\ \text { "Chance" II } & 2,923 & 1,808 & 2,201 & 1,085 & 1,115 & 54,27 \% & 23,06 \% & 22,65 \% \\ \text { Implícito I } & 1 & 1 & 1,4 & 1,4 & 0 & 26,88 \% & 26,97 \% & 46,15 \% \\ \text { Implícito II } & 1 & 1,413 & 1,4 & 1,813 & -0,413 & & & \end{array}$

São Paulo 1x1 Corinthians

$\begin{array}{lrrrrrrrr}\text { SD 0 } & 1,63 & 1,63 & 1,134 & 1,134 & 0 & 48,91 \% & 24,58 \% & 26,51 \% \\ \text { SD I } & 1,63 & 2,323 & 1,134 & 1,827 & -0,693 & & & \\ \text { "Chance" I } & 1,879 & 1,879 & 0,996 & 0,996 & 0 & 58,03 \% & 22,30 \% & 19,67 \% \\ \text { "Chance" II } & 3,148 & 1,889 & 2,297 & 1,039 & 1,258 & 57,20 \% & 22,27 \% & 20,48 \% \\ \text { Implícito I } & 1,5 & 1,5 & 0,9 & 0,9 & 0 & 51,26 \% & 26,13 \% & 22,61 \% \\ \text { Implícito II } & 1,5 & 1,508 & 0,9 & 0,908 & -0,008 & & & \end{array}$


Botafogo 1x1 Flamengo

$\begin{array}{lrrrrrrrr}\text { SD 0 } & 1,761 & 1,761 & 1,37 & 1,37 & 0 & 46,76 \% & 23,25 \% & 29,99 \% \\ \text { SD I } & 1,761 & 0,299 & 1,37 & -0,093 & 1,463 & & & \\ \text { "Chance" I } & 1,807 & 1,807 & 1,594 & 1,594 & 0 & 43,15 \% & 22,51 \% & 34,34 \% \\ \text { "Chance" II } & 3,085 & 1,826 & 2,868 & 1,61 & 1,258 & 43,25 \% & 22,38 \% & 34,31 \% \\ \text { Implícito I } & 1,5 & 1,5 & 2 & 2 & 0 & 29,03 \% & 21,62 \% & 49,36 \% \\ \text { Implícito II } & 1,5 & 1,219 & 2 & 1,719 & 0,281 & 27,13 \% & 23,76 \% & 49,11 \%\end{array}$

Corinthians $3 \times 2$ Botafogo

$\begin{array}{lrrrrrrrr}\text { SD 0 } & 1,439 & 1,439 & 1,436 & 1,436 & 0 & 37,62 \% & 24,90 \% & 37,48 \% \\ \text { SD I } & 1,439 & 1,29 & 1,436 & 1,287 & 0,149 & 36,81 \% & 26,52 \% & 36,67 \% \\ \text { "Chance" I } & 1,022 & 1,022 & 1,594 & 1,594 & 0 & 24,31 \% & 25,04 \% & 50,66 \% \\ \text { "Chance" II } & 2,378 & 1,092 & 2,952 & 1,666 & 1,286 & 24,91 \% & 24,32 \% & 50,74 \% \\ \text { Implícito I } & 1,2 & 1,2 & 1,4 & 1,4 & 0 & 32,21 \% & 26,23 \% & 41,56 \% \\ \text { Implícito II } & 1,2 & 1,259 & 1,4 & 1,459 & -0,059 & & & \end{array}$

Vasco 2x0 Palmeiras

\begin{tabular}{|c|c|c|c|c|c|c|c|c|}
\hline $\mathrm{SD} 0$ & 2,285 & 2,285 & 0,933 & 0,933 & 0 & $67,77 \%$ & $18,32 \%$ & $13,90 \%$ \\
\hline SD I & 2,285 & 2,234 & 0,933 & 0,882 & 0,051 & $68,02 \%$ & $18,54 \%$ & $13,44 \%$ \\
\hline "Chance" I & 2,294 & 2,294 & 1,089 & 1,089 & 0 & $64,37 \%$ & $18,90 \%$ & $16,73 \%$ \\
\hline "Chance" II & 3,458 & 2,172 & 2,348 & 1,062 & 1,286 & $62,54 \%$ & $19,83 \%$ & $17,54 \%$ \\
\hline Implícito I & 2,1 & 2,1 & 1,4 & 1,4 & 0 & $53,61 \%$ & $21,00 \%$ & $25,39 \%$ \\
\hline Implícito II & 2,1 & 1,596 & 1,4 & 0,896 & 0,504 & $53,82 \%$ & $25,08 \%$ & $21,11 \%$ \\
\hline Palmeiras $3 \times 2$ & Santos & & & & & & & \\
\hline $\mathrm{SD} 0$ & 1,518 & 1,518 & 1,594 & 1,594 & 0 & $36,47 \%$ & $23,78 \%$ & $39,75 \%$ \\
\hline SD I & 1,518 & 2,663 & 1,594 & 2,739 & $-1,145$ & & & \\
\hline "Chance" I & 1,48 & 1,48 & 1,763 & 1,763 & 0 & $32,50 \%$ & $23,02 \%$ & $44,48 \%$ \\
\hline "Chance" II & 1,103 & 1,53 & 1,384 & 1,81 & $-0,427$ & & & \\
\hline Implícito I & 1,6 & 1,6 & 1,8 & 1,8 & 0 & $34,58 \%$ & $22,53 \%$ & $42,88 \%$ \\
\hline Implícito II & 1,6 & 0,859 & 1,8 & 1,059 & 0,741 & $28,98 \%$ & $31,41 \%$ & $39,61 \%$ \\
\hline
\end{tabular}

Vasco $2 \times 3$ São Paulo

\begin{tabular}{|c|c|c|c|c|c|c|c|c|}
\hline SD 0 & 1,954 & 1,954 & 0,75 & 0,75 & 0 & $65,75 \%$ & $20,74 \%$ & $13,51 \%$ \\
\hline SD I & 1,954 & 1,71 & 0,75 & 0,506 & 0,244 & $66,96 \%$ & $22,58 \%$ & $10,46 \%$ \\
\hline "Chance" I & 1,818 & 1,818 & 0,966 & 0,966 & 0 & $57,39 \%$ & $22,84 \%$ & $19,78 \%$ \\
\hline "Chance" II & 1,273 & 1,781 & 0,421 & 0,929 & $-0,508$ & & & \\
\hline Implícito I & 1,6 & 1,6 & 1,4 & 1,4 & 0 & $42,31 \%$ & $24,17 \%$ & $33,52 \%$ \\
\hline Implícito II & 1,6 & 1,259 & 1,4 & 1,059 & 0,341 & $40,89 \%$ & $28,05 \%$ & $31,06 \%$ \\
\hline
\end{tabular}

Santos 1x0 Botafogo

\begin{tabular}{|c|c|c|c|c|c|c|c|c|}
\hline SD 0 & 2,192 & 2,192 & 1,08 & 1,08 & 0 & $62,62 \%$ & $19,71 \%$ & $17,67 \%$ \\
\hline SD I & 2,192 & 2,261 & 1,08 & 1,148 & $-0,068$ & & & \\
\hline "Chance" I & 1,995 & 1,995 & 1,266 & 1,266 & 0 & $54,32 \%$ & $21,65 \%$ & $24,03 \%$ \\
\hline "Chance" II & 1,477 & 1,985 & 0,788 & 1,297 & $-0,508$ & & & \\
\hline Implícito I & 2 & 2 & 1,5 & 1,5 & 0 & $49,36 \%$ & $21,62 \%$ & $29,03 \%$ \\
\hline Implícito II & 2 & 1,496 & 1,5 & 0,996 & 0,504 & $48,79 \%$ & $26,03 \%$ & $25,18 \%$ \\
\hline
\end{tabular}


Botafogo 0x2 Santos

$\begin{array}{lrrrrrrrr}\text { SD 0 } & 1,656 & 1,656 & 1,272 & 1,272 & 0 & 46,42 \% & 24,14 \% & 29,44 \% \\ \text { SD I } & 1,656 & 1,474 & 1,272 & 1,09 & 0,182 & 45,98 \% & 26,02 \% & 28,00 \% \\ \text { "Chance" I } & 1,628 & 1,628 & 1,356 & 1,356 & 0 & 43,91 \% & 24,13 \% & 31,96 \% \\ \text { "Chance" II } & 1,16 & 1,643 & 0,94 & 1,422 & -0,482 & & & \\ \text { Implícito I } & 1,4 & 1,4 & 1,5 & 1,5 & 0 & 35,40 \% & 24,74 \% & 39,86 \% \\ \text { Implícito II } & 1,4 & 1,313 & 1,5 & 1,413 & 0,087 & 34,89 \% & 25,63 \% & 39,48 \%\end{array}$

São Paulo 1×3 Vasco

$\begin{array}{lrrrrrrrr}\text { SD 0 } & 1,699 & 1,699 & 1,251 & 1,251 & 0 & 47,91 \% & 23,87 \% & 28,22 \% \\ \text { SD I } & 1,699 & 1,494 & 1,251 & 1,046 & 0,205 & 47,54 \% & 25,95 \% & 26,51 \% \\ \text { "Chance" I } & 1,584 & 1,584 & 1,335 & 1,335 & 0 & 43,28 \% & 24,47 \% & 32,25 \% \\ \text { "Chance" II } & 1,148 & 1,631 & 0,868 & 1,35 & -0,482 & & & \\ \text { Implícito I } & 2 & 2 & 1,4 & 1,4 & 0 & 51,48 \% & 21,66 \% & 26,86 \% \\ \text { Implícito II } & 2 & 1,819 & 1,4 & 1,219 & 0,181 & 51,45 \% & 22,99 \% & 25,57 \%\end{array}$

Vasco $3 \times 1$ Santos

$\begin{array}{lrrrrrrrr}\text { SD 0 } & 1,577 & 1,577 & 1,091 & 1,091 & 0 & 48,59 \% & 25,11 \% & 26,30 \% \\ \text { SD I } & 1,577 & 0,838 & 1,091 & 0,352 & 0,738 & 45,22 \% & 40,06 \% & 14,72 \% \\ \text { "Chance" I } & 1,753 & 1,753 & 1,421 & 1,421 & 0 & 45,49 \% & 23,20 \% & 31,30 \% \\ \text { "Chance" II } & 1,33 & 1,786 & 1,042 & 1,499 & -0,457 & & & \\ \text { Implícito I } & 2 & 2 & 2 & 2 & 0 & 39,65 \% & 20,70 \% & 39,65 \% \\ \text { Implícito II } & 2 & 0,996 & 2 & 0,996 & 1,004 & 34,53 \% & 30,93 \% & 34,53 \%\end{array}$

Santos 1x2 Vasco

\begin{tabular}{|c|c|c|c|c|c|c|c|c|}
\hline SD 0 & 1,572 & 1,572 & 1,515 & 1,515 & 0 & $39,29 \%$ & $23,90 \%$ & $36,81 \%$ \\
\hline $\mathrm{SD} I$ & 1,572 & 1,076 & 1,515 & 1,019 & 0,496 & $36,47 \%$ & $29,98 \%$ & $33,54 \%$ \\
\hline "Chance" I & 1,91 & 1,91 & 1,92 & 1,92 & 0 & $39,21 \%$ & $21,20 \%$ & $39,59 \%$ \\
\hline "Chance" II & 1,494 & 1,913 & 1,502 & 1,921 & $-0,419$ & & & \\
\hline Implícito I & 2 & 2 & 1,4 & 1,4 & 0 & $51,48 \%$ & $21,66 \%$ & $26,86 \%$ \\
\hline Implícito II & 2 & 1,596 & 1,4 & 0,996 & 0,404 & $51,34 \%$ & $25,04 \%$ & $23,61 \%$ \\
\hline
\end{tabular}




\section{Apêndice B \\ Curvas de Calibração}

As curvas de calibração apresentadas neste apêndice são os gráficos a partir dos quais se calculou a Medida de Confiabilidade (definidas no Capítulo 5) para cada um dos métodos (ver Capítulo 6).

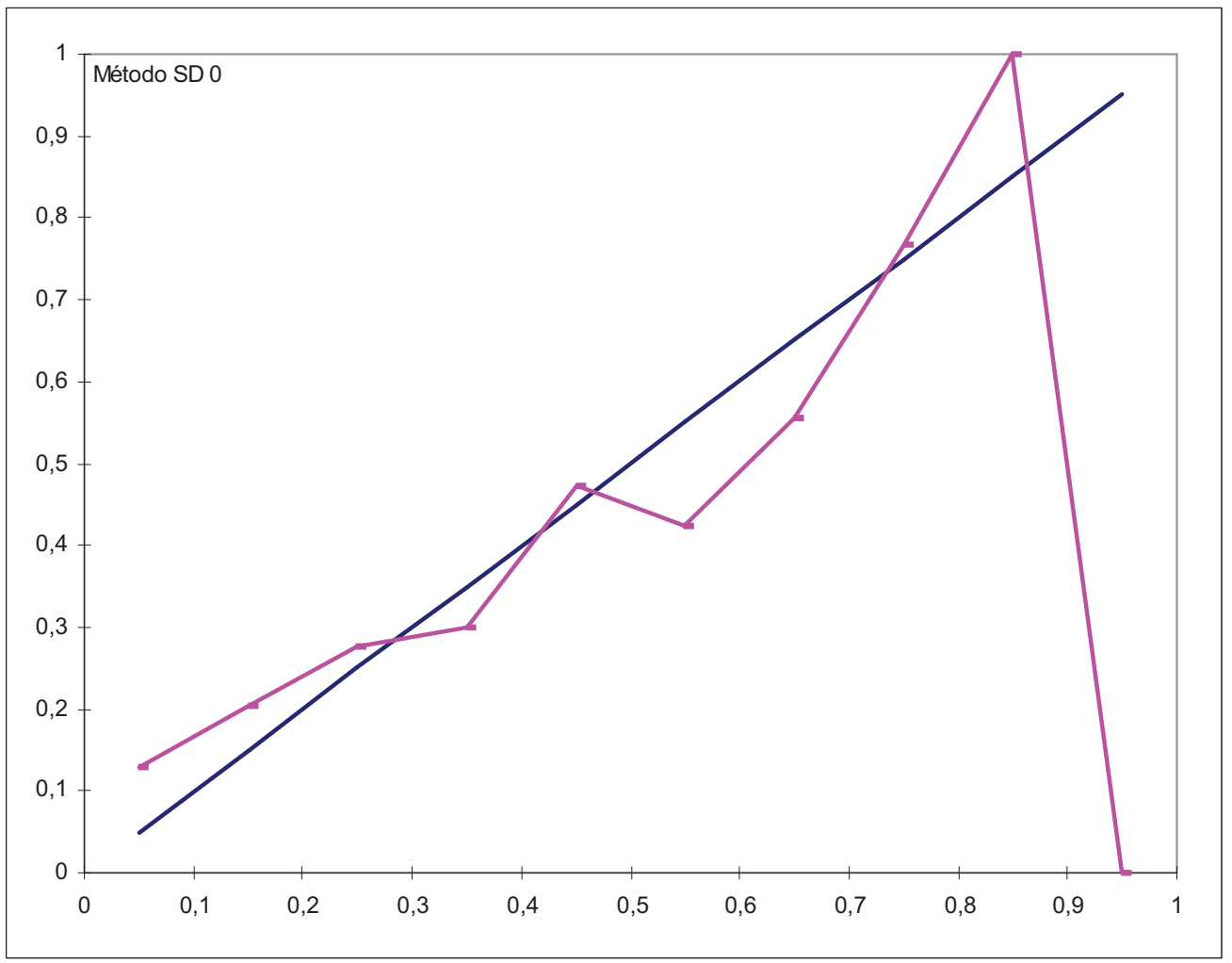


120
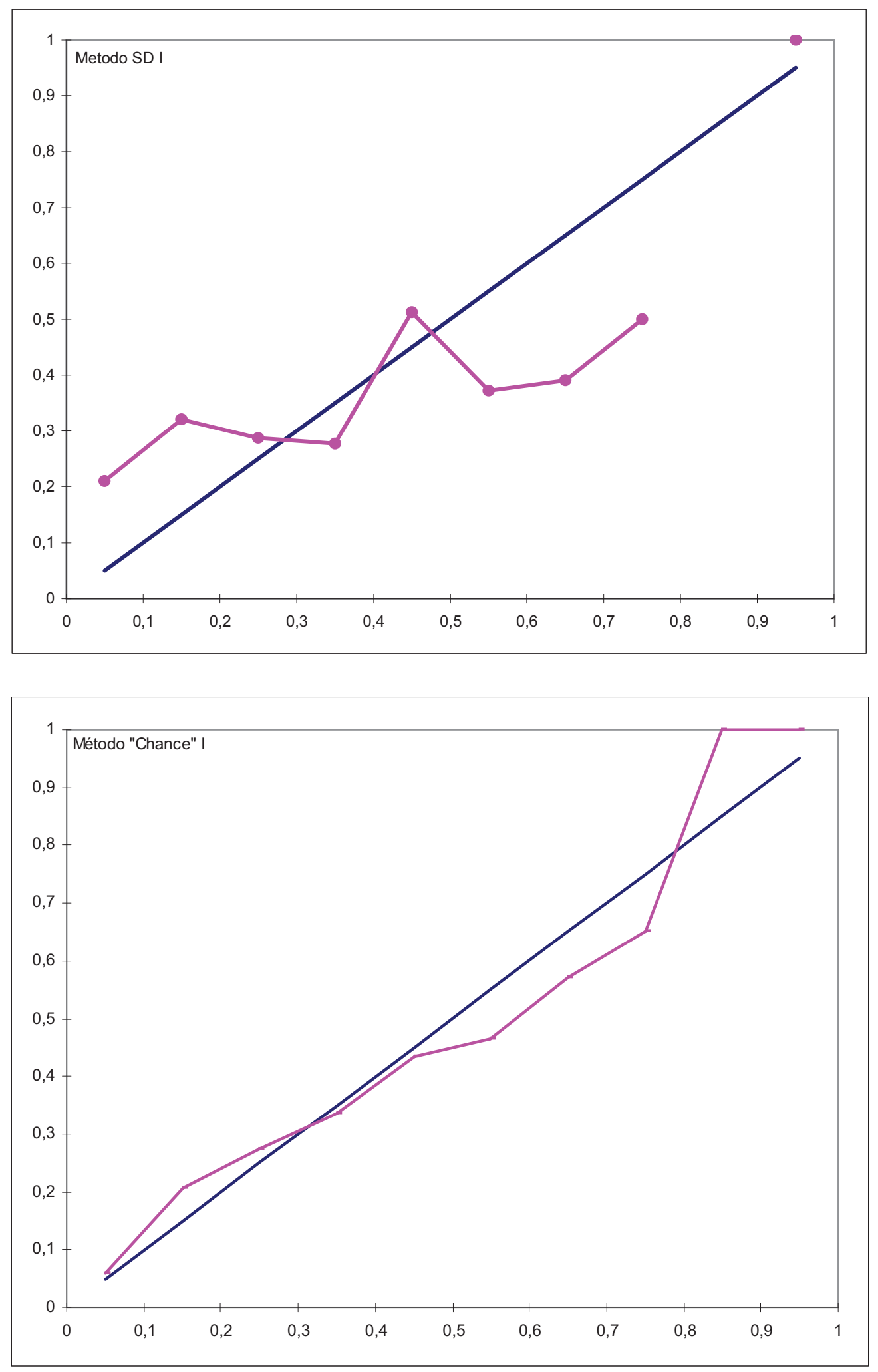

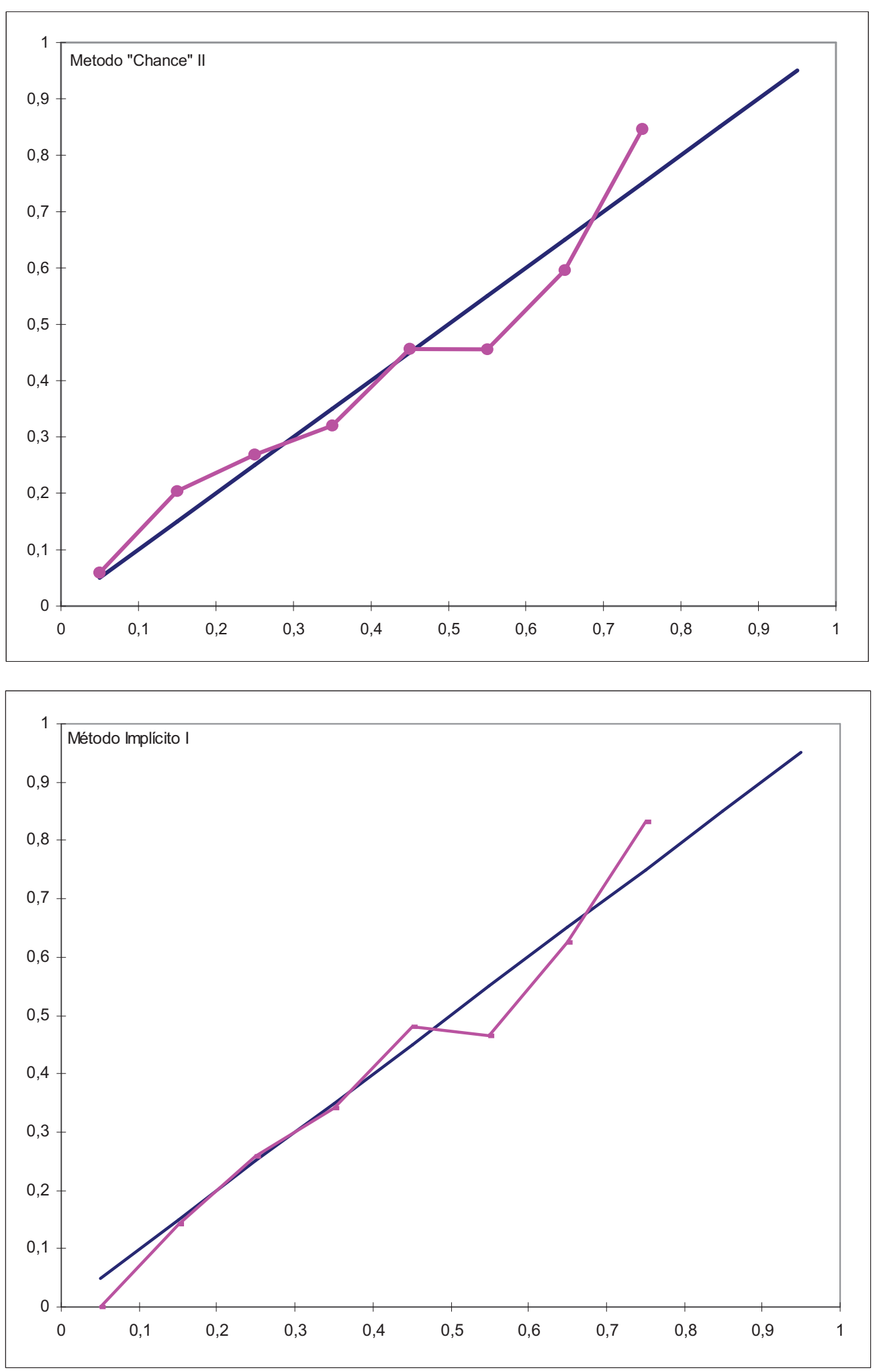
122
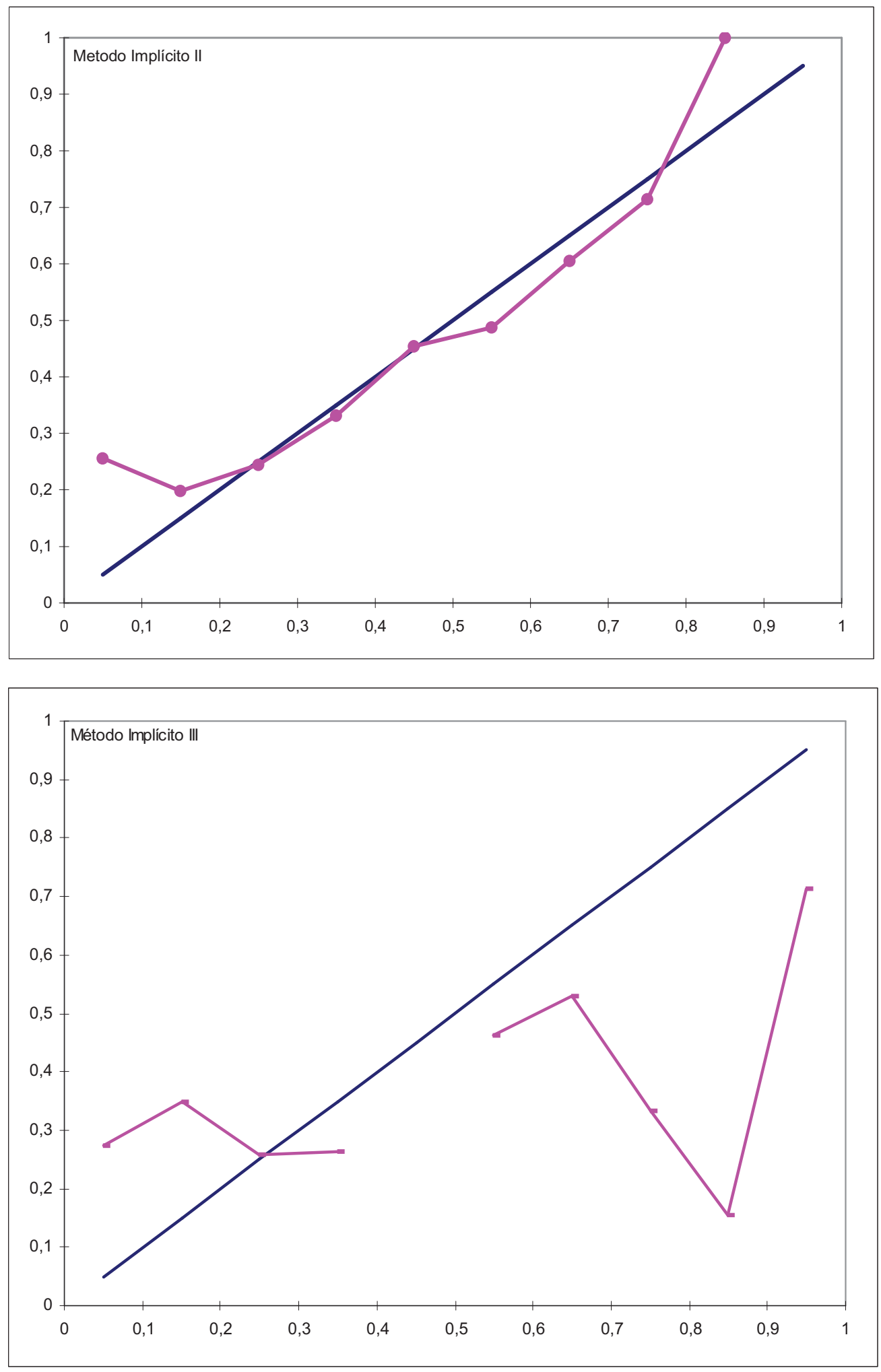


\title{
Apêndice $C$
}

Texto sobre o Site de Previsões para o Campeonato Brasileiro 1999

\author{
PREVISÕES PROBABILÍSTICAS APLICADAS AO \\ CAMPEONATO BRASILEIRO DE FUTEBOL
}

\author{
http://www.ime.usp.br/ mlarruda/bras99.html
}

\author{
Marcelo Leme de Arruda \\ Sergio Wechsler \\ IME - USP
}

Este site mantém um acompanhamento quantitativo do campeonato brasileiro de futebol de 1999. Trata-se de uma aplicação de um modelo teórico geral de previsões probabilísticas, desenvolvido por pesquisadores do Instituto de Matemática e Estatística da USP, com auxílio parcial da Fapesp.

A inovação nesta abordagem reside na obtenção de probabilidades para diversos eventos de interesse para o público, tais como classificação para fases posteriores da competição, rebaixamento a divisões inferiores, resultados de cada partida ainda a ser jogada, além de avaliações de número de pontos necessários para garantir probabilisticamente a realização de eventos como classificação ou permanência na mesma divisão. Todas as previsões são atualizadas após cada rodada de jogos. Tudo isto está em oposição ao que a imprensa especializada apresenta ao público como "Estatística", consistindo em mera tabulação de dados referentes a partidas já realizadas, sem qualquer avaliação futura

A metodologia desenvolvida faz uso de uma base de dados extremamente grande composta de resultados de diversas competições anteriores, além da corrente. Um modelo probabilístico foi construído e técnicas de estimação estatística foram especialmente desenvolvidas.

A qualidade das previsões foi testada em competições do ano passado. $O$ trabalho de pesquisa inclui um aperfeiçoamento das técnicas de cotejo de probabilidades anunciadas com freqüências de realizações posteriores. Estas técnicas possuem destaque na literatura científica, sendo muito aplicadas a aferições de previsões meteorológicas, por exemplo.

Este site está atingindo um público leigo muito grande. Além de considerar-se o papel do futebol no lazer da sociedade brasileira, deve ser ressaltada a contribuição educativa que se alcança, uma vez que cada vez mais o cidadão necessita de uma compreensão mínima da linguagem e postura probabilísticas, essencial em todas as realizações científicas que o atingem e o beneficiam. Essa necessidade está presente na leitura de previsões meteorológicas, diagnósticos clínicos, previsões econômicas, previsões eleitorais e tantas outras. 